Florida International University FIU Digital Commons

FIU Electronic Theses and Dissertations

University Graduate School

11-10-2015

\title{
Organometallic Rhenium Dyes for Nitric Oxide Detection and Imaging
}

Lissette I. Lozano-Lewis

Florida International University, 1loza009@fiu.edu

DOI: $10.25148 /$ etd.FIDC000203

Follow this and additional works at: https://digitalcommons.fiu.edu/etd

Part of the Analytical Chemistry Commons, Inorganic Chemistry Commons, and the Medical Biotechnology Commons

\section{Recommended Citation}

Lozano-Lewis, Lissette I., "Organometallic Rhenium Dyes for Nitric Oxide Detection and Imaging" (2015). FIU Electronic Theses and Dissertations. 2306.

https://digitalcommons.fiu.edu/etd/2306

This work is brought to you for free and open access by the University Graduate School at FIU Digital Commons. It has been accepted for inclusion in FIU Electronic Theses and Dissertations by an authorized administrator of FIU Digital Commons. For more information, please contact dcc@fiu.edu. 


\title{
FLORIDA INTERNATIONAL UNIVERSITY
}

Miami, Florida

\section{ORGANOMETALLIC RHENIUM DYES FOR NITRIC OXIDE DETECTION AND IMAGING}

\author{
A dissertation submitted in partial fulfillment of the \\ requirements for the degree of \\ DOCTOR OF PHILOSOPHY \\ in \\ CHEMISTRY \\ by \\ Lissette Irene Lozano-Lewis
}

2015 
To: Dean Michael R. Heithaus

College of Arts and Sciences

This dissertation, written by Lissette Irene Lozano-Lewis, and entitled Organometallic Rhenium Dyes for Nitric Oxide Detection and Imaging, having been approved in respect to style and intellectual content, is referred to you for judgement.

We have read this dissertation and recommended that it be approved.

$\begin{array}{r}\hline \text { Jaroslava Miksovska } \\ \hline \text { J. Martin Quirke } \\ \hline \text { Yong Cai } \\ \hline \text { Nikolaos Tsoukias }\end{array}$

Konstantinos Kavallieratos, Major Professor

Date of Defense: November 10, 2015

The dissertation of Lissette Irene Lozano-Lewis is approved

$\begin{array}{r}\begin{array}{r}\text { Dean Michael R. Heithaus } \\ \text { College of Arts and Sciences }\end{array} \\ \hline \begin{array}{r}\text { Dean Lakshmi N. Reddi } \\ \text { University Graduate School }\end{array}\end{array}$

Florida International University, 2015 
(C) Copyright 2015 by Lissette Irene Lozano-Lewis

All rights reserved 


\section{DEDICATION}

I dedicate this dissertation to my parents Jaime \& Irene for their immense love and for always being there with me, to my husband Joseph for having the patience and understanding of my vocation, and to my grandma Julia for guiding my steps from heaven. 


\section{ACKNOWLEDGMENTS}

I wish to thank Dr. Kavallieratos, my advisor, for his guidance throughout this graduate school experience, and all the members of my committee for their support and patience. Their gentle, but firm direction has been most appreciated. Dr. Miksovska was very helpful in instructing me in all the photophysical background and also allowing me access to her laboratory to perform some key fluorescence lifetime experiments. Dr. Nikolaos Tsoukias and Dr. Kumpal Madrasi collaborated with us in the cell fluorescence microscopy studies. I would like to thank Dr. Kevin O’Shea and his research group for access to the photoreactor apparatus for photochemical reactions. I also would like to thank Dr. Daniel Rabinovich, my former mentor at UNC-Charlotte for his continuing mentorship, friendship, and research collaboration on Chapter 4 of this work (Re- $\alpha$ diimine complexes). Finally, I would like to thank all of Dr. Kavallieratos's group members, past and present, for the patience and good humor, as they made my lab time really enjoyable.

I have found my coursework throughout the graduate curriculum and institutional programs to be stimulating and thoughtful, providing me with the tools to explore and solve both past and present challenges. 
ABSTRACT OF THE DISSERTATION

\section{ORGANOMETALLIC RHENIUM DYES FOR NITRIC OXIDE DETECTION AND \\ IMAGING}

by

Lissette Irene Lozano-Lewis

Florida International University, 2015

Miami, Florida

\section{Professor Konstantinos Kavallieratos, Major Professor}

The importance of sensing Nitric Oxide (NO) in physiology and medicine has led us to explore the reactivity of NO with organometallic Re dyes. Rhenium complexes were synthesized with the ability to react with $\mathrm{NO}$ and sense it under physiological conditions. Fluorescent 1,10-phenantroline complexes (phen) $\mathrm{Re}\left(\mathrm{PPh}_{3}\right)(\mathrm{CO})_{2} \mathrm{OSO}_{2} \mathrm{CF}_{3}$ (1) and (phen) $\mathrm{Re}\left(\mathrm{CH}_{3} \mathrm{CN}\right)(\mathrm{CO})_{2} \mathrm{OSO}_{2} \mathrm{CF}_{3}$ (3) can sense $\mathrm{NO}$ in the range of 10 - $150 \mu \mathrm{M}$ showing a decrease in fluorescence response at $514 \mathrm{~nm}$ and $532 \mathrm{~nm}$ respectively, upon NO-donor addition $\left(\lambda_{\text {exc }}=360 \mathrm{~nm}\right)$. (phen)Re(THF)(CO) ${ }_{2} \mathrm{OSO}_{2} \mathrm{CF}_{3}$ (2) showed a ratiometric response with a decrease in fluorescence intensity at $585 \mathrm{~nm}$ and an increase at $445 \mathrm{~nm}$. These complexes were characterized by FT-IR, ${ }^{1} \mathrm{H}-\mathrm{NMR},{ }^{13} \mathrm{C}-\mathrm{NMR}$, and ${ }^{19} \mathrm{~F}$-NMR. The quantum yields $(\Phi)$ and lifetimes $(\tau)$ were also determined. On the basis of ${ }^{19} \mathrm{~F}$-NMR and ${ }^{1} \mathrm{H}$-NMR spectroscopic evidence, the proposed mechanism of action involves dissociation of $\mathrm{CF}_{3} \mathrm{SO}_{3}{ }^{-}$(abbreviated as $\mathrm{OTf}^{-}$) together with $\mathrm{PPh}_{3}$, (for 1), THF (for 2), or $\mathrm{CH}_{3} \mathrm{CN}$ (for 3). Fluorescence microscopy showed that 1 permeates through the cell membrane of rat aortic endothelial cells (RAOEC) and 2 gives increase in cell fluorescence response at $450 \mathrm{~nm}$ when exogenous NO is added to RAOEC. The 
Re complexes of $\operatorname{dppz}$ (dppz = 2,2'-dipyrido[3,2-a:2,3'-c]phenazine) (dppz) $\mathrm{Re}(\mathrm{THF})(\mathrm{CO})_{2} \mathrm{OSO}_{2} \mathrm{CF}_{3}(\mathrm{~A} 2)$ and $\left.(\mathrm{dppz}) \mathrm{Re}\left(\mathrm{CH}_{3} \mathrm{CN}\right)(\mathrm{CO})_{2} \mathrm{OSO}_{2} \mathrm{CF}_{3}\right)$ (A3) were synthesized using the same methods as for complexes 1 and 3. Even though the dppz complexes exhibited similar behavior than the phenanthroline analogs, the signal changes were small and the results were inconclusive. The $\alpha$-Diimine Re complexes of Xy-DAD $\quad(\mathrm{XyDAD}=$ 1,4-bis-[2,6-dimethylphenyl]-1,4-diazo-1,3-butadiene $) \quad(\mathrm{Xy}-$ $\mathrm{DAD}) \mathrm{Re}(\mathrm{THF})(\mathrm{CO})_{2} \mathrm{OSO}_{2} \mathrm{CF}_{3} \quad$ (8) and $(\mathrm{Xy}-\mathrm{DAD}) \mathrm{Re}\left(\mathrm{CH}_{3} \mathrm{CN}\right)(\mathrm{CO})_{2} \mathrm{OSO}_{2} \mathrm{CF}_{3}$ showed reactivity with $\mathrm{NO}$ and $\mathrm{CN}^{-}$with a response in the visible range of the absorption spectrum. These diazadiene (DAD) complexes showed decreased reactivity with NO and $\mathrm{CN}^{-}$in the presence of oxidants. The $i$-PrDAD complexes $(i$-PrDAD $=1,4$-bis-[2,6diisopropylphenyl]-1,4-diazo-1,3-butadiene) (i-Pr-DAD)Re(THF)(CO) ${ }_{2} \mathrm{OTf} \quad(10)$ and (i-Pr-DAD)Re( $\left(\mathrm{CH}_{3} \mathrm{CN}\right)(\mathrm{CO})_{2} \mathrm{OTf}$ (11) exhibited similar behavior with the Xy-DAD analogs, but the spectral changes were much smaller and the results were inconclusive. The ESI-MS Mass Spectrometry studies of the phenanthroline complexes 1, and 2, after reaction with NO-donor showed that 1 and 2 react with $\mathrm{NO}$ to give $\left[\mathrm{Re}(\mathrm{CO})_{2}(\mathrm{NO})_{2} \mathrm{~S}_{2}\right]^{+}$ species, while (phen) $\mathrm{Re}(\mathrm{CO})_{3} \mathrm{Cl}$ and (phen) $\mathrm{Re}(\mathrm{CO})_{3} \mathrm{OSO}_{2} \mathrm{CF}_{3}$ do not show any metalNO containing products, which is consistent with the fluorescence studies. 


\section{TABLE OF CONTENTS}

1. INTRODUCTION

1.1. BIOLOGICAL ROLES OF NITRIC OXIDE 1

1.2. NITRIC OXIDE QUANTIFICATION 3

1.3. FLUORESCENT DYES FOR NO SENSING 4

1.3.1. Dyes based on NO-induced diamine cyclization process 4

1.3.1.1. Diaminofluorescein (DAF) 5

$\begin{array}{ll}\text { 1.3.2. } & \text { Dyes based on NO-induced diazo-ring-generation }\end{array}$

1.3.3. Dyes based on NO-induced spirolactam-ring-opening process 8

1.3.4. Dyes based on NO-triggered ligand dissociation from a metal complex 9

1.3.5. Dyes based on NO-triggered reduction of $\mathrm{Cu}^{+2}$ and release of fluorescent ligand 10

1.3.6. Dyes based on NO-induced spirolactam-ring-opening, reduction of $\mathrm{Cu}^{+2}$, and nitrosylation of fluorescent ligand

1.3.7. Dyes based on NO coordination to a transition metal and detachment of a fluorophore

1.4. USE OF METALS IN BIOLOGICAL SYSTEMS

$\begin{array}{ll}\text { 1.4.1. Metal complexes with fluorescent properties } & 14\end{array}$

1.4.2. Metal complexes for NO release 14

1.5. FAMILIES OF NO-DONORS 16

1.5.1. Organic nitrates 16

1.5.2. Organic nitrites 16

1.5.3. Metal-NO complexes 17

1.5.4. N-Nitrosamines 17

$\begin{array}{ll}\text { 1.5.5. N-hydroxy-N-Nitrosamines } & 17\end{array}$

$\begin{array}{ll}\text { 1.5.6. N-Nitrosimines } & 17\end{array}$

$\begin{array}{ll}\text { 1.5.7. N-Nitrosothiols (RSNOs) } & 18\end{array}$

$\begin{array}{ll}\text { 1.5.8. C-Nitroso compounds } & 18\end{array}$

1.5.9. Diazetine dioxides 18

1.5.10. Furoxans and benzofuroxans 18

$\begin{array}{lr}\text { 1.5.11. Oxatriazole-5-imines } & 19\end{array}$

$\begin{array}{lr}\text { 1.5.12. Sidnonimines } & 19\end{array}$

1.5.13. Oximes 19

1.5.14. Hydroxylamines 19

1.5.15. N-Hydroxyguanidines 19

1.5.16. Hydroxyurea 20

1.5.17. Zwitterionic polyamine/NO adducts (NONO-ate) 20

1.6. PROJECT RATIONALE 21

1.7. REFERENCES 24

2. (phen) $\mathrm{Re}\left(\mathrm{PPh}_{3}\right)(\mathrm{CO})_{2} \mathrm{OSO}_{2} \mathrm{CF}_{3}$ AS AN ORGANOMETALLIC FLUORESCENT NO SENSOR. 
2.1. ABSTRACT 35

2.2. INTRODUCTION 35

2.3. EXPERIMENTAL SECTION 37

2.3.1. Materials and Methods 37

2.3.2. Synthesis of (phen) $\mathrm{Re}(\mathrm{CO})_{2}\left(\mathrm{PPh}_{3}\right) \mathrm{OSO}_{2} \mathrm{CF}_{3} \quad 39$

2.3.3. Fluorescence studies with addition of NO-donor 40

2.3.4. Fluorescence titrations with gradual addition of NO-donor under anaerobic and aerobic conditions. 41

2.3.5. Fluorescence titrations with low concentrations of NO-donor. $\quad 42$

2.3.6. ${ }^{1} \mathrm{H}-\mathrm{NMR}$ and ${ }^{19} \mathrm{~F}-\mathrm{NMR}$ study of reactivity of Re complexes with NO 42

2.3.7. Fluorescence microscopy studies of (phen) $\mathrm{Re}\left(\mathrm{PPh}_{3}\right)(\mathrm{CO})_{2} \mathrm{OSO}_{2} \mathrm{CF}_{3}$ in Rat Aortic Endothelial Cells:

2.4. RESULTS AND DISCUSSION 44

2.4.1. Synthesis of (phen) Re( $\left(\mathrm{PPh}_{3}\right)(\mathrm{CO})_{2} \mathrm{OSO}_{2} \mathrm{CF}_{3} \quad 44$

2.4.2. Fluorescence studies with addition of NO-donor 45

2.4.3. Fluorescence titrations with gradual addition of NO-donor under anaerobic and aerobic conditions 48

2.4.4. Fluorescence titration with low concentrations of NO-donor. 52

2.4.5. FT-IR spectroscopy $\quad 55$

2.4.6. ${ }^{1} \mathrm{H}-\mathrm{NMR}$ and ${ }^{19} \mathrm{~F}-\mathrm{NMR}$ Study of (phen)Re( $\left(\mathrm{PPh}_{3}\right)(\mathrm{CO})_{2} \mathrm{OSO}_{2} \mathrm{CF}_{3}$ reactivity 57

2.4.7. Cell Studies \& Fluorescence Microscopy 58

2.5. CONCLUSIONS 58

2.6. REFERENCES 59

3. (phen) $\mathrm{Re}(\mathrm{THF})(\mathrm{CO})_{2} \mathrm{OSO}_{2} \mathrm{CF}_{3}$ AS A FLUORESCENT

ORGANOMETALLIC COMPLEX NO SENSOR. 62

3.1. ABSTRACT 62

3.2. INTRODUCTION 63

3.3. EXPERIMENTAL SECTION 65

3.3.1. Material and Methods 65

3.3.2. Conversion of (phen) $\mathrm{Re}(\mathrm{CO})_{3} \mathrm{OSO}_{2} \mathrm{CF}_{3}$ to (phen) $\mathrm{Re}(\mathrm{CO})_{2}$ (THF)OSO $\mathrm{OSF}_{3} \quad 66$

3.3.2.1. Method 1 - Thermal Substitution 66

3.3.2.2. Method 2 - Photochemical Substitution 67

3.3.3. Fluorescence studies with addition of NO-donor 68

3.3.4. ${ }^{1} \mathrm{H}-\mathrm{NMR}$ and ${ }^{19} \mathrm{~F}-\mathrm{NMR}$ study of reactivity of Re complexes with NO 68

3.3.5. Fluorescence microscopy studies of addition of $\mathrm{NO}$ to (phen) $\mathrm{Re}(\mathrm{THF})(\mathrm{CO})_{2} \mathrm{OSO}_{2} \mathrm{CF}_{3}$ in Rat Aortic Endothelial Cells 69

3.4. RESULTS AND DISCUSSION 71

$\begin{array}{ll}\text { 3.4.1. Design Considerations } & 71\end{array}$

$\begin{array}{ll}\text { 3.4.2. Fluorescence Studies } & 71\end{array}$

$\begin{array}{lll}\text { 3.4.3. } & \text { FT-IR spectroscopy } & 78\end{array}$

3.4.4. ${ }^{1} \mathrm{H}-\mathrm{NMR}$ and ${ }^{19}$ F-NMR Study of Re complex reactivity 80

3.4.5. Cell Studies \& Fluorescence Microscopy 84 
3.5. CONCLUSIONS 86

3.6. REFERENCES 86

4. (phen) $\mathrm{Re}\left(\mathrm{CH}_{3} \mathrm{CN}\right)(\mathrm{CO})_{2} \mathrm{OSO}_{2} \mathrm{CF}_{3}$ AS AN ORGANOMETALLIC

FLUORESCENT NO SENSOR $\quad 89$

4.1. ABSTRACT 89

4.2. INTRODUCTION 89

4.3. EXPERIMENTAL SECTION 90

4.3.1. Materials and Methods 90

4.3.2. Synthesis of (phen) $\mathrm{Re}\left(\mathrm{CH}_{3} \mathrm{CN}\right)(\mathrm{CO})_{2} \mathrm{OSO}_{2} \mathrm{CF}_{3}$

4.3.3. Fluorescence experiments with NO-donor 92

4.3.4. Fluorescence titrations with gradual addition of NO-donor under aerobic and anaerobic conditions 93

4.4. RESULTS 93

4.4.1. Synthesis of (phen) $\mathrm{Re}\left(\mathrm{CH}{ }_{3} \mathrm{CN}\right)(\mathrm{CO})_{2} \mathrm{OSO}_{2} \mathrm{CF}_{3}$ (3) 93

4.4.2. Fluorescent studies with addition of NO-donor 94

4.4.3. Fluorescence titrations with gradual addition of NO-donor under aerobic and anaerobic conditions 95

$\begin{array}{ll}\text { 4.5. CONCLUSION } & 97\end{array}$

4.6. REFERENCES 97

5. $\alpha$-DIIMINE RHENIUM COMPLEXES, AS COLORIMETRIC SENSORS $\begin{array}{ll}\text { FOR NO and } \mathrm{CN}^{-} & 99\end{array}$

5.1. ABSTRACT 99

5.2. INTRODUCTION 99

5.3. EXPERIMENTAL PROCEDURES 102

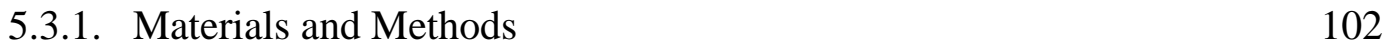

5.3.2. Synthesis of (Xy-DAD)Re(CO) ${ }_{3} \mathrm{Cl}(4) \quad 103$

5.3.3. Synthesis of (Xy-DAD) $\mathrm{Re}(\mathrm{CO})_{3} \mathrm{OSO}_{2} \mathrm{CF}_{3}(5)$

5.3.4. Synthesis of (i-Pr-DAD)Re(CO) ${ }_{3} \mathrm{Cl}(6) \quad 104$

5.3.5. Synthesis of (i-Pr-DAD)Re(CO) ${ }_{3} \mathrm{OSO}_{2} \mathrm{CF}_{3}(7)$

5.3.6. Photochemical substitution reactions 105

5.3.6.1. Synthesis of (Xy-DAD)Re(THF)(CO) ${ }_{2} \mathrm{OSO}_{2} \mathrm{CF}_{3}(8)$

5.3.6.2. Synthesis of (Xy-DAD)Re( $\left(\mathrm{CH}_{3} \mathrm{CN}\right)(\mathrm{CO})_{2} \mathrm{OSO}_{2} \mathrm{CF}_{3}(9) \quad 106$

5.3.6.3. Synthesis of (iPr-DAD)Re(CO) ${ }_{2} \mathrm{OSO}_{2} \mathrm{CF}_{3}(10)$

5.3.6.4. Synthesis of (iPr-DAD)Re(CO) ${ }_{2} \mathrm{OSO}_{2} \mathrm{CF}_{3}(11) 106$

5.3.7. Reactivity of $\alpha$-diimine complexes with NO-donor 107

5.3.8. Reactivity of $\alpha$-diimine complexes with $\mathrm{NaCN} \quad 107$

5.3.9. Comparison studies with other ions 108

5.4. RESULTS 108

5.4.1. Synthesis of (R-DAD)Re(L)(CO) ${ }_{2} \mathrm{OSO}_{2} \mathrm{CF}_{3}(\mathrm{R}=\mathrm{Me}$ or $i$-Pr; $\mathrm{L}=\mathrm{THF}$ or $\left.\mathrm{CH}_{3} \mathrm{CN}\right) \quad 108$

5.4.2. Reactivity of $\alpha$-diimine complexes with NO-donor 109

5.4.3. Reactivity of $\alpha$-diimine complexes with NaCN 111

5.4.4. Comparison studies with other ions 114 
5.5. CONCLUSIONS

5.6. REFERENCES

6. CONCLUSION

APPENDICES

VITA

145 


\section{TABLE OF FIGURES}

FIGURE

PAGE

Figure 2.1 Fluorescence Response upon NO-donor addition (0-150 $\mu \mathrm{M})$ to $20 \mu \mathrm{M}$ of 1 in acetone/PBS aqueous buffer $25 / 75 \mathrm{v} / \mathrm{v}$. $\lambda_{\text {exc }}=360 \mathrm{~nm}$ 45

Figure 2.2 Observed Fluorescence intensities of $1(20 \mu \mathrm{M})$ at $\lambda$ max $=514 \mathrm{~nm}$ upon NO-donor addition vs addition of control solution $(\mathrm{NaOH}$ $0.04 \mathrm{M}) . \lambda_{\text {exc }}=360 \mathrm{~nm}$.

Figure 2.3 Fluorescence Response of 1 ( $25 \mu \mathrm{M}$ - prepared photochemically, in THF) upon NO-donor addition (0-150 $\mu \mathrm{M})$, in acetone/PBS aqueous buffer $25 / 75 \mathrm{v} / \mathrm{v}$. $\lambda_{\text {exc }}=360 \mathrm{~nm}$.

Figure $2.4 \quad$ Fluorescence intensity ratio of $454 \mathrm{~nm} / 562 \mathrm{~nm}$ for $1(25 \mu \mathrm{M})$ with NO-donor addition $(0-150 \mu \mathrm{M})$

Figure 2.5 Fluorescence titration under nitrogen of $1(10 \mu \mathrm{M})$ with NO-donor $(0-150 \mu \mathrm{M})$ in acetone/buffer $25: 75$. $\lambda_{\text {exc }}=360 \mathrm{~nm}$.

Figure 2.6 Fluorescence titration under air of $1(10 \mu \mathrm{M})$ with NO-donor $(0-150 \mu \mathrm{M})$ in acetone/buffer 25:75. $\lambda_{\text {exc }}=360 \mathrm{~nm}$.

Figure 2.7 Fluorescence Titrations of $1(10 \mu \mathrm{M})$ with NO-donor $(0-300 \mu \mathrm{M})$, under $\mathrm{N}_{2}$ vs. under air. I514nm vs. NO-donor concentration.

Figure 2.8 Fluorescence Titrations of 1, prepared photochemically in THF from a $10 \mu \mathrm{M}$ solution of (phen) $\mathrm{Re}(\mathrm{CO})_{3} \mathrm{OSO}_{2} \mathrm{CF}_{3}$, with $\mathrm{NO}$-donor $(0-300 \mu \mathrm{M})$, under $\mathrm{N}_{2}$ vs. under air. I514nm vs. NO-donor concentration.

Figure 2.9 Fluorescence Spectra of $25 \mu \mathrm{M} 1$ (thermally prepared) with NO, acetone/buffer $25: 75, \lambda_{\text {exc }}=360 \mathrm{~nm}$, under nitrogen.

Figure 2.10 Observed fluorescence intensities of $25 \mu \mathrm{M} 1$ (thermally prepared) with gradual addition of NO-donor, acetone/buffer 25:75, $\lambda_{\text {exc }}=360 \mathrm{~nm}$

Figure 2.11 Fluorescence Spectra of $25 \mu \mathrm{M} 1$ (photochemically prepared) with NO, acetone/buffer 25:75, $\lambda_{\text {exc }}=360 \mathrm{~nm}$, under nitrogen.

Figure 2.12 Observed fluorescence intensities of $25 \mu \mathrm{M} 1$ (photochemically prepared) with gradual addition of NO-donor, acetone/buffer 


$$
25: 75, \lambda_{\text {exc }}=360 \mathrm{~nm}
$$

Figure 2.13 FT-IR spectrum of (phen) $\mathrm{Re}(\mathrm{CO})_{2}\left(\mathrm{PPh}_{3}\right) \mathrm{OSO}_{2} \mathrm{CF}_{3}(1)$

Figure 2.14 FT-IR spectrum of 1 after reaction with NO-donor 56

Figure 2.15 ${ }^{1} \mathrm{H}-\mathrm{NMR}$ spectra in $\mathrm{CDCl}_{3}$ (aromatic region) of 1 before (a) and after (b) reaction with NO-donor.

Figure 2.16 Fluorescence response of $1(10 \mu \mathrm{M})$ in RAOEC at $\lambda_{\mathrm{em}}=450 \mathrm{~nm}$ $\left(\lambda_{\text {exc }}=350 \mathrm{~nm}\right)$ showing the absorption of 1 into living cells.

Figure 3.1 Fluorescence Response upon NO-donor addition $(0-150 \mu \mathrm{M})$ to $20 \mu \mathrm{M}$ of 2 (generated by photochemical substitution) in acetone/PBS aqueous buffer $25 / 75 \mathrm{v} / \mathrm{v}$. $\lambda_{\text {exc }}=360 \mathrm{~nm}$.

Figure 3.2 Observed Fluorescence intensities $\lambda_{\max }=450 \mathrm{~nm}$ and $\lambda_{\max }=582 \mathrm{~nm}$ upon NO-donor addition on 2. [2] $=40 \mu \mathrm{M}, \lambda_{\text {exc }}=360 \mathrm{~nm}$.

Figure 3.3 Fluorescence Response upon NO-donor addition $(0-150 \mu \mathrm{M})$ to $20 \mu \mathrm{M}$ of Re complex 2 (generated by thermal substitution) in acetone/PBS aqueous buffer $25 / 75 \mathrm{v} / \mathrm{v}$. $\lambda_{\text {exc }}=360 \mathrm{~nm}$.

Figure 3.4 Fluorescence Spectra of $2(25 \mu \mathrm{M})$ with NO, acetone/buffer $25: 75, \lambda_{\text {exc }}=360 \mathrm{~nm}$, under nitrogen

Figure 3.5 Fluorescence Spectra of $2(25 \mu \mathrm{M})$ with NO, acetone/buffer $25: 75, \lambda_{\text {exc }}=360 \mathrm{~nm}$

Figure 3.6 Fluorescence Spectra of $2(30 \mu \mathrm{M})$ with NO, acetone/buffer 25:75, $\lambda_{\text {exc }}=360 \mathrm{~nm}$. Comparison of spectra collected under $\mathrm{N}_{2}$ vs in air. 77

Figure 3.7 Fluorescence intensity ratio of $430 \mathrm{~nm} / 578 \mathrm{~nm}$ for $2(25 \mu \mathrm{M})$ with NO-donor addition $(0-150 \mu \mathrm{M})$

Figure 3.8 FT-IR spectrum of 2

Figure $3.9 \quad$ FT-IR spectrum of 2 after reaction with NO-donor and wash with $1 \mathrm{M} \mathrm{HCl}$ followed by $1 \mathrm{M} \mathrm{NaHCO}_{3}$

Figure 3.10 Aromatic region of ${ }^{1} \mathrm{H}-\mathrm{NMR}$ spectra (d8-THF) for 2 (top), 2 after $30 \mathrm{~min}$ of UV irradiation at $300 \mathrm{~nm}$ (middle), and after $90 \mathrm{~min}$ of UV irradiation (bottom).

Figure 3.11 Aromatic region of ${ }^{1} \mathrm{H}-\mathrm{NMR}$ spectra after reaction with NO-donor 
Figure 3.12 Aromatic region of ${ }^{1} \mathrm{H}$-NMR spectra after reaction of 2 with NO-donor ( $\mathrm{d}_{8}$-THF) (a) before and (b) after wash with $1 \mathrm{M} \mathrm{HCl}$ followed by $1 \mathrm{M} \mathrm{NaHCO}_{3}$.

Figure 3.13 Picture sequence demonstrating fluorescence response in RAOEC at $\lambda_{\mathrm{em}}=450 \mathrm{~nm}$, Dye was generated by photochemical substitution (50 min). (a) with no NO-donor added (b) with $1 \mu \mathrm{M}$ NO-donor (c) with $5 \mu \mathrm{M}$ NO-donor and (d) with $10 \mu \mathrm{M}$ NO-donor. $\lambda_{\text {exc }}=350 \mathrm{~nm}$.

Figure 4.1 Fluorescence Response upon NO-donor addition $(0-150 \mu \mathrm{M})$ to $20 \mu \mathrm{M}$ of Re complex 3 in acetone/PBS aqueous buffer $25 / 75 \mathrm{v} / \mathrm{v}$, $\left(\lambda_{\text {exc }}=360 \mathrm{~nm}\right)$

Figure 4.2 Fluorescence Spectra of $50 \mu \mathrm{M} 3$ with NO, acetone/buffer 25:75, $\lambda_{\text {exc }}=360 \mathrm{~nm}$, under nitrogen

Figure 4.3 Fluorescence Spectra of $50 \mu \mathrm{M} 3$ with NO, acetone/buffer 25:75, $\lambda_{\text {exc }}=360 \mathrm{~nm}$

Figure 4.4 Fluorescence Spectra of $1150 \mu \mathrm{M}$ with NO, comparison nitrogen vs air at $532 \mathrm{~nm}$

Figure 5.1 Interaction of $(\mathrm{Xy}-\mathrm{DAD}) \mathrm{Re}(\mathrm{CO})_{2}(\mathrm{THF}) \mathrm{OSO}_{2} \mathrm{CF}_{3}$ with NO-donor, acetone/PBS (1:1)

Figure 5.2 Relationship and ratio of the reaction of (Xy-DAD)

$\mathrm{Re}(\mathrm{CO})_{2}$ (THF) $\mathrm{OSO}_{2} \mathrm{CF}_{3}$ with NO-donor, acetone/PBS (1:1)

Figure $5.3 \quad$ Interaction of $(\mathrm{Xy}-\mathrm{DAD}) \mathrm{Re}(\mathrm{CO})_{2}(\mathrm{MeCN}) \mathrm{OSO}_{2} \mathrm{CF}_{3}$ with NO-donor, Acetone/PBS (1:1)

Figure 5.4 Interaction of (Xy-DAD)Re(CO) $)_{2}(\mathrm{THF}) \mathrm{OSO}_{2} \mathrm{CF}_{3}$ with $\mathrm{NaCN}$, acetone/PBS (1:1)

Figure 5.5 Interaction of $(\mathrm{Xy}-\mathrm{DAD}) \mathrm{Re}(\mathrm{CO})_{2}\left(\mathrm{CH}_{3} \mathrm{CN}\right) \mathrm{OSO}_{2} \mathrm{CF}_{3}$ with $\mathrm{NaCN}$, acetone/PBS (1:1)

Figure 5.6 Relationship and ratio of the reaction of (Xy-DAD) $\mathrm{Re}(\mathrm{CO})_{2}\left(\mathrm{CH}_{3} \mathrm{CN}\right) \mathrm{OSO}_{2} \mathrm{CF}_{3}$ with $\mathrm{NaCN}$, acetone/PBS (1:1) 114 
Figure 5.7 Comparison Studies among (Xy-DAD) $\mathrm{Re}(\mathrm{THF})(\mathrm{CO}){ }_{2} \mathrm{OSO}_{2} \mathrm{CF}_{3}$

with NO-donor and Different Ions at $500 \mathrm{~nm}$

Figure 5.8 Comparison Studies among (Xy-DAD)Re(MeCN)(CO) ${ }_{2} \mathrm{OSO}_{2} \mathrm{CF}_{3}$ with NO-donor and Different Ions at $500 \mathrm{~nm}$

Figure A1.1 Energy diagram for (a) $\left[(\mathrm{dppz}) \mathrm{Re}(\mathrm{CO})_{3}(\mathrm{py})\right] \mathrm{PF}_{6}$ and (b) (dppz) $\mathrm{Re}(\mathrm{CO})_{3} \mathrm{Cl}$

Figure A1.2 Fluorescence Response upon NO-donor addition (0-150 $\mu \mathrm{M})$ to $25 \mu \mathrm{M}$ of Re complex A2 in acetone/PBS aqueous buffer $25 / 75 \mathrm{v} / \mathrm{v}$, $\left(\lambda_{\text {exc }}=360 \mathrm{~nm}\right)$

Figure A1.3 Fluorescence Response upon NO-donor addition (0-150 $\mu \mathrm{M})$ to $25 \mu \mathrm{M}$ of Re complex A3 in acetone/PBS aqueous buffer 25/75 v/v, $\left(\lambda_{\text {exc }}=380 \mathrm{~nm}\right)$

Figure A1.4 UV-Visible Spectra of A4 $25 \mu \mathrm{M}$ after addition of different amounts of NO, in acetone/buffer 25:75

Figure A1.5 UV-Visible absorbance of A4 $25 \mu \mathrm{M} 6$ vs NO concentration, in acetone/buffer 25:75

Figure A1.6 UV-Visible absorbance of A2 $25 \mu \mathrm{M} 6$ vs NO concentration, in acetone/buffer 25:75

Figure A1.7 UV-Visible absorbance of A3 $25 \mu \mathrm{M} 6$ vs NO concentration, in acetone/buffer 25:75

Figure A2.1 ESI-MS positive mode spectrum of (phen)Re(CO)2(THF)OTf

Figure A2.2 ESI-MS positive mode spectrum of (phen) $\mathrm{Re}(\mathrm{CO})_{2}\left(\mathrm{PPh}_{3}\right) \mathrm{OTf}$

Figure A2.3 ESI-MS positive mode spectrum of (phen) $\operatorname{Re}(\mathrm{CO})_{3} \mathrm{Cl}$

Figure A2.4 ESI-MS positive mode spectrum of (phen)Re(CO) ${ }_{3} \mathrm{OTf}$ 


\section{TABLE OF SCHEMES}

SCHEME

PAGE

Scheme 1.1 Stress-induced EDRF-NO mechanism

Scheme 1.2 Mechanism of NO fluorescent sensors based on NO-induced diamine cyclization process

Scheme 1.3 DAF-FM mechanism of action toward $\mathrm{NO} / \mathrm{O}_{2} \mathrm{O}_{2}$ in the absence of extracellular leaking 6

Scheme 1.4 Frontier orbital energy diagram: Illustration of the thermodynamic situation of the fluorescence OFF/ON switch including the PET process

Scheme 1.5 Mechanism of NO fluorescent sensors based on NO-induced diazoring generation process

Scheme 1.6 Mechanism of NO fluorescent sensors based on NO-induced spirolactam-ring opening process

Scheme 1.7 Mechanism of NO fluorescent sensors based on NO-triggered dissociation of a fluorescence ligand from a metal complex

Scheme 1.8 Mechanism of NO fluorescent sensors based on NO-triggered reduction of $\mathrm{Cu}^{+2}$ and release of fluorescent ligand

Scheme 1.9 Dyes based on NO-induced spirolactam ring-opening, reduction of $\mathrm{Cu}^{+2}$, and nitrosylation of fluorescent ligand

Scheme 1.10 Lippard's Co(II) complex used as NO sensor

Scheme 1.11 UV-Visible transitions observed for $(\mathrm{L}) \operatorname{Re}(\mathrm{CO})_{3} \mathrm{X}$

Scheme 1.12 Structure of a generic zwitterionic NONOates

Scheme 1.13 Proposed mechanism for the ratiometric response for proposed Re-based NO sensors

Scheme 2.1 Synthesis of (phen) Re( $\left.\mathrm{PPh}_{3}\right)(\mathrm{CO})_{2} \mathrm{OSO}_{2} \mathrm{CF}_{3}(1)$ 
$\begin{array}{lll}\text { Scheme 3.1 Concept diagram for Re-phen NO sensors } & 71\end{array}$

Scheme 3.2 Proposed mechanistic pathways for reactivity of 2 with NO. 86

Scheme 4.1 Synthesis of (phen) $\mathrm{Re}\left(\mathrm{CH}_{3} \mathrm{CN}\right)(\mathrm{CO})_{2} \mathrm{OSO}_{2} \mathrm{CF}_{3}(3)$

Scheme 5.1 Valence tautomerization (VT) produced by bistable electron state of DAD

Scheme 5.2 Summary of synthesis of metal-DAD complexes: a. from metal halide or carbonyl; b. from chelating ligand substitution; c. from Schiff-base condensation

Scheme 5.3 Synthetic path for DAD-rhenium complexes

Scheme A1.1 Synthesis of (dppz)Re(L)(CO) ${ }_{2} \mathrm{OSO}_{2} \mathrm{CF}_{3}$ 


\section{ACRONYMS AND ABBREVIATIONS}

\begin{tabular}{|c|c|}
\hline $\mathrm{CN}^{-}$ & Cyanide \\
\hline $\mathrm{CO}$ & Carbon monoxide \\
\hline DAD & 1,4-diazo-1,3-diene \\
\hline dppz & 2, 2’-dipyrido[2,3 -a:2’,3’-c]phenazine \\
\hline EDRF & Endothelial Derived Relaxation Factor \\
\hline ESI-MS & Electrospray Ionization Mass Spectrometry \\
\hline FT-IR & Fourier Transform Infrared Spectroscopy \\
\hline$i-\operatorname{Pr}$ & Isopropyl \\
\hline$\lambda_{\mathrm{em}}$ & Emission wavelength \\
\hline$\lambda_{\text {exc }}$ & Excitation wavelength \\
\hline M & Molarity (mol/L) \\
\hline $\mathrm{MeCN}$ & Acetonitrile \\
\hline${ }^{3} \mathrm{MLCT}$ & Triplet - Metal to ligand charge transfer \\
\hline$\mu \mathrm{M}$ & micromolar \\
\hline $\mathrm{mM}$ & millimolar \\
\hline NMR & Nuclear Magnetic Resonance \\
\hline NO & Nitric Oxide \\
\hline $\mathrm{OSO}_{2} \mathrm{CF}_{3}^{-}$ & $\mathrm{CF}_{3} \mathrm{SO}_{3}^{-}$(trifate) \\
\hline PBS & Phosphate Buffer Saline \\
\hline phen & 1,10-phenanthroline \\
\hline
\end{tabular}


$\mathrm{PPh}_{3}$

RAOEC

THF

UV-Vis

Xy
Triphenyl Phosphine

Rat Aortic Endothelial Cells

Tetrahydrofurane

Ultra Violet - Visible

Xylene

\section{INTRODUCTION}

Nitric oxide, or oxidonitrogen (NO), ${ }^{1}$ is a free radical produced as byproduct of nitrogenized fossil fuels and electrical discharges. Nitric Oxide is catalogued as an oxidizer and will both support and propagate combustion. Because of the presence of oxygen, $\mathrm{NO}$ is converted into $\mathrm{NO}_{2}$ rapidly in air. Nitric Oxide is converted to $\mathrm{HNO}_{2}$, nitrous acid, in aqueous solutions in the presence of oxygen.

\subsection{BIOLOGICAL ROLES OF NITRIC OXIDE}

The importance of NO in biology and physiology was highlighted in 1998 when Drs. Furchgott, Murad and Ignarro won the Nobel Prize in medicine and physiology for their research in NO physiology. Currently, there are over 5,000 publications per year that deal with the role of NO in biomedicine.

In biological systems, NO is involved in signaling and several physiological and pathological processes. Nitric Oxide is short-lived in the blood and is known as an endothelial derived relaxing factor (EDRF) because of its vasodilator properties. ${ }^{3}$ Nitric Oxide is produced in cells by the combined role of three isoforms of Nitric Oxide Synthase (NOS) enzymes: i) endothelial (eNOS or NOS-3), ii) neuronal (nNOS or NOS1) and iii) inducible (iNOS or NOS-2), the last of which is calcium-dependent. The process involves arginine oxidation followed by the reduction of molecular oxygen. ${ }^{4}$ 
Nitric Oxide is involved in important functions in the smooth muscle, such as muscle relaxation, vasodilatation, and regulation of blood flow. ${ }^{5}$ Nitric Oxide is also involved in neuronal activities acts as neurotransmitter. ${ }^{6}$ It is important in the immune system's attack against pathogens and neoplasia. ${ }^{7}$ Homeostasis of NO in biological systems is a key component of the cellular health. Nowadays, there is increasing research in exogenous NO sources. ${ }^{8}$ There is a vast selection of novel compounds that release NO (or NO-like substrates) to specific targets under special triggers (like nitrites under hypoxic conditions). ${ }^{9} \quad$ Nitric oxide promotes the generation of the messenger cyclic 3',5'-guanosine monophosphate (cGMP), activating the nucleotide-dependent protein kinase $\mathrm{G}$ (PKG or cGKI). Protein kinase $\mathrm{G}$ is an enzyme involved in the phosphorylation of several calcium regulators. ${ }^{10}$ Actin-myosin dynamic alterations derive from varying calcium concentration-sensitization and hyperpolarizing potassium channels, which leads to smooth muscle relaxation (Scheme 1.1). ${ }^{10}$ Nitric Oxide functions as an immune system booster, killing invasive bacteria by oxidative damage. ${ }^{11}$ However, in extreme cases, these increments in intracellular NO concentration lead to profuse vasodilation, causing the hypotension characteristics in sepsis cases or reaching cytotoxic levels leading to an apoptotic cascade. ${ }^{12}$ 


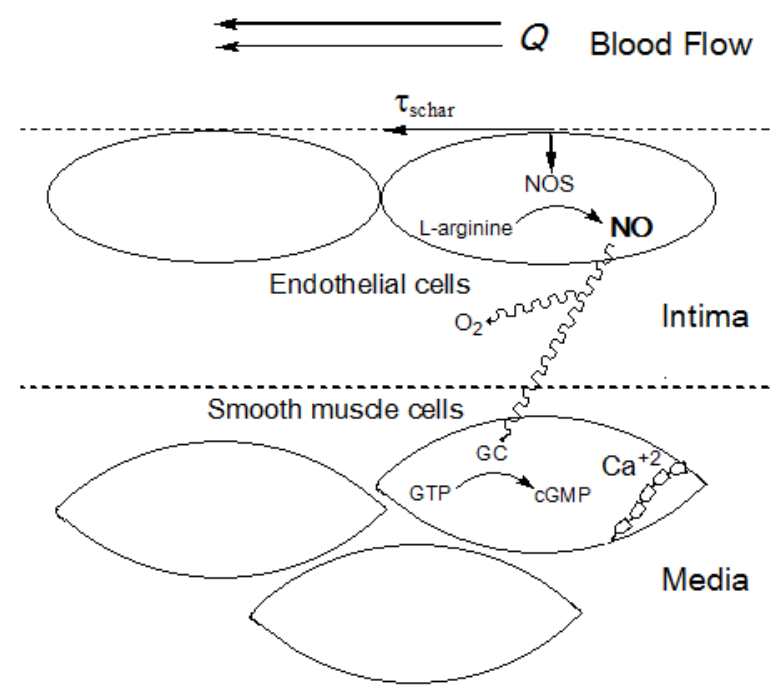

Scheme 1.1 Stress-induced EDRF-NO mechanism ${ }^{10}$

Because of its relatively low polarity, NO is less soluble in oxygen-free water (1.9 $\mathrm{mM})$ than in organic solvents, such as methanol (14 mM), toluene $(11 \mathrm{mM})$, and hexane $(18 \mathrm{mM}) .{ }^{13}$ Nitric Oxide is sufficiently lipophilic to permeate through cell membranes. It is oxidized to $\mathrm{NO}_{2}^{-}$in oxygen-containing aqueous solutions with a rate constant ranging from $3 \times 10^{6} \mathrm{M}^{2} \mathrm{~s}^{-1}$ to $12 \times 10^{6} \mathrm{M}^{2} \mathrm{~s}^{-1}{ }^{14} \quad$ The NO oxidation reaction is 300 times faster in aqueous conditions than in hydrophobic conditions. ${ }^{15}$ Micromolar concentrations of NO can trigger formation of reactive nitrogen species, which produces cell damage, carcinogenesis, and neurodegenerative disorders. ${ }^{16}$

\subsection{NITRIC OXIDE QUANTIFICATION}

The quantification of $\mathrm{NO}^{17}$ in tissues remains a challenge because of the absence of a detection method that can combine significant spatial and temporal resolution with high NO specificity. ${ }^{18}$ The challenges lie in NO's short lifetime $\left(\mathrm{t}_{1 / 2}=\mathrm{ca} .5 \mathrm{~s}\right)$ and the fact that it is found in low concentrations in living tissues. 
Detection methods for NO include chemiluminescence, electrochemistry, and spin-trapping followed by EPR (Electron Paramagnetic Resonance). Chemiluminescence is produced when NO is exposed to ozone; ${ }^{19}$ the limitation of this technique is the difficulty of adding the analytes to the detector, making it unsuitable for monitoring NO in biological systems. Another method uses luminol, ${ }^{20}$ which has the same response as peroxides even though it is less cytotoxic than using hydrogen peroxide. Electrochemical microsensors can provide real-time detection; ${ }^{21}$ but spatial information is limited to the electrode tip, making it unsuitable for NO-related biochemistry studies. EPR has been proposed for studies in vitro and in vivo. ${ }^{22}$ However, as a result of its high reactivity and low concentration, NO cannot be reliably quantified by EPR techniques. In order to improve the EPR technique, spin-trapping reagents can be used to form stable radicals, giving enough time for detection; There are different types of NO spin trapping reagents: nitrone (DMPO) and nitroso (MNP, DBNBS), which are used in cell cultures; ${ }^{23}$ nitrosylhemoprotein, ferrous-Hb; ${ }^{24}$ nitronyl nitroxides; ${ }^{25}$ Dithiocarbamates/Irondithiocarbamate (DTC/Fe-DTC), which is the only type of spin trapping reagent used in vivo; ${ }^{26 a}$ Dithiocarbamates have antioxidant properties and react with NO almost at the diffusion-controlled rate. ${ }^{27 b}$

\subsection{FLUORESCENT DYES FOR NO SENSING}

Fluorescence imaging is a form of epifluorescence or confocal microscopy in which using objective lens focuses the excitation ray going through a pinhole, limiting the section of the sample to analyze. ${ }^{27}$ Fluorescent dyes have been used for quantification and imaging of NO at inter- and intracellular concentrations. ${ }^{28}$ The advantage of such dyes is that they can be irradiated at specific intensities and emissive dye-substrate 
complexes formed upon reaction with NO can be observed. Some fluorescent dyes have high selectivity and can quantify NO at sub-micromolar concentrations. These dyes can be separated into six different groups: i) Dyes based on NO-Induced diamine cyclization process; ii) dyes based on NO-Induced diazo-ring-generation process; iii) based on NOInduced spirolactam-ring-opening; iv) dyes based on NO-Triggered selective ligands dissociation of Metal complex; v) dyes based on NO-Triggered reduction of copper(II) and release of fluorescent ligand; vi) dyes based on NO-Induced spirolactam ringopening, reduction of $\mathrm{Cu}^{+2}$, and nitrosylation of fluorescent ligand.

\subsubsection{Dyes based on NO-induced diamine cyclization process:}

NO sensing by these dyes is based on formation of a highly fluorescent triazole in the presence of $\mathrm{N}_{2} \mathrm{O}_{3}{ }^{29}$ (oxidized product of $\mathrm{NO}$ in presence of oxygen). The change in response can be attributed to the less electron donating group triazole, disturbing the PET process (Photo-Electron Transfer) recovering the fluorescence intensity. The core of this type of fluorescent dye is shown in Scheme 2:
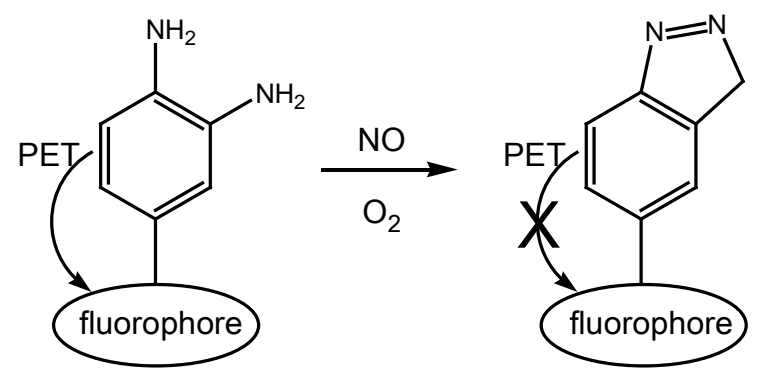

Scheme 1.2 Mechanism of NO fluorescent sensors based on NO-induced diamine cyclization process $^{17 \mathrm{~b}}$

These sensors contain different types of fluorophores: i) Fluoresceine, ${ }^{30}$ e.g., DAF and DAF-FM; ii) BODIPY ${ }^{31}$ (boron dipyrromethene complexes that have stability in large ranges of $\mathrm{pH}$ ); iii) Acridine; ${ }^{32}$ iv) Anthraquinone; ${ }^{33}$ v)Tricarbocyanine, ${ }^{34}$ (highly 
soluble in water near infrared luminescent probes, designed for human tissue NO imaging); vi) Phosphorescent heavy-metal-complexes as lunimophores, ${ }^{35}$ (e.g., ruthenium-bipy core). Using this mechanism ratiometric sensors can also be developed, in which the ratiometric sensing is realized through the fluorescence resonance energy transfer (FRET) process; ${ }^{30 d}$ The carbon dots serve as both the energy donor and the anchoring site for the NO probe moiety, which turns into the energy acceptor in the presence of NO.

\subsubsection{Diaminofluorescein (DAF)}

Since 1995, the Nagano group has been developing fluorometric methods to detect NO using diaminonaphthalene (DAN). In the presence of oxygen DAN yields the fluorescent naphthalene triazole (NAT) under neutral conditions. ${ }^{36}$ Fluorescence intensity increases incrementally with NO concentration. However, DAN cannot be used for quantification in biological systems because it leaks out through the cell membrane. Using the same methodology, Diaminofluorescein (DAF) was shown to react with $\mathrm{NO} / \mathrm{O}_{2}$, producing DAF-T (triazole) with a small change in absorbance and a large increase in fluorescence (180-fold). DAF is suitable for cell studies; its excitation wavelength is in the visible range, not making auto-fluorescence possible. ${ }^{37}$ Different series of diaminofluorescein dyes have been synthesized in order to modify the $\mathrm{pK}_{\mathrm{a}}$, increase quantum yield, increase stability in the presence of $\mathrm{pH}$ changes, increase cell membrane permeability, decrease autofluorescence, decrease photobleaching, and increase cell trappability of the DAF-T complex. 

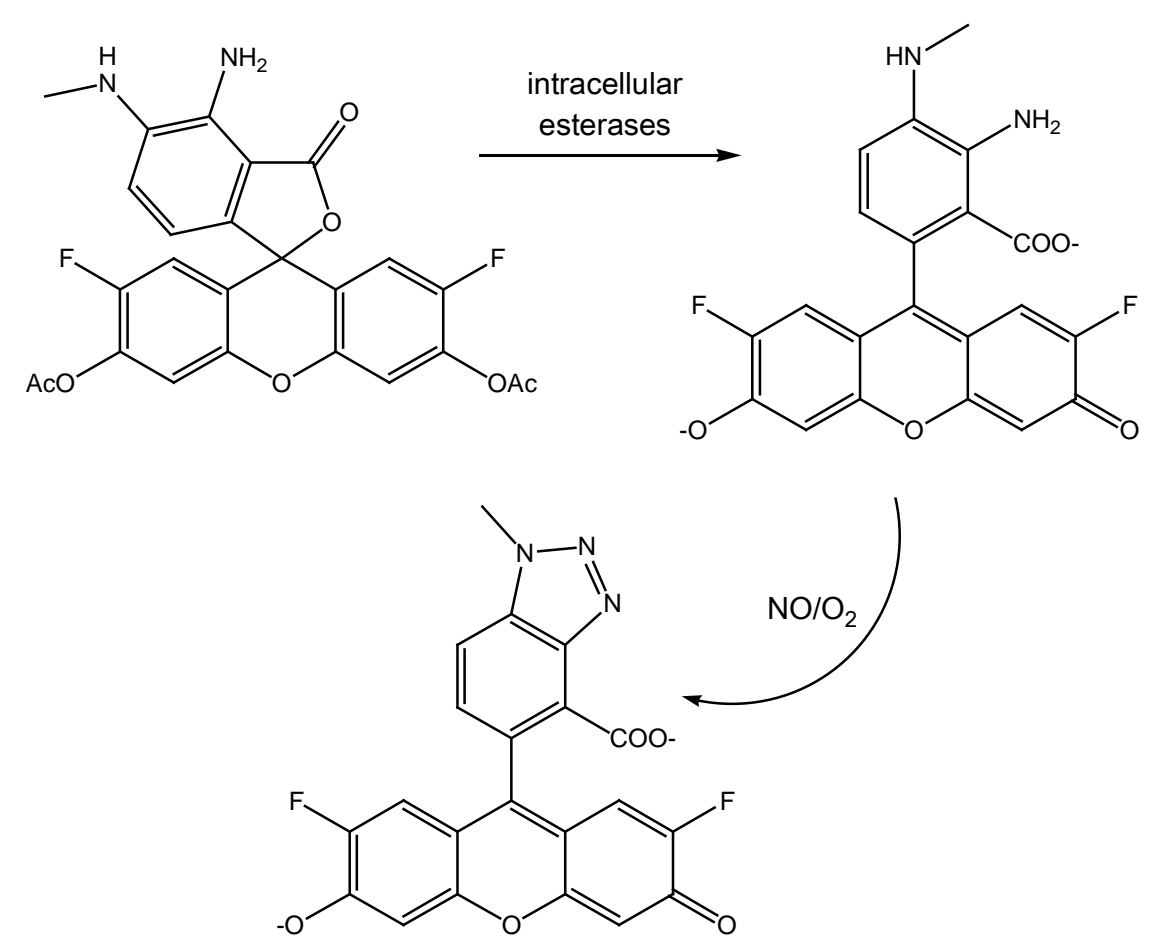

Scheme 1.3 DAF-FM mechanism of action toward $\mathrm{NO} / \mathrm{O}_{2}$ in the absence of extracellular leaking. ${ }^{38}$

Fluorinated DAFs were shown to be more resistant to photobleaching; DAF-FM is the most sensitive and stable within the DAF series, ${ }^{38}$ with an excitation wavelength of $488 \mathrm{~nm}$ and emission wavelength of $515 \mathrm{~nm}$. It is the most widely used fluorescent probe for NO imaging. Even though the mechanism remains unclear, the fluorescence enhancement is ascribed to the interruption of photoinduced electron transfer (PET) between the benzoic acid and the xanthene moiety. ${ }^{39}$ Kinetic studies of NO reactivity with DAF-FM, by Tsoukias et al. have shown that the dye response depends on $\mathrm{N}_{2} \mathrm{O}_{3}$ concentration. ${ }^{39 d}$

Photoinduced electron transfer is a widely accepted mechanism for fluorescence quenching. The fluorescein structure has two parts: the benzoic acid moiety as the PET donor and the xanthene ring as the fluorophore. The electron transfer from the PET donor 
decreases the fluorescence intensity of xanthene. The small variations between excitation and emission wavelengths of DAF and DAF-T can be attributed to the dihedral angle between the benzoic acid moiety and the xanthene ring being almost $90^{\circ}$, as shown in the X-ray structures of analogous compounds. ${ }^{39 c}$

Weakly fluorescent

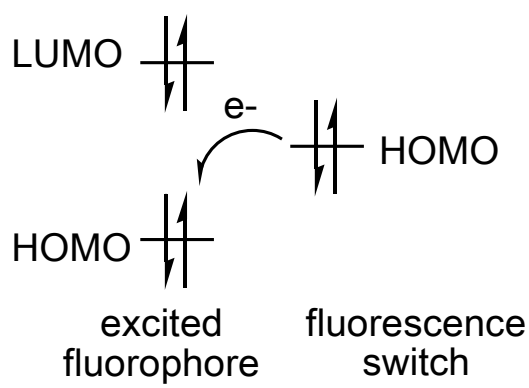

Strongly fluorescent

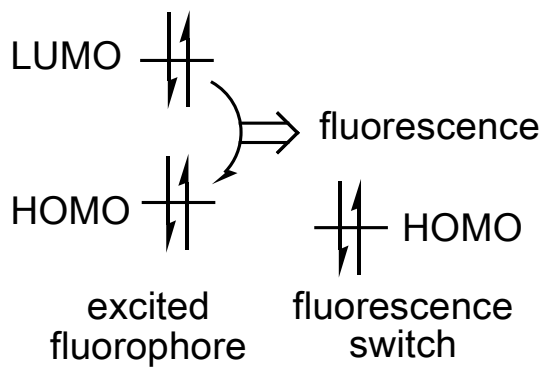

Scheme 1.4 Frontier orbital energy diagram: Illustration of the thermodynamic situation of the fluorescence OFF/ON switch including the PET process ${ }^{39}$

\subsubsection{Dyes based on NO-induced diazo-ring-generation}

These fluorophores react with NO, forming a rigid $\pi$-conjugation system that enhances the fluorescence intensity up to three orders of magnitude, ${ }^{40}$ as shown in Scheme 1.5. These dyes have low cytotoxicity and cross the cell membranes, but not the nuclear membrane. Anslyn and coworkers have developed a dye which in the presence of $\mathrm{NO} / \mathrm{N}_{2} \mathrm{O}_{3}$ generates a diazo-ring fluorophore. The dye reacts with $\mathrm{NO}$ forming an N-nitrosoaniline intermediate, which then undergoes electrophilic substitution to form the diazo- product. The probe has preference for $\mathrm{NO} / \mathrm{N}_{2} \mathrm{O}_{3}$ over hydroxyl radical, nitrite, nitrate and peroxynitrite. The product generated is stable in a wide range of $\mathrm{pH}$ and the fluorescence intensity does not increase over time. 
<smiles>CN(C)c1ccc2nnc3c4cc([N+](=O)[O-])cc(N(C)C)c4ccc3c2c1</smiles>

Scheme 1.5 Mechanism of NO fluorescent sensors based on NO-induced diazo-ring generation process. ${ }^{40}$

\subsubsection{Dyes based on NO-induced spirolactam-ring-opening process:}

These sensors use rhodamine as fluorophore and a reactive lactam group. ${ }^{41}$ After reacting with $\mathrm{NO}$, the lactam ring opens and fluorescence intensity increases because of the extension in the conjugation of the rhodamine fluorophore. The dual fluorophore system is used as the Förster resonance energy transfer (FRET) dyad. There is a change in the conjugated system that allows the turn on/off of both dyes. The system is selective toward NO over other competitive radicals and ions. Although this dye is efficient, its synthesis and purification is complex.
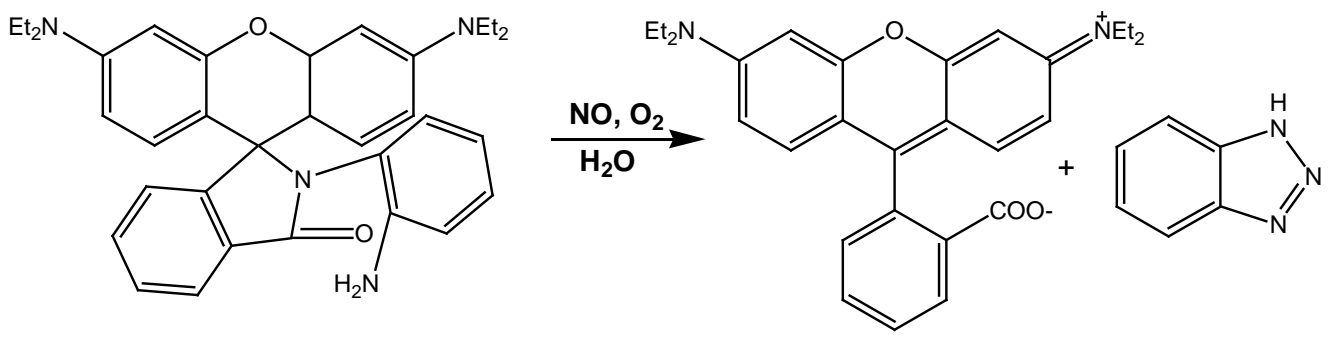

Scheme 1.6 Mechanism of NO fluorescent sensors based on NO-induced spirolactam-ring opening process

\subsubsection{Dyes based on NO-triggered ligand dissociation from a metal complex}

Metal to ligand charge transfer (MLCT) between the partially filled d-orbitals of a metal and the excited states of the fluorophore can cause fluorescence quenching. 
Formation of a Metal-NO complex displaces the fluorophore and turns on the fluorescence emission (Scheme 1.7). Using this mechanism of action, Co(II), ${ }^{42} \mathrm{Rh}(\mathrm{II}),{ }^{43}$ $\mathrm{Ru}(\mathrm{II}),{ }^{44} \mathrm{Fe}(\mathrm{II}),{ }^{45}$ and $\mathrm{Cu}(\mathrm{II})$ complexes have been designed for NO detection. Most of these complexes react directly with NO. However, they are insoluble or unstable in aqueous solutions because water displaces fluorophores from the coordination sphere or hydrolyses the fluorophores.

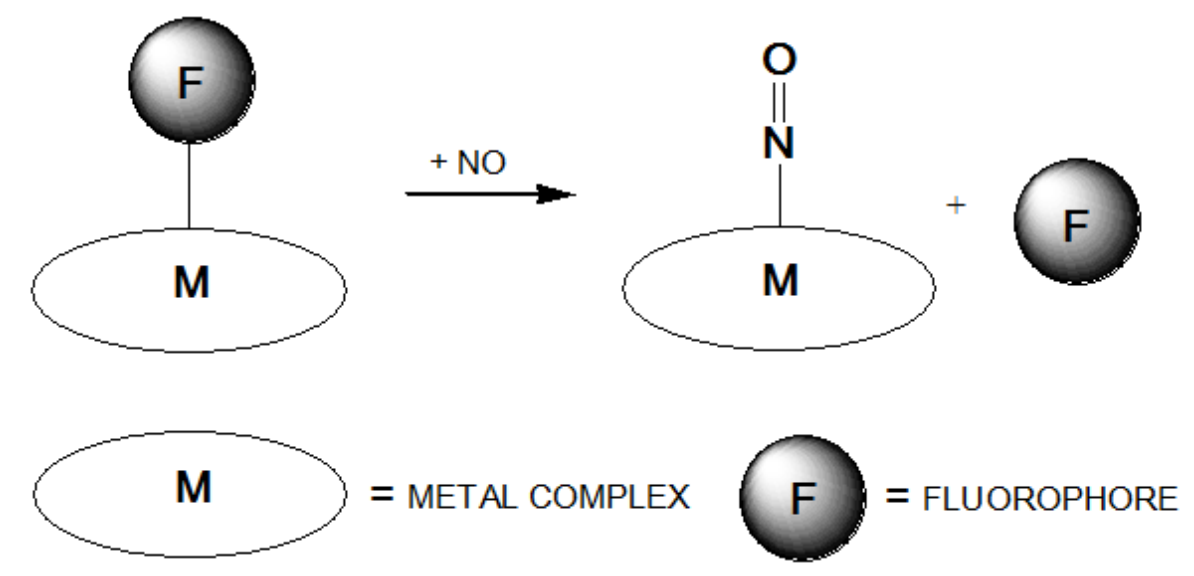

Scheme 1.7 Mechanism of NO fluorescent sensors based on NO-triggered dissociation of a fluorescence ligand from a metal complex

\subsubsection{Dyes based on NO-triggered reduction of $\mathrm{Cu}^{+2}$ and release of} fluorescent ligand:

Most metal nitrosyl complexes described in the previous section will slowly hydrolyze if their solutions are exposed to air for several days, as water can outcompete the fluorophore for coordination to the metal center. The metal nitrosyl hydrolysis side reaction is why a new type of dye was introduced using $\mathrm{Cu}(\mathrm{II})$ complexes for NO detection. The new strategy involves the formation of a $\mathrm{Cu}(\mathrm{I})$ species via NO-triggered reduction of paramagnetic $\mathrm{Cu}(\mathrm{II})$ that was associated with $\mathrm{NO}$ coordination to a $\mathrm{Cu}$ center and the release of the fluorescent nitrosylated ligand, corresponding to 
fluorescence enhancement. Lippard's group explored this idea by preparing a new probe that include a modified Cyclam ligand in the metal coordination sphere. ${ }^{46}$ Initially this probe was prepared to sense $\mathrm{Hg}(\mathrm{II})$.

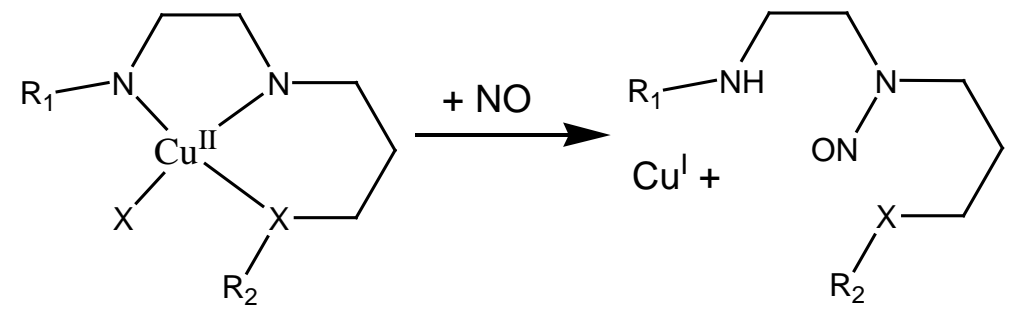

Scheme 1.8 Mechanism of NO fluorescent sensors based on NO-triggered reduction of $\mathrm{Cu}(\mathrm{II})$ and release of fluorescent ligand

Lippard et al. have developed a series of copper-fluorescein complexes that can detect NO under neutral aqueous conditions, in the absence of oxygen. ${ }^{47}$ The addition of hydrolysable esters or conjugation to dextran are used to add cell-trappability. ${ }^{48,49}$ The major improvement compared to DAF is that this dye responds to NO and not to an oxidized product of NO. Cu-FL is selective for NO over hypochlorite, superoxide, peroxide, nitrite, nitrate, peroxinitrite and HNO. The quantum yield of Cu-FL ( $\Phi=$ $0.077)$ is substantially lower than for fluorescein $(\Phi=0.95)$. After reaction with NO and elimination of $\mathrm{Cu}(\mathrm{I})$, the quantum yield increases again in the nitrosylated fluorescein ( $\Phi=0.58)$. Thus the mechanism is called turn-off/turn-on.

\subsubsection{Dyes based on NO-induced spirolactam ring-opening, reduction of} $\mathrm{Cu}^{+2}$, and nitrosylation of fluorescent ligand

Recently, there have been more examples of sensors that use the combination of two or more described mechanisms. In a recent example, by Duan and coworkers; the 
dye mode of action combines spirolactam ring opening and reduction of $\mathrm{Cu}(\mathrm{II}) .{ }^{50}$ The mechanism is similar to the one described in the previous section 1.3.5.<smiles>CCNc1ccc2c(c1)Oc1cc(N(CC)CC)ccc1C21c2ccccc2C(=O)N1CCN1CCNC(Cl)C1</smiles>

Scheme 1.9 Dyes based on NO-induced spirolactam ring-opening, reduction of $\mathrm{Cu}^{+2}$, and nitrosylation of fluorescent ligand ${ }^{50}$

\subsubsection{Dyes based on NO coordination to a transition metal and detachment}

\section{of a fluorophore}

When a fluorophore is coordinated to a transition metal, its intensity is suppressed because of the interaction with the partially filled d-orbitals. Under the presence of NO, NO displaces the fluorophore from the metal center, forming stable nitrosyl complexes and the fluorophore regains its initial fluorescence intensity. ${ }^{132}$ For example, there is the dansylated animotroponimate Co(II) complex prepared by Lippard ${ }^{133}$ in which a 4-fold increase in fluorescence intensity is observed in the presence of NO. The major challenge for these selective dyes is solubility in aqueous physiological media in order for them to be suitable for fluorescence cell microscopy. Many transition metal complexes do not pass this challenge. 


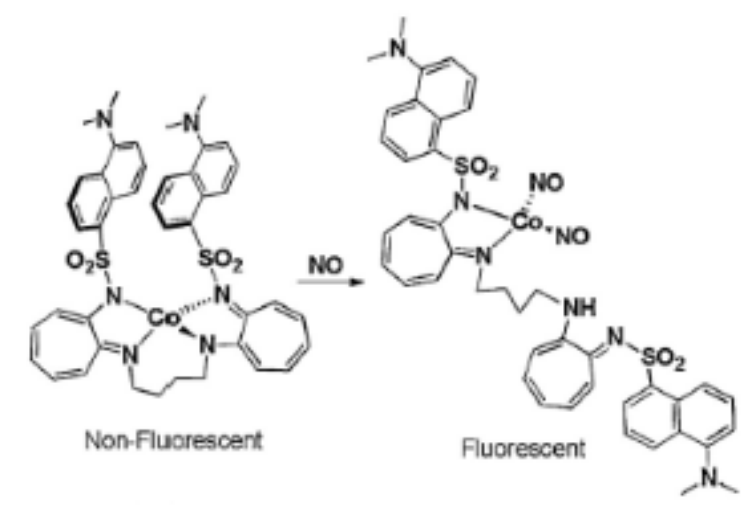

Scheme 1.10 Lippard's Co(II) complex used as NO sensor. (Figure reproduced from reference 133)

\subsection{USE OF METALS IN BIOLOGICAL SYSTEMS}

Metals are used in biological systems to perform several roles, such us active sites in enzymes, regulators, electrolytes, messengers, transport agents, and transmitters. ${ }^{51}$ They also provide accessible scaffolds important for structural stability. ${ }^{52}$ Table 1.1 provides a summary of these roles.

Table 1.1 Functional roles of inorganic elements applied to biology.

\begin{tabular}{cc}
\hline Function & $\begin{array}{c}\text { Inorganic } \\
\text { Element }\end{array}$ \\
\hline
\end{tabular}


Structure

Signaling

Acid-base catalysis

Luminescence

Magnetic resonance

Radioactive

X-ray

Alteration of metal bioavailability

Bonding

Ligand exchange

Electron transfer

Redox
Ru, Rh,

$\mathrm{Pt}$

Ca, NO

Co, Zr,

Pt, Ln

Tb, Eu, other Ln

Subcellular imaging agents

Gd, Mn Metal-responsive MRI imaging

${ }^{99 \mathrm{~m}} \mathrm{Tc},{ }^{64}$

$\mathrm{Cu}, \quad$ SPECT, PET imaging

others

Os, Ln

Heavy atom derivatives for biomolecular X-ray structure determination

$\mathrm{Fe}, \mathrm{Cu}$, $\mathrm{Zn}, \mathrm{Ca}$, other

Zn, Ni, Metal-ligands bond formation for analyte other sensing, metalloprotein inhibition, or protein labeling

Gd, Co, Change in coordination sphere to increase MRI others signal or release bioactive molecule

$\mathrm{Rh}, \mathrm{Ru}$

Photoinduced electron transfer to produce potent photooxidants

$\mathrm{Fe}, \mathrm{Cu}, \quad$ Oxidative degradation of proteins, DNA; others chelation to manipulate redox activity

The technicium isotope ${ }^{99 \mathrm{~m}} \mathrm{Tc}$ has been used for tumor imaging. ${ }^{53}$ By using a combination of photophysical and radiolabeling, cancer therapy and the treatment response can be monitored. ${ }^{54}$ These two elements have similar electronic properties. Technicium and rhenium form analogous complexes with similar physical and chemical 
properties, these complexes are expected to have the same pharmacokinetic behavior and are, therefore, considered a "matched pair" for diagnosis and therapy. ${ }^{99 m} \mathrm{Tc}$ biocomplexes can be used as an alternative to PET radioligands such as ${ }^{11} \mathrm{C},{ }^{55-56},{ }^{18} \mathrm{~F},{ }^{57}$ and ${ }^{124} \mathrm{I}^{58}$

\subsubsection{Metal complexes with fluorescent properties}

Fluorescence microscopy is a resourceful, high-resolution technique that can be used for imaging and the study of luminescent molecular probes. Metal complexes that exhibit MLCT usually possess long-lived excited states in the order of hundreds of nanoseconds to milliseconds. The long-lived excited state allows the use of time-resolved luminescence microscopy (TRLM) in order to eliminate autofluorescence. ${ }^{59}$ Metal complexes can have long Stokes shifts, low cytotoxicity, and high cellular uptake. Targeted intracellular localization can be achieved by carefully choosing the fluorophores. ${ }^{60}$ Several transition metals and lanthanides have been used as luminophores for in vivo imaging, including $\operatorname{Ir}(\mathrm{III}),{ }^{61} \mathrm{Pt}(\mathrm{II}),{ }^{62} \mathrm{Eu}(\mathrm{III}),{ }^{63}$ and $\mathrm{Tb}(\mathrm{III}) .{ }^{64}$

\subsubsection{Metal complexes for NO Release}

There are metal complexes that release NO after irradiation. ${ }^{65}$ The Fe-S-NO clusters, such as Roussin's salt, ${ }^{66}$ and simple Ru complexes, like $\left[\mathrm{Ru}(\mathrm{NO}) \mathrm{Cl}_{5}\right]^{2-}$, can be used for this purpose. $^{67}$ The $\mathrm{PaPy} \mathrm{H}^{\mathrm{H}}$ ( $N, N$-bis(2-pyridylmethyl)amine- $N$-ethyl-2pyridine-2-carboxamide) group was introduced by Mascharak and coworkers as a pentacoordinated ligand that can provide four equatorial nitrogen sites in an octahedral metal complex with an apical NO. ${ }^{68}$ The $\mathrm{Ru}(\mathrm{II})-\mathrm{PaPy}{ }_{3} \mathrm{H}$ complex can release $\mathrm{NO}$ and it is soluble and stable in aqueous conditions. ${ }^{69}$ The incorporation of fluorescent dyes into NO releasing compounds provides a path for tracking and monitoring targeted cellular 
NO uptake. ${ }^{70}$ The promotion of an electron from the metal molecular orbital to a $\pi^{*}$ (NO) antibonding orbital is required for the metal nitrosyl photoactivity. ${ }^{71}$ The NOreleasing complexes, which can be excited in the near infrared (NIR) region, are needed because of mammalian tissue penetration restriction. ${ }^{72}$

Rhenium tricarbonyl complexes have been used for imaging applications because of their luminescent properties provided by the ${ }^{3}$ MLCT: large Stokes shifts, fluorescent lifetimes in the hundreds of nanoseconds range, and good quantum yields. ${ }^{73}$ Lately, it has been discovered that these qualities also can be used in biological probes. ${ }^{74}$ In $(\mathrm{L}) \operatorname{Re}(\mathrm{CO})_{3} \mathrm{X}$ type complexes $(\mathrm{L}=$ bidentate nitrogen ligand with extended conjugated system), the UV-Vis spectrum shows three transitions (Scheme 1.11): (i) a high energy band $\pi \rightarrow \pi^{*}$, (ii) a lower MLCT energy band $\operatorname{Re} \rightarrow \pi^{*}(\mathrm{~L})$, and (iii) a $X \rightarrow L$, LLCT transition. ${ }^{75}$ These tricarbonyl complexes exhibit solvatochronism ${ }^{76}$ and are greatly affected by substitutions in the polypyridine ligand. These complexes possess a $\mathrm{C}_{\mathrm{s}}$ geometry with three active vibrational modes $\left[\mathrm{A}^{\prime}(1)+\mathrm{A}^{\prime}(2)+\mathrm{A}^{\prime \prime}\right]$ corresponding to the three CO in facial configuration. ${ }^{77}$

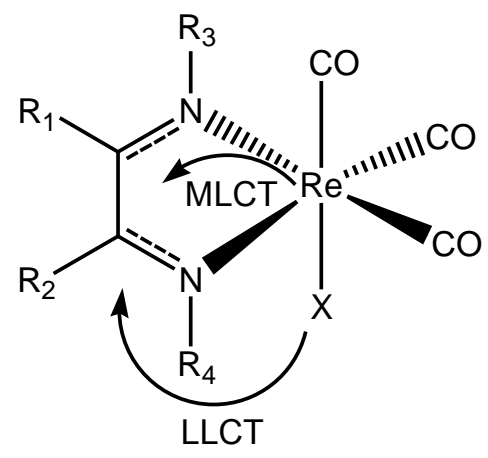

Scheme 1.11 UV-Visible transitions observed for $(\mathrm{L}) \operatorname{Re}(\mathrm{CO}){ }_{3} \mathrm{X}$

\subsection{FAMILIES OF NO-DONORS}


Nitric Oxide has a solubility in water of 2 - $3 \mathrm{mM}$ and reacts rapidly with several radicals and water. ${ }^{78}$ Some advantages of using NO-donors are: Ease of use vs. NO gas, targetted delivery, and production modulation in order to avoid side reactions. ${ }^{79}$ For almost 150 years, glyceryl trinitrate (GTN) has been used as an NO-donor for medical purposes. $^{80}$ There are seventeen different classes of NO-donors, which vary from enzymatic generators to redox generators. These NO-donors have different biological applications. $^{81}$

\subsubsection{Organic nitrates $\left(\mathrm{RONO}_{2}\right)$}

Organic nitrates are alcohol nitric acid esters. Organic nitrates are the oldest class, medically applied. They are synthesized directly from nitric acid and alcohol or from alkyl halide and $\mathrm{AgNO}_{3}{ }^{82}$ They have low solubility in water and they suffer from hydrolysis and $\beta-\mathrm{H}$ elimination in alkaline conditions. ${ }^{83}$ The biggest shortcoming is "nitrate tolerance"; even though the mechanism is not clear, it may result in sulfhydryl depletion. ${ }^{84}$

\subsubsection{Organic nitrites.}

These are esters of alcohols and nitrous acid. They have been widely used as vasodilators. They can be synthesized by esterification of alcohols with nitrous acid, ${ }^{85}$ reaction with other nitrosilating agents, or by transesterification. ${ }^{86}$ Organic nitrites have lower boiling points than the corresponding alcohols, and react rapidly in the presence of nucleophiles, transferring the nitrosyl group. ${ }^{87}$

\subsubsection{Metal-NO complexes}


This group has been discussed before (1.3.4-1.3.7). Sodium nitroprusside (SNP), dinitrosyl iron thiol complexes (DNICs), iron-sulfur clusters nitrosyl, ruthenium nitrosyls, and iron-porphyrin nitrosyls are members of the Metal-NO family.

\subsubsection{N-Nitrosamines}

$\mathrm{N}$-nitrosamines have been used sparingly as solvents or intermediates in the synthesis of hydrazines and diazoalkanes. ${ }^{88}$ They are considered carcinogenic because they can alkylate biomolecules. ${ }^{89}$

\subsubsection{N-hydroxy-N-Nitrosamines.}

N-hydroxy-N-Nitrosamines decompose to generate NO under physiological conditions. Therefore, they can be used as anti-hypertensive agents and inhibitors of platelet aggregation. ${ }^{90}$ They are prepared by direct nitrosation of N-hydroxyamines. ${ }^{91}$ Cupferron is used in extraction, separation, and colorimetric detection of metals. ${ }^{92}$ It releases NO under enzymatic, ${ }^{93}$ electrochemical, ${ }^{94}$ and oxidative conditions. ${ }^{95}$

\subsubsection{N-Nitrosimines.}

Heterocyclic compounds produced from the correspondent imines. There are seven subtypes according to the substituents. ${ }^{96}$ They release either $\mathrm{NO}$ or $\mathrm{N}_{2} \mathrm{O}$ from the decomposition of HNO. In the presence of thiols, the production of $\mathrm{N}_{2} \mathrm{O}$ is favored over NO.

\subsubsection{Nitrosothiols (RSNOs).}

Nitrosothiols were first synthesized in $1909 .{ }^{97}$ There are few RSNOs that are stable. They are proposed as storing, transfering and delivering vehicles for NO. They are synthesized from thiols and NO derivatives. ${ }^{98}$ The reaction rates with NO are influenced by steric effects; bulky substrates react slower than less hindered ones. ${ }^{99}$ 


\subsubsection{C-Nitroso compounds}

Tertiary C-nitroso compounds with germinal electron withdrawing groups can be used as NO-donors. These compounds are prepared from their corresponding hydroxylamines. ${ }^{100}$ They release NO under thermolysis and photolysis. ${ }^{101}$ In biological systems, they can produce in vitro vasodilation and decrease the presence of oxyhemoglobin. ${ }^{102}$

\subsubsection{Diazetine dioxides}

These four-member ring heterocycles were first introduced in $1971 .^{103}$ They decompose in physiological aqueous solutions. ${ }^{104}$ Diazetine dioxides were used to decrease the systolic high blood pressure in rats. ${ }^{105}$

\subsubsection{Furoxans and benzofuroxans.}

These five member heterocycles may lead to positive and negative biological outcomes. ${ }^{106}$ These compounds are thermally stable; they are reactive in presence of nucleophiles and bases. They release NO in presence of thiols ${ }^{107}$ and undergo ring opening reactions with biological thiols, such as cysteine or glutathione. ${ }^{108}$

\subsubsection{Oxatriazole-5-imines}

These mesoionic substituted five-member heterocycles were first reported in 1965. ${ }^{109}$ They have been used as blood pressure depressants. ${ }^{110}$ They were initially synthesized from monohydrazines through substituted cyanohydrazines. ${ }^{111}$

\subsubsection{Sidnonimines}


Sidnonimines is an important class of NO-donors ${ }^{112}$ introduced in $1957 .{ }^{113}$ These reagents produce stoichiometric amounts of superoxide anions; NO and superoxide react with each other, producing peroxynitrite. Their metabolites are potent vasorelaxants. ${ }^{114}$

\subsubsection{Oximes}

The alkyl- and aryl- oximes can be converted to NO under oxidative conditions. ${ }^{115}$ These compounds are vasoactive, with similar activity to NO. They are synthesized by addition of a nitro and a hydroxyimino group into a diene by a nitronitrosation step. ${ }^{116}$ Oximes decompose into NO and the corresponding ketone, under physiological conditions. ${ }^{117}$

\subsubsection{Hydroxylamines:}

Hydroxylamines are hygroscopic free-base products of mammalian cells. ${ }^{118}$ In alkaline solutions, they disproportionate into ammonia, nitrogen and nitrous oxide. Hydroxylamines can produce NO under enzymatic conditions. ${ }^{119}$

\subsubsection{N-Hydroxyguanidines}

$\mathrm{N}$-Hydroxyguanidines are products of guanidine and hydroxyurea that exhibit cytotoxicity against leukemic cells and antitumor activity in vivo. ${ }^{120}$ In contrast to other NO-donors, hydroxyguanidines do not produce NO spontaneously: They need NOS ${ }^{121}$ or P450 ${ }^{122}$ enzymes to produce NO.

\subsubsection{Hydroxyurea}

Hydroxyurea is a well-known antitumor agent. ${ }^{123}$ It shows some activity against some cancers, ${ }^{124}$ skin diseases, ${ }^{125}$ and inhibits HIV virus replication. ${ }^{126}$ In 1998, the first in vitro reaction of oxyhemoglobin with hydroxyurea was reported. It involved the 
specific transfer of NO from the $\mathrm{NHOH}$ group of hydroxyurea in biomolecules taken from sickle cell anemia patients. ${ }^{127}$

\subsubsection{Zwitterionic polyamine/NO adducts (NONOates)}

Bubbling $\mathrm{NO}$ to amines produces $\mathrm{RN}[\mathrm{N}(\mathrm{O}) \mathrm{NO}]^{-}(\mathrm{CH} 2)_{\mathrm{x}} \mathrm{NH}_{2}{ }^{+}{ }^{128}$ The half-lives at physiological conditions may vary from $1.8 \mathrm{~min}$ to $56 \mathrm{~h}$, depending on the amine substituents. ${ }^{129}$ These adducts are affected by slow decomposition even in the solid state, and thus are stored at low temperatures $\left(-78^{\circ} \mathrm{C}\right){ }^{130}$ NONOates may release two full equivalents of NO in solution ${ }^{131}$ and some have extended shelf life.

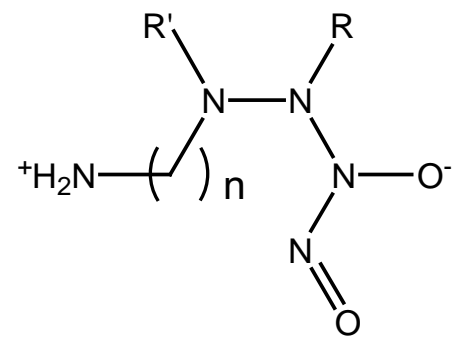

Scheme 1.12 Structure of a generic zwitterionic NONOate.

Table 1.2 Spectroscopic properties and NO-release half-lives for different NONOate type NO-donors. Measured in $0.01 \mathrm{M} \mathrm{NaOH}$. Determined at $22{ }^{\circ} \mathrm{C}$ and $\mathrm{pH} 7.4$ in $0.1 \mathrm{M}$ phosphate buffer.

\begin{tabular}{|c|c|c|c|c|c|}
\hline \multicolumn{6}{|c|}{$\mathbf{R N}[\mathbf{N}(\mathbf{O}) \mathbf{N O}]^{-}\left(\mathbf{C H}_{2}\right)_{\mathbf{x}} \mathbf{N H}_{\mathbf{2}}{ }^{+} \mathbf{R}^{\prime}$} \\
\hline $\mathbf{x}$ & $\mathbf{R}$ & $\mathbf{R}^{\prime}$ & $\begin{array}{c}\mathbf{U V} \boldsymbol{\lambda}_{\max } \\
\mathbf{( n m )}\end{array}$ & $\begin{array}{c}\boldsymbol{\varepsilon} \mathbf{x} \mathbf{1 0}^{-\mathbf{3}} \\
\left.\mathbf{( M}^{-1} \mathbf{c m}^{-\mathbf{1}}\right)\end{array}$ & $\mathbf{t}_{\mathbf{1} / \mathbf{2}}$ (min) \\
\hline 2 & $\mathrm{Me}$ & $\mathrm{Me}$ & 250 & 7.31 & 36.1 \\
\hline
\end{tabular}




\begin{tabular}{|r|c|c|c|c|c|}
3 & Me & Me & 250 & 7.68 & 10.1 \\
\hline 4 & Me & Me & 250 & 8.59 & 1.3 \\
\hline 6 & Me & Me & 250 & 7.25 & 3.7 \\
\hline 2 & Me & H & 250 & 7.8 & 40 \\
\hline 2 & Et & H & 250 & 7.96 & 333 \\
\hline 2 & Et & Et & 250 & 7.61 & 327 \\
\hline 3 & Me & H & 250 & 7.77 & 13.7 \\
\hline 3 & Et & Et & 250 & 8.55 & 71.8 \\
\hline 3 & Pr & H & 250 & 8.05 & 76.6 \\
\hline 3 & i-Pr & H & 250 & 7.44 & 93 \\
\hline 3 & i-Pr & i-Pr & 250 & 7.89 & 88.5 \\
\hline 3 & cyclohex & H & 250 & 9.13 & 115 \\
\hline 2 & $\left(\mathrm{CH}_{2}\right)_{2} \mathrm{NH}_{2}$ & H & 252 & 7.64 & 3400 \\
\hline 3 & $\left(\mathrm{CH}_{2}\right)_{3} \mathrm{NH}_{2}$ & H & 252 & 7.83 & 284 \\
\hline 3 & $\left(\mathrm{CH}_{2}\right)_{4} \mathrm{NH}_{2}$ & H & 250 & 9.42 & 165 \\
\hline
\end{tabular}

\subsection{PROJECT RATIONALE}

The objective of this project is the synthesis, characterization and NO sensing and imaging study of Re-based ratiometric fluorescent dyes. The unique mechanism we envisioned for $\mathrm{NO}$ sensing depends on change in fluorescence properties that occurs upon NO coordination. In order to obtain a ratiometric fluorescent probe that can detect changes in NO concentration with high sensitivity and selectivity, an entirely new approach on NO sensing is employed that combines the following elements: 1) NO binding to a metal center, such as $\operatorname{Re}(\mathrm{I})$; 2) a bidentate or tridentate phenathroline (phen), dipyridophenazine (dppz), or $\alpha$-diimine framework attached on the metal, which provides favorable photophysical characteristics, as well potential for modification; and 3) two weakly-coordinated ligands: i) The X-type $\mathrm{CF}_{3} \mathrm{SO}_{3}{ }^{-}$and ii) An L-type ligand, such as 
THF, $\mathrm{CH}_{3} \mathrm{CN}$, or $\mathrm{PPh}_{3}$. The sensing mechanism may involve the formation of nitrosyl complexes with concurrent alteration of photophysical properties of an organometallic complex upon reaction with NO.
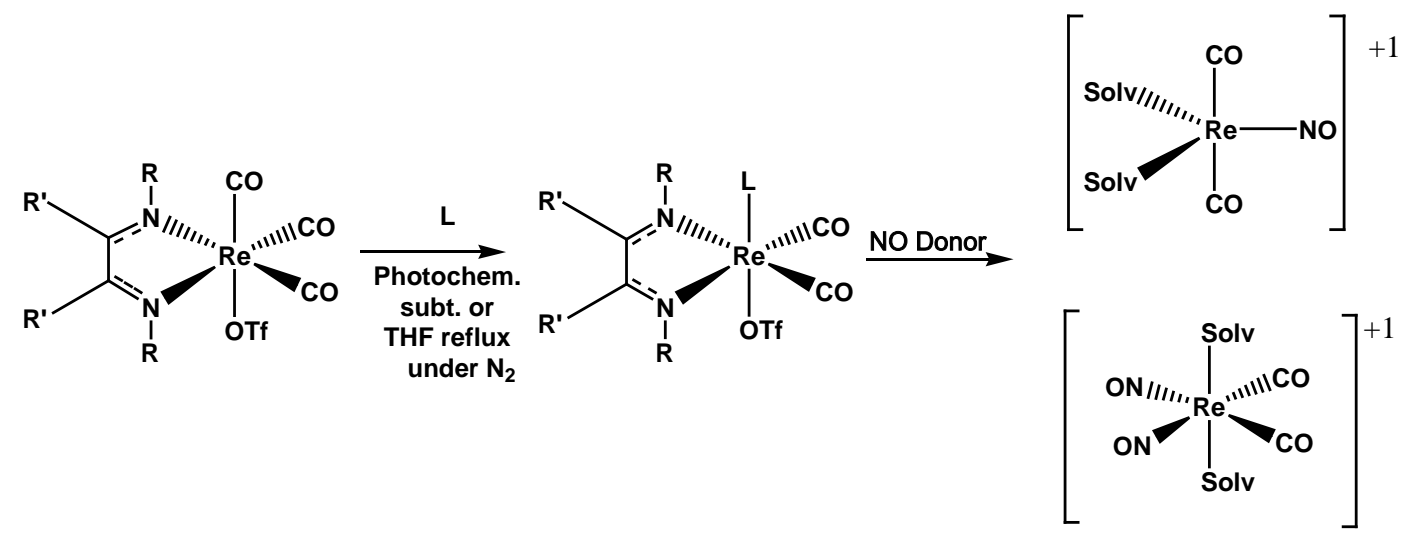

Scheme 1.13 Proposed mechanism for the ratiometric response for Re-based NO sensors.

The mechanism of NO sensing is based on the optical and photophysical properties of rhenium, and the affinity that rhenium has for Nitric Oxide (NO) to form nitrosyl complexes. These complexes sense $\mathrm{NO}$ in aqueous media, at $37^{\circ} \mathrm{C}$ and $\mathrm{pH} 7$ and are expected to be able to permeate the cell membrane and get trapped in the cytosolic media. The strong ${ }^{3} \mathrm{MLCT}$ is a result of the presence of carbonyl groups. Dissociation of labile ligands ( $\mathrm{L}$ and $\mathrm{CF}_{3} \mathrm{SO}_{3}{ }^{-}$) and $\mathrm{NO}$ attachment leads to a different fluorescence maximum, promoting ratiometric response. Aromatic ligands (polypyridines, phenanthrolines) can give this probe membrane cell permeability, providing the complex is neutral. Change in polarity or overall charge of the complex upon NO reaction could potentially trap the product inside the cell.

Previous attempts of rhenium phenanthroline complexes ${ }^{35 b}$ as NO sensors have used diaminopyridine, which transforms into a triazole in a mechanism similar to DAF family of dyes (1.3.1). In those dyes there was no coordination of NO to Re. 
Rhenium Tricarbonyl $\left[\operatorname{Re}(\mathrm{CO})_{3}(\mathrm{~N}-\mathrm{N})(\mathrm{X})\right]+$ systems: This type of kinetically inert $\operatorname{Re}(\mathrm{I})$ $\mathrm{d}^{6}$ 18-electron complexes displays a ${ }^{3} \mathrm{MLCT}$ phosphorescent emission after near visible ultra-violet and visible light excitation. The function of these carbonyl ligands is to avoid the deactivation of $\operatorname{Re}(\mathrm{I})$ metal center. The ${ }^{3} \mathrm{MLCT}$ transition is characteristic of $\mathrm{d}^{6}$ Rhenium(I)-carbonyl complexes. There are also non-carbonyl rhenium complexes that able to display this transition. However, their ligand field splitting is very small; for example, Re(bipy)з is non-luminescent. Several other ligands like polypyridines, bisquinolines and pyridazines have been studied as probes for cell imaging. ${ }^{134}$

Rhenium imaging probes have shown reliable responses. Changing fluorophores selectively may target different subcellular structures. Rhenium(I) reacts with specific biochemical agents localized in the cell, such as sulfur-containing aminoacids in the mitochondria, or with macromolecules by non-covalent interactions. None of these precursors uses NO-triggered selective ligand dissociation from a metal complex, as a mechanism to provide NO sensing with a ratiometric response. The proposed probe is expected to selectively sense NO even under anaerobic conditions, because it does not need $\mathrm{O}_{2}$ for activation. Several current $\mathrm{NO}$ dyes respond to NO indirectly with the real substrates being $\mathrm{N}_{2} \mathrm{O}_{3}$ or $\mathrm{NO}_{2}$ radical formed by reaction of $\mathrm{NO}$ with oxygen. The ReNO dyes we propose are soluble in aqueous media, (pH between 7.2 and 7.4), by using only a minimum amount of organic solvent. In order for the Re-NO dyes to meet requirements for widespread applicability, they need to be stable under physiological conditions, permeable through the cell membranes and modifiable to be trapped inside the cell.

\subsection{REFERENCES}


1. (a) "Nitric Oxide (CHEBI:16480)". Chemical Entities of Biological Interest (ChEBI). UK: European Bioinformatics Institute. (b) Nitric Oxide Material Safety Data Sheet (http://www.cdc.gov/niosh/docs/2003-154/pdfs/6014-1.pdf)

2. http://www.nobelprize.org/nobel_prizes/medicine/laureates/1998/

3. Griffith, T. M., Lewis, M. J., Newby, A. C., Henderson, A. H. J. Amer. Coll. Cardiology, 1988, 12, 797-806.

4. Ignarro, L. J. Hypertension, 1990, 16, 477-483.

5. (a) Tuteja, N.; Chandra, M.; Tuteja, R.; Misra, M. J. Biomed. Biotechnol., 2004, 4, 227-237; (b) Dredt, D. S. W.; Snyder, S. H. Annu. Rev. Biochem. 1994, 63, 175-195. (c) Calabrese, V.; Bates, T. E.; Stella, A. M. G. Neurochem. Res., 2000, 25, 1315-1341;

6. Wink, D.A.; Vodovotz, Y.; Laval, J.; Laval, F.; Dewhirst, M. W.; Mitchell, J. B. Carcinogenesis, 1998, 19, 711-721.

7. Davies, S.A., Stewart, E.J., Huesmaan, G. R. and Skaer, N. J. Am. J. Physiol., 1997, 273, R823-827.

8. Hou, Y. C.; Janczuk, A.; Wang, P. G. Curr. Pharm. Des. 1999, 5, 417-471. PMID 10390607.

9. (a) Radicals for life: The various forms of nitric oxide. E. van Faassen and A. Vanin, eds. Elsevier, Amsterdam 2007. ISBN 978-0-444-52236-8; (b) van Faassen, E. E.; Bahrami, S.; Feelisch, M.; Hogg, N.; , M.; Kim-Shapiro, D. B.; Kozlov, A. V.; Li, H.; Lundberg, J. O.; Mason, R.; Nohl, H.; Rassaf, T.; Samouilov, A.; Slama-Schwok, A.; Shiva, S.; Vanin A. F.; Weitzberg, E.; Zweier, J.; Gladwin, M. T. Med. Res. Rev., 2009, 29, 683-741.

10. (a) Tanaka, Y.; Tang, G.; Takizawa, K.; Otsuka, K.; Eghbali, M.; Song, M.; Nishimaru, K.; Shigenobu, K.; Koike, K.; Stefani, E.; Toro, L. J. Pharmacol. Exptal. Therapeutics, 2005, 317, 341-354; (b) Yoon, Y.; Song, U.; Hong, S. H.; Kim, J. Q. Clinic. Chem. 2000, 46, 1626-1630.

11. Green, S. J. Nature Medicine, 1995, 1, 515-517.

12. (a) Green, S. J.; Crawford, R. M.; Hockmeyer, J. T.; Meltzer, M. S.; Nacy, C. A. J. Immunol. 1990, 145, 4290-7; (b) Seguin, M. C.; Klotz, F. W.; Schneider, I.; Weir, J. P.; Goodbary, M.; Slayter, M; Raney, J. J.; Aniagolu, J. U.; Green, S. J. J. Exptal. Medicine, 1994, 180, 353-8; (c) Mellouk, S.; Green, S. J.; Nacy, C. A.; Hoffman, S. L. J. Immunol., 1991, 146, 3971-6; (d) Klotz, F. W.; Scheller, L. F.; Seguin, M. C.; Kumar, N.; Marletta, M. A.; Green, S. J.; Azad, A. F. J. Immunol., 1995, 154, 3391-5.

13. Shaw, A. W.; Vosper, A. J. J. Chem. Soc., Faraday Trans. 1977, 1239.

14. (a) Pogrebnaya, V. L.; Usov, A. P.; Baranov, A. V.; Nesterenko, A. I.; Bez'yazychnyi, P. I. J. Appl. Chem. USSR. 1987, 48, 1004. (b) Pires, M.; Rossi, 
M. J.; Ross, D. S. Int. J. Chem. Kinet. 1994, 26, 1207. (c) Goldstein, S.; Czapski, G. J. Am. Chem. Soc. 1995, 117, 12078.

15. Liu, X.; Miller, M. J. S.; Joshi, M. S.; Thomas, D. D.; Lancaster, J. R., Jr. Proc. Natl. Acad. Sci. U.S.A. 1998, 95, 2175.

16. (a) Moncada, S.; Palmer, R. M. J.; Higgs, E. A. Pharmacol. Rev., 1991, 43, 109142; (b) Furchgot, R.F.; Vanhoutte, P.M. FASEB J,. 1989, 3, 2007; (c) Lancaster Jr., J.R., Nitric Oxide Biology and Pathology, ed. Ignarro, L.J., Academic Press, San Diego, 2000.

17. (a) Kojima, H., Nagano, T. Chem. Rev., 2002, 102, 1235-1269; (b) Yang, Y., Zhao, Q., Wei Feng, W., Li, F. Chem. Rev., 2013, 113, 192 -270.

18. (a) Moncada, S.; Palmer, R. M.; Higgs, E. A. Pharmacol. Rev. 1991, 43, 109. (b) Conner, E. M.; Grisham, M. B. Methods, 1995, 7, 3.

19. (a) Palmer, R. M. J.; Ferrige, A. G.; Moncada, S. Nature 1987, 327, 524; (b) Hampl, V.; Walters, C.; Archer, S. L. In Methods in Nitric Oxide Research; Feelisch, M., Stamler, J. S., Eds.; John Wiley \& Sons: New York, 1996; p 309.

20. (a) Leone, A. M.; Furst, V. W.; Foxwell, N. A.; Cellek, S.; Moncada, S. Biochem. Biophys. Res. Commun. 1996, 221, 37; (b) Kikuchi, K.; Nagano, T.; Hayakawa, H.; Hirata, Y.; Hirobe, M. J. Biol. Chem. 1993, 268, 23106.

21. Malinski, T.; Mesaros, S.; Tomboulian, P. Methods Enzymol. 1996, 268, 58.

22. (a) Henry, Y.; Ducrocq, C.; Drapier, J. C.; Servent, D.; Pellat, C.; Guissani, A. Eur. Biophys. J. 1991, 20, 1; (b) Henry, Y.; Lepoivre, M.; Drapier, J. C.; Ducrocq, C.; Boucher, J.-L.; Guissani, A. FASEB J. 1993, 7, 1124; (c) Wilcox, D. E.; Smith, R. P. Methods: A Companion to Methods in Enzymology 1995, 7, 59; (d) Rochelle, L. G.; Kruszyna, H.; Smith, R. P. Methods Neurosci. 1996, 31, 3; (e) Yoshimura, T.; Yokoyama, H.; Fujii, S. J. Magn. Reson. Anal. 1997, 3, 125; (f) Nitric Oxide Research from Chemistry to Biology: EPR Spectroscopy of Nitrosylated Compounds; Henry, Y. A., Guissani, A., Ducastel. B., Eds.; Springer: New York, 1997; (g) Nitric Oxide in Transplant Rejection and Antitumor Defence; Lukiewicz, S., Zweir, J. L., Eds.; Kluwer Academic Publishers: Boston, 1998; (h) Pou, S.; Halpern, H. J.; Tsai, P.; Rosen, G. M. Acc. Chem. Res. 1999, 32, 155; (i) Fujii, H.; Kazama, S.; Berliner, L. J. Curr. Top. Biophys. 1999, 23, 11; (j) Henry, Y.; Guissani, A. Analusis 2000, 28, 445; (k) Fujii, S.; Yoshimura, T. Antioxid. Redox Signaling 2000, 2, 879; (l) Berliner, L. J.; Khramtsov, V.; Fujii, H.; Clanton, T. L. Free Radical Biol. Med. 2001, 30, 489.

23. (a) Arroyo, C. M.; Kohno, M. Free Radical Res. Commun. 1991, 14, 145; (b) Pronai, L.; Ichimori, K.; Nozaki, H.; Nakazawa, H.; Okino, H.; Carmichael, A. J.; Arroyo, C. M. Eur. J. Biochem. 1991, 202, 923; (c) Arroyo, C. M.; Forray, C. Eur. I. Pharmacol. 1991, 208, 157. 
24. (a) Henry, Y.; Ducrocq, C.; Drapier, J. C.; Servent, D.; Pellat, C.; Guissani, A.

Eur. Biophys. J. 1991, 20, 1; (b) Henry, Y.; Lepoivre, M.; Drapier, J. C.; Ducrocq, C.; Boucher, J.-L.; Guissani, A. FASEB J. 1993, 7, 1124; (c) Nitric Oxide Research from Chemistry to Biology: EPR Spectroscopy of Nitrosylated Compounds; Henry, Y. A., Guissani, A., Ducastel. B., Eds.; Springer: New York, 1997; (d) Cheng, L.; Richter-Addo, G. B. In The Porphyrin Handbook; Kadish, K. M., Smith, K. M., Guilard, R., Eds.; Academic Press: San Diego, 2000; Vol. 4, p 219; (e) Yoshimura, T. In Bioradicals Detected by ESR Spectroscopy; OhyaNishiguchi, H., Packer, L., Eds.; Birkha“user Verlag: Basel, 1995, 217.

25. (a) Osiecki, J. H.; Ullman, E. J. Am. Chem. Soc. 1968, 90, 1078; (b) Ullman, E. F.; Call, L.; Osiecki, J. H. J. Org. Chem. 1970, 35, 3623; (c) Ullman, E. F.; Osiecki, J. H.; Boocock, D. G. B.; Darcy, R. J. Am. Chem. Soc. 1972, 94, 7049.

26. (a) Yoshimura, T.; Yokoyama, H.; Fujii, S.; Takayama, F.; Oikawa, K.; Kamada, H. Nat. Biotechnol. 1996, 14, 992; (b) Huie, R. E.; Padmaja, S. Free Radical Res. Comms. 1993, 18, 195.

27. (a) Thorp-Greenwood, F. Organometallics, 2012, 31, 5686-5692; (b) Balasingham, R. G.; Coogan, M. P.; Thorp-Greenwood, F. L. Dalton Trans. 2011, 40, 11663; (c) Zhao, Q.; Huang, C. H.; Li, F. Y. Chem. Soc. Rev. 2011, 40, 2508;

(c) Fernández-Moreira, V.; Thorp-Greenwood, F. L.; Coogan, M. P. Chem. Commun. 2010, 46, 186.

28. (a) Nagano, T.; Yoshimura, T. Chem. Rev. 2002, 102, 1235. (b) Yang, Y.; Zhao, Q.; Feng, F.; Li, F. Chem. Rev. 2013, 113, 192-270.

29. Kojima, H.; Sakurai, K.; Kikuchi, K.; Kawahara, S.; Kirino, Y.; Nagoshi, H.; Hirata, Y.; Akaike, T.; Maeda, H.; Nagano, T. Biol. Pharm. Bull. 1997, 20, 12291232.

30. (a) Suzuki, N.; Kojima, H.; Urano, Y.; Kikuchi, K.; Hirata, Y.; Nagano, T. J. Biol. Chem. 2002, 277, 47. (b) Kojima, H.; Nakatsubo, N.; Kikuchi, K.; Kawahara, S.; Kirino, Y.; Nagoshi, H.; Hirata, Y.; Nagano, T. Anal. Chem. 1998, 70, 2446. (c) Itoh, Y.; Ma, F. H.; Hoshi, H.; Oka, M.; Noda, K.; Ukai, Y.; Kojima, H.; Nagano, T.; Toda, N. Anal. Biochem. 2000, 287, 203; (d) Yu, C.; Wu, Y.; Zeng, F.; Wu, S. Journal of Materials Chemistry B: Materials for Biology and Medicine. 2013, 1, 4152-4159

31. (a) Gabe, Y.; Urano, Y.; Kikuchi, K.; Kojima, H.; Nagano, T. J. Am.Chem. Soc. 2004, 126, 3357; (b) Gabe, Y.; Ueno, T.; Urano, Y.; Kojima, H.; Nagano, T. Anal. Bioanal. Chem. 2006, 386, 621.

32. Hu, J. X.; Yin, L. L.; Xu, K. H.; Gao, J. J.; Tong, L. L.; Tang, B. Anal. Chim. Acta, 2008, 606, 57.

33. (a) Heiduschka, P.; Thanos, S. Neuroreport, 1998, 9, 4051. (b) Galindo, F.; Kabir, N.; Gavrilovic, J.; Russell, D. A. Photochem. Photobio. Sci, 2008, 7, 126.

34. Sasaki, E.; Kojima, H.; Nishimatsu, H.; Urano, Y.; Kikuchi, K.; Hirata, Y.; Nagano, T. J. Am. Chem. Soc. 2005, 127, 3684. 
35. (a) Zhang, R.; Ye, Z. Q.; Wang, G. L.; Zhang, W. Z.; Yuan, J. L. Chem. Eur. J. 2010, 16, 6884; (b) Choi, A. W. T.; Poon, C. S.; Liu, H. W.; Cheng, H. K.; Lo, K. New J. Chem., 2013, 37, 1711-1719.

36. Nagano, T.; Takizawa, H.; Hirobe, M. Tetrahedron Lett. 1995, 36, 8239.

37. (a) Nakatsubo, N.; Kojima, H.; Kikuchi, K.; Nagoshi, H.; Hirata, Y.; Maeda, D.; Imai, Y.; Irimura, T.; Nagano, T. FEBS Lett. 1998, 427, 263; (b) Kojima, H.; Sakurai, K.; Kikuchi, K.; Kawahara, S.; Kirino, Y.; Nagoshi, H.; Hirata, Y.; Nagano, T. Chem. Pharm. Bull. 1998, 46, 373.

38. Nakatsubo, N.; Kojima, H.; Sakurai, K.; Kikuchi, K.; Nagoshi, H.; Hirata, Y.; Akaike, T.; Maeda, H.; Urano, Y.; Higuchi, T.; Nagano, T. Biol. Pharm. Bull. 1998, 21, 1247.

39. (a) Sun, W. C.; Gee, K. R.; Klaubert, d. H.; Haugland, R. P. J. Org. Chem. 1997, 62, 6469; (b) Tanaka, K.; Miura, T.; Umezawa, N.; Urano, Y.; Kikuchi, K.; Higuchi, T.; Nagano, T. J. Am. Chem. Soc. 2001, 123, 2530-2536; (c) Yamaguchi, K.; Tamura, Z.; Maeda, M. Acta Crystallogr. 1997, C53, 284; (d) Namin, S. M.; Nofallah, S.; Joshi, M. S.; Kavallieratos, K.; Tsoukias, N. M. "Kinetic analysis of DAF-FM activation by NO: Toward calibration of a NOsensitive fluorescent dye”, Nitric Oxide, Biology and Chemistry, 2013, 28, 39-46.

40. Yang, Y. J.; Seidlits, S. K.; Adams, M. M.; Lynch, V. M.; Schmidt, C. E.; Anslyn, E. V.; Shear, J. B. J. Am. Chem. Soc. 2010, 132, 13114.

41. (a) Zheng, H.; Shang, G. Q.; Yang, S. Y.; Gao, X.; Xu, J. G. Org. Lett. 2008, 10, 2357. (b) Sun, C. D.; Shi, W.; Song, Y. C.; Chen, W.; Ma, H. M. Chem. Commun. 2011, 47, 8638; (c) Yuan, L.; Lin, W.; Xie, Y.; Chen, Bin; Song, J. Chem. Commun. 2011, 47, 9372-9374.

42. Hilderbrand, S. A.; Lippard, S. J. Inorg. Chem. 2004, 43, 4674; Franz, K. J.; Singh, N.; Spingler, B.; Lippard, S. J. Inorg. Chem. 2000, 39, 4081.

43. Hilderbrand, S. A.; Lim, M. H.; Lippard, S. J. J. Am. Chem. Soc. 2004, 126, 4972.

44. Lim, M. H.; Lippard, S. J. Inorg. Chem. 2004, 43, 6366.

45. Victor, E., Kim, S., Lippard, S. Inorg.Chem. 2014, 53, 12809-1221.

46. (a) Tsuge, K.; DeRosa, F.; Lim, M. D.; Ford, P. C. J. Am. Chem.Soc. 2004, 126, 6564; (b) Lim, M. H.; Lippard, S. J. J. Am. Chem. Soc. 2005, 127, 12170.

47. (a) Lim, M. H.; Wong, B. A.; Pitcock, W. H.; Mokshagundam, D.; Baik, M. H.; Lippard, S. J. J. Am. Chem. Soc. 2006, 128, 14364; (b) McQuade, L. E.; Lippard, S. J. Inorg. Chem. 2010, 49, 7464; (c) McQuade, L. E.; Ma, J.; Lowe, G.; Ghatpande, A.; Gelperin, A.; Lippard, S. J. Proc. Natl. Acad. Sci. U.S.A. 2010, 107, 8525; (d) Pluth, M. D.; Chan, M. R.; McQuade, L. E.; Lippard, S. J. Inorg. Chem. 2011, 50, 9385.

48. Pluth, M. D.; McQuade, L. E.; Lippard, S. J. Org. Lett. 2010, 12, 2318.

49. Lim, M. H.; Xu, D.; Lippard, S. J. Nat. Chem. Biol. 2006, 2, 375. 
50. Hu, X. Y.; Wang, J.; Zhu, X.; Dong, D. P.; Zhang, X. L.; Wu, S. O.; Duan, C. Y. Chem. Commun. 2011, 47, 11507.

51. Haas, K. L.; Franz, K.J. Application of Metal Coordination Chemistry to explore and Manipulate Cell Biology. Chem. Rev., 2009, 109, 4921-4960.

52. (a) Frau`sto da Silva, J. J. R.; Williams, R. J. P. The Biological Chemistry of the Elements, 2nd ed.; Oxford University Press: Oxford, U.K., 2001; (b) Lippard, S. J.; Berg, J. M. Principles of Bioinorganic Chemistry; University Science Books: Mill Valley, CA, 1994. (c) Mann, S. In Biological Inorganic Chemistry: Structure and ReactiVity; Bertini, I., Gray, H. B., Stiefel, E. I., Valentine, J. S., Eds.; University Science Books: Sausalito, CA, 2007; 79. (d) Evenas, J.; Malmendal, A.; Forsen, S. Curr. Opin. Chem. Biol. 1998, 2, 293; (e) Pyle, A. J. Biol. Inorg. Chem. 2002, 7, 679; (f) Vinod, K.; Misra, D. E. D. Biopolymers 1998, 48, 113; (g) Winge, D. R. In Biological Inorganic Chemistry: Structure and ReactiVity; Bertini, I., Gray, H. B., Stiefel, E. I., Valentine, J. S., Eds.; University Science Books: Sausalito, CA, 2007; p 613; (h) Frederickson, C. J.; Koh, J.-Y.; Bush, A. I. Nat. ReV. Neurosci. 2005, 6, 449. (i) Chen, P. R.; He, C. Curr. Opin. Chem. Biol. 2008, 12, 214; (j) Pennella, M. A.; Giedroc, D. P. BioMetals 2005, 18, 413.

53. Eroy-Reveles, A. A.; Leung, Y.; Beavers, C. M.; Olmstead, M. M.; Mascharak, P. K. J. Am. Chem. Soc. 2008, 130, 4447.

54. (a) Cai, W.; Niu, G.; and Chen, X. Eur. J. Nucl. Med. Mol. Imaging, 2008, 35, 186-208. (b) Deutsch, E.; Libson, K.; Vanderheyden, J.-L.; Ketring, A. R.; Maxon, H. R Nucl. Med. Biol. 1986, 13, 465-477. (c) Dilworth, J.; Parrot, S. Chem. Soc. Rev. 1998, 27, 43-55; (d) Arteaga, C. Semin. Oncol., 2003, 30/7, 314; (b) Ritter, C. A.; Arteaga, C. Semin. Oncol., 2003, 30/1, 3-11; (c) Mass, R. D. Int. J. Radiat. Oncol., Biol., Phys., 2004, 58, 932-940; (e) Albanell, J.; Gasc'on, P. Curr. Drug Targets, 2005, 6, 259-274; (f) Vallbohmer, D.; Lenz,H. J. Clin. Colorectal Cancer, 2005, 5, S19-S27.

55. Fernandes, C.; Santos, I. C.; Santos, I.; Pietzsch, H. J.; Kunstler, J. U.; Kraus, W.; Rey, A.; Margaritis, N.; Bourkoula, A.; Chiotellis, A. Dalton Trans., 2008, 24, 3215-3225.

56. (a) Johnstr“om, P.; Fredriksson, A.; Thorell, J. O.; Stone-Elander, S. J. Labelled Compd. Radiopharm., 1998, 41, 623-629; (b)Ben-David, I.; Rozen, Y.; Ortu, G.; Mishani, E. Appl. Radiat. Isot., 2003, 58, 209-217; (c) Mishani, E.; Abourbeh, G.; Rozen, Y.; Jacobson, O.; Laky, D.; Ben, I.; Levitzki, A.; Shaul, M. Nucl. Med. Biol., 2004, 31, 469-476.

57. (a) Fredriksson, A.; Johnstr“om, P.; Thorell, J. O.; von Heijne, G.; Hassan, M; Eksborg, S.; Kogner, P.; Borgst "om, P.; Ingvar, M.; Stone-Elander, S. Life Sci., 1999, 65, 165-174; (b) Wang, J. Q.; Gao, M.; Miller, K. D.; Sledge, G. W.; Zheng, Q. H. Bioorg. Med. Chem. Lett., 2006, 16, 4102-4106.

58. (a) Bonasera, T.; Ortu, G.; Rozen, Y.; Krais, R.; Freedman, N. M.; Chisin, R.; Gazit, A.; Levitzki, A.; Mishani, Nucl. Med. Biol., 2001, 28, 359-374; (b) Seimbille, Y.; Phelps, M. E.; Czernin, J.; Silverman, D. H. S.; J. LabelledCompd. 
Radiopharm., 2005, 48, 829-843; (c) Dissoki, S.; Aviv, Y.; Laky, D.; Abourbeh, G.; Levitzki A.; Mishiani, E. Appl. Radiat. Isot., 2007, 65, 1140-1151.

59. Johnsson, N.; Johnsson, K. ACS Chem. Biol. 2007, 2, 31.

60. (a) Beeby, A.; Botchway, S. W.; Clarkson, I. M.; Faulkner, S.; Parker, A. W.; Parker, D.; Williams, J. A. G. J. Photochem. Photobiol., B. 2000, 57, 83. (b) Vereb, G.; Jares-Erijman, E.; Selvin, P. R; Jovin, T. M. Biophys. J. 1998, 74, 2210.

61. (a) Manning, H. C.; Goebel, T.; Thompson, R. C.; Price, R. R.; Lee, H.; Bornhop, D. J. Bioconjugate Chem. 2004, 15, 1488; (b) Lo, K. K. W.; Lee, T. K. M.; Lau, J. S. Y.; Poon, W. L.; Cheng, S. H. Inorg. Chem. 2008, 47, 200; (c) Lo, K. K. W.; Louie, M. W.; Sze, K. S.; Lau, J. S. Y. Inorg. Chem. 2008, 47, 602; (d)

Neugebauer, U.; Pellegrin, Y.; Devocelle, M.; Forster, R. J.; Signac, W.; Moran, N.; Keyes, T. E. Chem. Commun. 2008, 42, 5307; (e) Petoud, S.; Muller, G.; Moore, E. G.; Xu, J.; Sokolnicki, J.; Riehl, J. P.; Le, U. N.; Cohen, S. M.; Raymond, K. N. J. Am. Chem. Soc. 2007, 129, 77; (f) Moore, E. G.; Samuel, A. P. S.; Raymond, K. N. Acc. Chem. Res. 2009, 42, 542.

62. (a) Yu, M.; Zhao, Q.; Shi, L.; Li, F.; Zhou, Z.; Yang, H.; Yi, T.; Huang, C. Chem. Commun. 2008, 18, 2115; (b) Ma, D.-L.; Wong, W.-L.; Chung, W.-H.; Chan, F.Y.; So, P.-K.; Lai, T.-S.; Zhou, Z.-Y.; Leung, Y.-C.; Wong, K.-Y. Angew. Chem., Int. Ed. 2008, 47, 3735.

63. (a) Botchway, S. W.; Charnley, M.; Haycock, J. W.; Parker, A. W.; Rochester, D. L.; Weinstein, J. A.; Williams, J. A. G. Proc. Natl. Acad. Sci. U.S.A. 2008, 105, 16071; (b) Weissleder, R. Nat. Biotechnol. 2001, 19, 316; (c) Williams, J. A. G. Top. Curr. Chem. 2007, 281, 205; (d) McMillin, D. R.; Moore, J. J. Coord. Chem. ReV. 2002, 229, 113; (e) Koo, C. K.; Wong, K. L.; Man, C. W. Y.; Lam, Y. W.; So, K. Y.; Tam, H. L.; Tsao, S. W.; Cheah, K. W.; Lau, K. C.; Yang, Y. Y.; Chen, J. C.; Lam, M. H. W. Inorg. Chem. 2009, 48, 872; (f) Furuta, T.; Wang, S. S. H.; Dantzker, J. L.; Dore, T. M.; Bybee, W. J.; Callaway, E. M.; Denk, W.; Tsien, R. Y. Proc. Natl. Acad. Sci. U.S.A. 1999, 96, 1193; (g) Wu, P.; Wong, E. L.-M.; Ma, D.-L.; Tong, G. S.-M.; Ng, K.-M.; Che, C.-M. Chem. Eur. J. 2009, 15, 3652.

64. (a) Chauvin, A. S.; Comby, S.; Song, B.; Vandevyver, C. D. B.; Thomas, F.; Bunzli, J. C. G. Chem. Eur. J. 2007, 13, 9515; (b) Deiters, E.; Song, B.; Chauvin, A. S.; Vandevyver, C. D. B.; Bunzli, J. C. G. New J. Chem. 2008, 32, 1140; (c) Chauvin, A. S.; Comby, S.; Song, B.; Vandevyver, C. D. B.; Bunzli, J. C. G. Chem. Eur. J. 2008, 14, 1726; (d) Elhabiri, M.; Scopelliti, R.; Bunzli, J. C. G.; Piguet, C. J. Am. Chem. Soc. 1999, 121, 10747.

65. Law, G. L.; Wong, K. L.; Man, C. W. Y.; Wong, W. T.; Tsao, S. W.; Lam, M. H. W.; Lam, P. K. S. J. Am. Chem. Soc. 2008, 130, 3714.

66. Napoli, C.; Ignarro, L. J. Annu. ReV. Pharmacol. Toxicol. 2003, 43, 97.

67. Pavlos, C. M.; Xu, H.; Toscano, J. P. Curr. Top. Med. Chem. 2005, 5, 635.

68. Rose, M. J.; Mascharak, P. K. Coord. Chem. Rev. 2008, 252, 2093. 
69. Afshar, R. K.; Patra, A. K.; Olmstead, M. M.; Mascharak, P. K. Inorg.Chem. 2004, 43, 5736.

70. Patra, A. K.; Mascharak, P. K. Inorg. Chem. 2003, 42, 7363.

71. Rose, M. J.; Mascharak, P. K. Chem. Commun. 2008, 33, 3933.

72. (a) Greene, S. N.; Richards, N. G. J. Inorg. Chem. 2004, 43, 7030; (b) Works, C. F.; Jocher, C. J.; Bart, G. D.; Bu, X.; Ford, P. C. Inorg.Chem. 2002, 41, 3728.

73. Stufkens, D. J.; Vlcek, A. Coord. Chem. Rev. 1998, 177, 127.

74. (a) Amoroso, A. J.; Coogan, M. P.; Dunne, J. E.; Fernandez-Moreira, V.; Hess, J. B.; Hayes, A. J.; Lloyd, D.; Millet, C.; Pope, S. J. A.; Williams, C. Chem. Commun. 2007, 29, 3066; (b) Amoroso, A. J.; Arthur, R. J.; Coogan, M. P.; Court, J. B.; Fernandez-Moreira, V.; Hayes, A. J.; Lloyd, D.; Millet, C.; Pope, S. J. A. New J. Chem. 2008, 32, 1097.

75. (a) Shaul, M.; Abourbeh, G.; Jacobson, O.; Rozen, Y.; Desideriu, L.; Levitzki, A.; Mishani, E. Bioorg. Med. Chem., 2004, 12, 3421-3429; (b) Pal, A.; Glekas, A.; Doubrovin, M.; Balatoni, J.; Beresten, T.; Maxwell, D.; Soghomonyan, S.; Shavrin, A.; Ageyeva, L.; Finn, R.; Larson, S. M.; Bornmann, W.; Gelovani, J. G. Mol. Imaging Biol., 2006, 8, 262-277.

76. Rossenaar, B.D.; Stufkens, D. J.; Vlcek, A. Inorg. Chem. 1996, 35, 2902.

77. Knor, G.; Leirer, M.; Vogler, A. Inorg. Chim. Acta, 1999, 288, 150.

78. Ringertz, N. Alfred Nobel's health and his interest in medicine; The Nobel Foundation, 1998. http://www.nobel.se/nobel/alfrednobel/biographical/ringertz/.

79. Al-Sa’doni, H.; Ferro, A. Clin. Sci. 2000, 98, 507.

80. (a) Wrighton, M.; Morse, D. L. J. Am. Chem. Soc. 1974, 96, 998; (b) Luong, J.C. ; Nadjo, L.; Wrighton, M.S. J. Am. Chem. Soc. 1978, 100, 5790; (c) Morse, D.L.; Wrighton, M.S. J. Am. Chem. Soc. 1976, 98, 3931; (d) L.W. Houk, G.R. Dobson, Inorg. Chem. 1966, 5, 2119; (e) G.J. Stor, S.L. Morrison, D.J. Stufkens, A. Oskam, Organometallics 1994, 213, 2641.

81. Janero, D. R. Free Radical Biol. Med. 2000, 28, 1495; (b) Yamamoto, T.; Bing, R. J. Proc. Soc. Exp. Biol. Med. 2000, 225, 200; (c) Lehmann, J. Exp. Opin. Ther. Pat. 2000, 10, 559; (d) Feelisch, M. Arch. Pharmacol. 1998, 358, 113; (e) Megson, I. L. Drugs Future 2000, 25, 701.

82. Wang, P. G.; Xian, M.; Tang, X.; Wu, X.; Wen, Z.; Cai, T.; Janczuk, A. J. Chem. Rev. 2002, 102, 1091-1134.

83. Baker, J. W.; Heggs, T. G. Chem. Ind. 1954, 464.

84. (a) Honeyman, J.; Morgan, J. W. Adv. Carbohydr. Chem. 1957, 12, 117; (b) McMillen, D. F.; Golden, D. M. Annu. Rev. Phys. Chem. 1982, 33, 493; (c) Capellos, C.; Fisco, W. J.; Ribaudo, C.; Hogan, V. D.; Campisi, F. X.; Murphy, F. X.; Castorina, T. C.; Rosenblatt, D. H. Int. J.Chem. Kinet. 1984, 16, 1009; (d) Baker, J. W.; Easty, D. M. J. Chem. Soc. 1952, 1208. 
85. Needleman, P.; Johnson, E. M. J. Pharmacol. Exp. Ther. 1973, 184, 709.

86. Williams, D. L. H. Nitrosation; Cambridge University Press: Cambridge; 150172.

87. Doyle, M. P.; Terpstra, J. W.; Pickering, R. A.; Le Poire, D. M. J. Org. Chem. 1983, 48, 3379.

88. (a) Grossi, L.; Strazzar, S. J. Org. Chem. 1999, 64, 8076. (b) Garcia-Santos, P.; Calle, E.; Gonzalez-Mancebo, S.; Casado, J. Monatsh. Chem. 1996, 127, 997. (c) McMillen, D. F.; Golden, D. M. Annu. Phys. Chem. 1982, 33, 493.

89. Pechmann, H. V. Chem. Ber. 1895, 28, 855.

90. (a) Magee, P. N.; Barnes, J. M. Br. J. Cancer 1956, 10, 114; (b) Magee, P. N.; Hultin, T. Biochem. J. 1962, 83, 106; (c) Magee, P. N.; Farber, E. Biochem. J. 1962, 83, 114; (d) Lijinsky, W. Chemistry and Biology of N-nitroso Compounds; Cambridge University Press: Cambridge, 1992.

91. (a) Keefer, L. K.; Wink, D. A.; Dunams, T. M.; Hrabie, J. A. U.S. Patent 5,212,204, 1993; Chem. Abstr. 1990, 113, 145344; (b) Diodati, J. G.; Keefer, L. K. U.S. Patent 5,185,376, 1993; Chem. Abstr. 1993, 118, 94326.

92. Castagnou, R.; Pabia, J. Bull. Soc. Pharm. Bordeaux 1953, 91, 48.

93. (a) Alston, T. A.; Porter, D. J. T.; Bright, H. J. J. Biol. Chem. 1985, 260, 4069; (b) Lawless, J. G.; Hawley, M. D. Anal. Chem. 1968, 40, 948.

94. (a) Balaban, A. T.; Negoita, N.; Pascaru, I. Rev. Roum. Chim. 1971, 16, 721; (b) Vega, J. M.; Garrett, R. H.; Siegel, L. M. J. Biol. Chem. 1975, 250, 7980.

95. (a) Rehse, K.; Birkhofer, G. Arch. Pharm. (Weinheim) 1995, 328, 77; (b) Rehse, K.; Ludtke, E. Arch. Pharm. (Weinheim) 1995, 328, 17; (c) Rehse, K.; Ciborski, T.; Ludtke, E. Arch. Pharm. (Weinheim)1994, 327, 771; (d) Rehse, K.; Ludtke, E. Arch. Pharm. (Weinheim) 1994, 327, 581; (e) Rehse, K.; Schleifer, K. J.; Martens, A.; Kampfe, M. Arch. Pharm. (Weinheim) 1994, 327, 393; (f) Rehse, K.; Schleifer, K. J. Arch. Pharm. (Weinheim) 1993, 326, 929; (g) Rehse, K.; Schleifer, K. J.; Ciborski, T.; Bohn, H. Arch. Pharm.(Weinheim) 1993, 326, 791; (h) Rehse, K.; Kampfe, M.; Schleifer, K. J. Arch. Pharm. (Weinheim)1993, 326, 483. (i) Rehse, K.; Brummer, U.; Unsold, E. Pharmacology 1998, 53, 820.

96. Tasker, H. S.; Jones, H. O. J. Chem. Soc. 1909, 95, 1910.

97. DeMaster, E. G.; Quast, B. J.; Redfern, B.; Nagasawa, H. T. Biochemistry 1995, 34, 11494.

98. Szacilowski, K.; Stasicka, Z. Prog. React. Kinet. Mech. 2000, 26, 1.

99. Piloty, O. Ber. 1898, 31, 1878.

100. Pou, S.; Anderson, D. E.; Surichamon, W.; Keaton, L. L.; Tod, M. L. Mol. Pharmacol. 1994, 46, 709. 
101. Stilo, A.; Medana, C.; Ferrarotti, B.; Gasco, A. L.; Ghigo, D.; Bosia, A.; Martorana, P. A.; Gasco, A. Pharmacol. Res. 2000, 41, 469.

102. Singh, P.; Boocock, D. G. B.; Ullman, E. F. Tetrahedron Lett. 1971, 42, 3935.

103. (a) Severina, I. S.; Ryaposova, I. K.; Volodarsky, L. B.; Mazhuhin, D. C.; Tichonov, A. Ya.; Schwartz, G. Ya.; Granik, V. G.; Grigorgyev, N. B.; Grigorgyev, N. B. Biochem. Mol. Biol. Int. 1993, 30, 357; (b) Belushkina, N. N.; Grigoryev, N. B.; Severina, I. S. Biokhimiya 1994, 59, 1689; (c) Severina, I. S.; Belushkina, N. N.; Grigoryev, N. B. Biochem. Mol. Biol. Int. 1994, 33, 957; (d) Shvarts, G. Ya.; Grigoryev, N. B.; Severina, I. S.; Ryaposova, I. K.; Laptiskaya, A. S.; Volodarsky, L. B.; Tikhonov, A. Ya.; Kurbnikova, I. F.; Mazhukin, D. G.; Granik, V. G. Khim.-Farm. Zh. 1994, 28, 38; (e) Ryaposova, I. K.; Grigoryev, N. B.; Severina, I. S. Inst. Biomed. Chem. 1994, 59, 537.

104. Utepbergenov, D. I.; Khramtsov, V. V.; Vlassenko, L. P.; Markel, A. L.; Mazhukin, D. G.; Tiknonov, A. Ya.; Volodarsky, L. B. Biochem. Biophys. Res. Commun. 1995, 214, 1023.

105. (a) Ghosh, P. B. J. Chem. Soc. B 1968, 334; (b) Ghosh, P. B.; Whitehouse, M. W. J. Med. Chem. 1968, 11, 305; (c) Ghosh, P. B.; Whitehouse, M. W. J. Med. Chem. 1969, 12, 505.

106. (a) Feelisch, M.; Schonafinger, K.; Noack, E. Biochem. Pharmocol. 1992, 44, 1149; (b) Medana, C.; Ermondi, G.; Fruttero, R.; Di Stilo, A.; Ferretti, C. Gasco, A. J. Med. Chem. 1994, 37, 4412.

107. Sako, M.; Oda, S.; Ohara, S.; Hirota, K.; Maki, Y. J. Org. Chem. 1998, 63, 6947.

108. Finnegan, W. G.; Henry, R. A. J. Org. Chem. 1965, 30, 567.

109. Masuda, K.; Kamiya, T. Japan Patent JP 45020904, 1974.

110. Masuda, K.; Kamiya, T.; Kashiwa, K. Chem. Pharm. Bull. 1971, 19, 559.

111. (a) Dendorfer, A. HERZ 1996, 21 (Suppl. 1), 38; (b) Schonafinger, K. II Farmaco 1999, 54, 316; (c) Newton, C. G.; Ramsden, C. A. Tetrahedron 1982, 38, 2965.

112. (a) Brookes, P.; Walker, J. J. Chem. Soc. 1957, 4409; (b) Kato, H.; Hashimoto, M.; Ohta, M. Nippon Kagaku Zasshi 1957, 78, 707.

113. (a) Fellisch, M.; Ostrowski, J.; Naock, E. J. Cardiovasc. Pharmacol. 1989, 14 (Suppl. 11), S13; (b) Noack, E.; Feeelisch, M. J. Cardiovasc. Pharmacol. 1989, 14 (Suppl. 11), S1.

114. Thomas, G.; Ramwell, P. W. Biochem. Biophys. Chem. Commun. 1989, 164, 889.

115. (a) Hino, M.; Takase, S.; Itoh, Y.; Uchida, I.; Okamoto, M.; Hashimoto, M.; Kohsaka, M. Chem. Pharm. Bull. 1989, 37, 2864; (b) Arita, M.; Morita, K.; Sato, H.; Uehata, M. JP Patent 4364157, 1992.

116. Decout, J. L.; Roy, B.; Tontecave, M.; Muller, J. C.; Williams, P.H.; Loyaux, D. Bioorg. Med. Chem. Lett. 1995, 5, 973. 
117. (a) Gross, P. Crit. Rev. Toxicol. 1985, 14, 87; (b) Chang, C. P.; Pan, S. P.; Lin, M. T. Br. J. Pharm. 2001, 132, 1524; (c) Lin, M. T.; Pan, S. P.; Lin, J. H.; Yang, Y. L. Br. J. Pharm. 1999, 127, 1511; (d) Santoian, E. C.; Thomas, G.; Angerio, A. D.; Kot, P. A.; Ramwell, P. W. Angiology 1993, 44, 897.

118. (a) Nicholls, P. Biochem. J. 1964, 90, 331; (b) Craven, P. A.; DeRubertis, F. R.; Pratt, D. W. J. Biol. Chem. 1979, 251, 8213.

119. (a) Cory, J. G.; Carter, G. L.; Bacon, P. E.; Tang, A.; Lien, E. J. Biochem. Pharmacol. 1985, 34, 2645; (b) Adamson, R. N. Nature 1972, 236, 400; (c) Tai, A. W.; Lien, E. J.; Lai, M. C.; Khwaja, T. J. Med. Chem. 1984, 27, 236; (d) Chern, J. W.; Leu, Y. L.; Wang, S. S.; Lou, R.; Lee, C. F.; Tsou, P. C.; Hsu, S. C.; Liaw, Y. C.; Lin, H. W. J. Med. Chem. 1997, 40, 2276.

120. (a) Crane, B. R.; Arvai, A. S.; Gachhui, R.; Wu, C. Q.; Ghosh, D. K.; Getzoff, E. D.; Stuehr, D. J.; Tainer, J. A. Science 1997, 278, 425; (b) Crane, B. R.; Arvai, A. S.; Ghosh, D. K.; Wu, C.; Getzoff, E. D.; Stuehr, D. J.; Tainer, J. A. Science 1998, 279, 2121; (c) Fischmann, T. O.; Hruza, A.; Niu, X. D.; Fossetta, J. D.; Lunn, C. A.; Dolphin, E.; Prongay, A. J.; Reichert, P. R.; Lundell, D. J.; Narula, S. K.; Weber, P. C. Nat. Struct. Biol. 1999, 6, 223; (d) Raman, C. S.; Li, H.; Martasek, P.; Kral, V.; Masters, B. S. S.; Poulos, T. L. Cell 1998, 95, 939; (e) Li, H. Y.; Raman, C. S.; Glaser, C. B.; Blasko, E.; Young, T. A.; Parkinson, J. F.; Whitlow, M.; Poulos, T. L. J. Biol. Chem. 1999, 274, 21276.

121. (a) Clement, B.; Schultze-Mosgau, M.-H.; Wohlers, H. Biochem. Pharmacol. 1993, 46, 2249; (b) Clement, B.; Boucher, J. L.; Mansuy, D.; Harsdorf, A. Biochem. Pharmacol. 1999, 58, 439; (c) Mansuy, D.; Boucher, J. L.; Clement, B. Biochimie 1995, 77, 661.

122. Yarbro, J. W.; Kennedy, B. J.; Barnum, C. P. Proc. Natl. Acad. Sci. U.S.A. 1965, 53, 1033.

123. (a) Nishida, T.; Nagasue, N.; Arimatsu, T.; Nagano, H.; Lzumi, S.; Okura, N.; Matsumura, T.; Yakushiji, M. Nippon Sanka Fujinka Gakkai Zasshi 1989, 4, 590; (b) Currie, J. L.; Blessing, J. A.; McGehee, R.; Soper, J. T.; Berman, M. Gynecol. Oncol. 1996, 61, 94; (c) Piver, M.; Khalil, M.; Emrich, L. J. J. Surg. Oncol. 1989, 42, 120; (d) Boyd, A. S.; Neldner, K. H. J. Am. Acad. Dermatol. 1991, 25, 518.

124. Layton, A. M.; Sheehan-Dare, R. A.; Goodfield, M. J.; Cotterill, J. A. Br. J. Dermatol. 1989, 121, 647.

125. (a) DeSesso, J. M.; Goeringer, G. C. Reprod. Toxicol. 1990, 4, 145; (b) Cui, L.; Loatelli, L.; Xie, M. Y.; Sommadossi, J. P. J. Pharmacol. Exp. Ther. 1997, 280, 1228; (c) Lori, F.; Malykh, A.; Cara, A.; Sun, D.; Weinstein, J. N.; Lisziewicz, J.; Gallo, R. C. Science 1994, 266, 801.

126. Xu, Y.; Mull, C. D.; Bonifant, C. L.; Yasaki, G.; Palmer, E. C.; Shields, H.; Ballas, S. K.; Kim-Shapiro, D. B.; King, S. B. J. Org. Chem. 1998, 63, 6452.

127. (a) Reilly, E. L. German Patent 1085 166, July 14, 1960; Chem.Abstr. 1962, 56, 4594; (b) Drago, R. S.; Ragsdale, R. 0.;E yman, D. P. J.Am. Chem. Soc. 1961, 
83, 4337-4339. (c) Longhi, R.; Ragsdale, R. 0.; Drago,R. S. Inorg. Chem. 1962, 1, 768-770; (d) Ragadale,R. O.;Karstetter, B. R.; Drago, R. S. Inorg. Chem. 1966, 4, 420-422.

128. (a) Drago, R. S.; Paulik, F. E. J. Am. Chem. Soc. 1960, 82, 96-98; (b) Drago, R. S.; Karstetter, B. R. J. Am. Chem. Soc. 1961, 83, 1819-1822. (c) Joseph A. Hrabie, J. A.; Klose, J. R. J. Org. Chem. 1993, 58, 1472-1476.

129. (a) Mnragos, C. M.; Morley, D.; Wink, D. A.; Dunams, T. M.; Saavedra, J. E.; Hoffman, A.; Bove, A. A.; Isaac, L.; Hrabie, J. A.; Keefer, L. K. J.Med. Chem. 1991, 34, 3242-3247; (b) Moncada, S.; Palmer, R. M. J.; Higgs, E. A. Pharmacol. Rev. 1991, 43, 109-142. (c) Wink, D. A.; Kasprzak, K. S.; Maragos, C. M.; Elespuru, R. K.; Misra, M.; Dunams, T. M.; Cebula, T. A.; Koch, W. H.; Andrews, A. W.; Allen, J. S.; Keefer, L. K. Science, 1991, 254, 1001-1003.

130. Maragos,C. M.; Wang, J. M.; Hrabie, J. A.; Oppenheim, J. J.; Keefer L. K. Cancer Res. 1993, 53, 564-568.

131. Kumar, N.; Vandana Bhalla, V.; Manoj Kumar, M. Coord. Chem. Rev. 2013, 257, 2335-2347

132. (a) Hilderbrand, S.A.; Lim, M.H.; Lippard, S.J. J. Am. Chem. Soc. 2004, 126, 4972; (b) Lim, M. H.; Lippard, S. J. Inorg. Chem. 2004, 43, 6366; (c) Franz, K.J.; Singh, N.; Spingler, B.; Lippard, S.J. Inorg. Chem. 2000, 39, 4081.

133. (a) Fernández-Moreira, V.; Thorp-Greenwood, F. L.; Amoroso, A. J.; Cable, J.; Court, J. B.; Gray, V.; Hayes, A. J.; Jenkins, R. L.; Kariuki, B. M.; Lloyd, D.; Millet, C. O.; Williams, C. Ff.; Coogan, M. P. Org. Biomol. Chem. 2010, 8, 3888; (b) Stephenson, K. A.; Banerjee, S. R.; Besenger, T.; Sogbein, O. O.; Levadala, M. K.; McFarlane, N.; Lemon, J. A.; Boreham, D. R.; Maresca, K. P.; Brennan, J. D.; Babich, J. W.; Zubieta, J.; Valliant, J. F. J. Am. Chem. Soc. 2004, 126, 8598; (c) Ferri, E.; Donghi, D.; Panigati, M.; Prencipe, G.; D’Alfonso, L.; Zanoni, I.; Baldoli, C.; Maiorana, S.; D’Alfonso, G.; Licandro, E. Chem. Commun. 2010, 46, 6255

134. (a) Ferri, E.; Donghi, D.; Panigati, M.; Prencipe, G.; D’Alfonso, L.; Zanoni, I.; Baldoli, C.; Maiorana, S.; D’Alfonso, G.; Licandro, E. Chem. Commun. 2010, 46, 6255; (b) Thorp-Greenwood, F. L.; Coogan, M. P.; Mishra, L; Kumari, N; Rai, G; Saripella, S. N. J. Chem. 2012, 36, 64. 


\section{2. (phen) $\operatorname{Re}\left(\mathrm{PPh}_{3}\right)(\mathrm{CO})_{2} \mathrm{OSO}_{2} \mathrm{CF}_{3}$ \\ FLUORESCENT NO SENSOR.}

\subsection{ABSTRACT}

The compound (phen) $\mathrm{Re}\left(\mathrm{PPh}_{3}\right)(\mathrm{CO})_{2} \mathrm{OSO}_{2} \mathrm{CF}_{3}$ (1), which can be obtained photochemically or thermally from (phen) $\mathrm{Re}(\mathrm{CO}){ }_{3} \mathrm{OSO}_{2} \mathrm{CF}_{3}$ shows ratiometric fluorescence sensing response upon NO addition, which was added in the form of NOdonor Z-1-N-methyl-N-[6-(N-methylammoniohexyl)amino]diazen-1-ium-1,2-diolate (MAHMA-NONOate). Addition of $10-150 \mu \mathrm{M}$ of NO-donor into a $25 \mu \mathrm{M}$ solution of (phen) $\mathrm{Re}\left(\mathrm{PPh}_{3}\right)(\mathrm{CO})_{2} \mathrm{OSO}_{2} \mathrm{CF}_{3}$ in $25 \% \mathrm{v} / \mathrm{v}$ acetone/phosphate saline (PBS) buffer gave fluorescence quenching at $518 \mathrm{~nm}$, and fluorescene enhancement at $426 \mathrm{~nm}$ upon excitation at $360 \mathrm{~nm}$. The FT-IR spectra after reaction with NO showed bands at 1720 $\mathrm{cm}^{-1}$ and $1631 \mathrm{~cm}^{-1}$ that can be ascribed to Re-NO complex formation. The ${ }^{1} \mathrm{H}-\mathrm{NMR}$ and ${ }^{19}$ F-NMR spectra after reaction with NO showed characteristic resonances for noncoordinated triflate and $\mathrm{PPh}_{3}$, suggesting that the organometallic product formed upon reaction with NO releases both these ligands. Fluorescence microscopy studies on Rat Aortic Endothelial Cells in 2\% v/v acetone/phosphate saline (PBS) buffer showed distinct incorporation of the dye into cells. Compound $\mathbf{1}$ is an isolable complex, which is stable at room temperature and therefore can be used as an NO sensing organometallic dye, as prepared, without further treatment.

\subsection{INTRODUCTION}

Nitric Oxide sensing has been a topic of intense research interest since the early 90’s (references 1-16 in Chapter 1). Diaminofluoresceins (DAF) are the most commonly used dyes for fluorescent detection, because of their high sensitivity and good cell 
absorptivity. ${ }^{1}$ However DAF-like sensors have a complex NO sensing mechanism, that relies on activation of $\mathrm{NO}$ with dioxygen. ${ }^{2}$ Another strategy for selective sensing of NO is by using metal-containing dyes, such as fluorescein containing-Cu(II) and $\mathrm{Fe}(\mathrm{III})$ centers introduced by Lippard et $a l^{3}$ Surprisingly, third-row transition metal organometallics, have found very limited application to NO sensing, despite their wellstudied photophysical properties and ability to bind NO. Several transition metal nitrosyl complexes have been reported, ${ }^{4}$ including some Re(I) complexes. ${ }^{5}$ There are only a few reported examples of mixed-ligand phosphine/nitrosyl rhenium complexes. ${ }^{6}$ These complexes are typically synthesized from rhenium nitrosyls, but not vice-versa. ${ }^{7}$

Several $\operatorname{Re}(\mathrm{I})$ complexes have photoluminescent properties $^{8}$ and exhibit photoactivity in solution because of metal-to-ligand charge transfer (MLCT). ${ }^{9}$ Control of $\operatorname{Re}(\mathrm{I})$ photochemical, photophysical, and electrochemical properties has generated interest because of the use of these complexes as electro- and photocatalysts. ${ }^{10}$ Typically, Re-phosphine complexes have higher quantum yields than $[\mathrm{Ru}(\mathrm{bipy}) 3]^{2+} \cdot{ }^{11}$ Excited state properties of diimine rhenium(I) carbonyl complexes occur in the lowest triplet excited state, because of non-radiative processes that occur from the highest vibrational energy levels. ${ }^{12}$ The nature of the $\pi$-acceptor ligand (diimine or bridging ligand) determines the photophysical/photochemical properties. Mononuclear diimine rhenium(I) carbonyl complexes have quantum yields varying from 0.001 to 0.1 and long emission lifetimes (10 ns to $1 \mu \mathrm{s}$ ) as result of the lowest energy MLCT. ${ }^{13}$ There are two general methods for tuning the metal complex photophysical properties: (a) using a ligand with a different field strength to adjust the energy of the bonding orbitals in the metal center; (b) adding different substituents to the diimine ligand to alter the $\pi^{*}$ orbital energy level, producing 
a change in the absorption/emission wavelength, within the desired window range. ${ }^{13}$ Recently, a $\operatorname{Re}(\mathrm{I})$-phenanthroline-tricarbonyl-pyridine complex showed fluorescence enhancement upon NO addition. The fluorescence change is a result of triazole formation, occurring after oxidative reaction with activated $\mathrm{NO}$ (in the form of $\mathrm{N}_{2} \mathrm{O}_{3}$ ) in a similar fashion as DAF-FM. ${ }^{14}$ This compound was not found to be responsive in cells upon exogenous NO-donor addition. Our research group has recently proposed a new method for NO sensing and detection based on the favorable photophysical and NO complexation properties of metal polypyridine complexes and analogs. ${ }^{15}$

The triphenylphosphine organometallic complex (phen) $\mathrm{Re}\left(\mathrm{PPh}_{3}\right)(\mathrm{CO})_{2} \mathrm{OSO}_{2} \mathrm{CF}_{3}$ (1) introduced in this Chapter showed fluorescence responses, that can be ascribed directly to NO complexation by Re, as indicated by NMR and IR spectroscopic studies. 1 also has the additional advantages that it can be prepared in an isolable form, and is stable at room temperature. Preliminary fluorescence microcopy studies in cells have shown good absorptivity, thus indicating its promise a fluorescent probe for nitric oxide imaging.

\subsection{EXPERIMENTAL SECTION}

\subsubsection{Materials and Methods}

(phen) $\mathrm{Re}(\mathrm{CO})_{3} \mathrm{Cl}$ and (phen) $\mathrm{Re}(\mathrm{CO})_{3} \mathrm{OSO}_{2} \mathrm{CF}_{3}$ were prepared as previously

reported and were found spectroscopically identical to the reported compounds. ${ }^{16,17}$ All other materials (purchased from Aldrich Chemical Co., ACROS Organics, Cayman Chemicals, or Alfa Aesar) were standard reagent grade and were used without further purification, unless otherwise noted. Tetrahydrofuran (THF) used for the preparation of 1, was anhydrous (purchased as an Aldrich Sure-Seal ${ }^{\mathrm{TM}}$ product), or distilled and dried 
before use. PBS buffers $\mathrm{A}(0.002 \mathrm{M}$ in total phosphate , $\mathrm{pH}=7.2,[\mathrm{NaCl}]=0.04 \mathrm{M})$ and $\mathrm{B}(0.002 \mathrm{M}$ in total phosphate, $\mathrm{pH}=7.2,[\mathrm{NaCl}]=0.02 \mathrm{M})$ were prepared from $\mathrm{K}_{2} \mathrm{HPO}_{4}$, $\mathrm{KH}_{2} \mathrm{PO}_{4}$, and $\mathrm{NaCl}$ (all from Fisher Scientific). Water used for preparation of the buffers and experiments with Nitric oxide was filtered water $\left(18 \mathrm{M} \Omega \cdot \mathrm{cm}^{-1}\right)$ produced from a Millipore ${ }^{\circledR}$ nanopure diamond lab water system (Barnstead Thermolyne Corporation, Dubuque, IA). Deuterated solvents were purchased from Cambridge Isotope Laboratories Ltd. Rat Aortic Endothelial Cells were acquired from Cell Biologics. Fetal Bovine Serum (FBS) and DMSO were bought from ATCC Inc. Penicillin-Streptomycin and endothelial cell growth supplement from bovine neural tissue were purchased from Sigma-Aldrich. DMEM/F-12 medium, PBS (for fluoresence microscopy experiments) and Trypsin (0.05\% EDTA) were purchased from GIBCO/Invitrogen Inc. Cell culture flasks and dishes were purchased from Fisher Scientific. Photochemical reactions were carried out using a Rayonet photochemical reactor (Southern New England Ultraviolet, model RPR100), equipped with a cooling fan and 10 phosphor-coated low-pressure mercury lamps $\left(\lambda_{\max }=300 \mathrm{~nm}\right.$, light intensity $\left.=5.2 \pm 0.1 \times 10^{6} \mathrm{photon} / \mathrm{sec} / \mathrm{cm}^{3}\right)$. Fluorescence Spectra were recorded on a Jobin Yvon Horiba Fluoromax-3 fluorimeter. Fluorescence Microscopy was performed using an Olympus IX-81 Fluorescence Microscope and analyzed with IPLab v 4.04 Scientific Imaging Processing Software. Frequency-domain fluorescence lifetime measurements were performed using a ChronosFD spectrofluorometer (ISS, Champaign, IL). Samples were excited with a $370 \mathrm{~nm}$ modulated diode, and emission was collected using $400 \mathrm{~nm}$ long-pass filters (Andover, Salem, NH). The ${ }^{1} \mathrm{H}$ and the ${ }^{13} \mathrm{C}-\mathrm{NMR}$ spectra were recorded on a $400 \mathrm{MHz}$ Bruker NMR spectrometer and were referenced, using the residual solvent resonances. All 
chemical shifts, $\delta$, are reported in ppm. The FT-IR spectra were recorded on a PerkinElmer Spectrum 100 FT-IR Spectrometer.

\subsubsection{Synthesis of (phen) $\mathrm{Re}\left(\mathrm{PPh}_{3}\right)(\mathrm{CO})_{2} \mathrm{OSO}_{2} \mathrm{CF}_{3}$ :}

Method 1 - Thermal Substitution: $20.0 \mathrm{mg}$ of (phen) $\mathrm{Re}(\mathrm{CO})_{3} \mathrm{OSO}_{2} \mathrm{CF}_{3}(33.3 \mu \mathrm{mol})$ and $10 \mathrm{~mL}$ of THF (final concentration: $3.33 \mathrm{mM}$ ) were placed in a $50 \mathrm{~mL}$ round bottom flask, under $\mathrm{N}_{2}$ flow. The solution was refluxed for $48 \mathrm{~h}$. The color changed during reflux from yellow to amber. The volatiles were evaporated to dryness and $1.0 \mathrm{~mL}$ of dichloromethane was added. The product was precipitated at room temperature by adding $4.0 \mathrm{~mL}$ of diethyl ether. The bright yellow powder product was filtered and was dried in vacuo at $40{ }^{\circ} \mathrm{C} .20 .5 \mathrm{mg},(74 \%) .{ }^{1} \mathrm{H}-\mathrm{NMR}\left(\mathrm{CDCl}_{3}\right): 6.96\left(\mathrm{t}, 3 \mathrm{H},{ }^{3} \mathrm{~J}_{\mathrm{H}-\mathrm{H}}=7.0 \mathrm{~Hz}\right), 7.39$ (dd, 6H, ${ }^{3} \mathrm{~J}_{\mathrm{H}-\mathrm{H}}=7.6 \mathrm{~Hz},{ }^{3} \mathrm{~J}_{\mathrm{H}-\mathrm{H}}=2.0 \mathrm{~Hz}$ ), $7.62\left(\mathrm{dd}, 6 \mathrm{H},{ }^{3} \mathrm{~J}_{\mathrm{H}-\mathrm{H}}=12.5 \mathrm{~Hz},{ }^{3} \mathrm{~J}_{\mathrm{H}-\mathrm{H}}=7.2 \mathrm{~Hz}\right.$ ), $7.95\left(\mathrm{~d}, 2 \mathrm{H},{ }^{3} \mathrm{~J}_{\mathrm{H}-\mathrm{H}}=8.2 \mathrm{~Hz},{ }^{3} \mathrm{~J}_{\mathrm{H}-\mathrm{H}}=5.1 \mathrm{~Hz}\right), 8.36(\mathrm{~s}, 2 \mathrm{H}), 8.99\left(\mathrm{~d}, 2 \mathrm{H},{ }^{3} \mathrm{~J}_{\mathrm{H}-\mathrm{H}}=8.28 \mathrm{~Hz}\right)$, $9.14\left(\mathrm{~d}, 2 \mathrm{H},{ }^{3} \mathrm{~J}_{\mathrm{H}-\mathrm{H}}=4.7 \mathrm{~Hz}\right) \cdot{ }^{13} \mathrm{C}-\left\{{ }^{1} \mathrm{H}\right\}-\mathrm{NMR}\left(\mathrm{CDCl}_{3}\right): 128.92(p-\mathrm{CH}, \mathrm{s}, 3 \mathrm{C}), 129.15(\mathrm{~m}-$ $\left.\mathrm{CH}, \mathrm{d}, 6 \mathrm{C},{ }^{3} \mathrm{~J}_{\mathrm{C}-\mathrm{P}}=37.8 \mathrm{~Hz}\right), 131.23(\mathrm{CH}, \mathrm{s}, 2 \mathrm{C}), 132.38\left(\mathrm{o}-\mathrm{CH}, \mathrm{d}, 6 \mathrm{C},{ }^{1} \mathrm{~J}_{\mathrm{C}-\mathrm{P}}=40.5 \mathrm{~Hz}\right)$, 137.14 (CH, s, 2C), 140.04 (CH, s, 2C), $153.32(\mathrm{CH}, \mathrm{s}, 2 \mathrm{C}) .{ }^{19} \mathrm{~F}-\left\{{ }^{1} \mathrm{H}\right\}-\mathrm{NMR}\left(\mathrm{CDCl}_{3}\right)$ : 78.3 ppm FT-IR ( $\left.\mathrm{cm}^{-1}\right)$ : 2029 ( $\left.v_{\mathrm{co}}, \mathrm{s}\right), 1926$ ( $\left.v_{\mathrm{co}}, \mathrm{s}\right), 1906$ ( $\left.v_{\mathrm{co}}, \mathrm{s}\right)$, 1433, 1266, 1152, 1031, 854, 725, 638. Anal. Calc. $\mathrm{C}_{33} \mathrm{H}_{23} \mathrm{~F}_{3} \mathrm{~N}_{2} \mathrm{O}_{6}$ PReS: C 46.64\%, H 2.72\%, N 3.29\%. Found: $\quad$ C $46.28 \%, \mathrm{H} 2.32 \%, \mathrm{~N} 3.20 \%$. UV-Vis (in ethanol): $\lambda_{\max }=360 \mathrm{~nm}(\varepsilon=$ $4500 \mathrm{~mol}^{-1} \mathrm{~cm}^{-1}$ ), fluorescence emission in acetone $\left(\lambda_{\text {exc }}=360 \mathrm{~nm}\right.$ ): $585 \mathrm{~nm} \Phi_{1}=0.027$ (at $370 \mathrm{~nm}$, in 1:3 acetone/PBS buffer, using quinine sulfate in sulfuric acid $0.1 \mathrm{M}$ as reference $\left.\Phi_{\mathrm{R}}=0.546\right)$.

Lifetime method 1: Frequency dependent, useful for fast lifetimes: $\tau_{1}=21.3 \pm 0.3 \mathrm{~ns}$, $\mathrm{f}_{1}=0.0626 \pm 0.0008, \alpha_{1}=0.00294 ; \tau_{2}=389 \pm 5 \mathrm{~ns}, \mathrm{f}_{2}=0.601 \pm 0.007, \alpha_{2}=0.00155$ 
Lifetime method 2: Time-dependent, useful for slow lifetimes: $\tau_{1}=35.4 \pm 0.05 \mathrm{~ns}, \mathrm{f}_{1}=$ $0.869 \pm 0.047 ; \tau_{2}=225 \pm 0.06 \mathrm{~ns}, \mathrm{f}_{2}=0.094 \pm 0.0003 ; \tau_{3}=1995 \pm 15 \mathrm{~ns}, \mathrm{f}_{3}=0.006 \pm$ 0.00003; with fluorescence still observable after $1 \mu \mathrm{s}$. Differences between the two methods can be attributable to in the second method the values are result of an algorithmic fitting instead of real measurement.

Method 2 - Photochemical Substitution: $1.0 \mathrm{mg}$ of (phen)Re(CO) ${ }_{3} \mathrm{OSO}_{2} \mathrm{CF}_{3}(1.67 \mu \mathrm{mol})$, $3.0 \mathrm{mg}$ of triphenylphosphine and $1.00 \mathrm{~mL}$ of THF (final concentration $1.67 \mathrm{mM}$ ) were placed in a NMR tube. The solution was irradiated with a photochemical reactor $\left(\lambda_{\max }=\right.$ $300 \mathrm{~nm}$, light intensity $=5.2 \pm 0.1 \times 106$ photon $/ \mathrm{sec} / \mathrm{cm}^{3}$ ). After irradiation the color of the solution changed from yellow to amber. This solution was used without further treatment. FT-IR $\left(\mathrm{cm}^{-1}\right)$ : 2029 (vco, vs), 1921 (vco, vs), 1905 (vco, vs), 1722 (vco-o, vs), 1633 (vco-o, vs), 1437, 1279, 1261, 1180, 1118, 1030, 749, 693, 637.

\subsubsection{Fluorescence experiments with NO-donor}

In a typical experiment, $1.000 \mathrm{~mL}$ of a solution of (phen) $\mathrm{Re}(\mathrm{THF})(\mathrm{CO})_{2} \mathrm{OSO}_{2} \mathrm{CF}_{3}$ or (phen) $\mathrm{Re}\left(\mathrm{PPh}_{3}\right)(\mathrm{CO})_{2} \mathrm{OSO}_{2} \mathrm{CF}_{3}$ complex $(100 \mu \mathrm{M})$ was prepared by dilution with acetone of a $1.67 \mathrm{mM}$ THF stock solution. Aqueous PBS buffer $\mathrm{A}(\mathrm{pH}=7.2$, $[\mathrm{NaCl}]=0.04 \mathrm{M}, 2.75 \mathrm{~mL}$ to $3.00 \mathrm{~mL})$ was added. Then $0-250 \mu \mathrm{L}$ of $4 \mathrm{mM}$ stock solution of MAHMA-NONOate NO-donor, in $0.04 \mathrm{M} \mathrm{NaOH}$ were added, in order to obtain $75 \% \mathrm{v} / \mathrm{v}$ aqueous/organic solution and a final NO-donor concentration in the $0-200 \mu \mathrm{M}$ range, while the concentration of the Re complex remains constant at 25 $\mu \mathrm{M}$. No more than 10 min. elapsed from preparation of the organic phase to the addition of NO-donor. The solution was mixed thoroughly in a vortex and sonicated for $90 \mathrm{~min}$. at 
room temperature. The fluorescence of all solutions was measured at $\lambda_{\mathrm{exc}}=360 \mathrm{~nm}$ and recorded from $400 \mathrm{~nm}$ to $700 \mathrm{~nm}$. For a control experiment a $\mathrm{NaOH}$ stock solution was used instead of an NO-donor/NaOH stock, in order to account for any possible variations in the fluorescence due to minor $\mathrm{pH}$ changes. The result of the control titrations gave identical spectra for all titration points in the $1-150 \mu \mathrm{L}$ range. These experiments were also performed in identical conditions for control compounds (phen) $\mathrm{Re}(\mathrm{CO}){ }_{3} \mathrm{Cl}$ and (phen) $\mathrm{Re}(\mathrm{CO})_{3} \mathrm{OTf}$ and no significant changes were observed for their fluorescence emissions upon NO addition.

\subsubsection{Fluorescence titrations with gradual addition of NO-donor under} anaerobic and aerobic conditions.

In a typical experiment, $0.750 \mathrm{~mL}$ of a (phen) $\mathrm{Re}(\mathrm{THF})(\mathrm{CO})_{2} \mathrm{OSO}_{2} \mathrm{CF}_{3}$ or (phen) $\mathrm{Re}\left(\mathrm{PPh}_{3}\right)(\mathrm{CO})_{2} \mathrm{OSO}_{2} \mathrm{CF}_{3}$ solution $(100 \mu \mathrm{M})$ was prepared from dilution of $20 \mu \mathrm{L}$ of a $1.67 \mathrm{mM}$ THF stock solution with acetone. A quantity of $2.25 \mathrm{~mL}$ of aqueous PBS buffer $\mathrm{A}(\mathrm{pH}=7.2,[\mathrm{NaCl}]=0.04 \mathrm{M})$ was added. The fluorescence emission was monitored using $\lambda_{\text {exc }}=360 \mathrm{~nm}$. Then $3 \mathrm{mM}$ stock solution of MAHMA-NONOate NO-donor in $0.04 \mathrm{M} \mathrm{NaOH}$ was added progressively $300 \mu \mathrm{L}$ in $25 \mu \mathrm{L}$ portions. After each addition, the solution was left react for 10 minutes (approximately 3 MAHMA NONOate half-life), at room temperature before measuring fluorescence using the same parameters as above.

\subsubsection{Fluorescence titrations with low concentrations of NO-donor.}

In a typical experiment, $0.750 \mathrm{~mL}$ of a (phen)Re(THF)(CO) ${ }_{2} \mathrm{OSO}_{2} \mathrm{CF}_{3}$ or (phen) $\mathrm{Re}\left(\mathrm{PPh}_{3}\right)(\mathrm{CO})_{2} \mathrm{OSO}_{2} \mathrm{CF}_{3}$ solution $(100 \mu \mathrm{M})$ was prepared from dilution of $20 \mu \mathrm{L}$ of a $1.67 \mathrm{mM}$ THF stock solution with acetone. A quantity of $2.25 \mathrm{~mL}$ of aqueous PBS 
buffer $\mathrm{B}(\mathrm{pH}=7.2,[\mathrm{NaCl}]=0.02 \mathrm{M})$ was added. The solution was bubbled with nitrogen for 30 minutes. The fluorescence emission was monitored using $\lambda_{\text {exc }}=360 \mathrm{~nm}$. Then 3 mM stock solution of MAHMA-NONOate NO-donor in $0.04 \mathrm{M} \mathrm{NaOH}$ was added progressively $150 \mu \mathrm{L}$ in $1-25 \mu \mathrm{L}$ portions. After each addition, the solution was left react for 10 minutes (approximately 3 MAHMA NONOate half-life), at room temperature before measuring fluorescence using the same parameters as above.

\subsection{6. ${ }^{1} \mathrm{H}-\mathrm{NMR}$ and ${ }^{19} \mathrm{~F}$-NMR study of reactivity of Re complexes with NO:}

(phen) $\mathrm{Re}\left(\mathrm{PPh}_{3}\right)(\mathrm{CO})_{2} \mathrm{OSO}_{2} \mathrm{CF}_{3}(1 \mathrm{mg})$ was added to $0.05 \mathrm{~mL}$ of THF in four separate $10 \mathrm{~mL}$ vials. Acetone $(1.6 \mathrm{~mL})$ was added to each vial. Then $2.4 \mathrm{~mL}$ of PBS buffer $\mathrm{A}(\mathrm{pH}=7.2$, $[\mathrm{NaCl}]=0.04 \mathrm{M})$ and $0.2 \mathrm{~mL}$ of stock NO-donor solution $(5 \mathrm{mM}$ in $0.04 \mathrm{M} \mathrm{NaOH}$ ) were added sequentially. The solutions were vortexed for $30 \mathrm{sec}$. and then sonicated for $3 \mathrm{~h}$ at room temperature. Dichloromethane was added ( $3 \mathrm{x} 10 \mathrm{~mL})$, and the organic layer was dried with anhydrous sodium sulfate. The solvent was evaporated and the non-volatiles were taken up in chloroform-D and the NMR spectra were collected. In order to remove uncomplexed phenanthroline products and collect only the NMR spectra of complexes, the organic phases were further diluted with dichloromethane and washed with $1 \mathrm{M}$ hydrochloric acid, followed by $1 \mathrm{M}$ sodium bicarbonate, rinsed with deionized water, and dried with anhydrous sodium sulfate. The volatiles were evaporated, the solid residues were redissolved in chloroform-D, and the ${ }^{1} \mathrm{H}$-NMR and ${ }^{19} \mathrm{~F}-\mathrm{NMR}$ spectra were recorded. These experiments were also performed under identical conditions for control compounds (phen) $\mathrm{Re}(\mathrm{CO}){ }_{3} \mathrm{Cl}$ and (phen)Re(CO) ${ }_{3} \mathrm{OTf}$. After $\mathrm{HCl} / \mathrm{NaHCO}_{3}$ washing no changes were observed in the ${ }^{1} \mathrm{H}$ - 
NMR spectrum of (phen) $\operatorname{Re}(\mathrm{CO}){ }_{3} \mathrm{Cl}$, while for (phen) $\operatorname{Re}(\mathrm{CO})_{3} \mathrm{OTf}$, resonances identical to (phen) $\mathrm{Re}(\mathrm{CO}){ }_{3} \mathrm{Cl}$ were observed.

\subsubsection{Fluorescence microscopy studies of (phen) $\mathrm{Re}\left(\mathrm{PPh}_{3}\right)(\mathrm{CO})_{2} \mathrm{OSO}_{2} \mathrm{CF}_{3}$}

\section{in Rat Aortic Endothelial Cells:}

Cell preparation: Rat Aortic Endothelial Cells were grown in Dublecco's Modified Eagle Medium: Nutrient Mixture F-12 medium (DMEM/F-12) supplemented with 10\% Fetal Bovine Serum (FBS), 1\% penicillin-streptomycin, and endothelial cell growth supplement in a humidified tissue culture incubator at $37^{\circ} \mathrm{C}$ equilibrated with $95 / 5 \mathrm{v} / \mathrm{v} \%$ Air/ $\mathrm{CO}_{2}$ mixture. Cells were cultured in $35 \times 10 \mathrm{~mm}$ dishes seeded with $2 \times 10^{5}$ cells and used at $60-80 \%$ confluency.

Solution Preparation: A $500 \mu \mathrm{M}$ solution of $\mathrm{Re}(\mathrm{phen})(\mathrm{THF})(\mathrm{CO})_{2} \mathrm{OSO}_{3} \mathrm{CF}_{3}$ in THF/acetone (Solution B) was prepared from dilution of $62.5 \mu \mathrm{L}$ of a stock solution of Re(phen)(THF)(CO) ${ }_{2} \mathrm{OSO}_{3} \mathrm{CF}_{3}$ in THF $\left(1.6 \times 10^{-3} \mathrm{M}\right.$ - Solution A) with $138 \mu \mathrm{L}$ of acetone. This solution was added to $800 \mu \mathrm{L}$ of commercial $10 \mathrm{mM}$ PBS buffer solution (pH=7.2), resulting in a final $100 \mu \mathrm{M}$ solution of $\mathbf{1}$ in $80 / 14 / 6 \mathrm{v} / \mathrm{v}$ aqueous buffer/acetone/THF. This new solution was further diluted 1/10 v/v with PBS Buffer before adding it to the cells, resulting in a final solution of $10 \mu \mathrm{M}$ of 1 in 98/1.4/0.6 v/v aqueous PBS buffer/acetone/THF (Solution C). Various quantities of $6 \mathrm{mM}$ NO-donor solution (MAHMA-NONOate) in $0.01 \mathrm{M} \mathrm{NaOH}$ were diluted with $0.01 \mathrm{M} \mathrm{NaOH}$ to give 0, 100, $500 \& 1000 \mu \mathrm{M}$ solutions of NO-donor (Solutions D-G).

Loading of solution C (10 $\mu \mathrm{M}$ of Re complex 1): The old media were poured out from the plates; then the plates were rinsed twice with $1 \mathrm{~mL}$ of $10 \mathrm{mM}$ PBS $(\mathrm{pH}=7.2)$. Then 100 
$\mu \mathrm{L}$ of DMEM/FBS/Penicillin Media, $800 \mu \mathrm{L}$ of PBS $10 \mathrm{mM}$, and $100 \mu \mathrm{L}$ of Re complex stock (Solution C) were added with this specific order. The plates were incubated for 75 min. at $37{ }^{\circ} \mathrm{C}$ equilibrated with $95 / 5$ v/v\% Air/CO2 mixture.

Fluorescence Microscopy Measurements: Each plate was placed in the fluorescence microscope and measured under a filter an excitation wavelength of $350 \mathrm{~nm}$ (330-380 nm window) and an emission window of 440-460 nm. The images were collected with a total exposure time of $2668 \mathrm{~ms}$ and 20X zoom.

\subsection{RESULTS AND DISCUSSION}

\subsubsection{Synthesis of (phen) $\operatorname{Re}\left(\mathrm{PPh}_{3}\right)(\mathrm{CO})_{2} \mathrm{OSO}_{2} \mathrm{CF}_{3}(1)$}

Complex 1 was synthesized by photochemical or thermal substitution of CO from (phen) $\mathrm{Re}(\mathrm{CO})_{3} \mathrm{SO}_{3} \mathrm{CF}_{3}$. Only the thermal preparation was performed on a larger scale and led to an isolable quantity of the product. However the product was found to have identical UV-Vis and NMR spectra, regardless of the preparation method. The isolated product was characterized by ${ }^{1} \mathrm{H}-\mathrm{NMR},{ }^{13} \mathrm{C}-\mathrm{NMR}$, and $\mathrm{FT}-\mathrm{IR}$, and its purity was confirmed by elemental analysis.

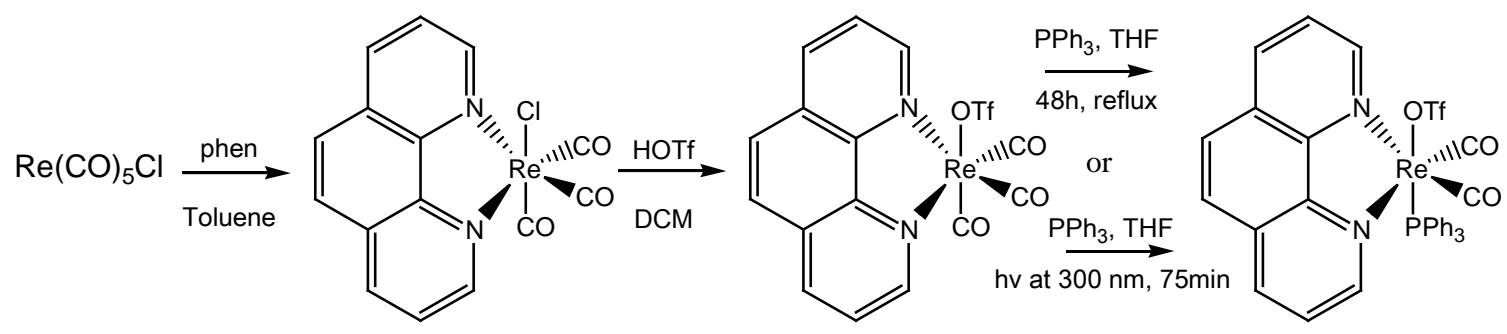

Scheme 2.1 Synthesis of (phen) $\mathrm{Re}\left(\mathrm{PPh}_{3}\right)(\mathrm{CO})_{2} \mathrm{OSO}_{2} \mathrm{CF}_{3}$ (1)

\subsubsection{Fluorescence studies with addition of NO-donor}

Complex 1 showed significant fluorescence response to NO addition. The fluorescence spectrum of 1 in acetone/buffer 25/75 v/v shows one emission at $514 \mathrm{~nm}$ 
( $\Phi=0.03$ ). Figure 2.1 shows the effects on the fluorescence spectrum of $\mathbf{1}$ upon NO gradual addition, in the form of NO-donor MAHMA-NONOate (0 - 150 $\mu \mathrm{M})$ dissolved in $0.04 \mathrm{M} \mathrm{NaOH}$. The emission at $514 \mathrm{~nm}$ decreases rapidly upon NO addition and its intensity shows linear dependence with NO-donor concentration, thus allowing NO quantification in the $0-150 \mu \mathrm{M}$ range (Figure 2.2).

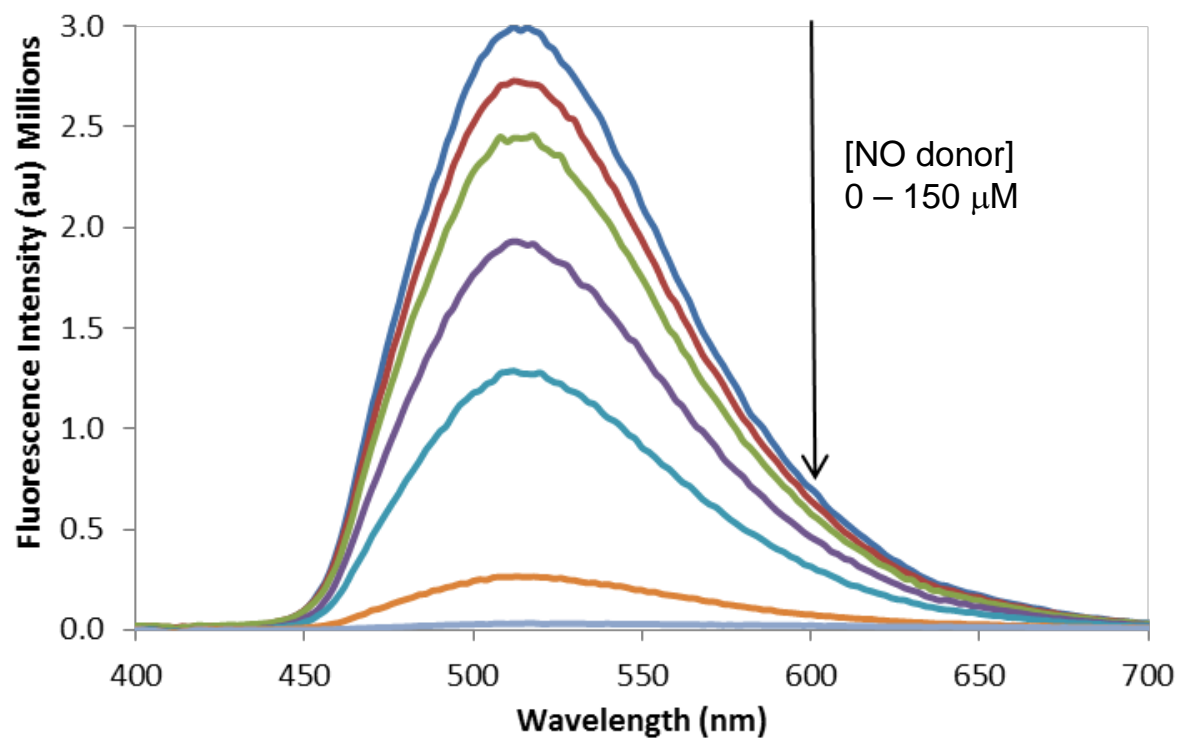

Figure 2.1 Fluorescence Response upon NO-donor addition $(0-150 \mu \mathrm{M})$ to $20 \mu \mathrm{M}$ of $\mathrm{Re}$ complex 1 in acetone/PBS aqueous buffer $25 / 75 \mathrm{v} / \mathrm{v},\left(\lambda_{\text {exc }}=360 \mathrm{~nm}\right)$

A control experiment in the absence of NO-donor, (addition of $0.04 \mathrm{M} \mathrm{NaOH}$ ) shows no observable changes in fluorescence intensity, proving that the sensor responds to NO-donor addition and not any other substance present in the matrix of the added solution (Figure 2.2). It should also be noted that by using a $0.002 \mathrm{M}$ PBS buffer, in $0.04 \mathrm{M} \mathrm{NaCl}$, and a $\mathrm{NaOH} 0.04 \mathrm{M}$ solution for the $\mathrm{N}$ donor no precipitation is obdserved under the concentration range of these experiments. These experiments were also performed in identical conditions for control compounds (phen) $\mathrm{Re}(\mathrm{CO}){ }_{3} \mathrm{Cl}$ and (phen) $\mathrm{Re}(\mathrm{CO})_{3} \mathrm{OTf}$ and no significant changes were observed at their fluorescence 
emissions at $565 \mathrm{~nm}$, upon NO addition, indicating that the presence of a labile L ligand is necessary for reaction with NO.

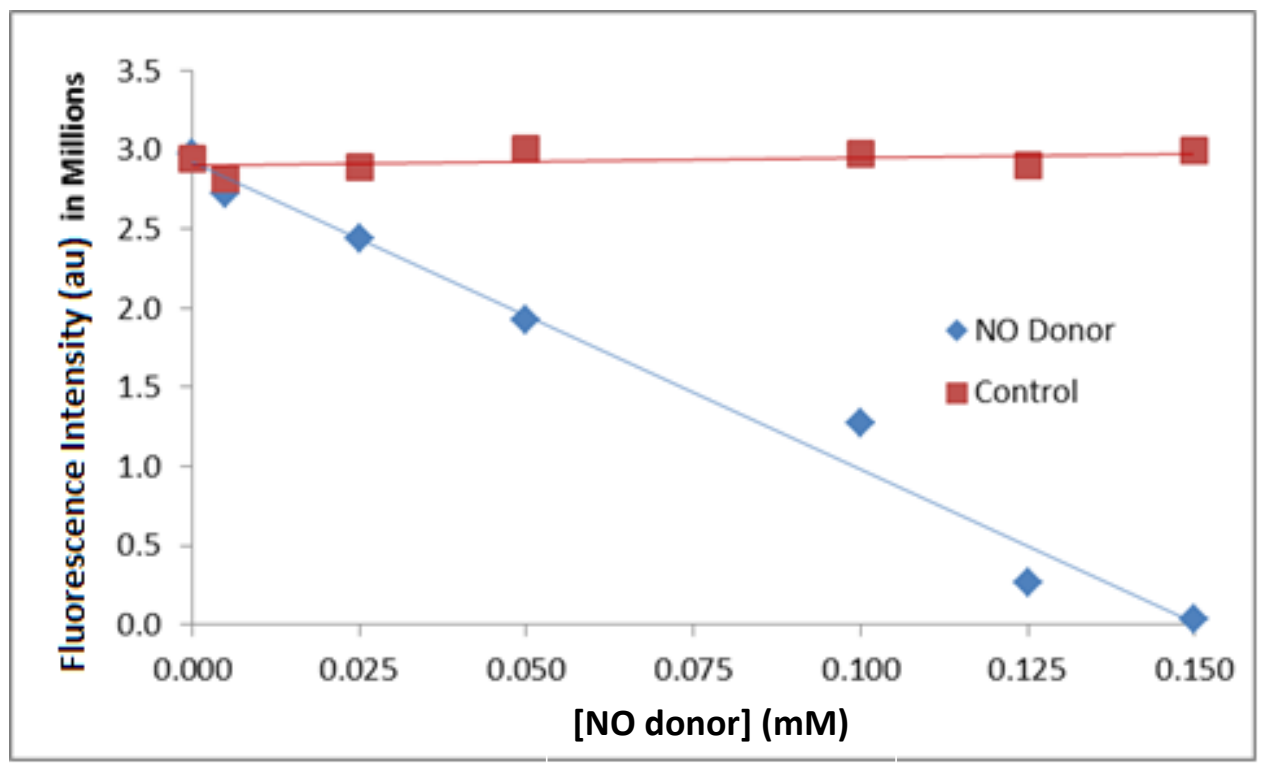

Figure 2.2 Observed Fluorescence intensities of $1(20 \mu \mathrm{M})$ at $\lambda$ max $=514 \mathrm{~nm}$ upon NO-donor addition vs addition of control solution $(\mathrm{NaOH} 0.04 \mathrm{M}) . \lambda$ exc $=360 \mathrm{~nm}$.

Complex 1 prepared photochemically on a $1 \mathrm{mg}$ scale (1.67 mM in THF) showed also significant fluorescence response to $\mathrm{NO}$ addition both at $560 \mathrm{~nm}$ (decrease) and at $455 \mathrm{~nm}$ (increase). The fluorescence spectrum of 1 in THF/acetone/buffer 2/23/75 shows one emission at $562 \mathrm{~nm}$. The shift in emission compared to the isolated complex could be ascrtibed both to the difference in the solvent system, and the presence of unreacted (phen) $\mathrm{Re}(\mathrm{CO})_{3} \mathrm{SO}_{3} \mathrm{CF}_{3}$, or (phen) $\mathrm{Re}(\mathrm{CO})_{2}(\mathrm{THF}) \mathrm{SO}_{3} \mathrm{CF}_{3}$ which emit at 562 $\mathrm{nm}$ and $535 \mathrm{~nm}$ respectively (see Chapter 3). Figure 2.3 shows the effects on the fluorescence spectrum of $\mathbf{1}$ upon NO gradual addition, in the form of NO-donor MAHMA-NONOate $(0-150 \mu \mathrm{M})$ dissolved in $0.04 \mathrm{M} \mathrm{NaOH}$. The emission at $560 \mathrm{~nm}$ decreases rapidly upon NO addition (with a gradual low-energy shift), while the increase 
at $452 \mathrm{~nm}$ is more pronounced a higher NO-donor concentrations leading to a pseudoratiometric response, and an "isosbestic area" at $485 \mathrm{~nm}$.

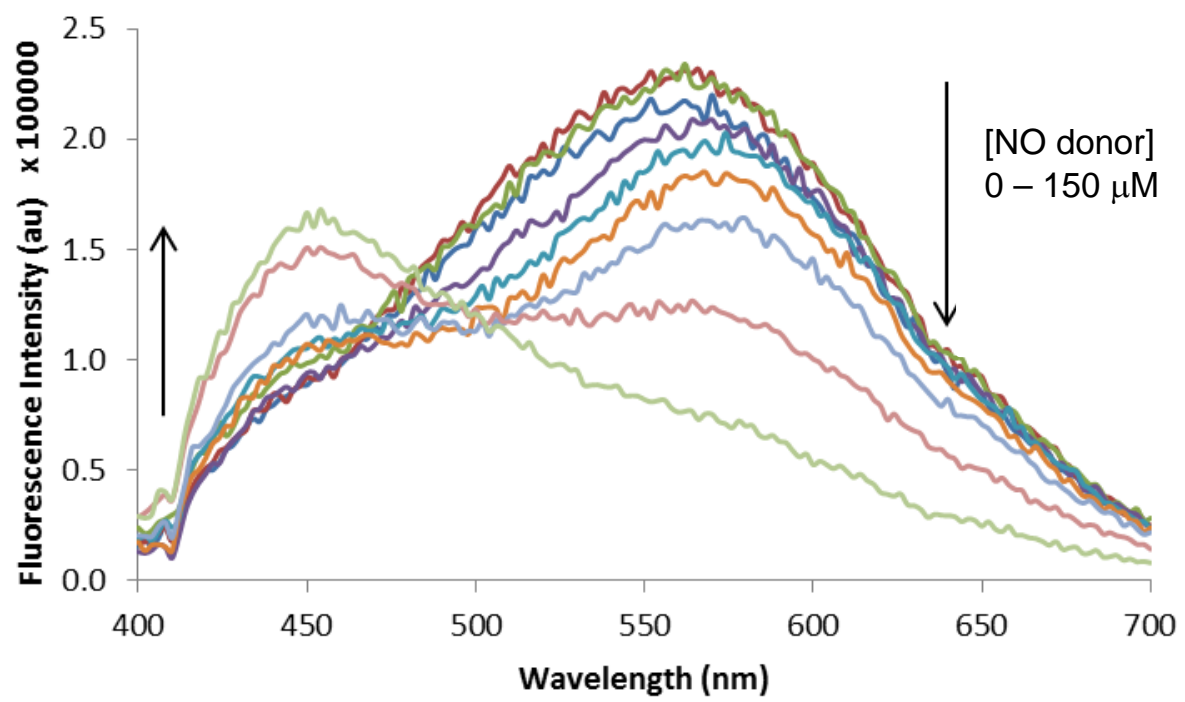

Figure 2.3 Fluorescence Response upon NO-donor addition $(0-150 \mu \mathrm{M})$ to $25 \mu \mathrm{M}$ of Re complex 1 prepared photochemically, used as THF solution, in acetone/PBS aqueous buffer $25 / 75 \mathrm{v} / \mathrm{v}\left(\lambda_{\text {exc }}=360 \mathrm{~nm}\right)$

The plot of intensity ratio for the two signals at $452 \mathrm{~nm}$ and $562 \mathrm{~nm}$ vs. NO-donor concentration, (Figure 2.4) allows NO quantification using the ratio of the two responses. The rapid increase on the ratiometric response for higher NO concentrations are result of to the different range of NO in which the intensities at 565 $\mathrm{nm}$ and $454 \mathrm{~nm}$ are most sensitive. Specifically the $562 \mathrm{~nm}$ intensity decrease shows high sensitivity for low NO concentration, while the increase at $452 \mathrm{~nm}$ becomes more notable for high concentrations. 


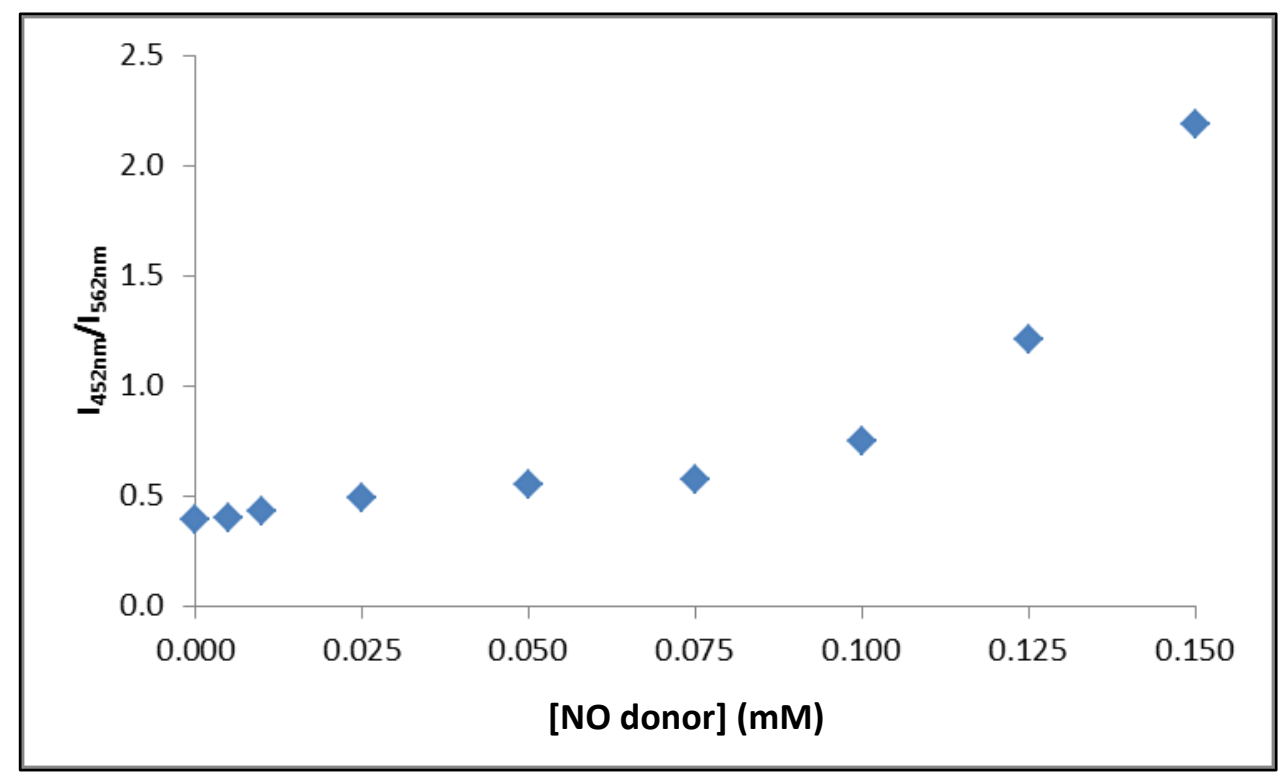

Figure 2.4 Fluorescence intensity ratio of $454 \mathrm{~nm} / 562 \mathrm{~nm}$ for $\mathbf{1}(25 \mu \mathrm{M})$ with NO-donor addition $(0-150 \mu \mathrm{M})$

\subsubsection{Fluorescence titration with gradual addition of NO-donor under anaerobic and aerobic conditions.}

Complex 1 showed stability under aqueous media, $37^{\circ} \mathrm{C}$ and $\mathrm{pH} 7$. Figures 2.5 and 2.6 show the effects on the fluorescence spectrum of $\mathbf{1}$ upon titrations with NO, in the form of NO-donor MAHMA-NONOate (0-150 $\mu \mathrm{M})$ dissolved in $0.04 \mathrm{M} \mathrm{NaOH}$, added in $25 \mu \mathrm{L}$ portions after 10 minutes intervals, under anaerobic (Figure 2.5) or aerobic (Figure 2.6) conditions. The emission at $514 \mathrm{~nm}$ decreases rapidly upon NO addition and its intensity shows linear dependence with NO-donor concentration. The experiment was performed under nitrogen atmosphere in order to avoid $\mathrm{NO}$ oxidation to $\mathrm{N}_{2} \mathrm{O}_{3}$. 


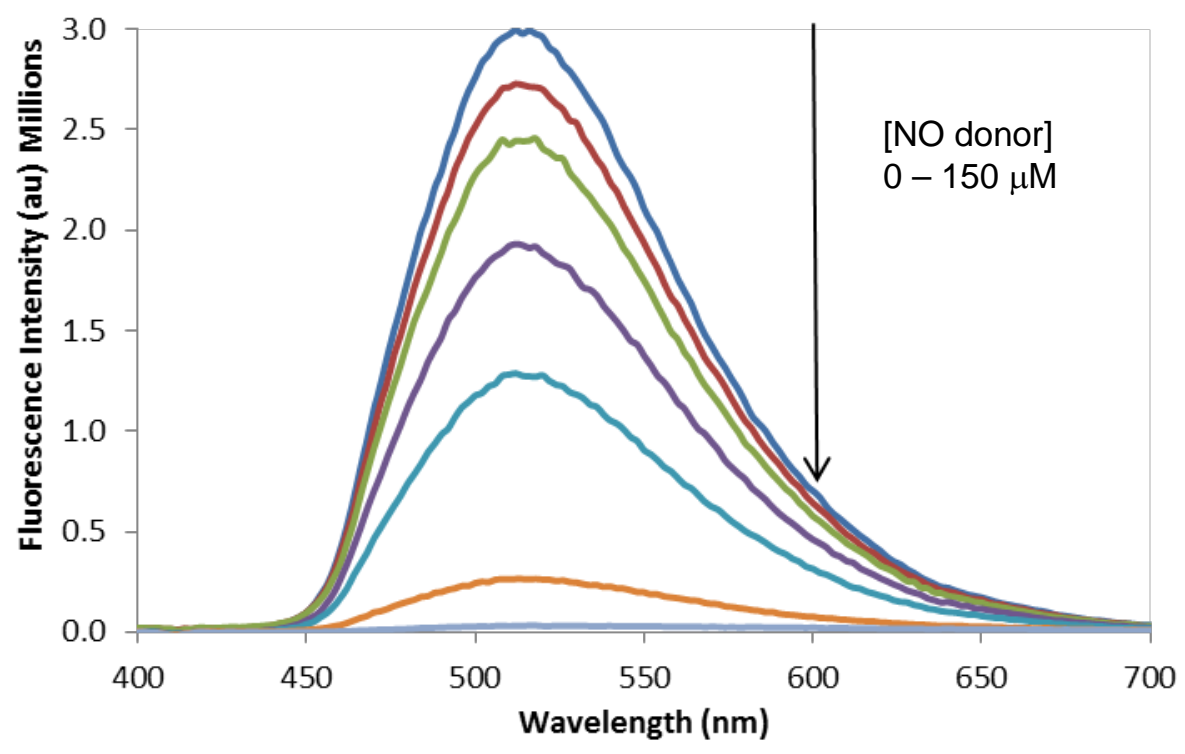

Figure 2.5 Fluorescence Spectra of $10 \mu \mathrm{M} 1$ with NO, acetone/buffer 25:75, $\lambda_{\text {exc }}=360 \mathrm{~nm}$, under nitrogen

Quenching of the $514 \mathrm{~nm}$ emission, was observed under both anaerobic and aerobic conditions. However, under anaerobic conditions (Fig. 2.5) the effect is more pronounced, indicating that oxygen is not needed in order for the sensing of NO to take place. Such observation is consistent with a mechanism that does not involve $\mathrm{O}_{2}$ activated intermediates such as $\mathrm{NO}_{2}$ and $\mathrm{N}_{2} \mathrm{O}_{3}$, and perhaps implies that sensing is a direct result of a reaction of NO with the Re(I) dye. 


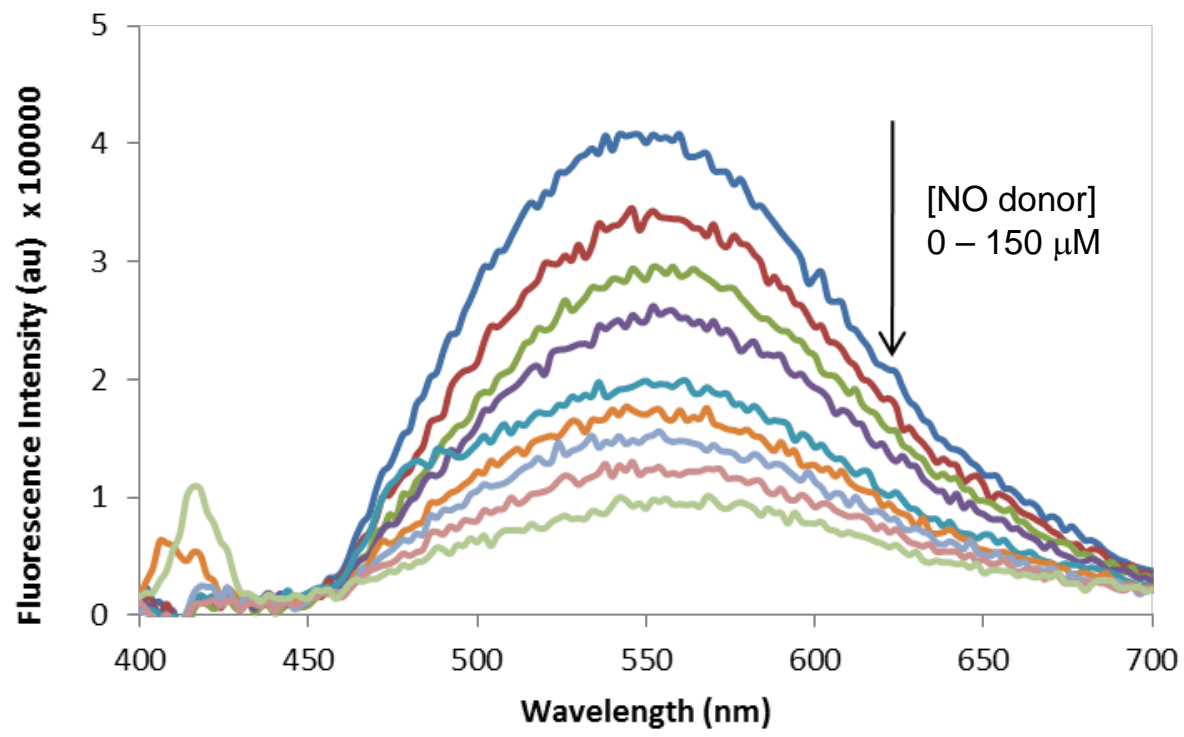

Figure 2.6 Fluorescence Spectra of $10 \mu \mathrm{M} 1$ with NO-donor, acetone/buffer 25:75, $\lambda_{\text {exc }}$ $=360 \mathrm{~nm}$

In the comparison plot (Figure 2.7) of I514 from titration spectra under nitrogen (Figure 2.5) and under air (Figure 2.6) the consistency of response in aerobic and anaerobic conditions is readily observable. The higher sensitivity for low NO concentrations under anaerobic conditions could be attributed to the rapid reaction of NO with $\mathrm{O}_{2}$ forming non-reactive oxidized products, such as $\mathrm{NO}_{2}$ and $\mathrm{N}_{2} \mathrm{O}_{3}$.

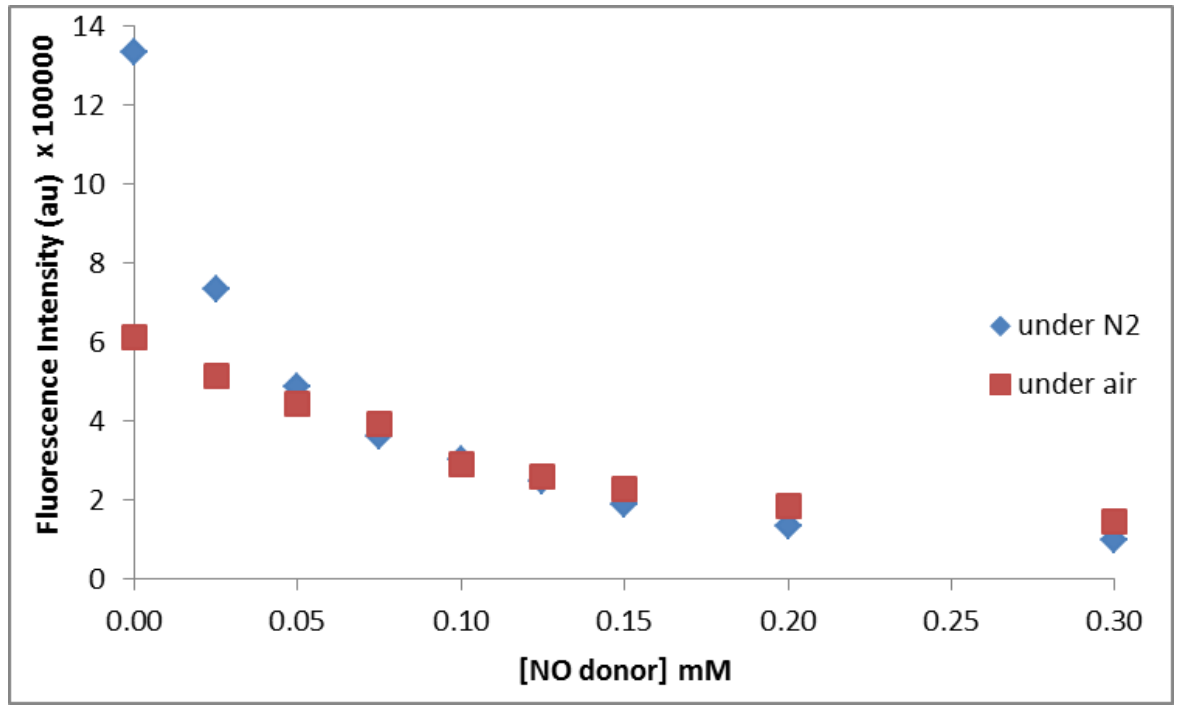

Figure 2.7 Fluorescence Titrations of $\mathbf{1}(10 \mu \mathrm{M})$ with NO-donor $(0-300 \mu \mathrm{M})$, under $\mathrm{N}_{2}$ vs. under air. I514nm vs. NO-donor concentration. 
The same comparison for titration of $\mathbf{1}$ with NO under anaerbic vs. aerobic conditions was carried out also after photochemical preparation of the complex. Under such conditions the ratiometricity in anaerobic vs. aerobic conditions can be compared. Complex 1 prepared photochemically on a $1 \mathrm{mg}$ scale (1.67 $\mathrm{mM}$ in THF) showed ratiometric response to $\mathrm{NO}$ addition at $560 \mathrm{~nm}$ (decrease) and at $455 \mathrm{~nm}$ (increase). The fluorescence spectrum of $\mathbf{1}$ in THF/acetone/buffer 2/23/75 shows one emission at 562 nm. The shift in emission compared to the isolated complex could be ascribed both to the difference in the solvent system, and the presence of unreacted (phen) $\mathrm{Re}(\mathrm{CO})_{3} \mathrm{SO}_{3} \mathrm{CF}_{3}$, or (phen)Re(CO) $)_{2}(\mathrm{THF}) \mathrm{SO}_{3} \mathrm{CF}_{3}$ which emit at $560 \mathrm{~nm}$ and $585 \mathrm{~nm}$ respectively (see Chapter 3). Figure 2.8 shows the comparison of fluorescence intensity ratio ( $\left.\mathrm{I}_{454} / \mathrm{I}_{562}\right)$ upon titration of 1 with NO, in the form of NO-donor MAHMA-NONOate (0-300 $\mu \mathrm{M})$ dissolved in $0.04 \mathrm{M} \mathrm{NaOH}$ in $25 \mu \mathrm{L}$ portions. The two plots in the graph compare the intensity ratios observed under nitrogen and under air. The ratiometric response under $\mathrm{N}_{2}$ appears more consistent and linear, suggesting that the Re-dye reacts directly with NO and not with oxidized products, such as $\mathrm{NO}_{2}$ or $\mathrm{N}_{2} \mathrm{O}_{3}$. 


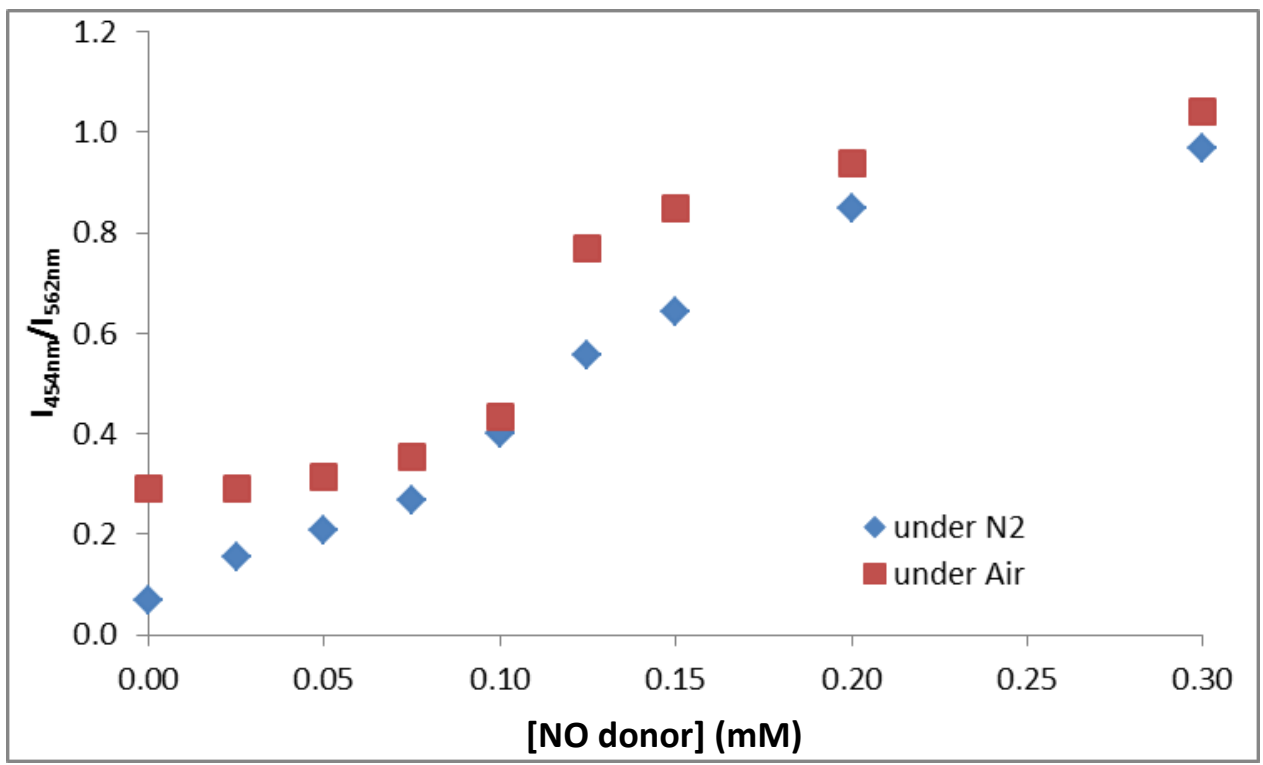

Figure 2.8 Graph of photochemically substituted (phen) $\mathrm{Re}\left(\mathrm{PPh}_{3}\right)(\mathrm{CO})_{2} \mathrm{OSO}_{2} \mathrm{CF}_{3}$ in THF $10 \mu \mathrm{M}$ with NO, acetone/buffer $25: 75, \lambda_{\text {exc }}=360 \mathrm{~nm}$, comparison nitrogen vs air

\subsubsection{Fluorescence titration with low concentrations of NO-donor.}

Figure 2.9 shows the fluorescence emission of $\mathbf{1}$ upon titration with $\mathrm{NO}$, in the form of NO-donor MAHMA-NONOate $(0-150 \mu \mathrm{M})$ dissolved in $0.04 \mathrm{M} \mathrm{NaOH}$, added in 1 - $25 \mu \mathrm{L}$ portions after $10 \mathrm{~min}$. intervals, under anaerobic conditions. The $514 \mathrm{~nm}$ decreases rapidly upon NO addition and emission at $426 \mathrm{~nm}$ increases correspondingly with NO-donor concentration. The $\mathrm{NaCl}$ concentration was lower than in the titration experiment described in 2.4.3 and this was found to reduce the intensity of the $514 \mathrm{~nm}$ emission, but not affecting the intensity of the emerging emission at $426 \mathrm{~nm}$. The experiment was performed under nitrogen atmosphere in order to avoid NO oxidation to $\mathrm{N}_{2} \mathrm{O}_{3}$. The observed response under anaerobic conditions implies that sensing is a direct result of a reaction of NO with the $\mathrm{Re}(\mathrm{I})$ dye. 


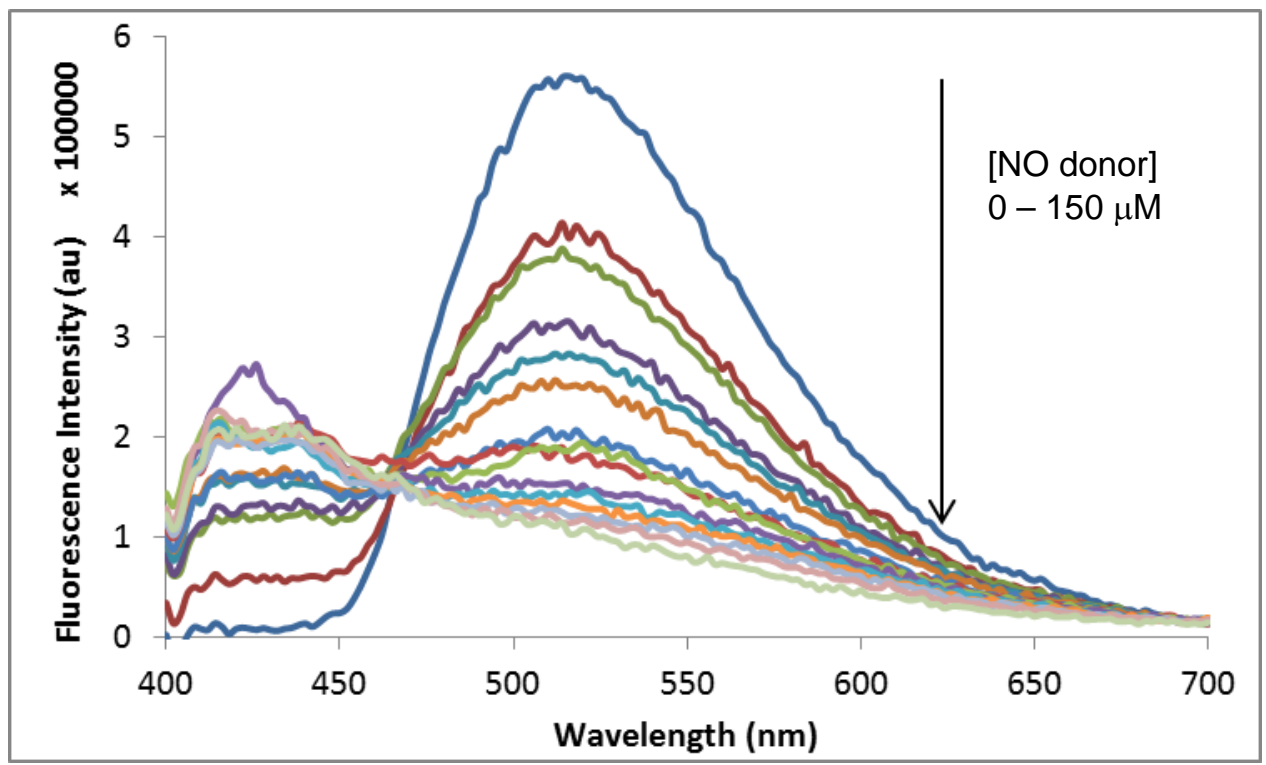

Figure 2.9 Fluorescence Spectra of $25 \mu \mathrm{M} 1$ (thermally prepared) with NO, acetone/buffer $25: 75, \lambda_{\text {exc }}=360 \mathrm{~nm}$, under nitrogen.

Figure 2.10 exhibits complex 1 prepared photochemically on a $1 \mathrm{mg}$ scale $(1.67$ $\mathrm{mM}$ in THF) showed ratiometric response to NO addition at $516 \mathrm{~nm}$ (decrease) and at $436 \mathrm{~nm}$ (increase).

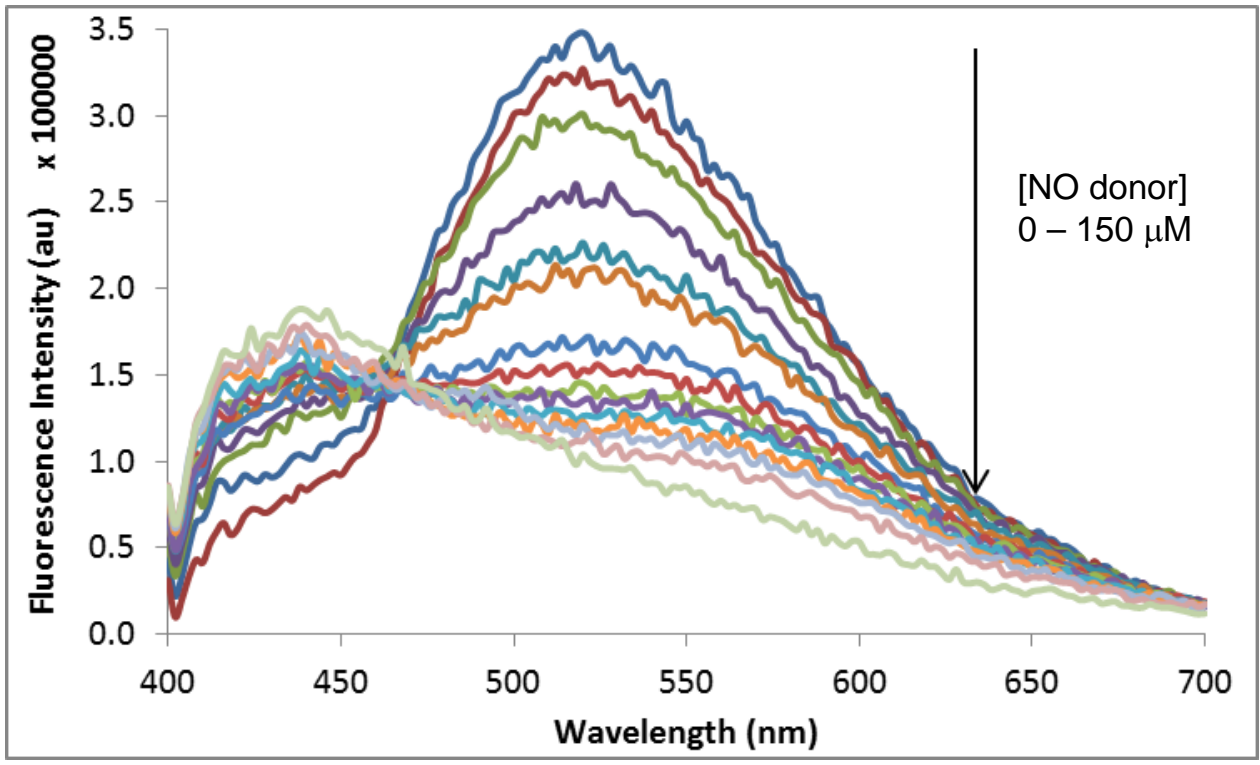

Figure 2.10 Fluorescence Spectra of $25 \mu \mathrm{M} 1$ (photochemically prepared) with NO, acetone/buffer $25: 75, \lambda_{\text {exc }}=360 \mathrm{~nm}$, under nitrogen. 
Figure 2.11 shows the change in fluorescence intensity with increasing NO-donor concentration. As exhibited in Figure 2.7, the intensity of the isolated (thermally produced) complex 1 is double than the photochemically produced mixture.

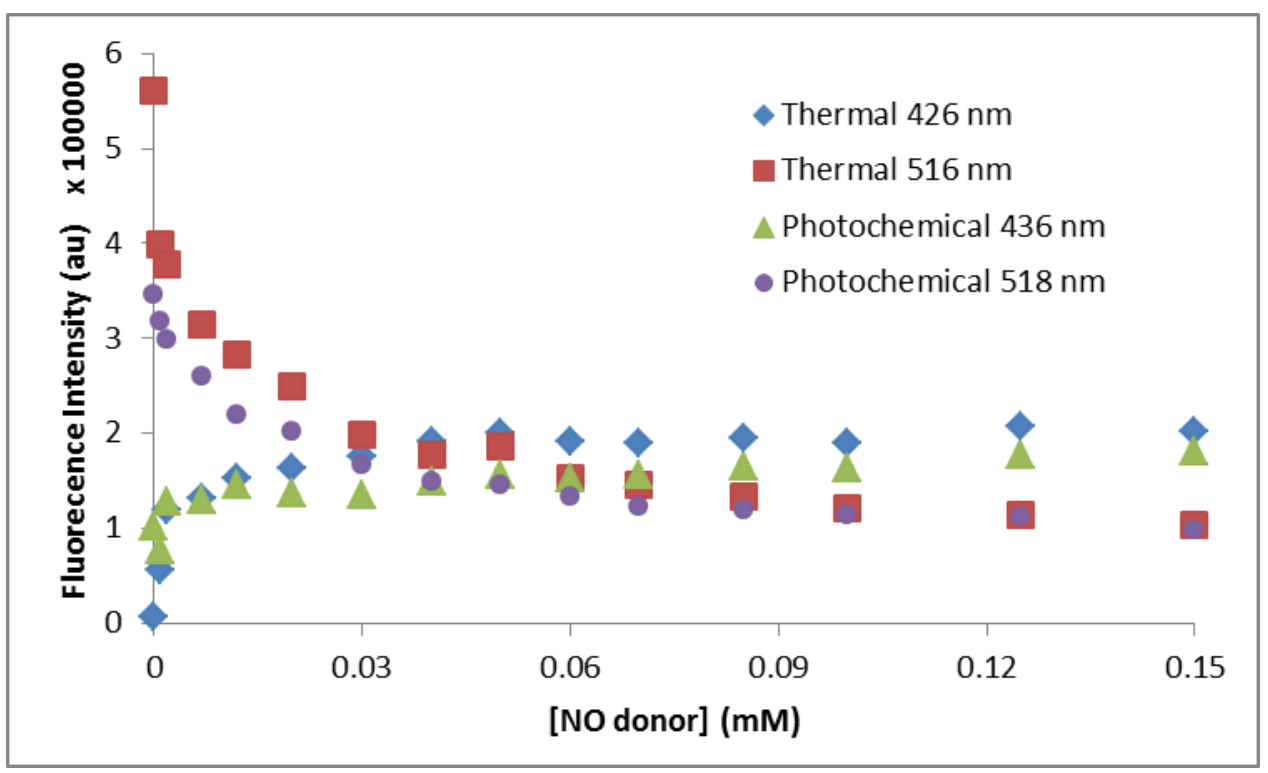

Figure 2.11 Observed fluorescence intensities of $25 \mu \mathrm{M} 1$ (thermally prepared) with gradual addition of NO-donor, acetone/buffer 25:75, $\lambda_{\text {exc }}=360 \mathrm{~nm}$

Figure 2.12 exhibits the comparison of fluorescence intensity ratios when the NOdonor concentration increases. The chosen signals were $426 \mathrm{~nm}$ and $515 \mathrm{~nm}$ for complex 1 prepared thermally; and $436 \mathrm{~nm}$ and $518 \mathrm{~nm}$ for complex 1 parepared photochemically. An important observation after analyzing the ratio of 1 prepared on either technique (photochemically or thermally) is the presence of two phenomena, higher response at lconcentrations lower than $5 \mu \mathrm{M}$ of NO-donor than at high ones. 


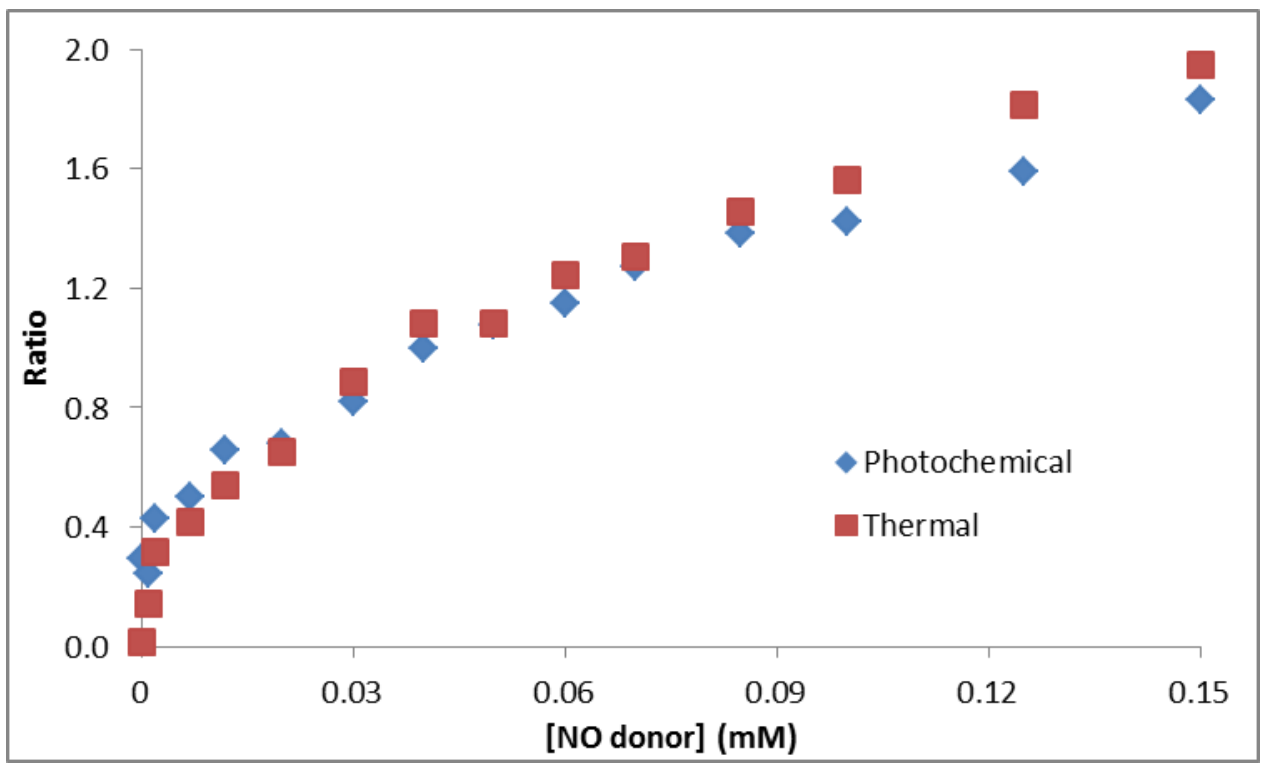

Figure 2.12 Observed fluorescence ratio of $25 \mu \mathrm{M} 1$ (photochemically and thermally prepared) with gradual addition of NO-donor, acetone/buffer 25:75, $\lambda_{\text {exc }}=360 \mathrm{~nm}$

\subsubsection{FT-IR spectroscopy:}

The FT-IR spectra were collected before (Fig. 2.9) and after the addition of NOdonor to (phen)Re(PPh$)(\mathrm{CO})_{2} \mathrm{OSO}_{2} \mathrm{CF}_{3}$. After adding $\mathrm{NO}$, the solution was let evaporate for 12 hours, for removing acetone, the solids settled down in the tube. The remaining solution was decanted to remove basic impurities, such as MAHMANONOate reaction products and uncoordinated phen and analogs, and the spectrum was collected (Fig. 3.10). In the spectrum of the product after NO addition bands were observed at $1739 \mathrm{~cm}^{-1}, 1660 \mathrm{~cm}^{-1}$ and $1633 \mathrm{~cm}^{-1}$, which are not present in the starting material and can be assigned to metal-nitrosyl complexes. These typically show stretching bands in the $1600 \mathrm{~cm}^{-1}-1750 \mathrm{~cm}^{-1}$ range. ${ }^{18}$ Nitroso compounds (organic NO) typically appear below $1600 \mathrm{~cm}^{-1}$. Even though NO stretching bands are typically strong they appear rather weak in our spectra, thus indicating that the Nitrosyl complex may not be the major product of the reaction of NO with the Re complex, nevertheless the 
presence of these bands, which do not appear when there is no reaction with NO, are indicative of a Re-NO complex. The typical triflate signals between $1122 \mathrm{~cm}^{-1}$ and 1267 $\mathrm{cm}^{-1}$ are missing in figure 2.10 .

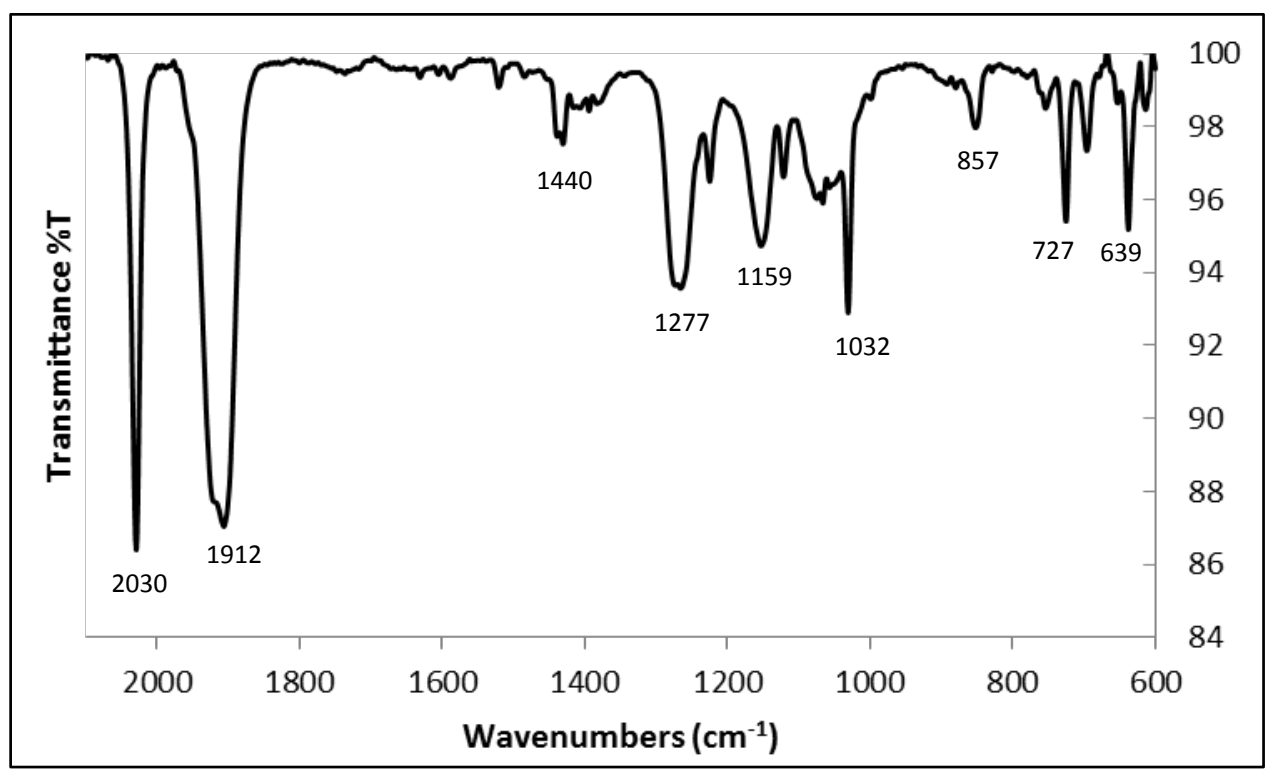

Figure 2.13 FT-IR spectrum of (phen) $\mathrm{Re}(\mathrm{CO})_{2}\left(\mathrm{PPh}_{3}\right) \mathrm{OSO}_{2} \mathrm{CF}_{3}(\mathbf{1})$

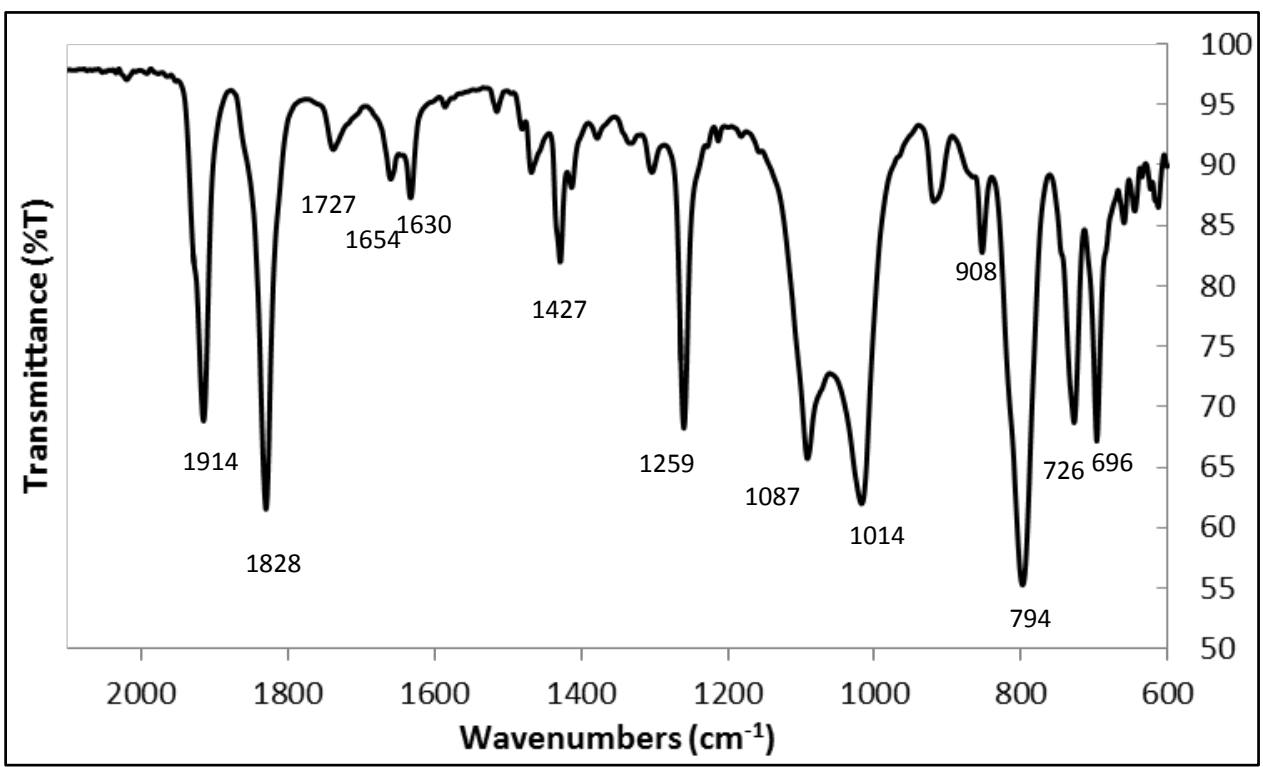

Figure 2.14 FT-IR spectrum of 2 after reaction with NO-donor. 


\subsection{6. ${ }^{1} \mathrm{H}-\mathrm{NMR}$ and ${ }^{19} \mathrm{~F}$-Study of Re complex reactivity:}

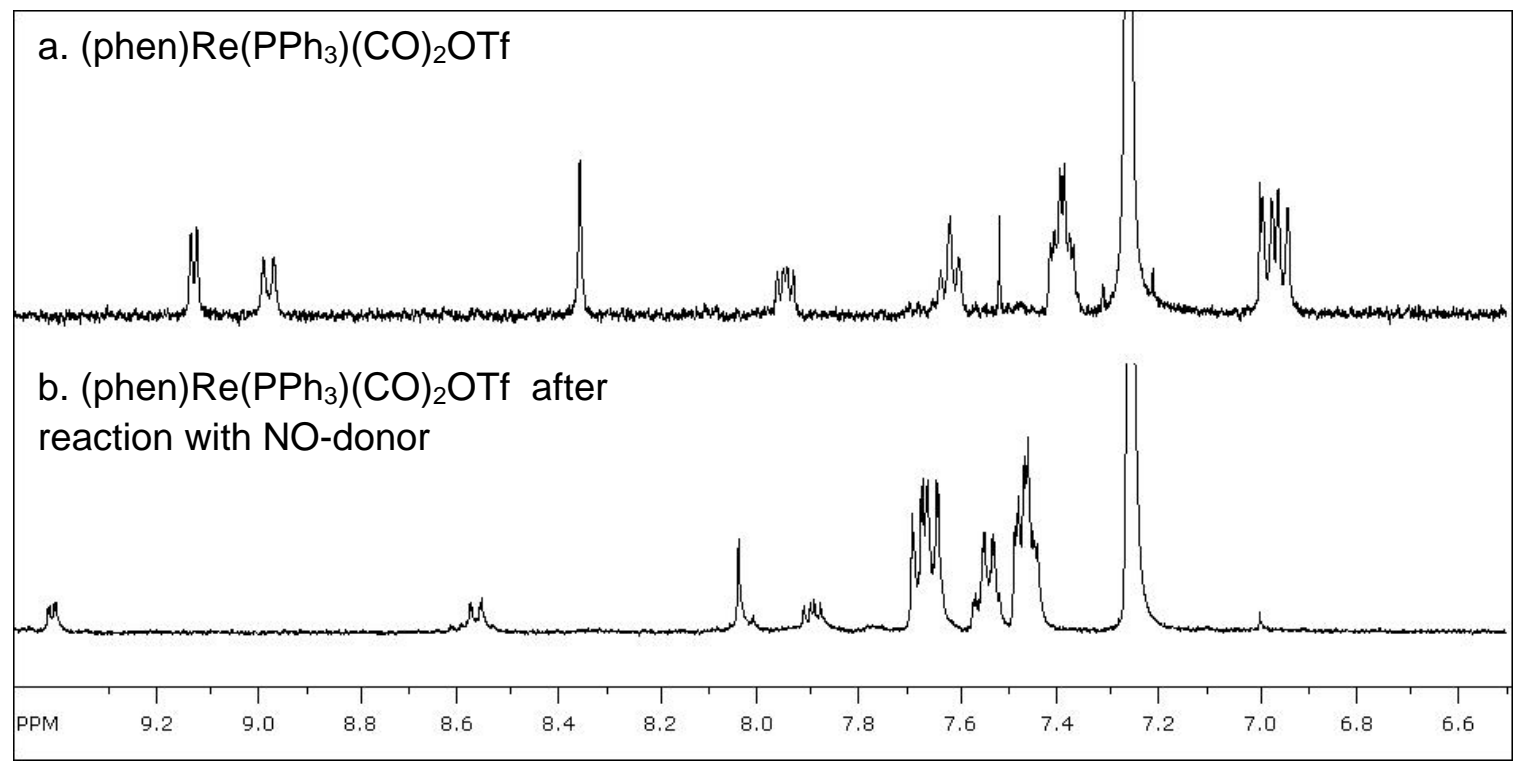

Figure 2.15 Aromatic region of NMR of $\mathbf{2}$ before (a) and after (b) reaction with NOdonor

Figure 2.15 shows the spectrum of (phen) $\mathrm{Re}\left(\mathrm{PPh}_{3}\right)(\mathrm{CO})_{2} \mathrm{OSO}_{2} \mathrm{CF}_{3}$ before (Fig. 2.15a) and after (Fig. 2.15b) reaction with NO-donor. Spectrum 2.15b clearly shows the resonances of free triphenylphosphine. Furthermore, experiments performed in identical conditions for control compounds (phen) $\operatorname{Re}(\mathrm{CO})_{3} \mathrm{Cl}$ and (phen) $\mathrm{Re}(\mathrm{CO})_{3} \mathrm{OTf}$ showed no changes after $\mathrm{HCl} / \mathrm{NaHCO}_{3}$ washing in the spectrum of (phen) $\mathrm{Re}(\mathrm{CO})_{3} \mathrm{Cl}$, while for (phen) $\mathrm{Re}(\mathrm{CO})_{3} \mathrm{OTf}$, resonances identical to (phen) $\mathrm{Re}(\mathrm{CO})_{3} \mathrm{Cl}$ were observed, presumably because of substitution of triflate with $\mathrm{Cl}^{-}$. These experiments underscore the need for a labile L-type ligand, and suggest that triphenylphosphine dissociates for NO can react with the metal center. ${ }^{19}$ F-NMR after NO-donor reaction showed no coordinated triflate signals at $-78.4 \mathrm{ppm}$, before or after acid wash. Thus, it can be concluded that $\mathrm{CF}_{3} \mathrm{SO}_{3}{ }^{-}$got dissociated after reaction with $\mathrm{NO}$, suggesting that $\mathrm{NO}$ 
displaces both triflate and $\mathrm{PPh}_{3}$ in order to coordinate to $\mathrm{Re}(\mathrm{I})$. Such mechanism is consistent with the rationale of using Re complexes containing labile $\mathrm{L}$ and $\mathrm{X}$ ligands for NO sensing.

\subsubsection{Cell Studies \& Fluorescence Microscopy:}

The solution results and the observed fluorescence responses upon NO addition to $\mathbf{1}$, suggested that these responses could also be observed in cells under aqueous media, $37 \mathrm{C}$ and neutral $\mathrm{pH}$. A first step towards applicability of this dye in living cells is to test its absorptivity and emission under physiological conditions. 1 was used as an initial model of absorptivity into Rat Aortic Endothelial Cells (RAOEC), since it could be readily prepared in a solid form and in dilute solutions.

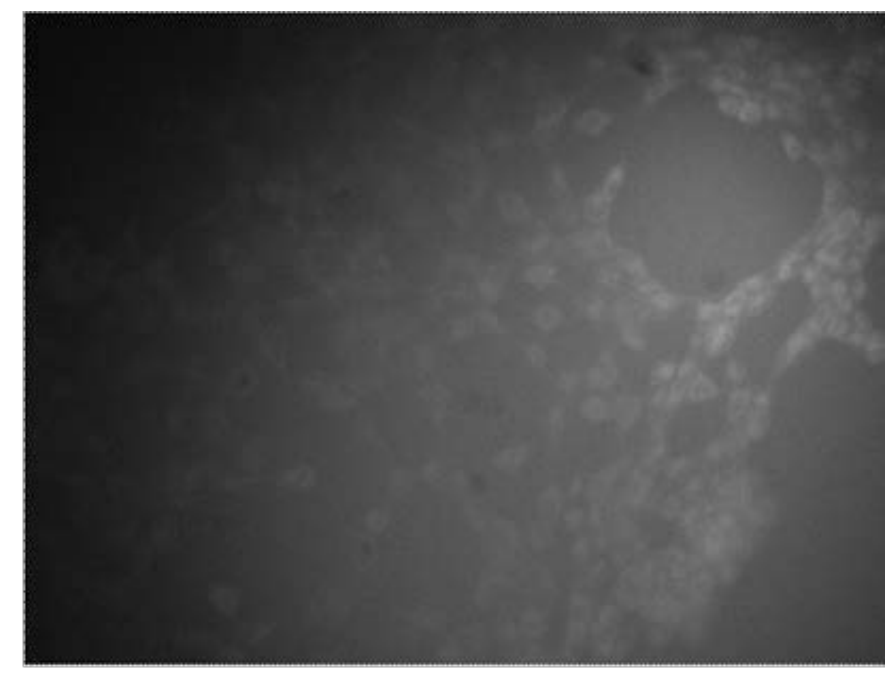

Figure 2.16 Fluorescence response of $2(10 \mu \mathrm{M})$ in RAOEC at $\lambda_{\mathrm{em}}=450 \mathrm{~nm}$ $\left(\lambda_{\text {exc }}=350 \mathrm{~nm}\right)$ showing the absorption of 2 into living cells.

Figure 2.16 shows that (phen) $\mathrm{Re}\left(\mathrm{PPh}_{3}\right)(\mathrm{CO})_{2} \mathrm{OSO}_{2} \mathrm{CF}_{3}$ demonstrated good absorptivity into cells via diffusion, in the same fashion that other fluorescent dyes. ${ }^{18}$

\subsection{CONCLUSION}


The complex (phen) $\mathrm{Re}\left(\mathrm{PPh}_{3}\right)(\mathrm{CO})_{2} \mathrm{OSO}_{2} \mathrm{CF}_{3}$ has been synthesized and characterized. This complex can react with NO under physiological conditions, producing a quenching in fluorescence that can be monitored. This complex has permeability through the cell membrane by diffusion and it can be used as fluorescent probe for NO oxide imaging. This is the first example of a rhenium phosphine complex used as an NO sensor.

\subsection{REFERENCES}

1. (a) Nagano, T.; Takizawa, H.; Hirobe, M. Tetrahedron Lett. 1995, 36, 8239; (b) Nakatsubo, N.; Kojima, H.; Kikuchi, K.; Nagoshi, H.; Hirata, Y.; Maeda, D.; Imai, Y.; Irimura, T.; Nagano, T. FEBS Lett. 1998, 427, 263; (c) Kojima, H.; Sakurai, K.; Kikuchi, K.; Kawahara, S.; Kirino, Y.; Nagoshi, H.; Hirata, Y.; Nagano, T. Chem. Pharm. Bull. 1998, 46, 373; (d) Nakatsubo, N.; Kojima, H.; Sakurai, K.; Kikuchi, K.; Nagoshi, H.; Hirata, Y.; Akaike, T.; Maeda, H.; Urano, Y.; Higuchi, T.; Nagano, T. Biol. Pharm. Bull. 1998, 21, 1247.

2. (a) Sun, W. C.; Gee, K. R.; Klaubert, d. H.; Haugland, R. P. J. Org. Chem. 1997, 62, 6469; (b) Tanaka, K.; Miura, T.; Umezawa, N.; Urano, Y.; Kikuchi, K.; Higuchi, T.; Nagano, T. J. Am. Chem. Soc. 2001, 123, 2530-2536; (c) Yamaguchi, K.; Tamura, Z.; Maeda, M. Acta Crystallogr. 1997, C53, 284.

3. Pluth, M.D.; Lippard, S.J. Chem. Commun., 2012, 48, 11981; (b) Lim, M. H,; Xu, D.; Lippard, S. J. Nat. Chem. Biol., 2006, 2, 375-380.

4. (a) Hollis, T. K.; Odenkirk, W.; Robinson, N. P.; Whelan, J.; Bosnich, B. Tetrahedron, 1993, 49, 5415; (b) Odenkirk, W.; Rheingold, A. L.; Bosnich, B. J. Am. Chem. Soc., 1992, 114, 6392; (c) Failer, J. W.; Ma, Y.; Smart, C. J.; DiVerdi, M. J. J. Organomet. Chem. 1991, 420, 237; (d) Failer, J. W.; Ma, Y. J. Am. Chem. Soc. 1991, 113, 1579; (e) Honeychuck, R. V.; Bonnesen, P. V.; Farahi, J.; Hersh, W. H.; J. Org. Chem. 1987, 52, 5296; (f) Bonnesen, P. V.; Puckett, C. L.; Honeychuck, R. V.; Hersh, W. H. J. Am. Chem. Soc. 1989, 111, 6070.

5. (a) Veghini, D.; Nefedov, S. E.; Schmalle, H.; Berke, H. J. Organomet. Chem. 1996, 526, 117-134; (b) Machura, B. Coord. Chem. Rev. 2005, 249, 2277-2307.

6. (a) Trovati, A.; Uguagliati, P. Inorg. Chem. 1971, 10, 851; (b) Wang, Y.; Frausto da Silva, J. J. R.; Pombeiro, A. J. L.; Pellinghelli, M. A.; Tiripicchio, A. J. Organomet. Chem., 1992, 430, C56; (c) Wang, Y.; Frausto da Silva, J. J. R.; Pombeiro, A. J. L.; Pellinghelli, M. A.; Tiripicchio, A. J. Organomet. Chem. 
1994, 476, C9.

7. (a) Batczak, T. J.; Czurak, W.; Dziegielewski, J. O.; Machura, B.; Jankowska, A.; Kusz, J.; Warczewski, J. J. Coord. Chem. 2001, 52, 361; (b) Machura, B.;

Kruszynski, R.; Jaworska, M. Polyhedron, 2005, 24, 419; (c) Cameron, T. S.;

Grundy, K. R.; Robertson, K. N. Inorg. Chem. 1982, 21, 4149; Chen, J. Y.;

Grundy, K. R.; Robertson, K. N. Can. J. Chem. 1989, 67, 1187.

8. (a) Lees, A. J. Chem. Rev. 1987, 87, 711-743; (b) Stufkens, D. J.; Vlček A. Jr. Coord. Chem. Rev. 1998, 177, 127-179; (c) Vlček Jr., A. Coord. Chem. Rev. 1998, 177, 219-256; (d) Vlcek A. Jr. Coord. Chem. Rev. 2000, 200, 933-977; (e) Striplin, D. R.; Crosby, G. A. Chem. Rev. 2001, 211, 163-175; (f) Kumar, A.; Sun, S.-S.; Lees, A. J. Top. Organomet. Chem. 2010, 29, 1-35; (g) Wrighton, M. S.; Morse, D. L. J. Am. Chem. Soc. 1974, 96, 998-1003; (h) Fredericks, S. M.; Luong, J. C.; Wrighton, M. S. J. Am. Chem. Soc. 1979, 101, 7415-7417; (i) Caspar, J. V.; Meyer, T. J. J. Phys. Chem. 1983, 87, 952-957; (j) Lees, A. J. Comments Inorg. Chem. 1995, 17, 319-346; (k) Baba, A. I.; Shaw, J. R.; Simon, J. A.; Thummel, R. H. Schmehl, Coord. Chem. Rev. 1998, 171, 43-59; (l) Wallace, D. C. Jackman, R. P.; Rillema, D. P.; Merkert, J. W. Inorg. Chem. 1995, 34, 5210-5214; (m) Dyer, J.; Blau, W. J.; Coates, C. G.; Creely, C. M.; Gavey, J. D.; George, M. W.; Grills, D. C.; Hudson, S.; Kelly, J. M.; Matousek, P.; McGarvey, J. J.; McMaster, J.; Parker, A. W.; Towrie, M.; Weinstein, J. A.; Photochem. Photobiol. Sci. 2003, 2, 542-554; (n) Kuimova, M. K.; Sun, Z. P.; Matousek, X.; Grills, D. C.; Parker, A. W.; Towrie, M.; George, M. W. Photochem. Photobiol. Sci. 2007, 6, 1158-1163.

9. (a) Fischer, M. J., Jelliss, P. A., Phifer, L. M., and Rath, N. P. Inorg. Chim. Acta 2005, 358, 1531-1544; (b) Fischer, M. J., Jelliss, P. A., Orlando, J. H., Phifer, L. M., and Rath, N. P. J. Lumin. 2005, 114, 60-64; (c) Glezen, M. M. and Lees, A. J. J. Am. Chem. Soc. 1988, 110, 3892-3897; (d) Stacy, N. E., Conner, K. A., McMillin, D. R., and Walton, R. A. Inorg. Chem. 1986, 25, 3649-3652; (e) Loukova, G. V., Huhn, W., Vasiliev, V. P., and Smirnov, V. A. J. Phys. Chem. A 2007, 111, 4117-4121; (f) Li, C.-K., Cheng, E. C.-C., Zhu, N., and Yam, V. W.W. Inorg. Chim. Acta 2005, 358, 4191-4200; (g) Yam, V. W. W., Lam, C. H., Fung, W. K. M., and Cheung, K. K. Inorg. Chem. 2001, 40, 3435-3442; (h) Kunkely, H. and Vogler, A. J. Photochem. Photobiol. A 2001, 146, 63-66; (i) Williams, D. S. and Korolev, A. V. Inorg. Chem. 1998, 37, 3809-3819.

10. Tsubaki, H., Sekine, A., Ohashi, Y., Koike, k., Takeda, H., Ishitami, O. J. Am. Chem. Soc., 2005, 127, 15544-15555

11. (a) Lee, Y. F. and Kirchhoff, J. R. J. Am. Chem. Soc. 1994, 116, 3599-3600; (b) Nakamaru, K. Bull. Chem. Soc. Jpn. 1982, 55, 2697-2705. 
12. (a) Geoffroy, G. L.; Wrighton, M. S. Organometallic Photochemistry; Academic Press: New York, 1979; (b) Lees, A. J. Chem. Rev. 1987, 87, 711-743.

13. Kalyanasundaram, K. Photochemistry of Polypyridine and Porphyrin Complexes; Academic Press: New York, 1992; Chap. 10, 321-329.

14. Choi, A. W. T.; Poon, C. S.; Liu, H. W.; Cheng, H. K.; Lo, K. New J. Chem., 2013, 37, 1711-1719.

15. Kavalllieratos, K; Tsoukias, N. M.; Lozano-Lewis, L. I. "Organometallic dyes for NO detection and imaging". US Patent 8,906,694B2. Granted: Dec. 9, 2014.

16. Tsubaki, H., Sekine, A., Ohashi, Y., Koike, k., Takeda, H., Ishitami, O. J. Am. Chem. Soc., 2005, 127, 15544-15555

17. (a) Pratihar, P.; Dasmahapatra, A. K.; Sinha, C. Polyhedron, 2007, 26, $1217-$ 1221. (b) Machura, B. Coordination Chemistry Reviews, 2005, 249, 2277-2307.

18. (a) Wright, K. M.; Oparka, K. J. Planta, 1989, 179, 257-64. (b) Achilefu, S. I.; Dorshow, R. B.; Bugaj, J. E.; Rajagopalan, R. Proceedings of the International Society for Optical Engineering. 2000, 3917, 80-86. (c) Zhu, H.; Fan, J.; Li, M.; Cao, J.; Wang, J.; Peng, X. Chem. Eur. J., 2014, 20, 4691-4696. 


\section{3. (phen)Re(THF)(CO) ${ }_{2} \mathrm{OSO}_{2} \mathrm{CF}_{3}$ AS A FLUORESCENT ORGANOMETALLIC COMPLEX NO SENSOR.}

\subsection{ABSTRACT}

The complex (phen)Re(THF)(CO) ${ }_{2} \mathrm{OSO}_{2} \mathrm{CF}_{3}$ (2) that can be obtained photochemically or thermally shows ratiometric response upon NO addition, which was added using the Z-1-N-methyl-N-[6-(N-methylammoniohexyl)amino]diazen-1-ium-1,2diolate (MAHMA-NONOate) as an NO-donor. Addition of 5-150 $\mu \mathrm{M}$ of NO-donor into a $20 \mu \mathrm{M}$ solution of (phen) $\mathrm{Re}(\mathrm{THF})(\mathrm{CO})_{2} \mathrm{OSO}_{2} \mathrm{CF}_{3}$ obtained thermally or photochemically from (phen) $\mathrm{Re}(\mathrm{CO})_{3} \mathrm{OSO}_{2} \mathrm{CF}_{3}$ in $25 \%$ v/v acetone/phosphate saline(PBS) buffer gave fluorescence quenching at 582nm and a concurrent increase in emission at 450nm, upon excitation at 360nm. The FT-IR spectroscopy spectrum, upon addition of NO-donor, showed bands at $1720 \mathrm{~cm}^{-1}$ and $1631 \mathrm{~cm}^{-1}$ that can be ascribed to nitrosyl complexes formed upon reaction with NO. Nuclear Magnetic Resonance (NMR) studies have shown that the NO fluorescence responses can be ascribed to the reactivity of the NO with the organometallic complex. Fluorescence microscopy studies on Rat Aortic Endothelial Cells in 2\% v/v acetone/phosphate saline (PBS) buffer using $1 \mu \mathrm{M}$ of 2, and 1-10 $\mu \mathrm{M}$ of NO-donor gave notable increases in fluorescence emission at $450 \mathrm{~nm}$ $\left(\lambda_{\text {exc }}=350 \mathrm{~nm}\right)$ and distinct incorporation of the dye into the cell. The system demonstrates the potential of a new approach for the design of fluorescent NO sensors based on the reactivity and fluorescence changes upon NO coordination to third-row transition-metal organometallic fluorescent complexes. 


\subsection{INTRODUCTION}

Nitric oxide has several functions of particular importance for human health. ${ }^{1,2}$ It is now recognized as a key signaling molecule in the cardiovascular system because it regulates blood pressure via vasodilation. ${ }^{3}$ Despite numerous significant scientific contributions recently on NO physiology and biomedical functions, ${ }^{4}$ fundamental questions about basic physiological functions of NO remain unclear., ${ }^{5,6}$ Over the last decade, there have been several efforts to model NO-dependent signaling in the cardiovascular system ${ }^{7,8,9,10}$ and controversies regarding the role of $\mathrm{NO}$ in the vasculature have been discussed. ${ }^{11}$ Thus, effective quantification and detection of NO in biological tissues is of paramount importance in understanding its role and physiology.

Quantification and imaging of NO in tissues remains a challenge because of the absence of a detection method that can combine significant spatial and temporal resolution with high $\mathrm{NO}$ selectivity ${ }^{12}$. Challenges lie in NO's short lifetime $\left(\mathrm{t}_{1 / 2}=c a .5\right.$ s) and the fact that it is found in low concentrations in living tissues. ${ }^{13}$

The NO-sensitive fluorescent dyes, such as diaminofluorescein, DAF-FM ${ }^{14}$ and Copper-Fluorescein $\mathrm{Cu}-\mathrm{FL},{ }^{15}$ can provide practical methods for quantification and imaging of NO in vitro and in vivo. Some recent studies ${ }^{16}$ have reported fluorescent probes using different chemical concepts, including what is claimed to be the first ratiometric fluorescent probe for NO. ${ }^{16 \mathrm{c}}$ In a recent report a combined rhodaminecoumarin fluorophore showed ratiometric sensing for NO, with an fluorescence emission increase at $583 \mathrm{~nm}$ and a decrease at $473 \mathrm{~nm}$ even in cells.

2,2'-bipy and phenanthroline complexes of $\operatorname{Re}(\mathrm{I})$ and $\mathrm{Ru}(\mathrm{II})$ have been used as

fluorescent platforms, and in photoluminescence applications. ${ }^{17}$ Their favorable 
photochemical properties, stability of NO complexes,${ }^{18}$ and flexibility in accommodating conjugated ligand systems offer potent scaffolds for the design of novel NO-selective dyes. Recently Choi and $\mathrm{Lo}^{19}$ have incorporated a diaminopyridine into a $\operatorname{Re}(\mathrm{phen})$ system to achieve sensing for NO, via the formation of a triazole derivative in the same fashion as it has been proposed for the DAF fluorophore reactivity with NO (via initial $\mathrm{N}_{2} \mathrm{O}_{3}$ formation). They observed an increase of fluorescence emission at $560 \mathrm{~nm}$, yet this Re sensor was not found to be responsive at HeLa cells upon NOC-7 NO-donor addition. Mascharak and Fry synthesized and tuned ruthenium nitrosyls complexes for targeted NO delivery to cells to induce apoptosis. ${ }^{20}$

As part of our efforts to explore new mechanisms of NO sensing using fluorescent organometallics, we are now demonstrating a concept of NO sensing based on NO coordination to a $\operatorname{Re}(\mathrm{I})$ center, resulting in a ratiometric response, upon NO addition in the form of the NO-donor MAHMA-NONOate. The ratiometric response is dependent on NO concentration, and is observed at $\mathrm{pH} 7.2$ in solution (25\% v/v acetone/PBS buffer) and also in Rat Aortic Endothelial Cells (2\% v/v acetone/PBS), by fluorescence microscopy. The FT-IR and NMR spectra were consistent with the formation of nitrosyl complexes upon reaction of the Re(I)-THF-complex that may be responsible for the observed changes in fluorescence. These new complexes provide a novel concept for Nitric Oxide detection in biological tissues that is based on a radically different concept that takes advantage of the photophysical properties and synthetic versatility and reactivity of the Re(phen)system to give a ratiometric organometallic NO sensor. To our knowledge this is the first example of a ratiometric NO sensor that uses coordination of NO to a metal center, without a redox reaction at the metal center, and taking advantage 
of the photophysical properties of phenanthroline complexes of third row transition metal organometallics.

In prior work (Chapter 2), it was shown that (phen) $\mathrm{Re}\left(\mathrm{PPh}_{3}\right)(\mathrm{CO})_{2} \mathrm{OSO}_{2} \mathrm{CF}_{3}$, prepared thermally or photochemically from (phen) $\mathrm{Re}(\mathrm{CO})_{3} \mathrm{OSO}_{2} \mathrm{CF}_{3}$ has the ability to react with NO under physiological conditions, producing a fluorescence change at 514 $\mathrm{nm}$ and it has cell membrane permeability.

\subsection{EXPERIMENTAL SECTION}

\subsubsection{Material and Methods}

(phen) $\mathrm{Re}(\mathrm{CO})_{3} \mathrm{Cl}$ and (phen) $\mathrm{Re}(\mathrm{CO})_{3} \mathrm{OSO}_{2} \mathrm{CF}_{3}$ were prepared as previously reported and were found spectroscopically identical to the reported compounds. ${ }^{21,22}$ All other materials (purchased from Aldrich Chemical Co., ACROS organics, Cayman Chemicals, or Alfa Aesar) were standard reagent grade and were used without further purification, unless otherwise mentioned. Tetrahydrofuran (THF) used for the preparation of 2, was anhydrous (purchased as an Aldrich Sure-Seal ${ }^{\mathrm{TM}}$ product), or distilled and dried before use. PBS buffer $(0.002 \mathrm{M}$ in total phosphate, $\mathrm{pH}=7.2$, $[\mathrm{NaCl}]=0.04 \mathrm{M}$ ) was prepared from $\mathrm{K}_{2} \mathrm{HPO}_{4}, \mathrm{KH}_{2} \mathrm{PO}_{4}$, and $\mathrm{NaCl}$ (all from Fisher Scientific). Water used for preparation of the buffers and experiments with Nitric oxide was filtered water $\left(18 \mathrm{M} \Omega \cdot \mathrm{cm}^{-1}\right)$ produced from a Millipore ${ }^{\circledR}$ nanopure diamond lab water system (Barnstead Thermolyne Corporation, Dubuque, IA). Deuterated solvents were purchased from Cambridge Isotope Laboratories Ltd. Rat Aortic Endothelial Cells acquired from Cell Biologics were used for the cell based experiments. Fetal Bovine Serum (FBS) and DMSO were bought from ATCC. Penicillin-Streptomycin and endothelial cell growth supplement from bovine neural tissue were bought from Sigma 
Aldrich. DMEM/F-12 medium, Phosphate Buffer Saline (PBS) and Trypsin (0.05\% EDTA) were bought from Invitrogen Inc. Cell culture flasks and dishes were purchased from Fisher Scientific. Photochemical reactions were carried out using a Rayonet photochemical reactor (Southern New England Ultraviolet, model RPR-100), equipped with a cooling fan and 10 phosphor coated low-pressure mercury lamps $\left(\lambda_{\max }=300 \mathrm{~nm}\right.$, light intensity $=5.2 \pm 0.1 \times 106$ photon $\left./ \mathrm{sec} / \mathrm{cm}^{3}\right)$. Fluorescence Spectra were recorded on a Jobin Yvon Horiba Fluoromax 3 spectrometer. Fluorescence Microscopy was performed using an Olympus IX 81 Fluorescence Microscope and analyzed with IPLab v 4.04 Scientific Imaging Processing Software. Frequency-domain fluorescence lifetime measurements were performed using a ChronosFD spectrofluorometer (ISS, Champaign, IL). Samples were excited with a $370 \mathrm{~nm}$ modulated diode, and emission was collected using $400 \mathrm{~nm}$ long-pass filters (Andover, Salem, NH). The ${ }^{1} \mathrm{H}$ and ${ }^{13} \mathrm{C}$ NMR spectra were recorded on a 400 Bruker NMR spectrometer and were referenced, using the residual solvent resonances. All chemical shifts, $\delta$, are reported in ppm. The FT-IR spectra were recorded with a Perkin Elmer Spectrum 100 FT-IR Spectrometer.

\section{$\begin{array}{llll}\text { 3.3.2. Conversion of } & \text { (phen) } \mathrm{Re}(\mathrm{CO})_{3} \mathrm{OSO}_{2} \mathrm{CF}_{3} & \text { to }\end{array}$} (phen)Re(CO) $)_{2}(\mathrm{THF}) \mathrm{OSO}_{2} \mathrm{CF}_{3}$

\subsubsection{Method 1 - Thermal Substitution:}

(phen) $\mathrm{Re}(\mathrm{CO})_{3} \mathrm{OSO}_{2} \mathrm{CF}_{3}(1 \mathrm{mg}, 1.67 \mu \mathrm{mol})$ and $1.00 \mathrm{~mL}$ of anhydrous tetrahydrofurane (final concentration $1.67 \mathrm{mM}$ ) were placed in a $10 \mathrm{~mL}$ round bottom flask, under $\mathrm{N}_{2}$ flow. The solution was refluxed for $48 \mathrm{~h}$. After refluxing, the color of the solution changed from yellow to amber. This solution was used without further treatment. 


\subsubsection{Method 2 - Photochemical Substitution:}

(phen) $\mathrm{Re}(\mathrm{CO}){ }_{3} \mathrm{OSO}_{2} \mathrm{CF}_{3}(1 \mathrm{mg}, 1.67 \mu \mathrm{mol})$ and $1.00 \mathrm{~mL}$ of anhydrous tetrahydrofurane (final concentration $1.67 \mathrm{mM}$ ) were placed in a NMR tube. The solution was irradiated with a Rayonet photochemical reactor (Southern New England Ultraviolet, model RPR-100), equipped with a cooling fan and 10 phosphor coated low-pressure mercury lamps $\left(\lambda_{\max }=300 \mathrm{~nm}\right.$, light intensity $\left.=5.2 \pm 0.1 \times 106 \mathrm{photon} / \mathrm{sec} / \mathrm{cm}^{3}\right)$. After irradiation the color of the solution changed from yellow to amber. This solution was used without further treatment. When this procedure was performed in $\mathrm{d}_{8}-\mathrm{THF}$, a set of signals different from the starting material were observed. ${ }^{1} \mathrm{H}-\mathrm{NMR}\left(\mathrm{THF}-\mathrm{d}_{8}\right): 7.98$ (d, $\left.2 \mathrm{H},{ }^{3} \mathrm{~J}_{\mathrm{H}-\mathrm{H}}=8.2 \mathrm{~Hz},{ }^{3} \mathrm{~J}_{\mathrm{H}-\mathrm{H}}=5.1 \mathrm{~Hz}\right), 8.36(\mathrm{~s}, 2 \mathrm{H}), 8.99\left(\mathrm{~d}, 2 \mathrm{H},{ }^{3} \mathrm{~J}_{\mathrm{H}-\mathrm{H}}=8.28 \mathrm{~Hz},{ }^{4} \mathrm{~J}_{\mathrm{H}-\mathrm{H}}=1.4\right.$ $\mathrm{Hz}), 9.14\left(\mathrm{~d}, 2 \mathrm{H},{ }^{3} \mathrm{~J}_{\mathrm{H}-\mathrm{H}}=4.7 \mathrm{~Hz},{ }^{4} \mathrm{~J}_{\mathrm{H}-\mathrm{H}}=1.4 \mathrm{~Hz}\right) .{ }^{19} \mathrm{~F}-\left\{{ }^{1} \mathrm{H}\right\}-\mathrm{NMR}\left(\mathrm{CDCl}_{3}\right):-78.1 \mathrm{ppm}$. FT-IR $\left(\mathrm{cm}^{-1}\right)$ : 2033 (vco, vs), 1910 (vco, vs), 1430, 1290, 1246, 1170, 1031, 848, 725, 637. UV-Vis (in ethanol): $\lambda_{\max }=360 \mathrm{~nm}-$ br $\left(\varepsilon=4500 \mathrm{~mol}^{-1} \mathrm{~cm}^{-1}\right)$, Fluorescence emission in acetone $\left(\lambda_{\text {exc }}=360 \mathrm{~nm}\right): 585 \mathrm{~nm} \Phi_{1}=0.002$ (at $370 \mathrm{~nm}$, in 1:3 acetone:PBS, using quinine sulfate in sulfuric acid $0.1 \mathrm{M}$ as reference $\left.\Phi_{\mathrm{R}}=0.546\right)$. Lifetime: $\tau_{1}=2.26$ $\pm 0.6 \mathrm{~ns}, \quad \mathrm{f}_{1}=0.114 \pm 0.005, \alpha_{1}=0.0504 ; \tau_{2}=41.3 \pm 1 \mathrm{~ns}, \mathrm{f}_{2}=0.457 \pm 0.01, \alpha_{2}$ $=0.0111 ; \tau_{3}=144 \pm 6 \mathrm{~ns}, \mathrm{f}_{3}=0.436 \pm 0.01, \alpha_{3}=0.00302$. The presence of 3 lifetimes symbolizes three different processes that happen at the same time. There is an ultrafast reaction, it may be a rapid exchange solvent substitution (substitution of THF by water), leading to inactivation. 


\subsubsection{Fluorescence studies with addition of NO-donor}

In a typical experiment a THF solution of Re complex prepared as per 2.3.2.1 or 2.3.2.2. (24 $\mu \mathrm{L}, 1.67 \mathrm{mM})$ is added to $970 \mu \mathrm{L}$ of acetone to result in $1.000 \mathrm{~mL}$ of a 100 $\mu \mathrm{M}$ solution in $97: 3 \mathrm{v} / \mathrm{v}$ acetone:THF. A quantity of $2.75 \mathrm{~mL}$ to $3.00 \mathrm{~mL}$ of aqueous PBS buffer ( $\mathrm{pH}=7.2$ ) was added. Then 0 to $150 \mu \mathrm{L}$ of $4 \mathrm{mM}$ stock solution of MAHMANONOate NO-donor, in $0.04 \mathrm{M} \mathrm{NaOH}$ were added, in order to obtain $75 \% \mathrm{v} / \mathrm{v}$ aqueous/organic solution and a final NO-donor concentration from 0 to $150 \mu \mathrm{M}$ range, while to concentration of the Re complex remains constant at $20 \mu \mathrm{M}$. No more than 10 minutes elapsed from preparation of the organic phase to the addition of NO-donor. The solution was mixed thoroughly in a vortex and sonicated for $90 \mathrm{~min}$ at room temperature. The fluorescence of all solutions was measured at $\lambda_{\text {exc }}=360 \mathrm{~nm}$ and recorded from 400 $\mathrm{nm}$ to $700 \mathrm{~nm}$. For a control experiment a $\mathrm{NaOH}$ stock solution was used instead of an NO-donor $/ \mathrm{NaOH}$ stock, in order to account for any possible variations in the fluorescence due to $\mathrm{pH}$ changes. The result of the control titrations gave identical spectra for all titration points for the $1-150 \mu \mathrm{L}$ range.

\subsection{4. ${ }^{1} \mathrm{H}-\mathrm{NMR}$ and ${ }^{19} \mathrm{~F}-\mathrm{NMR}$ study of reactivity of Re complexes with NO:} (phen)Re(THF)(CO) $)_{2} \mathrm{OSO}_{2} \mathrm{CF}_{3} \quad(1 \mathrm{mg}), \quad($ phen $) \mathrm{Re}\left(\mathrm{PPh}_{3}\right)(\mathrm{CO})_{2} \mathrm{OSO}_{2} \mathrm{CF}_{3} \quad(1$

$\mathrm{mg}){ }^{23}$ (phen) $\mathrm{Re}(\mathrm{CO})_{3} \mathrm{Cl}(1 \mathrm{mg})$, or (phen) $\mathrm{Re}(\mathrm{CO})_{3} \mathrm{OSO}_{2} \mathrm{CF}_{3}$ (1 mg) were added to 0.05 $\mathrm{mL}$ of THF in four separate $10 \mathrm{~mL}$ vials. $1.6 \mathrm{~mL}$ of acetone was added. Then $2.4 \mathrm{~mL}$ of PBS aqueous buffer $(\mathrm{pH}=7.2)$ and $0.2 \mathrm{~mL}$ of stock NO-donor solution $5 \mathrm{mM}$ in $0.04 \mathrm{M}$ $\mathrm{NaOH}$ were added sequentially. The mixtures were vortexed for $30 \mathrm{sec}$ and then sonicated for $3 \mathrm{~h}$ at room temperature. Dichloromethane was added $(3 \mathrm{x} 10 \mathrm{~mL})$, and the 
organic layer was dried with anhydrous sodium sulfate. The solvent was evaporated and the non-volatiles were taken up in chloroform and the NMR spectra were collected. In order to remove uncomplexed phenanthroline products and collect only the NMR of complexes the organic phases were further diluted with dichloromethane and washed with $1 \mathrm{M}$ hydrochloric acid, followed by $1 \mathrm{M}$ sodium bicarbonate, rinsed with deionized water, and dried with anhydrous sodium sulfate. The volatiles were evaporated, the solid residues were redissolved in chloroform-d, and the NMR spectra were collected. These experiments were also performed under identical conditions for control compounds (phen) $\mathrm{Re}(\mathrm{CO})_{3} \mathrm{Cl}$ and (phen) $\mathrm{Re}(\mathrm{CO})_{3} \mathrm{OTf}$. After $\mathrm{HCl} / \mathrm{NaHCO}_{3}$ washing no changes were

observed in the ${ }^{1} \mathrm{H}-\mathrm{NMR}$ spectrum of (phen) $\mathrm{Re}(\mathrm{CO}){ }_{3} \mathrm{Cl}$, while for (phen) $\mathrm{Re}(\mathrm{CO}){ }_{3} \mathrm{OTf}$, resonances identical to (phen) $\mathrm{Re}(\mathrm{CO})_{3} \mathrm{Cl}$ were observed.

3.3.5. Fluorescence microscopy studies of addition of NO-donor to (phen)Re(THF)(CO) ${ }_{2} \mathrm{OSO}_{2} \mathrm{CF}_{3}$ in Rat Aortic Endothelial Cells:

Cell preparation: Rat Aortic Endothelial Cells were grown in Dublecco's Modified Eagle Medium: Nutrient Mixture F-12 medium (DMEM/F-12) supplemented with 10\% Fetal Bovine Serum (FBS), 1\% penicillin-streptomycin, and endothelial cell growth supplement in a humidified tissue culture incubator at $37^{\circ} \mathrm{C}$ equilibrated with $95 / 5 \mathrm{v} / \mathrm{v} \%$ Air/ $/ \mathrm{CO}_{2}$ mixture. Cells were cultured in $35 \times 10 \mathrm{~mm}$ dishes seeded with $2 \times 10^{5}$ cells and used at $60-80 \%$ confluency.

Solution Preparation: A $500 \mu \mathrm{M}$ solution of $\mathrm{Re}(\mathrm{phen})(\mathrm{THF})(\mathrm{CO})_{2} \mathrm{OSO}_{3} \mathrm{CF}_{3}$ in THF/acetone (Solution B) was prepared from $62.5 \mu \mathrm{L}$ of a stock solution of $\mathrm{Re}\left(\right.$ phen)(THF)(CO) ${ }_{2} \mathrm{OSO}_{3} \mathrm{CF}_{3}$ in THF $\left(1.6 \times 10^{-3} \mathrm{M}\right.$ - Solution A) and $138 \mu \mathrm{L}$ of acetone. This solution was added to $800 \mu \mathrm{L}$ of $10 \mathrm{mM}$ PBS buffer solution ( $\mathrm{pH}=7.2)$, 
resulting in a final $100 \mu \mathrm{M}$ solution of $\mathbf{1}$ in 80/14/6 v/v aqueous buffer/acetone/THF. This new solution was further diluted 1/10 v/v with PBS Buffer before adding it to the cells, resulting in a final solution of $10 \mu \mathrm{M}$ of $\mathbf{1}$ in $98 / 1.4 / 0.6 \mathrm{v} / \mathrm{v}$ aqueous $\mathrm{PBS}$ buffer/acetone/THF (Solution C). Various quantities of $6 \mathrm{mM}$ NO-donor solution (MAHMA-NONOate) in $0.01 \mathrm{M} \mathrm{NaOH}$ were diluted with $0.01 \mathrm{M} \mathrm{NaOH}$ to give 0,100 , $500 \& 1000 \mu \mathrm{M}$ solutions of NO-donor (Solutions D-G).

Loading of solution $C(10 \mu M$ of Re complex 1): The old media was poured out from the plates; then the plates were rinsed twice with $1 \mathrm{~mL}$ of $10 \mathrm{mM} \mathrm{PBS}(\mathrm{pH}=7.2)$. Then 100 $\mu \mathrm{L}$ of DMEM/FBS/Penicillin Media, $800 \mu \mathrm{L}$ of PBS $10 \mathrm{mM}$, and $100 \mu \mathrm{L}$ of Re complex stock (Solution C) were added with this specific order. The plates were incubated for 75 min. at $37^{\circ} \mathrm{C}$ equilibrated with $95 / 5 \mathrm{v} / \mathrm{v} \%$ Air/ $/ \mathrm{CO}_{2}$ mixture.

Loading of NO-donor: First the reactive media was poured out from the plate; then the plates were rinsed twice with $1 \mathrm{~mL}$ of $10 \mathrm{mM}$ PBS $(\mathrm{pH}=7.2)$. Then $100 \mu \mathrm{L}$ of DMEM/FBS/Penicillin Media, $890 \mu \mathrm{L}$ of PBS $10 \mathrm{mM}$, and $10 \mu \mathrm{L}$ of NO-donor solution in $\mathrm{NaOH}$ from $0-1000 \mu \mathrm{M}$ range (Solutions $\mathrm{D}-\mathrm{G}$ ) were added to give final NO-donor concentrations of $0 \mu \mathrm{M}, 1 \mu \mathrm{M}, 5 \mu \mathrm{M}$, and $10 \mu \mathrm{M}$. The plates were incubated for $15 \mathrm{~min}$; the reactive media was poured out from the plate, and $1 \mathrm{~mL}$ of fresh PBS $10 \mathrm{mM}$ was added to each plate.

Fluorescence Microscopy Measurements: Each plate was placed in the fluorescence microscope and measured under a filter an excitation wavelength of $350 \mathrm{~nm}$ (330-380 nm window) and an emission window of 440-460 nm. The images were collected with a total exposition time of $2668 \mathrm{~ms}$ and 20X zoom. 


\subsection{RESULTS AND DISCUSSION}

\subsubsection{Design Considerations}

To achieve our goal of obtaining a ratiometric fluorescent probe that can detect changes in NO concentration with high sensitivity and selectivity, we are using an entirely new approach for NO sensing that combines the following elements: 1) NO binding to a $\operatorname{Re}(\mathrm{I})$ metal center, 2) a phenanthroline, attached on the metal, which provides favorable photophysical characteristics, ${ }^{17}$ as well potential for modification, for fine-tuning the emission wavelength, and 3) a labile ligand (THF or $\mathrm{OSO}_{2} \mathrm{CF}_{3}$ ) which is expected to be displaced by NO altering the fluorescence emission upon NO addition. NO coordination to the Re center is expected to shift the MLCT band to higher frequency, since NO is a $\pi$-acceptor, unlike THF that is a donor.

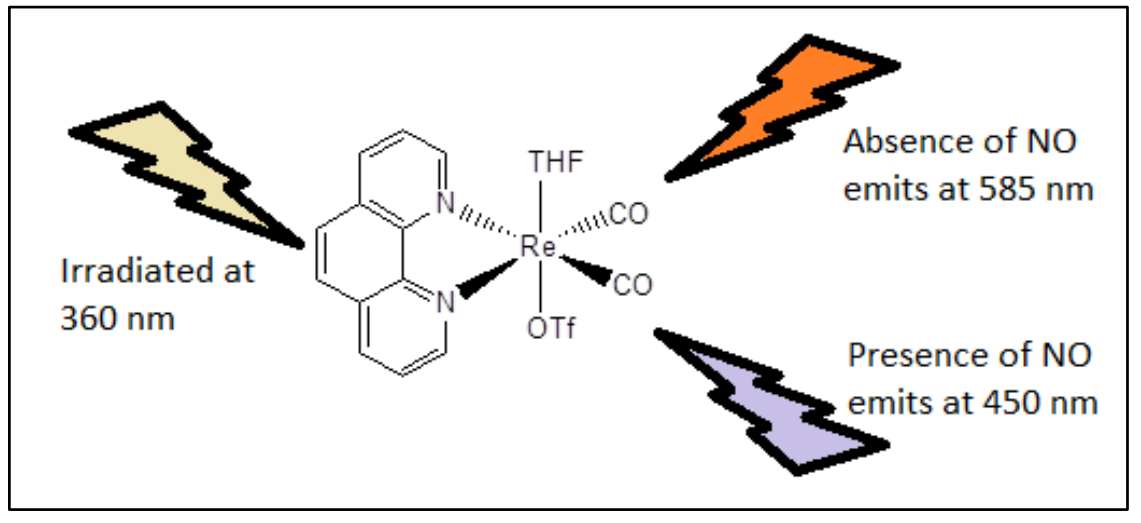

Scheme 3.1 Concept diagram for phenanthroline-rhenium NO sensors

\subsubsection{Fluorescence Studies:}

Solutions of (phen)Re(THF)(CO) ${ }_{2} \mathrm{OSO}_{3} \mathrm{CF}_{3}$ prepared photochemically or thermally were let to react with NO-donor in PBS buffer, without prior isolation of these species. Absorption Spectra of (phen)Re(THF)(CO) ${ }_{2} \mathrm{OSO}_{2} \mathrm{CF}_{3}$ in methanol and 
fluorescence spectra of solutions of (phen)Re(THF)(CO) ${ }_{2} \mathrm{OSO}_{2} \mathrm{CF}_{3}$ in acetone show characteristic differences from the spectra of the triflate complex with a single intense fluorescence emission at $585 \mathrm{~nm}$. However in the presence of water, such as in PBS buffer before addition of NO-donor, there is also a weak emission at $435 \mathrm{~nm}$, which could be ascribed to decomposition species. An NMR study of the conversion of the triflate complex to $\mathbf{1}$ showed clearly the resonances of a new complex consistent with the formulation presented and the THF and triflate in a trans- configuration. For the isolated triphenylphosphine analog 2, a single emission at $514 \mathrm{~nm}$ is observed.

For complex 1 prepared photochemically as soon as NO-donor was added to the solution, (10 $\mu \mathrm{M}$ to $250 \mu \mathrm{M}$ concentration range) the emission intensity at $585 \mathrm{~nm}$ decreased while the emission intensity at $435 \mathrm{~nm}$ increased and its maximum was shifted to $445 \mathrm{~nm}$ (Figures 3.1 and 3.2). However, it appears to be an anomaly for the $10 \mu \mathrm{M}$ and $20 \mu \mathrm{M}$ points, (Figure 3.2), since the trend is opposite for small additions of NOdonor/NaOH solution. This could be ascribed to the fact that the zero point does not have any $\mathrm{NaOH}$. Similar results were observed when the complex was prepared thermally (Figure 3). The emission at $585 \mathrm{~nm}$ appears to be quenching before the increase of the emission at $445 \mathrm{~nm}$ becomes prominent. This may be indicative of a two-step process, with a first step that may involve the partial dissociation of the THF ligand followed by radiation-less radical reactions, and a second step forming a species with a fluorescence emission at $445 \mathrm{~nm}$. As a control, experiments with (phen) $\operatorname{Re}(\mathrm{CO})_{3} \mathrm{Cl}$ and (phen) $\mathrm{Re}(\mathrm{CO})_{3} \mathrm{OSO}_{2} \mathrm{CF}_{3}$ under identical conditions showed no changes in the fluorescence spectra. 


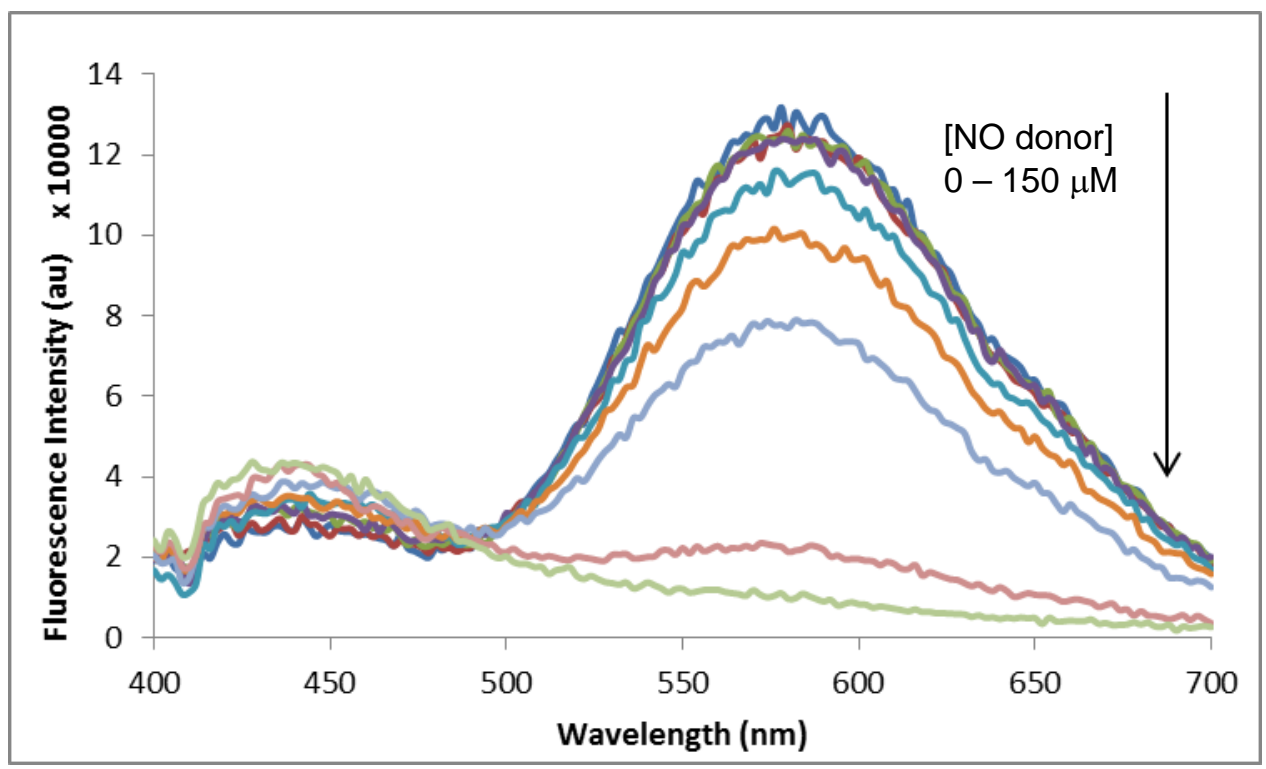

Figure 3.1 Fluorescence Response upon NO-donor addition $(0-150 \mu \mathrm{M})$ to $20 \mu \mathrm{M}$ of Re complex 2 (generated by photochemical substitution) in acetone/PBS aqueous buffer $25 / 75 \mathrm{v} / \mathrm{v},\left(\lambda_{\text {exc }}=360 \mathrm{~nm}\right)$

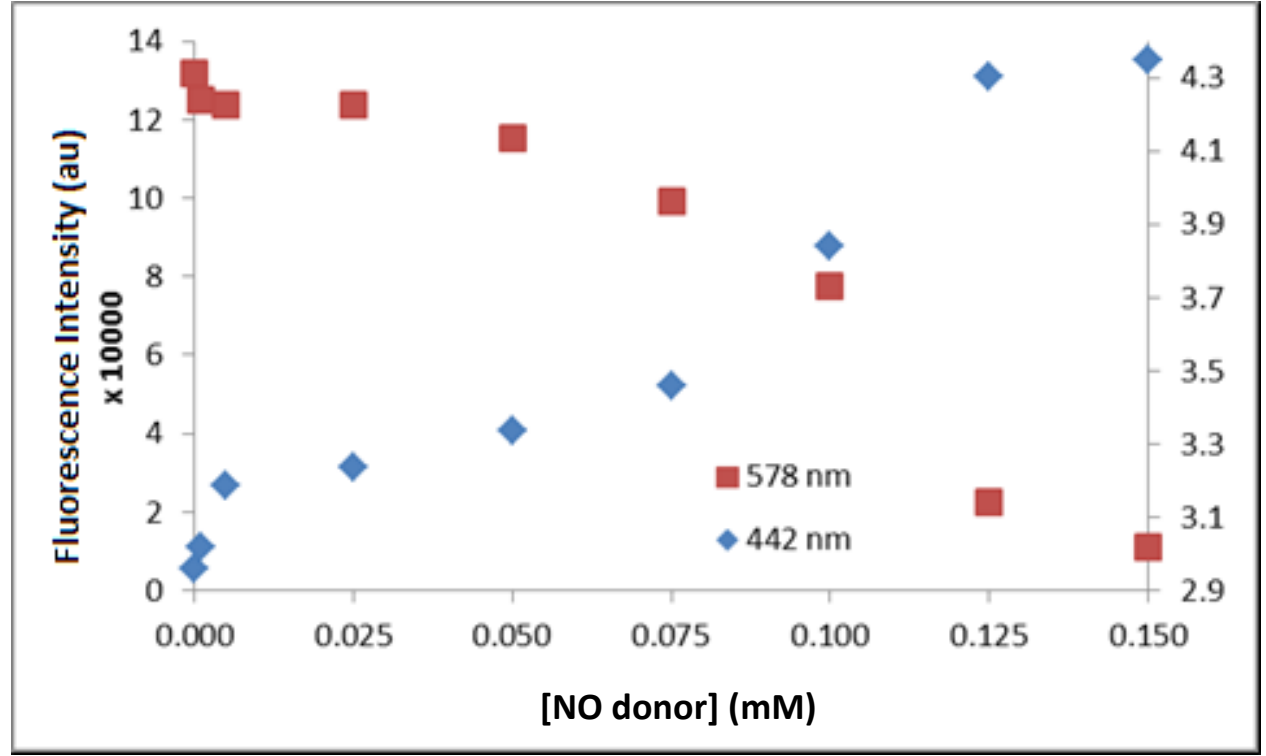

Figure 3.2 Observed Fluorescence intensities $\lambda_{\max }=450 \mathrm{~nm}$ and $\lambda_{\max }=582 \mathrm{~nm}$ upon NO-donor addition [2] $=40 \mu \mathrm{M}\left(\lambda_{\text {exc }}=360 \mathrm{~nm}\right)$. 


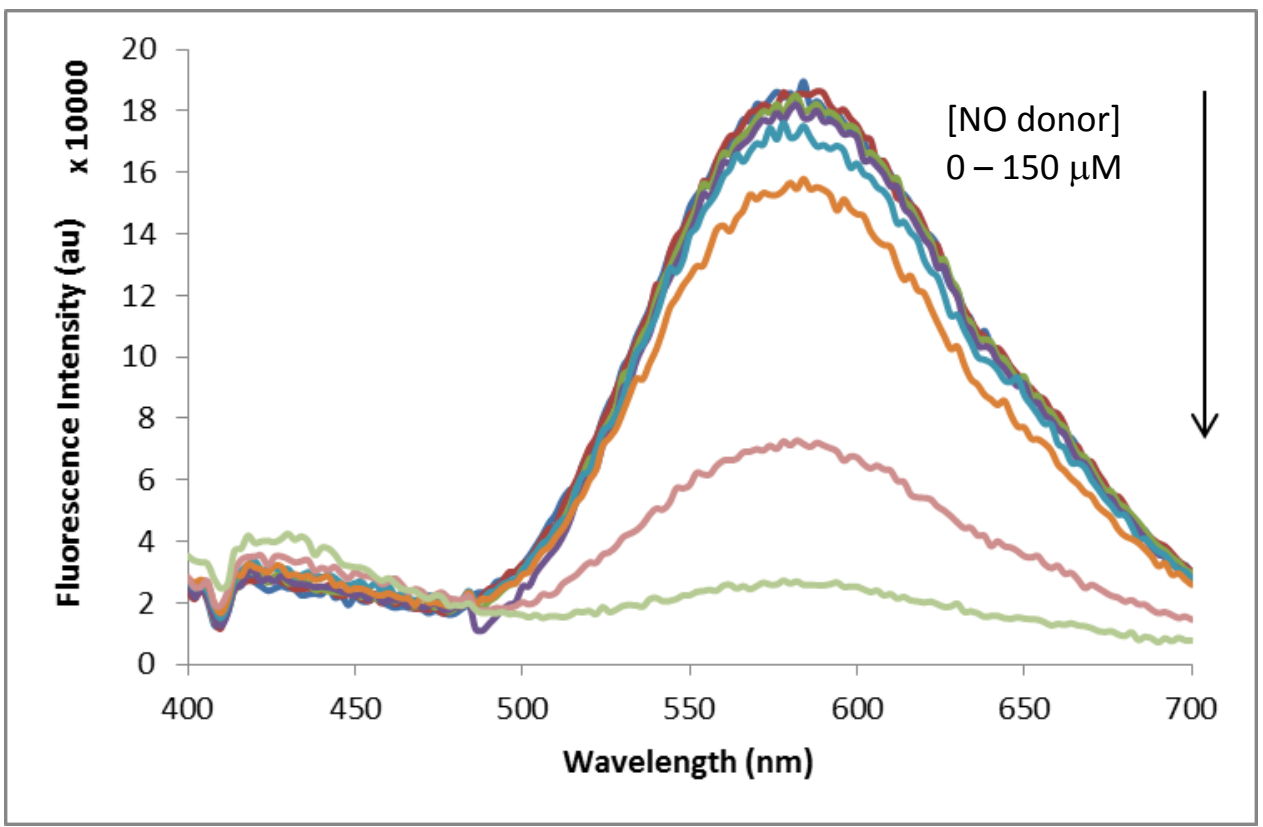

Figure 3.3 Fluorescence Response upon NO-donor addition $(0-150 \mu \mathrm{M})$ to $20 \mu \mathrm{M}$ of Re complex 2 (generated by thermal substitution) in acetone/PBS aqueous buffer $25 / 75 \mathrm{v} / \mathrm{v},\left(\lambda_{\text {exc }}=360 \mathrm{~nm}\right)$

Having similar results but not identical suggest that the degree of conversion may vary between the two methods but the identity of the final product is the same. A blank experiment was performed using only gradual increasing amounts of sodium hydroxide solution in absence of MAHMA NONOate; in the outcome there was no reaction at all, this confirm that it is the reaction with NO-donor the responsible of the change in fluorescence.

Fluorescence titration with gradual addition of NO-donor under anaerobic and aerobic conditions: For fluorescence titration measurements, a stock solution of (phen) $\mathrm{Re}(\mathrm{CO})_{3} \mathrm{OSO}_{2} \mathrm{CF}_{3}$ in THF with a mass concentration of $1 \mathrm{mg} / \mathrm{mL}$ was freshly prepared and irradiated at $300 \mathrm{~nm}$ in a 10 lamp photoreactor for 75 minutes, obtaining a solution of the complex. Then, $67 \mu \mathrm{L}$ of this stock solution was diluted in $683 \mu \mathrm{L}$ of acetone and $2250 \mu \mathrm{L}$ of phosphate buffer $0.002 \mathrm{M} \mathrm{pH} 7.2$ with sodium chloride $0.04 \mathrm{M}$ 
(resulting in a total volume of $3 \mathrm{~mL}$ and a final mass concentration of $25 \mu \mathrm{M}$ ) in a quartz glass cuvette. The fluorescence emission was monitored using $\lambda_{\text {exc }}$ of $360 \mathrm{~nm}$. The bandpass for excitation and emission was $2 \mathrm{~nm}$. A stock solution of MAHMA NONOate in $\mathrm{NaOH} 0.04 \mathrm{M}$ with a mass concentration of $3 \mathrm{mmol} / \mathrm{mL}$ was freshly prepared; this stock solution was dispensed stepwise in portions of $25 \mu \mathrm{L}$ from a $50 \mu \mathrm{L}$ syringe into the fluorescence cuvette. After each step, the solution was left react for 10 minutes (approximately, 3 MAHMA NONOate half-life) of at room temperature before measuring fluorescence using the same parameters as above.

In another cuvette, it was prepared other $3000 \mu \mathrm{L}$ of (phen)Re(THF)(CO) ${ }_{2} \mathrm{OSO}_{2} \mathrm{CF}_{3}$ in the same THF/acetone/buffer (see above) was bubbled with nitrogen for 30 minutes. The fluorescence titration was performed under the same conditions than above.

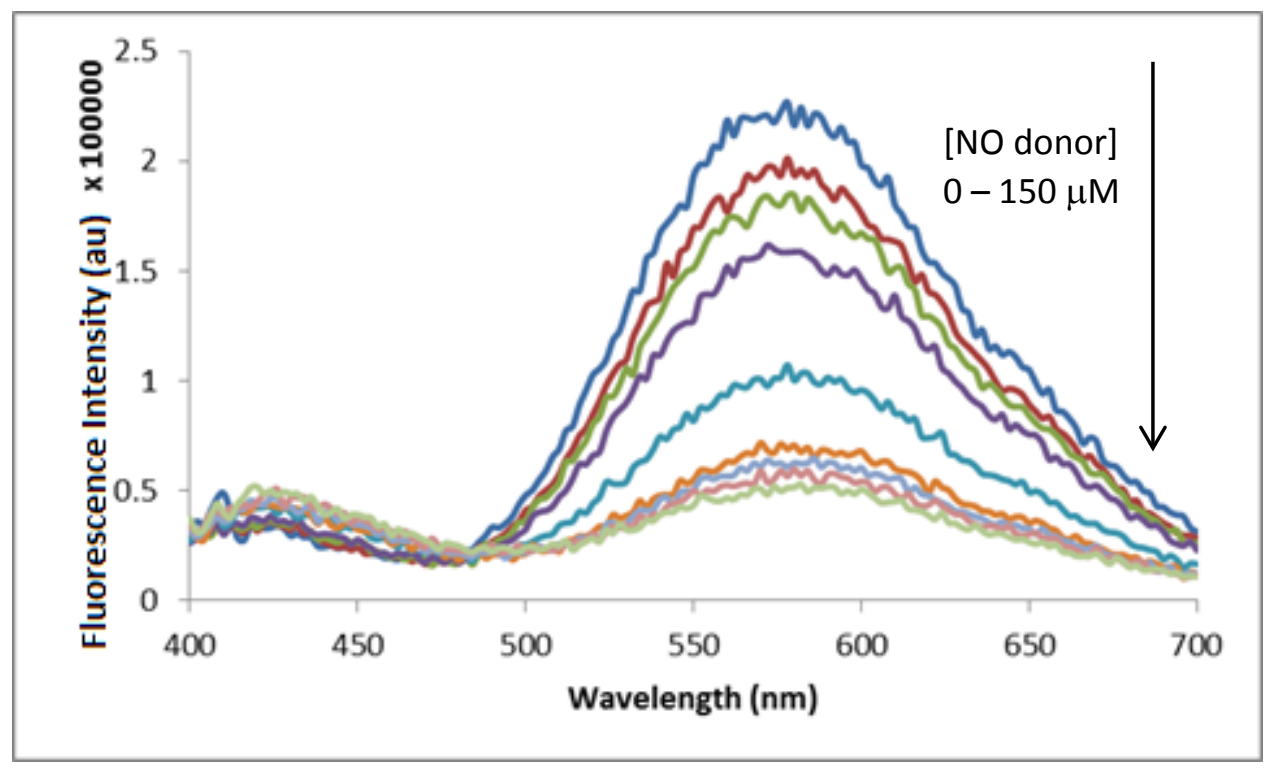

Figure 3.4 Fluorescence Spectra of $225 \mu \mathrm{M}$ with NO, acetone/buffer 25:75, $\lambda$ exc $=360 \mathrm{~nm}$, under nitrogen 


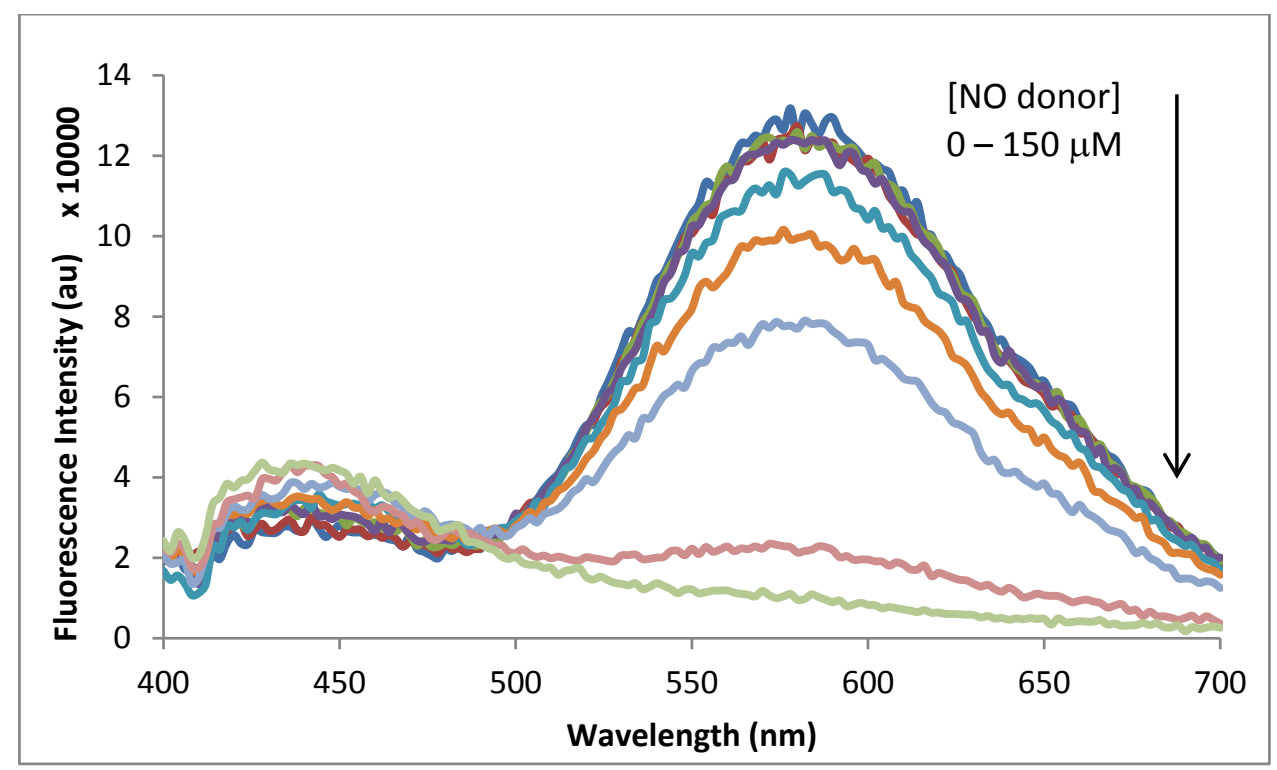

Figure 3.5 Fluorescence Spectra of $225 \mu \mathrm{M}$ with NO, acetone/buffer $25: 75, \lambda$ exc $=360 \mathrm{~nm}$

The results were as expected, quenching signal at $585 \mathrm{~nm}$ versus a slight increment in the signal around 430nm. After performing the titration under air or under nitrogen exhibiting similar behaviors, it can be concluded that it is no need to an oxidant environment in order the reaction to occur; like in the triazol formation based dyes. On the same tenor, it can be concluded that NO is the real substrate.

The intensity of the titration performed under air is $40 \%$ lower than under nitrogen, and the response towards NO addition is lower in the same extend, that can be explained by oxidation of the real substrate $\mathrm{NO}$ to $\mathrm{NO}_{2}$, and this second one cannot be used as a substrate for this Re complex.

One noticeable result is that in both cases the increment in the $430 \mathrm{~nm}$ signal is the same in both cases. It can be concluded that this signal depends only on the product after addition of NO; and no on the side reaction of the starting material. 


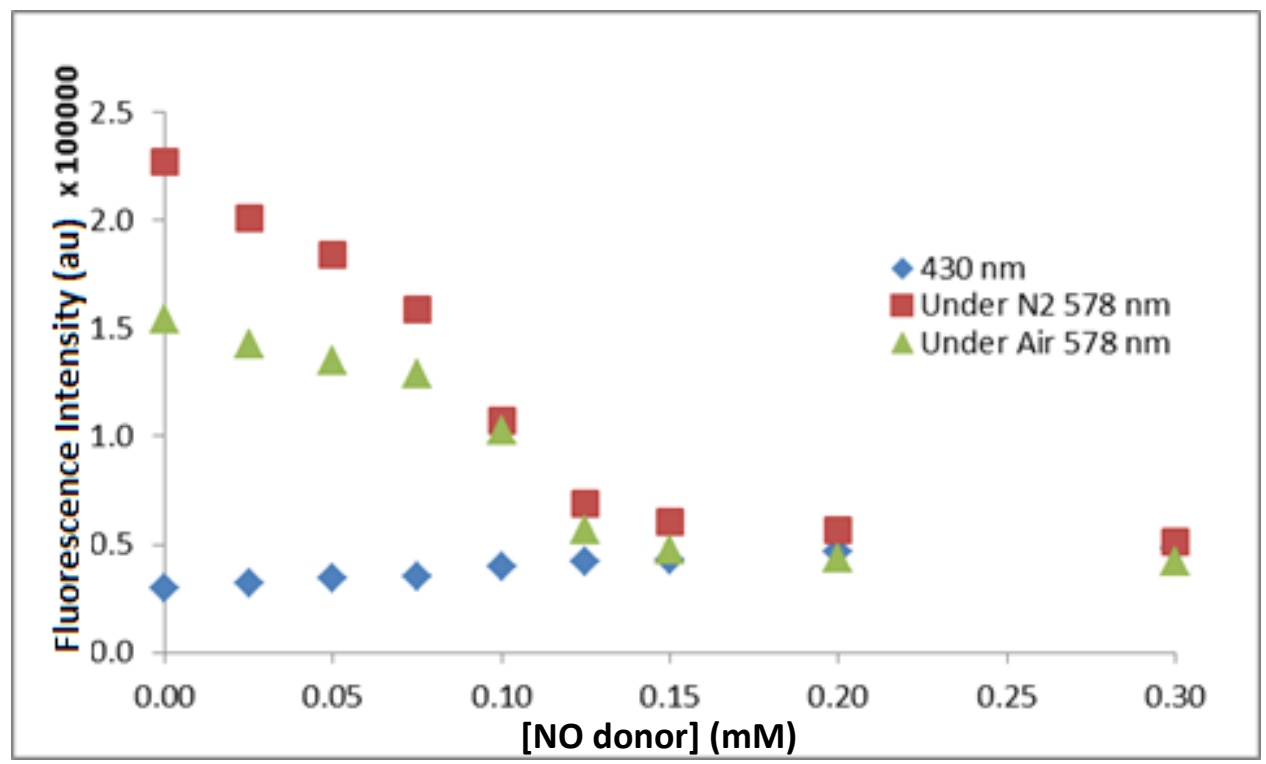

Figure 3.6 Fluorescence Spectra of (phen)Re(THF)(CO) ${ }_{2} \mathrm{OSO}_{2} \mathrm{CF}_{3} / \mathrm{THF} 30 \mu \mathrm{M}$ with $\mathrm{NO}$, acetone/buffer $25: 75, \lambda_{\text {exc }}=360 \mathrm{~nm}$, comparison nitrogen vs air

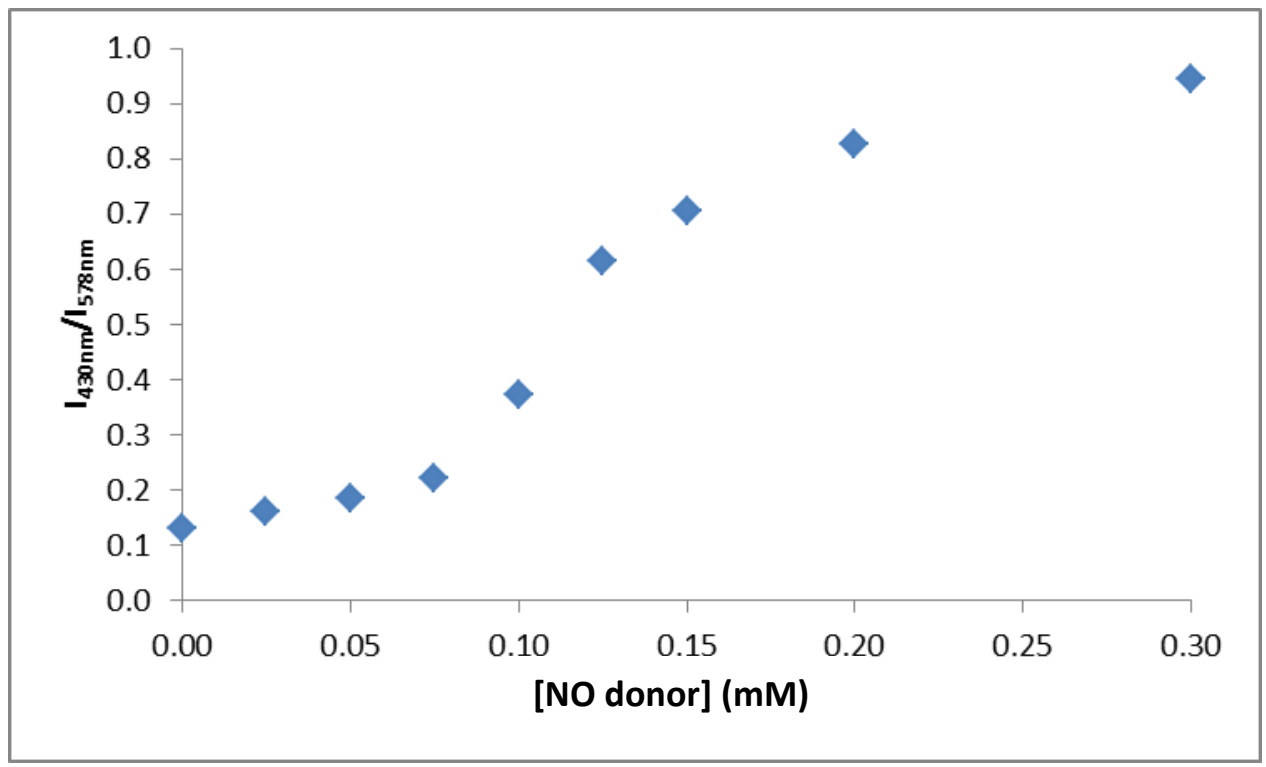

Figure 3.7 Fluorescence intensity ratio of $430 \mathrm{~nm} / 578 \mathrm{~nm}$ for $2(25 \mu \mathrm{M})$ with NOdonor addition (0 - $150 \mu \mathrm{M})$ 
There was a linear response at the lower range; until 3 times (phen) $\mathrm{Re}(\mathrm{THF})(\mathrm{CO})_{2} \mathrm{OSO}_{2} \mathrm{CF}_{3}$ complex concentration was reached; saturation was not reached.

\subsubsection{FT-IR spectroscopy:}

The FT-IR spectra were collected before (Fig. 3.8) and after the addition of NOdonor to $\mathbf{1}$. After adding NO, the solutions were treated by an acid/base wash $(1 \mathrm{M} \mathrm{HCl}$ / $1 \mathrm{M} \mathrm{NaHCO}_{3}$ in order to remove basic impurities, such as MAHMA-NONOate reaction products and uncoordinated phen and analogs, and the spectrum was collected (Fig. 3.9). In the spectrum of the product after NO addition bands were observed at $1720 \mathrm{~cm}^{-1}$ and $1631 \mathrm{~cm}^{-1}$, which are not present in the starting material and can be assigned to metalnitrosyl complexes. These typically show stretching bands in the $1600 \mathrm{~cm}^{-1}-1750 \mathrm{~cm}^{-1}$ range. ${ }^{18 c, 18 \mathrm{~d}}$ Nitroso compounds (organic NO) typically appear below $1600 \mathrm{~cm}^{-1}$. Even though NO stretching bands are typically strong they appear rather weak in our spectra, thus indicating that the Nitrosyl complex may not be the major product of the reaction of NO with the Re complex, nevertheless the presence of these bands, which do not appear when there is not reaction with NO, are indicative of a Re-NO complex. 


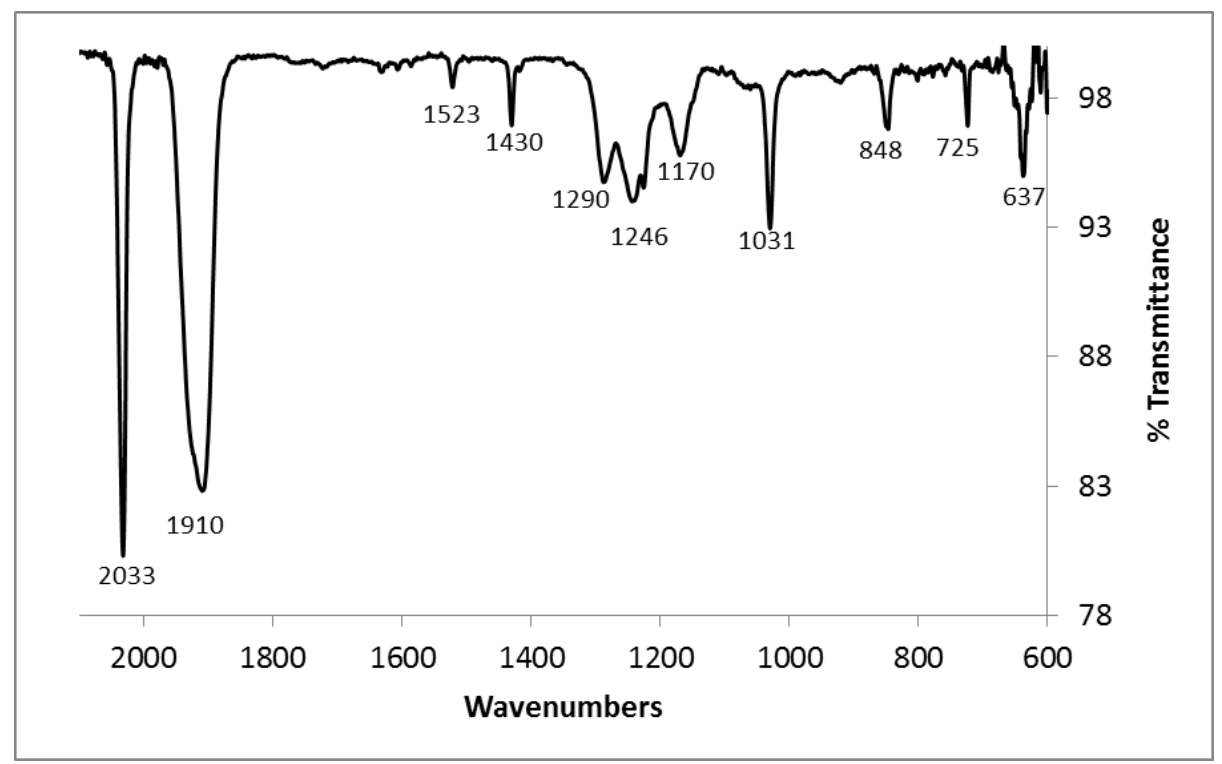

Figure 3.8 FT-IR spectrum of 2

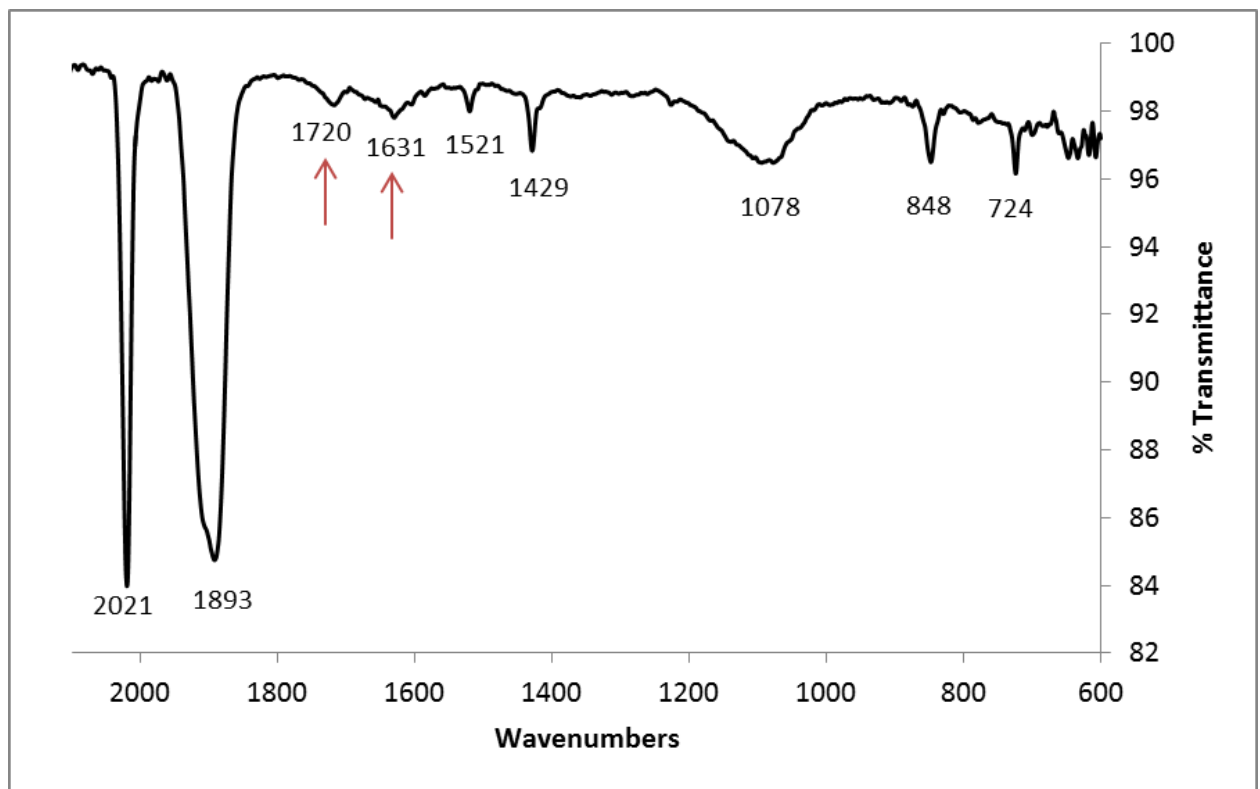

Figure 3.9 FT-IR spectrum of 2 after reaction with NO-donor and wash with $1 \mathrm{M}$ $\mathrm{HCl} / 1 \mathrm{M} \mathrm{NaHCO}$. 


\subsection{4. ${ }^{1}$ H-NMR and ${ }^{19}$ F-NMR Study of Re complex reactivity:}

\section{Formation of 1 from (phen) $\operatorname{Re}(\mathrm{CO})_{3}\left(\mathrm{OSO}_{2} \underline{\mathrm{CF}}_{3}\right)$ in $\mathrm{d}_{8}$-THF:}

Photosubstitution of (phen) $\mathrm{Re}(\mathrm{CO})_{3} \mathrm{OSO}_{2} \mathrm{CF}_{3}$ in $\mathrm{d}_{8}$-THF allowed monitoring of conversion into complex 1 by NMR spectroscopy (Fig. 2.10). Two different species (A and B) can be identified in the spectrum with integration of ratios of $80 / 20$ after $30 \mathrm{~min}$, while a third species (C) appears after 90 min with an integration ratio of 7 to 62(for A) to 32 (For B). These three species are presumed to be the starting material (A), and the trans-THF/triflate cis-CO complex (B), and the cis-THF/triflate cis-CO complex (C). The assignments are based on the higher observed symmetry of the spectral pattern for B.

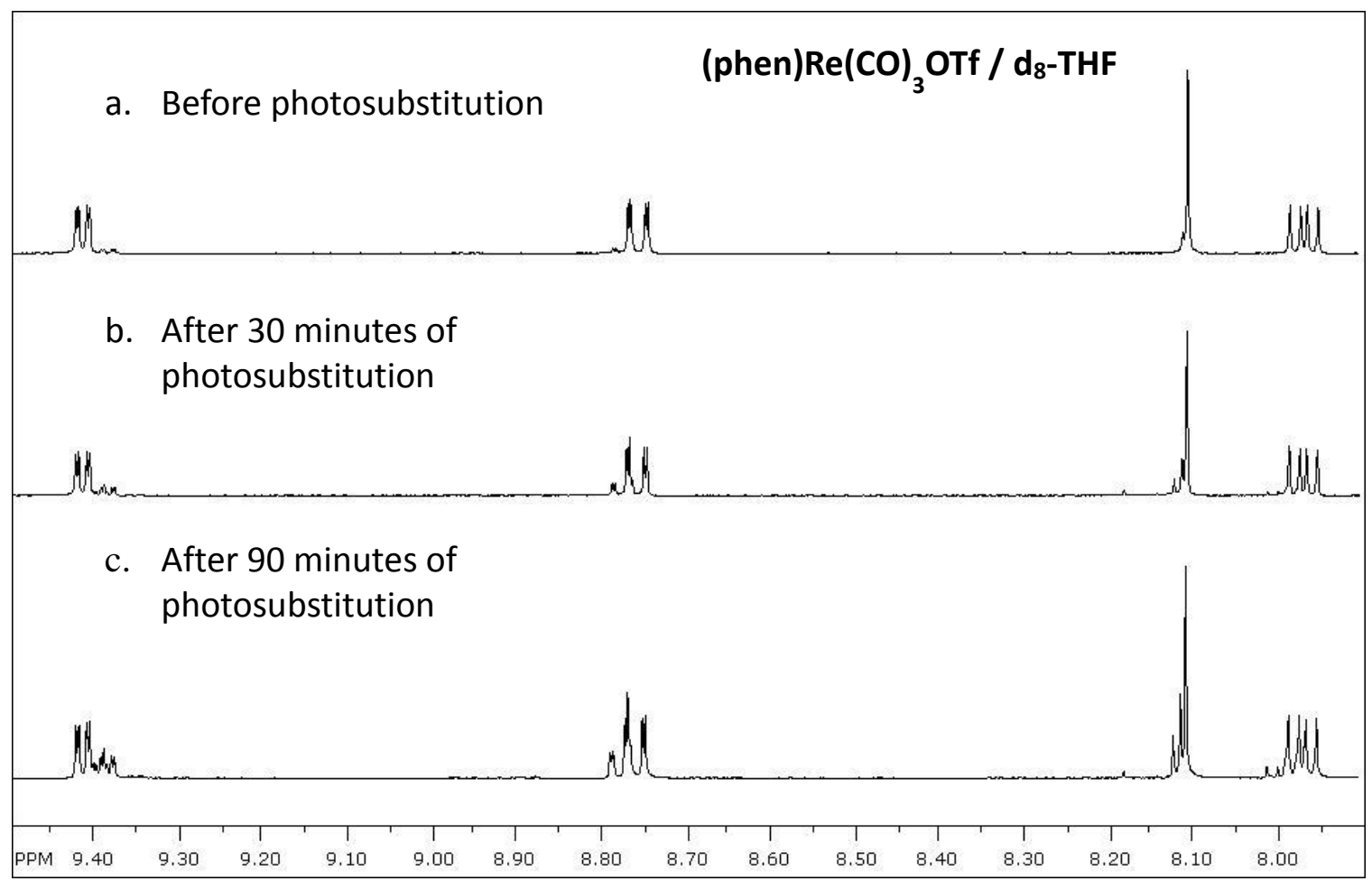

Figure 3.10 Aromatic region of ${ }^{1} \mathrm{H}-\mathrm{NMR}$ spectra (d8-THF) for $\mathbf{2}$ (top), $\mathbf{2}$ after 30 min of UV irradiation at $300 \mathrm{~nm}$ (middle), and after 90 min of UV irradiation (bottom).

Even before the reaction mixture was irradiated there was a small conversion to the trans-THF-triflate complex (Fig. 3.10a). The isolated and spectroscopically 
characterized phosphine analog $\mathbf{1}$, (Chapter 2), which is in the trans $\mathrm{PPh}_{3}$-triflate configuration was found to have analogous spectroscopic symmetric splitting pattern with the major product of the reaction in $\mathrm{d}_{8}$-THF assigned as trans- (Species B).

NMR study of reactivity of rhenium complexes with NO: $\quad$ The ${ }^{1} \mathrm{H}-\mathrm{NMR}$ spectra of (phen) $\operatorname{Re}(\mathrm{CO}){ }_{3} \mathrm{Cl}$ and phenanthroline do not show spectral changes under irradiation conditions with THF, and futhermore no spectroscopic changes are observed even after the addition of NO-donor to the irradiated solutions. The triflate complex however showed some spectral changes upon reaction with 20-fold excess of NO donor (or 40-fold excess of NO as each MAHMA-NONOate gives 2 NO molecules which can be compared with the NMR spectra of complexes $\mathbf{1}$ and $\mathbf{2}$ under the same conditions(Figure 3.11a).

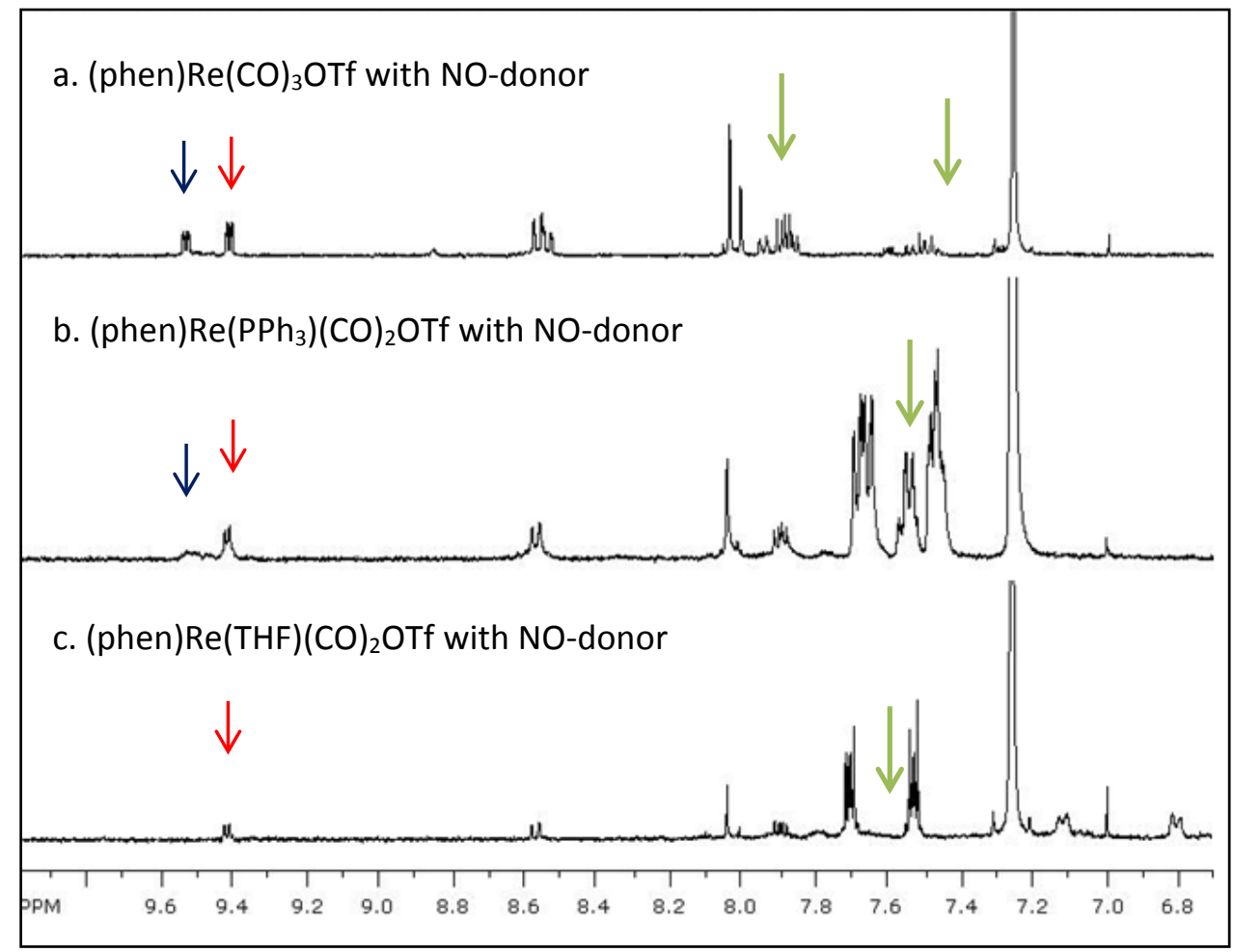

Figure 3.11 Aromatic region of ${ }^{1} \mathrm{H}-\mathrm{NMR}$ spectra after reaction with NO-donor ( $\mathrm{d}_{8}$-THF) of (a) (phen) $\mathrm{Re}(\mathrm{CO})_{3} \mathrm{OSO}_{2} \mathrm{CF}_{3}$, (b): $\mathbf{1}$ (c): 2. 
The spectrum of (phen) $\mathrm{Re}(\mathrm{CO})_{3} \mathrm{OSO}_{2} \mathrm{CF}_{3}$ upon reaction with NO shows two sets of phenanthroline signals (4 signals), and the appearance of a new set of signals at $\delta 7.4$ and $7.8 \mathrm{ppm}$ (Figure 3.11a). For the phosphine complex the integration between the two sets of signals (products vs. starting material) shows higher ratio of products vs. starting material, with an additional set of signals overlapping with the triphenylphosphine signal (Fig. 3.11b). The integration ratios and chemical shift changes indicate triphenylphospine dissociation. Phenanthroline is also dissociated upon NO reaction and phenanthrolinecontaining byproducts are formed. For the THF complex 2, the signals from the starting material are completely consumed; signals at $\delta=7.4$ and $7.8 \mathrm{ppm}$ are the most intense and those signals do not exhibit phenanthroline splitting pattern. Integration of THF resonances in the 1.8 - 2.8 ppm region also indicate THF dissociation from Re upon reaction with NO.

Acid/Base wash studies: Phenanthrolines and substituted phenanthrolines, which are not bound to the rhenium center, are basic and can be removed (together with the amines formed as byproducts from reaction of MAHMA NONOate) by acid wash with $1.0 \mathrm{M}$ $\mathrm{HCl}$, (Fig. 3.12). ${ }^{23}$ After 2 reacts with NO-donor (5-fold excess) it displays a complicated spectrum, (Fig. 3.12a) caused by the presence of six different species, of which only two appear to contain Re. After treatment with $1 \mathrm{M} \mathrm{HCl}$, and then $1 \mathrm{M} \mathrm{NaHCO}$, the basic organic species are removed, and only the Re-containing species remain in the spectrum. 


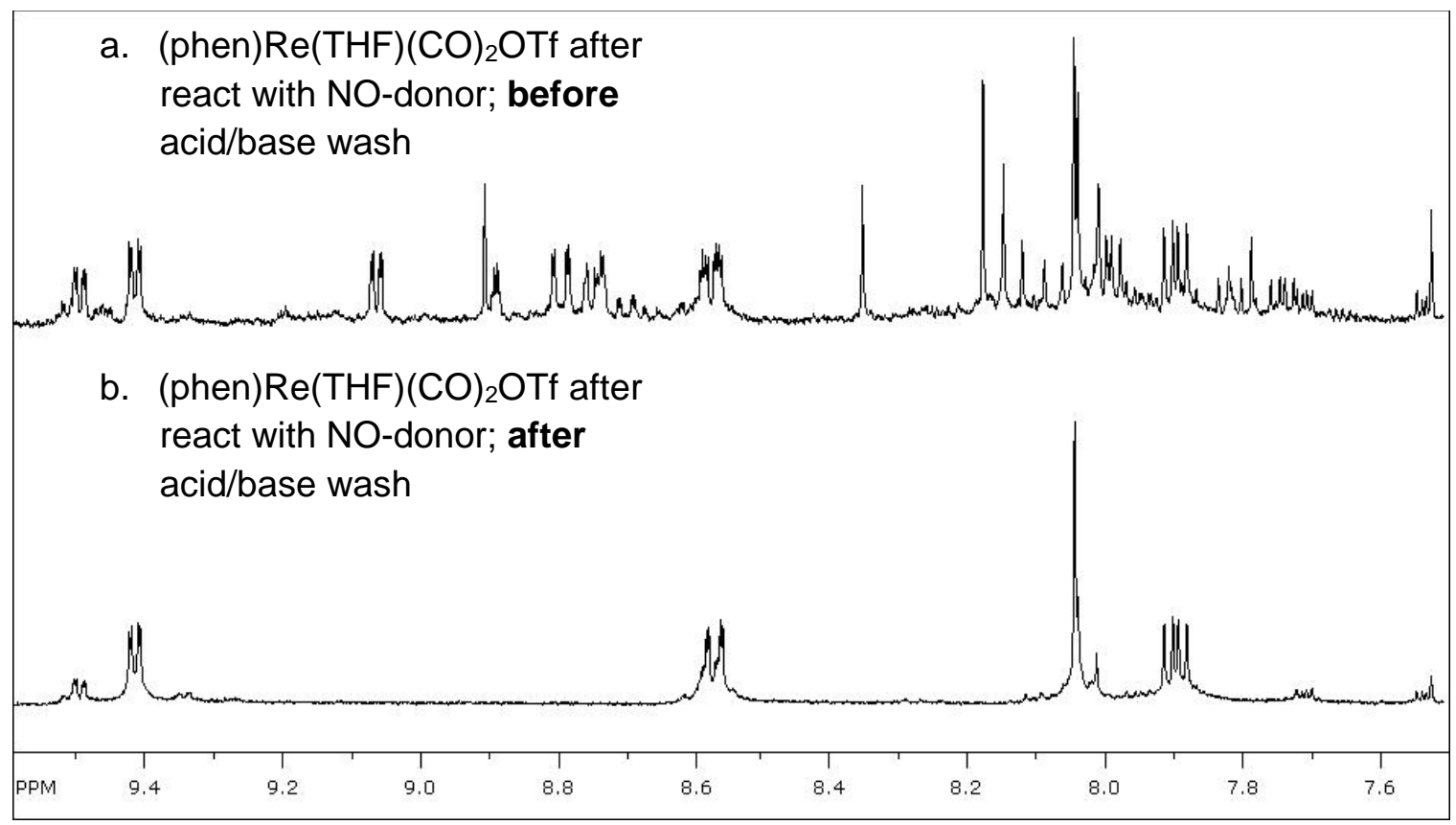

Figure 3.12 NMR spectra (aromatic region) for the reaction of $\mathbf{2}$ with NO-donor before (a) and after (b) wash with $1 \mathrm{M} \mathrm{HCl} / 1 \mathrm{M} \mathrm{NaHCO}$.

One of the species present is (phen) $\mathrm{Re}(\mathrm{CO})_{3} \mathrm{Cl}$, possibly formed after reaction of the coordinatively unsaturated $\mathrm{Re}(\mathrm{I})$ center resulting after dissociation of THF and $\mathrm{OSO}_{2} \mathrm{CF}_{3}{ }^{-}$ligands. The chemical shifts of the second species are completely different from complex 2 and are consistent with a species that has no longer contains a coordinated $\mathrm{CF}_{3} \mathrm{SO}_{3}{ }^{-}$ligand and in which the THF has been replaced by a $\pi$-acid such as NO. In summary, with the exception of (phen) $\mathrm{Re}(\mathrm{CO}){ }_{3} \mathrm{Cl}$, all the $\mathrm{NMR}$ spectra show a pattern of a) signals corresponding to non-coordinated to Re ortho-di-substituted phenanthroline which practically disappear after treatment with $1 \mathrm{M} \mathrm{HCl} / 1 \mathrm{M} \mathrm{NaHCO} 3$ b) signals corresponding to possibly a Re nitrosyl complex (or complexes) and c) signals corresponding to (phen) $\mathrm{Re}(\mathrm{CO})_{3} \mathrm{Cl}$ presumably formed from unreacted (phen) $\mathrm{Re}(\mathrm{CO})_{3} \mathrm{OSO}_{2} \mathrm{CF}_{3}$. The intensity patterns for those peaks indicate a decrease in reactivity moving from 2 , to $\mathbf{1}$, to (phen) $\mathrm{Re}(\mathrm{CO})_{3} \mathrm{OSO}_{2} \mathrm{CF}_{3}$. 
These experiments underscore the need for a labile L-type ligand, and suggest that triphenylphosphine dissociates in order for NO to react with the metal center. ${ }^{19} \mathrm{~F}$ NMR after NO-donor reaction showed no coordinated triflate signals at $-78.4 \mathrm{ppm}$, before or after acid wash. Thus, it can be concluded that $\mathrm{CF}_{3} \mathrm{SO}_{3}{ }^{-}$got dissociated after reaction with $\mathrm{NO}$, suggesting that $\mathrm{NO}$ displaces both triflate and $\mathrm{PPh}_{3}$ in order to coordinate to $\operatorname{Re}(\mathrm{I})$.

\subsubsection{Cell Studies \& Fluorescence Microscopy:}

Fluorescent dyes should be able to detect NO in biological systems, such as cells and endothelial tissues, in order to be practical for NO imaging and study of mechanistic aspects of its physiology.

Reactivity of 2 in RAOEC: Fluorescent dyes should be able to give a visible fluorescence response to different intracellular NO concentrations. Fig 3.13 shows that increasing the concentration of $\mathrm{NO}$ leads to increases in fluorescence intensity. Beyond $10 \mu \mathrm{M} \mathrm{NO}$, the RAOEC cells showed signs of shrinking, indicative of cell death. 


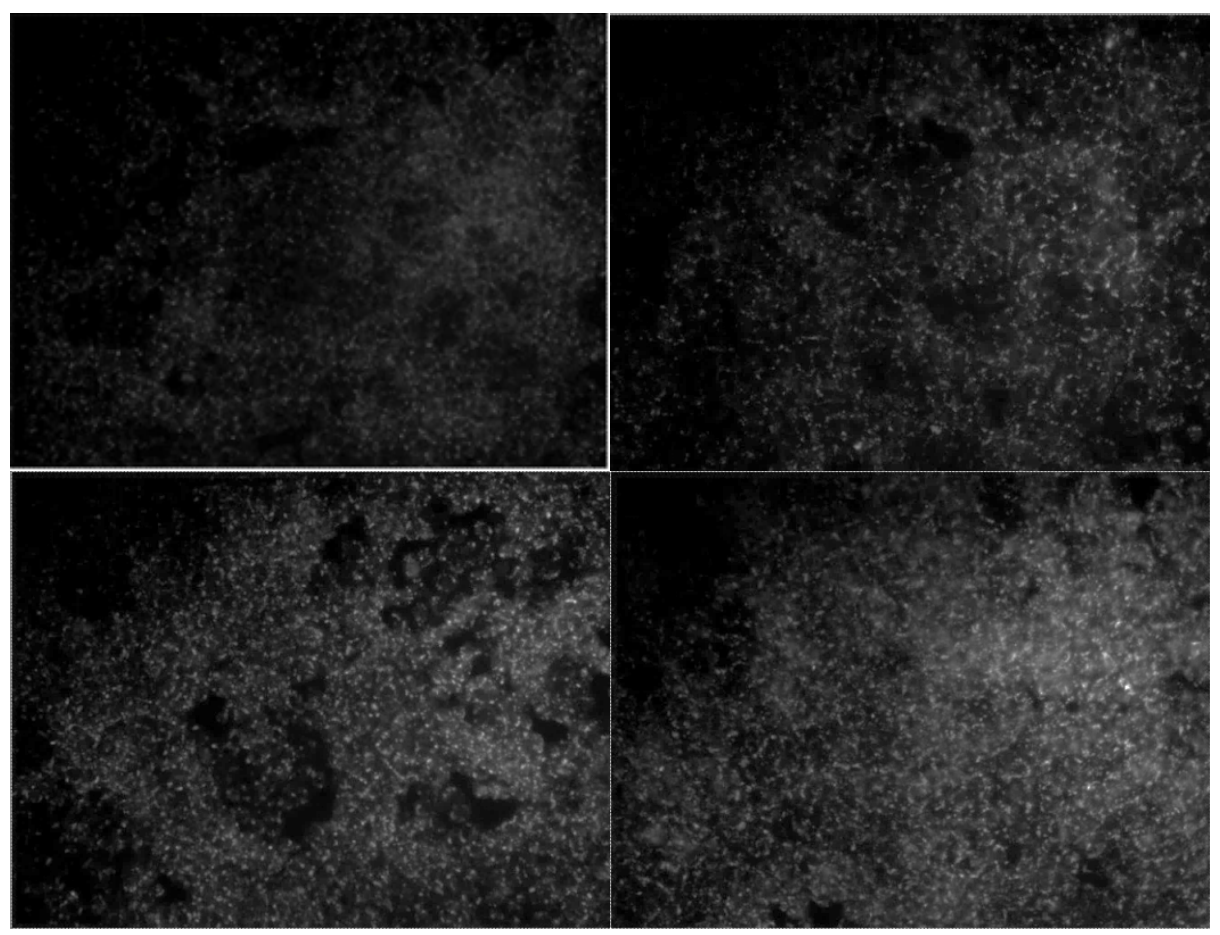

Figure 3.13 Picture sequence demonstrating fluorescence response in RAOEC at $\lambda_{\text {em }}=$ $450 \mathrm{~nm}$, Dye was generated by photochemical substitution (50 min). (a) with no NOdonor added (b) with $1 \mu \mathrm{M}$ NO-donor (c) with $5 \mu \mathrm{M}$ NO-donor and (d) with $10 \mu \mathrm{M}$ NOdonor. $\lambda_{\text {exc }}=350 \mathrm{~nm}$.

The reaction of rhenium complexes with NO-donor is complex and produces several species, some of them fluorescent. Both the NMR and fluorescence studies are consistent with an initial dissociation of the labile THF or $\mathrm{PPh}_{3}$ ligand, followed by reaction with NO either i) on the coordinated phenanthroline with subsequent dissociation from the metal center, as it has been observed before by $\mathrm{Hill}^{25}$, Mann ${ }^{26}$ and Halper $^{27}$ or ii) on the Re center forming a nitrosyl complex. The presence of $\mathrm{CFSO}_{3}{ }^{-}$in the Re coordination sphere is important for reaction with NO. Scheme 2 summarizes possible mechanistic pathways: 


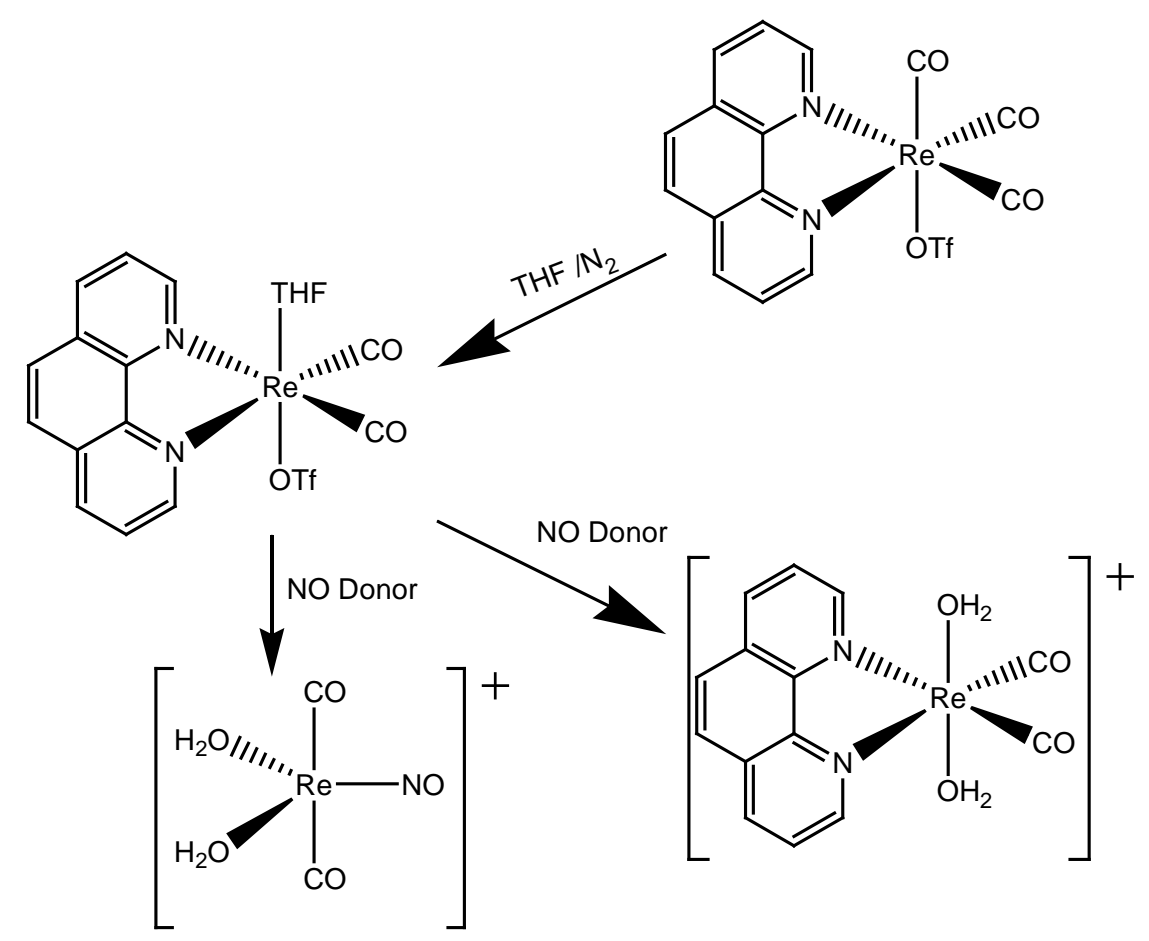

Scheme 3.2 Proposed mechanistic pathways for NO reactivity with (phen) $\mathrm{Re}(\mathrm{CO})_{2}(\mathrm{THF}) \mathrm{OSO}_{2} \mathrm{CF}_{3}$ (2)

\subsection{CONCLUSIONS}

The organometallic $\operatorname{Re}(\mathrm{I})$ complex was shown to react with $\mathrm{NO}$ at room temperature, producing fluorescence responses. The presence of labile ligands (THF, triflate, $\mathrm{PPh}_{3}$ ) is critical for reactivity with NO. Comparison studies show that rhenium complexes are prone to partial deactivation by side-reactions. These probes are able to permeate the cell membrane by diffusion and also exhibit fluorescence responses upon NO-donor addition inside the cell.

\subsection{REFERENCES}

1. Snyder, S. H.; Bredt, D. S. Sci Am. 1992, 266, 68-77.

2. (a) Tuteja, N.; Chandra, M.; Tuteja, R.; Misra, M. J.Biomed. Biotechnol., 2004, 4, 227-237; (b) Dredt, D. S. W.; Snyder, S. H. Ann. Rev. Biochem. 1994, 63, 175195; (c) Wink, D. A.; Vodovotz, Y.; Laval, J.; Laval, F.; Dewhirst, M. W.; Mitchell, J. B. "The multifaced roles of nitric oxide in cancer". Carcinogenesis, 
1998, 19, 711-721; (d) Calabrese, V.; Bates, T. E.; Stella, A. M. G. Neurochem. Res., 2000, 25, 1315-1341. (e) Marletta, M. A.; Hurshman, A. R.; Rusche, K. M. Curr. Opin. Chem. Biol. 1998, 2, 656-663. (f) Ricciardolo, F. L. M.; Sterk, P. J.; Gaston, B.; Folkerts, G. Physiol. Rev. 2004, 84, 731-765. (g) Packer, L. Methods in Enzymology, Nitric Oxide. Part B, Physiological and Pathological Processes; Academic Press: San Diego, CA, 1996; Vol. 269.

3. (a) Singh, S., Gupta, A.K. Cancer Chemother Pharmacol., 2011, 67, 1211-1224;

4. (a) Bates, J. N. Neuroprotocols, 1992, 1, 99-107. (b) Marletta, M. A. Trends in Biochemical Sciences, 1989, 14, 488-492. (c) Ischiropoulos, H.; Gow, A. Toxicology. 2005, 208, 299-303.

5. Tsoukias, N. M.; Popel, A. S. American Journal of Physiology 2002, 282, H2265-77. (b) Tsoukias N. M. Microcirculation 2008, 15, 8.

6. Kavdia M.; Tsoukias N. M.; Popel, A. S. American Journal of Physiology 2002, 282, H2245-53.

7. Tsoukias, N.M.; Popel, A. S. Microcirculation 2003, 10, 479-95.

8. (a) Tsoukias, N. M.; Kavdia, M.; Popel A. S. American Journal of Physiology 2004, 286, H1043-56. (b) Madrasi K.; Joshi, M.S.; Gadkari T.; Kavallieratos, K.; Tsoukias, N. M. Free Radic. Biol. Med., 2012, 53, 1968-1976.

9. Kashiwagi, S., Tsukada, K., Xu, L., Miyazaki, J., Kozin, S. V., Tyrrell, J.A., Sessa, W. C., Gerweck, L.E., Jain, R.K., Fukumura, D. Nature Medicine 2008, $14,255-257$.

10. (a) Loscalzo J. Arterioscler. Thromb. Vasc. Biol., 2001, 21, 1259-1260. (b) Moncada, S.; Higgs, A. N. Engl. J. Med., 1993, 329, 2002-20012.

11. Namin, S. M.; Nofallah, S.; Joshi, M. S.; Kavallieratos, K.; Tsoukias, N. M. Nitric Oxide, Biology and Chemistry, 2013, 28, 39-46.

12. Kojima, H., Nagano, T. Chem. Rev., 2002, 102, 1235-1269.

13. (a) Moncada, S.; Palmer, R. M. J.; Higgs, E. A. Pharmacol. Rev., 1991, 43, 109142; (b) Furchgot, R. F.; Vanhoutte, P. M. FASEB J,. 1989, 3, 2007; (c) Lancaster Jr., J. R., Nitric Oxide Biology and Pathology, ed. Ignarro, L.J., Academic Press, San Diego, 2000.

14. (a) Kojima, H.; Nakatsubo, N.; Kikuchi, K.; Kawahara, S.; Kirino, Y.; Nagoshi, H.; Hirata, Y.; Nagano, T. Anal. Chem., 1998, 70, 2446-2453. (b) Kojima, H.; Urano, Y.; Kikuchi, K.; Tsunehiko, H.; Hirata, Y. Nagano, T. Agnew. Chem. Int. Ed. Engl., 1999, 38, 3209-3212. 
15. Lim, M. H; Xu, D.; Lippard, S. J. Nature Chem Biol., 2006, 2, 375-380.

16. (a) Hu, X.; Wang, J.; Zhu, X.; Dong, D.; Zhang, X.; Wu, S.; Duan, C. Chem. Commun., 2011, 47, 11507-11509; (b) Victor, E., Kim, S., Lippard, S. Inorg.Chem. 2014, 53, 12809-1221. (c) Yuan, L.; Lin W.; Xie Y.; Chen B.; Song J. Chem. Commun., 2011, 47, 9372-9374.

17. (a) Martínez-Máñez, R. Inorg. Chem. Commun., 2000, 3, 45-48. (b) Dilworth, J. R. Dalton Trans., 2010, 39, 5219-5220. (c) Mingos, D. M. P. Chem. Soc. Rev. 1995, 24, 329. (d) Chi-Ming Che. Eur. J. Inorg. Chem. 2003, 255-62. Inorg. Chem., 2007, 46, 740-749.

18. (a) Armaola, N.; De Cola, L.; Balzani, V.; Sauvage, J. P.; Dietrich-Buchecker, C. O.; Kern, J. M. J. Chem. Soc., 1992, 88, 553-556. (b) Patra, A. K., Rose, M., Murphy, K. A., Olmstead, M. M., Mascharak, P. K. Inorg. Chem. 2004, 43, 44874495. (c) Pratihar, P.; Dasmahapatra, A. K.; Sinha, C. Polyhedron, 2007, 26, 1217-1221. (d) Machura, B. Coordination Chemistry Reviews, 2005, 249, 22772307.

19. Choi, A. W. T.; Poon, C. S.; Liu, H. W.; Cheng, H. K.; Lo, K. New J. Chem., 2013, 37, 1711-1719.

20. Fry, N. L.; Mascharak, P. K. Acc. Chem. Res., 2011, 44, 289-298.

21. Wrighton, M., Morse, D. L. J. Am. Chem. Soc., 1974, 96, 998-1003.

22. Sullivan, B.P., Meyer, T.J. J. Chem. Soc., Chem. Commun., 1984, 1244-1245.

23. $20.0 \mathrm{mg}$ of (phen) $\mathrm{Re}(\mathrm{CO})_{3} \mathrm{OSO}_{2} \mathrm{CF}_{3}(33.3 \mu \mathrm{mol})$ and $10 \mathrm{~mL}$ of anhydrous $\mathrm{THF}$ (final concentration of $3.33 \mathrm{mM}$ ) were placed in a $50 \mathrm{~mL}$ round bottom flask, under $\mathrm{N}_{2}$ flow. The solution was refluxed for $48 \mathrm{~h}$. The color changed during reflux from yellow to amber. The volatiles were evaporated to dryness and 1.0 $\mathrm{mL}$ of dichloromethane was added. The product was precipitated at room temperature by adding $4.0 \mathrm{~mL}$ of diethyl ether. The bright yellow powder product was filtered and was dried in vacuo at $40^{\circ} \mathrm{C}$.

24. (a) Alberto, R. Eur. J. Inorg. Chem. 2009, 1, 21-31. (b) Gottschaldt, M.; Koth, D.; Müller, D.; Klette, I.; Rau, S.; Görls,H.; Schäfer, B.; Baum, R. P.; Yano, S. Chem. Eur. J. 2007, 13, 10273-10280. (c) Zhang, X.; Chen, X. Appl. Radiat. Isot. 2007, 65, 70-78.

25. Hill, R. H.; Puddephatt, R. J. J. Am. Chem. Soc., 1985, 107, 1218-25.

26. Florence, T. M.; Stauber, J. L.; Mann, K. J. J. Inor. Bioch., 1985, 24, 243-54.

27. DeArmond, K.; Halper, W. J. Phys. Chem. 1971, 75, 3230-4. 


\section{STUDY OF THE FLUORESCENT ORGANOMETALLIC COMPLEX (phen)Re( $\left(\mathrm{CH}_{3} \mathrm{CN}\right)(\mathrm{CO})_{2} \mathrm{OSO}_{2} \mathrm{CF}_{3}$ AS A NO SENSOR.}

\subsection{ABSTRACT}

The complex (phen) $\mathrm{Re}\left(\mathrm{CH}_{3} \mathrm{CN}\right)(\mathrm{CO})_{2} \mathrm{OSO}_{2} \mathrm{CF}_{3}$ (3), which can be obtained thermally from (phen) $\mathrm{Re}(\mathrm{CO})_{3} \mathrm{OSO}_{2} \mathrm{CF}_{3}$ shows fluorescence quenching response upon NO addition, which was added in the form of NO-donor Z-1-N-methyl-N-[6-(Nmethylammoniohexyl)amino]diazen-1-ium-1,2-diolate (MAHMA-NONOate). Addition of 10-150 $\mu \mathrm{M}$ of NO-donor into a $25 \mu \mathrm{M}$ solution of (phen) $\mathrm{Re}\left(\mathrm{CH}_{3} \mathrm{CN}\right)(\mathrm{CO})_{2} \mathrm{OSO}_{2} \mathrm{CF}_{3}$ in 25\% v/v acetone/phosphate saline (PBS) buffer gave fluorescence quenching at 532 nm, upon excitation at $360 \mathrm{~nm}$. The ${ }^{1} \mathrm{H}-\mathrm{NMR}$ and ${ }^{19} \mathrm{~F}-\mathrm{NMR}$ spectra after reaction with NO showed characteristic resonances for non-coordinated triflate and $\mathrm{CH}_{3} \mathrm{CN}$, suggesting that the organometallic product formed upon NO releases both these ligands upon reaction with NO. The present study expands our newly demonstrated approach for the design of fluorescent NO sensors based on NO coordination to Re; ${ }^{1} \mathbf{3}$ is an isolable complex, which is stable at room temperature and therefore can be used as an NO sensing organometallic dye, as prepared, without further treatment.

\subsection{INTRODUCTION}

Acetonitrile transition metal complexes have been used as intermediaries in inorganic and organometallic synthesis. ${ }^{2} \quad$ These complexes are stable at room temperature and react only under photochemical conditions or at high temperatures. ${ }^{3}$ Rhenium acetonitrile complexes are typically synthesized by reflux in acetonitrile ${ }^{4}$ or using trimethylamine $\mathrm{N}$-oxide dehydrate. ${ }^{5}$ 
Rhenium tricarbonyl complexes have interesting photophysical properties, and they are used as luminophores. ${ }^{6}$ Rhenium imaging probes have shown reliable responses. Changing fluorophores selectively may target different subcellular structures. Rhenium(I) reacts with specific biochemical agents localized in the cell, such as sulfurcontaining aminoacids in the mitochondria. ${ }^{7}$

In prior work (Chapters 2 and 3), it was shown that (phen) $\mathrm{Re}(\mathrm{THF})(\mathrm{CO})_{2} \mathrm{OSO}_{2} \mathrm{CF}_{3}$, and (phen) $\mathrm{Re}\left(\mathrm{PPh}_{3}\right)(\mathrm{CO})_{2} \mathrm{OSO}_{2} \mathrm{CF}_{3}$ prepared in situ thermally or photochemically from (phen) $\mathrm{Re}(\mathrm{CO})_{3} \mathrm{OSO}_{2} \mathrm{CF}_{3}$ have the ability to react with NO under physiological conditions, producing a fluorescence change. The new acetonitrile analog 3 introduced in this Chapter shows analogous fluorescence responses, that can be ascribed directly to NO complexation by Re, as indicated by NMR and IR spectroscopic studies. 3 also has the additional advantages that it can be prepared in an isolable form, and is stable at room temperature.

\subsection{EXPERIMENTAL SECTION}

\subsubsection{Materials and Methods}

The complexes (phen) $\mathrm{Re}(\mathrm{CO})_{3} \mathrm{Cl}$ and (phen) $\mathrm{Re}(\mathrm{CO})_{3} \mathrm{OSO}_{2} \mathrm{CF}_{3}$ were prepared as previously reported and were found spectroscopically identical to the reported compounds. ${ }^{8,9}$ All other materials (purchased from Aldrich Chemical Co., ACROS organics, Cayman Chemicals, or Alfa Aesar) were standard reagent grade and were used without further purification, unless otherwise noted. PBS buffer (0.002 $\mathrm{M}$ in total phosphate, $\mathrm{pH}=7.2,[\mathrm{NaCl}]=0.04 \mathrm{M}$ ) was prepared from $\mathrm{K}_{2} \mathrm{HPO}_{4}, \mathrm{KH}_{2} \mathrm{PO}_{4}$, and $\mathrm{NaCl}$ (all from Fisher Scientific). Water used for preparation of the buffers and experiments with Nitric oxide was filtered water $\left(18 \mathrm{M} \Omega \cdot \mathrm{cm}^{-1}\right)$ produced from a Millipore ${ }^{\circledR}$ nanopure 
diamond lab water system (Barnstead Thermolyne Corporation, Dubuque, IA). Deuterated solvents were purchased from Cambridge Isotope Laboratories Ltd. ${ }^{1} \mathrm{H}$ and ${ }^{13} \mathrm{C}$ NMR spectra were recorded on a 400 Bruker NMR spectrometer and were referenced, using the residual solvent resonances. All chemical shifts, $\delta$, are reported in ppm. FT-IR spectra were recorded with a Perkin Elmer Spectrum 100 FT-IR Spectrometer. Frequency-domain fluorescence lifetime measurements were performed using a ChronosFD spectrofluorometer (ISS, Champaign, IL). Samples were excited with a $370 \mathrm{~nm}$ modulated diode, and emission was collected using $400 \mathrm{~nm}$ long-pass filters (Andover, Salem, NH).

\subsubsection{Synthesis of (phen) $\mathrm{Re}\left(\mathrm{CH}_{3} \mathrm{CN}\right)(\mathrm{CO})_{2} \mathrm{OSO}_{2} \mathrm{CF}_{3}$ :}

$20.0 \mathrm{mg}$ of (phen) $\mathrm{Re}(\mathrm{CO})_{3} \mathrm{OSO}_{2} \mathrm{CF}_{3}(33.3 \mu \mathrm{mol})$ and $20 \mathrm{~mL}$ of acetonitrile (final concentration: $16.5 \mathrm{mM}$ ) were placed in a $50 \mathrm{~mL}$ round bottom flask under $\mathrm{N}_{2}$ flow. The solution was refluxed for 48 hours. The color changed during reflux from yellow to amber. The volatiles were reduced to $1.0 \mathrm{~mL}$. The product was precipitated at room temperature by adding $4.0 \mathrm{~mL}$ of diethyl ether. The bright yellow powder product was filtered and was dried in vacuo at $40{ }^{\circ} \mathrm{C} .18 .4 \mathrm{mg},(84 \%) .{ }^{1} \mathrm{H}-\mathrm{NMR}\left(\mathrm{CDCl}_{3}\right): 2.18(3 \mathrm{H}$, s), $8.04\left(\mathrm{~d}, 2 \mathrm{H},{ }^{3} \mathrm{~J}_{\mathrm{H}-\mathrm{H}}=8.3 \mathrm{~Hz},{ }^{3} \mathrm{~J}_{\mathrm{H}-\mathrm{H}}=5.1 \mathrm{~Hz}\right), 8.20(\mathrm{~s}, 2 \mathrm{H}), 8.79\left(\mathrm{~d}, 2 \mathrm{H},{ }^{3} \mathrm{~J}_{\mathrm{H}-\mathrm{H}}=8.3\right.$ $\left.\mathrm{Hz},{ }^{3} \mathrm{~J}_{\mathrm{H}-\mathrm{H}}=1.3 \mathrm{~Hz}\right), 9.37\left(\mathrm{~d}, 2 \mathrm{H},{ }^{3} \mathrm{~J}_{\mathrm{H}-\mathrm{H}}=5.1 \mathrm{~Hz},{ }^{3} \mathrm{~J}_{\mathrm{H}-\mathrm{H}}=1.2 \mathrm{~Hz}\right) .{ }^{13} \mathrm{C}-\left\{{ }^{1} \mathrm{H}\right\}-\mathrm{NMR}$ ((CD $\left.\left.)_{2}\right)_{2} \mathrm{CO}\right): 3.17$ (CH3 $\left.{ }_{3} \mathrm{CN}, \mathrm{s}, 1 \mathrm{C}\right), 127.93(\mathrm{CH}, \mathrm{s}, 2 \mathrm{C}), 129.11(\mathrm{CH}, \mathrm{s}, 2 \mathrm{C}), 132.27(\mathrm{CH}$, s, 2C), 147.84 (CN, s, 1C), $155.74(\mathrm{CH}, \mathrm{s}, 2 \mathrm{C}) .{ }^{19} \mathrm{~F}-\left\{{ }^{1} \mathrm{H}\right\}-\mathrm{NMR}\left(\mathrm{CDCl}_{3}\right)$ : -78.2 ppm. FT-IR $\left(\mathrm{cm}^{-1}\right)$ : 2295 ( $\left.v_{\mathrm{cN}}, \mathrm{w}\right), 2033\left(v_{\mathrm{CO}}, \mathrm{s}\right), 1927\left(v_{\mathrm{CO}}, \mathrm{s}\right), 1904$ ( $\left.v_{\mathrm{co}}, \mathrm{s}\right)$, 1631, 1521, 1431, 1417, 1255, 1222, 1156, 1030, 877, 851, 724, 637. UV-Vis (in ethanol): $\lambda_{\max }=360$ $\mathrm{nm}$ - br $\left(\varepsilon=1840 \mathrm{~mol}^{-1} \mathrm{~cm}^{-1}\right)$, Fluorescence emission in acetone $\left(\lambda_{\text {exc }}=360 \mathrm{~nm}\right): 532 \mathrm{~nm}$ 
$\Phi_{1}=0.006$ (at $370 \mathrm{~nm}$, in 1:3 acetone/PBS buffer, using quinine sulfate in sulfuric acid $0.1 \mathrm{M}$ as reference $\left.\Phi_{\mathrm{R}}=0.546\right)$. Lifetime: $\tau_{1}=15.1 \pm 0.4 \mathrm{~ns}, \mathrm{f}_{1}=0.257 \pm 0.004, \alpha_{1}=$ $0.017 ; \tau_{2}=141 \pm 3 \mathrm{~ns}, \mathrm{f}_{2}=0.424 \pm 0.003, \alpha_{2}=0.00301$. There are two major processes occurring. The adding contributions only add up 70\%. There are minor reactions not fully described.

\subsubsection{Fluorescence experiments with NO-donor}

In a typical experiment, a $100 \mu \mathrm{M}$ solution of (phen) $\mathrm{Re}\left(\mathrm{CH}_{3} \mathrm{CN}\right)(\mathrm{CO})_{2} \mathrm{OSO}_{2} \mathrm{CF}_{3}$ complex was prepared from $1.67 \mathrm{mM}$ stock solution of (phen) $\mathrm{Re}\left(\mathrm{CH}_{3} \mathrm{CN}\right)(\mathrm{CO})_{2} \mathrm{OSO}_{2} \mathrm{CF}_{3}$ in $\mathrm{THF}$ and acetone was added up to $1.000 \mathrm{~mL}$ of total solution of Re complex. $2.75 \mathrm{~mL}$ to $3.00 \mathrm{~mL}$ of aqueous PBS buffer $(\mathrm{pH}=7.2)$ was added. Then 0 to $250 \mu \mathrm{L}$ of $4 \mathrm{mM}$ stock solution of MAHMA-NONOate NO-donor, in $0.04 \mathrm{M} \mathrm{NaOH}$, were added in order to obtain $75 \% \mathrm{v} / \mathrm{v}$ aqueous/organic solution and a final NO-donor concentration from 0 to $200 \mu \mathrm{M}$ range, while the concentration of $\mathrm{Re}$ complex remained constant at $25 \mu \mathrm{M}$. No more than 10 minutes elapsed from preparation of the organic phase to the addition of the NO-donor. The solution was mixed thoroughly in a vortex and sonicated for 90 minutes at room temperature. The fluorescence of all solutions was measured with a $\lambda_{\text {exc }}=360 \mathrm{~nm}$ and the emission was recorded from $400 \mathrm{~nm}$ to $700 \mathrm{~nm}$. For a control experiment, a $\mathrm{NaOH}$ solution was used instead of an NO-donor/NaOH stock solution in order to account for any possible variations in the fluorescence due to minor $\mathrm{pH}$ changes. The result of the control titrations gave identical spectra for all titration points for the $1-150 \mu \mathrm{L}$ range. 


\subsubsection{Fluorescence titration with gradual addition of NO-donor under}

anaerobic and aerobic conditions.

In a typical experiment, a $200 \mu \mathrm{M}$ of solution of (phen) $\mathrm{Re}\left(\mathrm{CH}_{3} \mathrm{CN}\right)(\mathrm{CO})_{2} \mathrm{OSO}_{2} \mathrm{CF}_{3}$ complex was prepared from $1.67 \mathrm{mM}$ stock solution in acetone up to $0.750 \mathrm{~mL}$ of total solution of Re complex. $2.25 \mathrm{~mL}$ of aqueous PBS buffer ( $\mathrm{pH}=7.2$ ) was added. The fluorescence emission was monitored using $\lambda_{\text {exc }}=360$ $\mathrm{nm}$. Then, $3 \mathrm{mM}$ stock solution of MAHMA-NONOate NO-donor in $0.04 \mathrm{M} \mathrm{NaOH}$ was added progressively in $25 \mu \mathrm{L}$ portions up to the total of $300 \mu \mathrm{L}$. After each addition, the solution was left to react for 10 minutes (approximately 3 MAHMA NONOate half-lives) at room temperature before measuring fluorescence using the same parameters as above mentioned.

In another cuvette, another $3000 \mu \mathrm{L}$ of (phen) $\mathrm{Re}\left(\mathrm{CH}_{3} \mathrm{CN}\right)(\mathrm{CO})_{2} \mathrm{OSO}_{2} \mathrm{CF}_{3}$ was prepared in the same acetone/buffer system (see above); this solution was bubbled with nitrogen for 30 minutes. The fluorescence titration was performed under the same conditions mentioned above.

\subsection{RESULTS}

\subsubsection{Synthesis of (phen) $\operatorname{Re}\left(\mathrm{CH}_{3} \mathrm{CN}\right)(\mathrm{CO})_{2} \mathrm{OSO}_{2} \mathrm{CF}_{3}(3)$}

Complex 3 was synthesized by thermal substitution of $\mathrm{CO}$ from (phen) $\mathrm{Re}(\mathrm{CO})_{3} \mathrm{SO}_{3} \mathrm{CF}_{3}$. The isolated product was characterized by ${ }^{1} \mathrm{H}$ and ${ }^{13} \mathrm{C}-\mathrm{NMR}$ and FT-IR. 


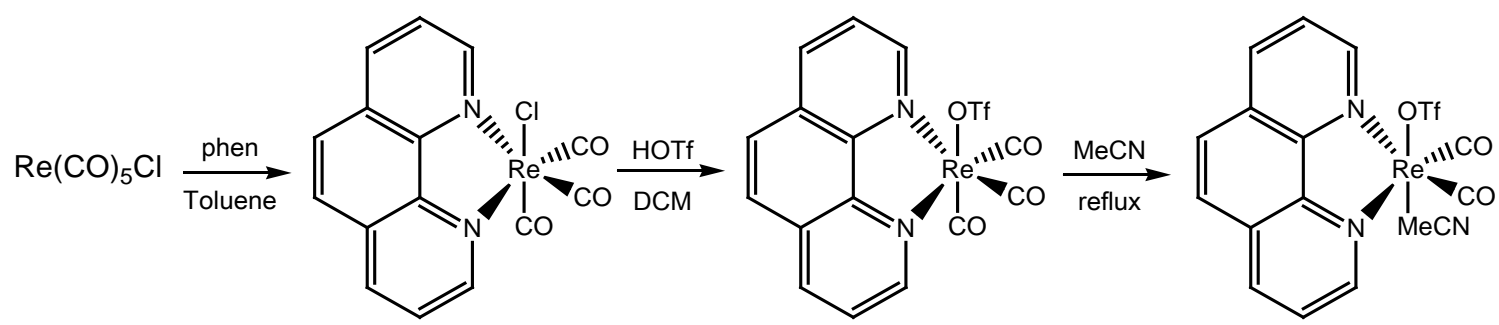

Scheme 4.1 Synthesis of (phen) $\mathrm{Re}\left(\mathrm{CH}_{3} \mathrm{CN}\right)(\mathrm{CO})_{2} \mathrm{OSO}_{2} \mathrm{CF}_{3}$ (3)

\subsubsection{Fluorescence studies with addition of NO-donor}

Complex 3 showed significant fluorescence response to NO addition. The fluorescence spectrum of 3 in acetone/buffer 25/75 v/v shows one emission at $565 \mathrm{~nm}$ ( $\Phi=0.006)$. Figure 4.1 shows the effects on the fluorescence spectrum of 3 upon NO gradual addition, in the form of NO-donor MAHMA-NONOate $(0-150 \mu \mathrm{M})$ dissolved in $0.04 \mathrm{M} \mathrm{NaOH}$. The emission at $562 \mathrm{~nm}$ decreases rapidly upon NO addition and its intensity shows linear dependence with NO-donor concentration, thus allowing NO quantification in the 0-150 $\mu \mathrm{M}$ range (Figure 4.1).

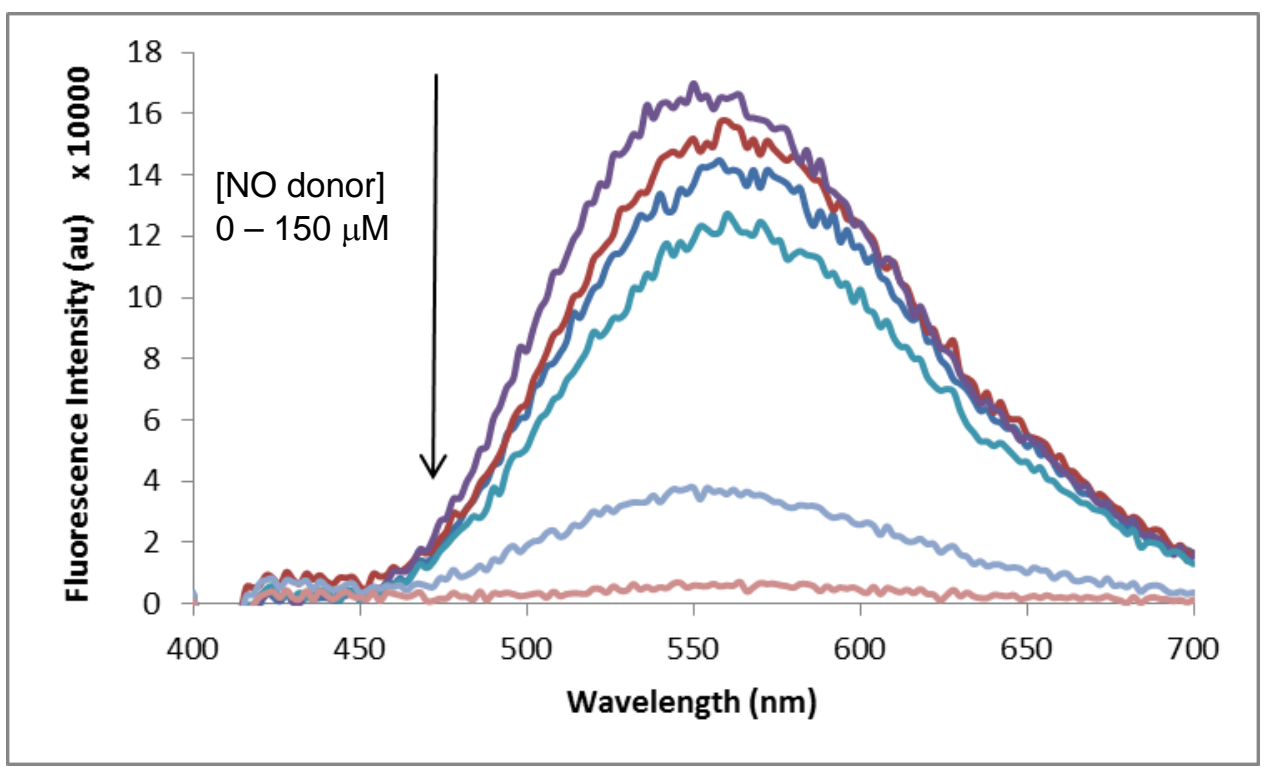

Figure 4.1 Fluorescence Response upon NO-donor addition $(0-150 \mu \mathrm{M})$ to $20 \mu \mathrm{M}$ of Re complex 3 in acetone/PBS aqueous buffer $25 / 75 \mathrm{v} / \mathrm{v},\left(\lambda_{\text {exc }}=360 \mathrm{~nm}\right)$ 


\subsubsection{Fluorescence titration with gradual addition of NO-donor under}

anaerobic and aerobic conditions.

Complex 3 showed significant stability under physiological conditions. Figure 4.2 shows the effects on the fluorescence spectrum of $\mathbf{3}$ upon NO consecutive addition, in the form of NO-donor MAHMA-NONOate $(0-300 \mu \mathrm{M})$ dissolved in $0.04 \mathrm{M} \mathrm{NaOH}$, in $25 \mu \mathrm{L}$ portions after 10 minutes intervals. The emission at $532 \mathrm{~nm}$ decreases rapidly upon NO addition and its intensity shows linear dependence with NO-donor concentration, this experiment was performed under nitrogen to avoid $\mathrm{NO}$ oxidation to $\mathrm{N}_{2} \mathrm{O}_{3}$, in a titration fashion.

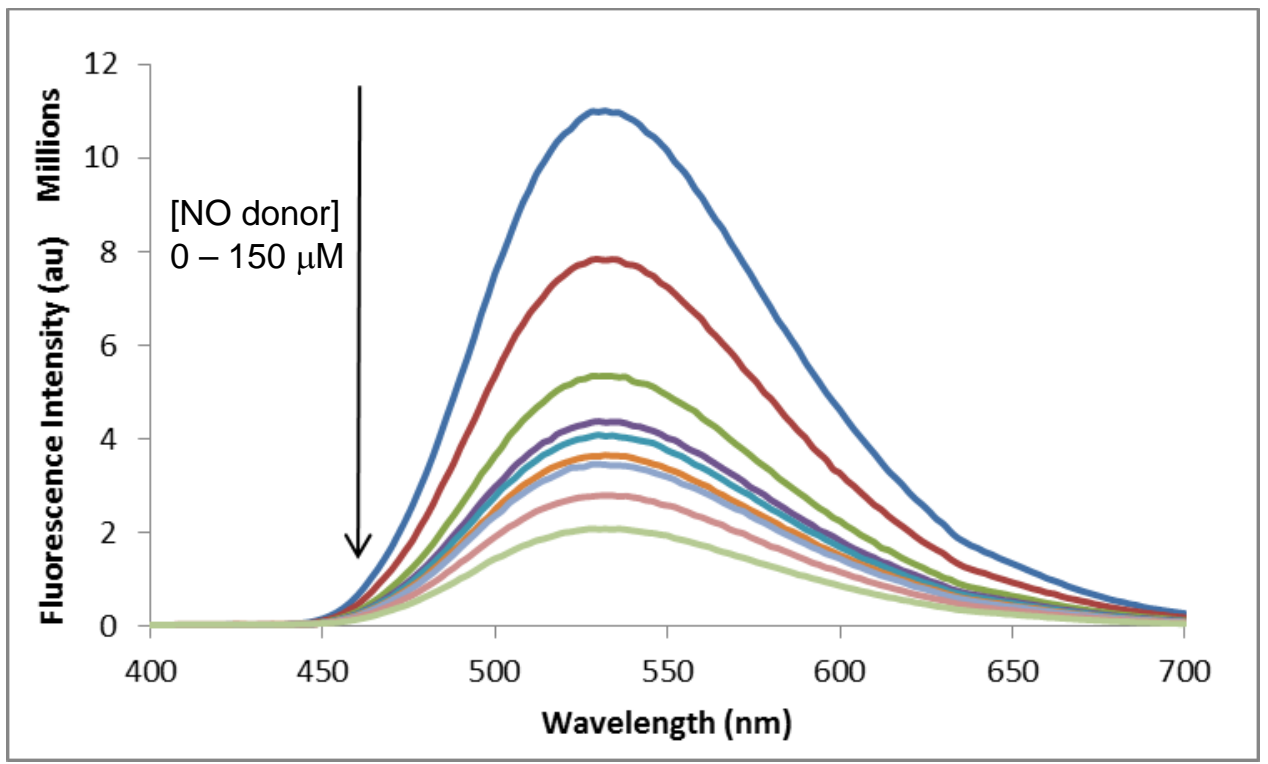

Figure 4.2. Fluorescence Spectra of $50 \mu \mathrm{M} 3$ with NO, acetone/buffer 25:75, $\lambda_{\text {exc }}=360 \mathrm{~nm}$, under nitrogen

Figure 4.3 shows the effects on the fluorescence spectrum of $\mathbf{1}$ upon NO consecutive addition, in the form of NO-donor MAHMA-NONOate $(0-300 \mu \mathrm{M})$ dissolved in $0.04 \mathrm{M} \mathrm{NaOH}$, in $25 \mu \mathrm{L}$ portions after 10 minutes intervals. The emission at 532 nm decreases rapidly upon NO addition and its intensity shows linear dependence 
with NO-donor concentration, this experiment was performed under air, in a titration fashion; some NO got consumed by oxidation. The intensity of the titration performed under air is $30 \%$ lower than under nitrogen, as exhibit in Figure 4.3; and the response towards NO addition is lower in the same extend, that can be explained by oxidation of the real substrate $\mathrm{NO}$ to $\mathrm{NO}_{2}$, and this second one cannot be used as a substrate for this Re complex.

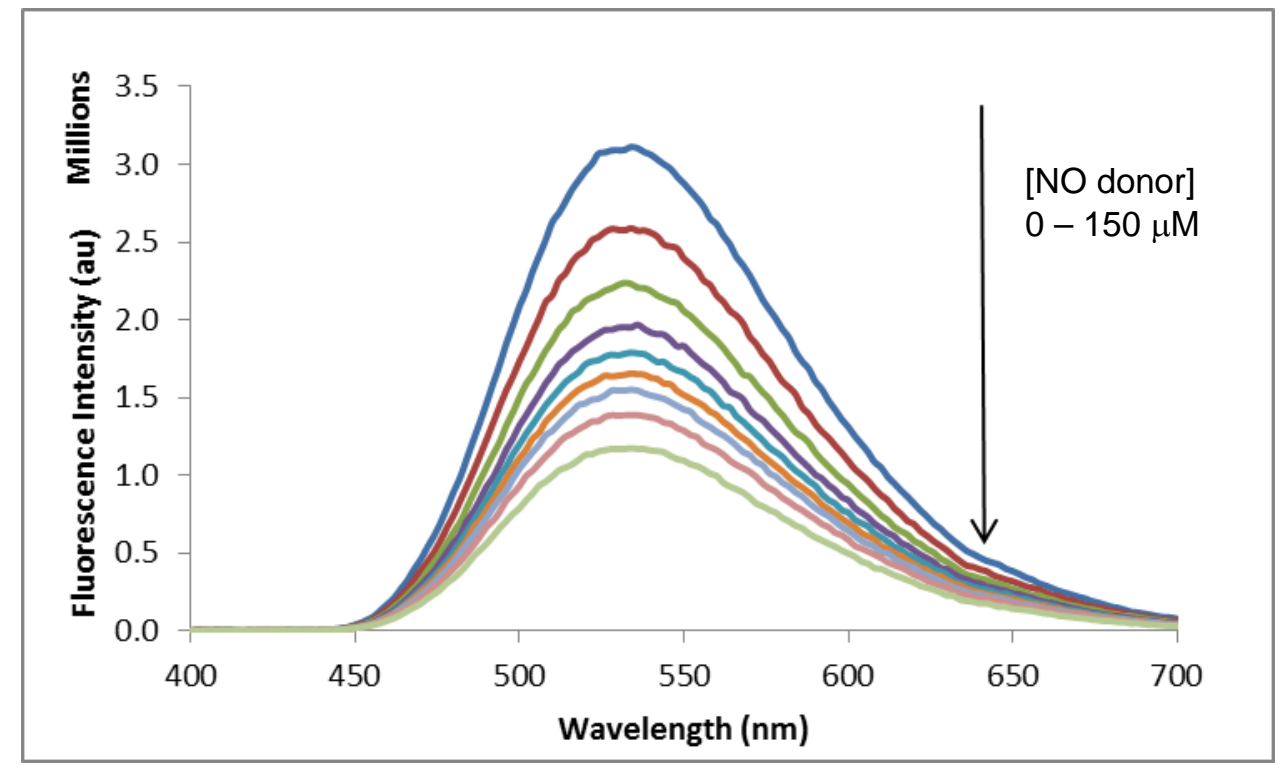

Figure 4.3. Fluorescence Spectra of $50 \mu \mathrm{M} 6$ with NO, acetone/buffer 25:75,

$$
\lambda_{\text {exc }}=360 \mathrm{~nm}
$$

The fluorescence titration comparison plot (Figure 4.4) of I532 vs [NO-donor] under nitrogen (Figure 4.2) and under air (Figure 4.3) shows the similarity of responses in the two experiments, and also higher sensitivity in lower concentrations of NO under anaerobic conditions. Thus, it can be concluded that there is no need for an oxidant in order for the reaction to occur. This result is indicative of direct interaction of the dye with NO and not with NO-derived oxidized products. 
${ }^{19}$ F-NMR after NO-donor reaction showed no signals. It can be concluded that $\mathrm{CF}_{3} \mathrm{SO}_{3}{ }^{-}$got dissociated after reaction with $\mathrm{NO}$; this proves one part of the mechanism.

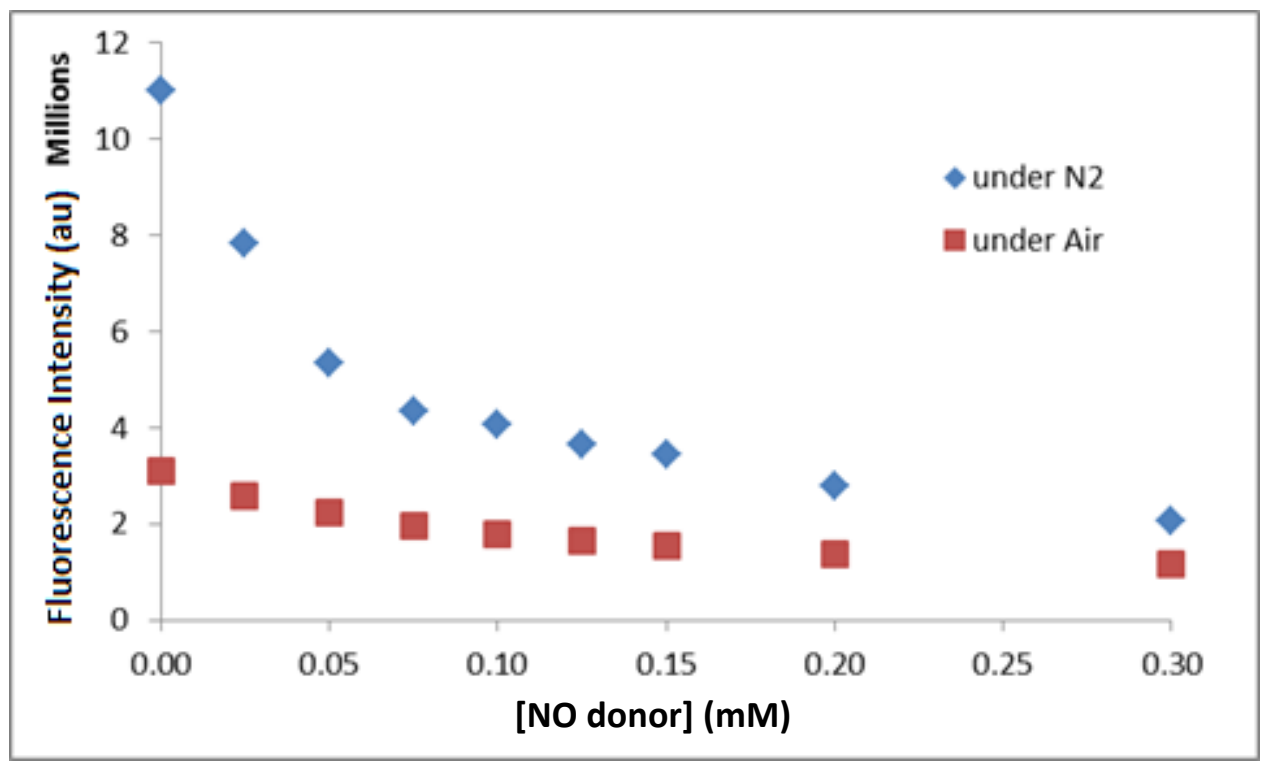

Figure 4.4 Fluorescence Spectra of $350 \mu \mathrm{M}$ with NO, comparison nitrogen vs air at $532 \mathrm{~nm}$.

\subsection{CONCLUSION}

The new complex (phen) $\mathrm{Re}\left(\mathrm{CH}_{3} \mathrm{CN}\right)(\mathrm{CO})_{2} \mathrm{OSO}_{2} \mathrm{CF}_{3}$ has been synthesized and characterized. This complex can react with NO under physiological conditions, producing a quenching in fluorescence that can be monitored.

\subsection{REFERENCES}

1. Kavalllieratos, K; Tsoukias, N. M.; Lozano-Lewis, L. I. "Organometallic dyes for NO detection and imaging". Issued Patent US 8,906,694 B2. Dec. 9, 2014.

2. Lima Barbosa, A. S., Werlé, C., Oliva Colunga, C. O., Franco Rodríguez, C., Toscano, R. A., Le Lagadec, R., Pfeffer, M. Inorg. Chem., 2015, 54, 7617-7626.

3. Boff, B., Ali, M., Alexandrova, L., Espinosa-Jalapa, N. A., Saavedra-Díaz, R. O., Le Lagadec, R., Pfeffer, M. Organometallics, 2013, 32, 5092-5097.

4. Lo, K. K - W., Hui, W - K. Inorg. Chem., 2005, 44, 1992.

5. Ko, C - C., Ng, C - O., Yiu, S - M. Organometallics, 2012, 31, 7074-7084. 
6. (a) Stephenson, K. A.; Banerjee, S. R.; Besenger, T.; Sogbein, O. O.; Levadala, M. K.; McFarlane, N.; Lemon, J. A.; Boreham, D. R.; Maresca, K. P.; Brennan, J. D.; Babich, J. W.; Zubieta, J.; Valliant, J. F. J. Am. Chem. Soc. 2004, 126, 8598; (b) Ferri, E.; Donghi, D.; Panigati, M.; Prencipe, G.; D’Alfonso, L.; Zanoni, I.; Baldoli, C.; Maiorana, S.; D’Alfonso, G.; Licandro, E. Chem. Commun. 2010, 46, 6255

7. Fernández-Moreira, V.; Thorp-Greenwood, F. L.; Amoroso, A. J.; Cable, J.; Court, J. B.; Gray, V.; Hayes, A. J.; Jenkins, R. L.; Kariuki, B. M.; Lloyd, D.; Millet, C. O.; Williams, C. Ff.; Coogan, M. P. Org. Biomol. Chem. 2010, 8, 3888.

8. Ferraudi et al. Dalton, 2007, 2020. Wrighton \& Morse. JACS, 1974, 96, 998. Meyer. JPC, 1983, 87, 952.

9. (a) Geoffroy, G. L.; Wrighton, M. S. Organometallic Photochemistry; Academic Press: New York, 1979; (b) Lees, A. J. Chem. Rev. 1987, 87, 711-743. 


\section{5. $\alpha$-DIIMINE RHENIUM COMPLEXES, AS COLORIMETRIC SENSORS FOR NO and $\mathrm{CN}^{-}$.}

\subsection{ABSTRACT}

The complexes

$$
\text { (Xy-DAD)Re(THF)(CO) } 2 \text { OTf }
$$

(8),

(Xy-

DAD) $\mathrm{Re}\left(\mathrm{CH}_{3} \mathrm{CN}\right)(\mathrm{CO})_{2} \mathrm{OTf}$

(9),

(iPr-DAD)Re(THF)(CO) $)_{2} \mathrm{OTf} \quad(\mathbf{1 0})$, and (iPr-

DAD) $\mathrm{Re}\left(\mathrm{CH}_{3} \mathrm{CN}\right)(\mathrm{CO})_{2} \mathrm{OTf}$

obtained photochemically from (Xy-

$\mathrm{DAD}) \operatorname{Re}(\mathrm{CO})_{3} \mathrm{OTf}(5)$ and (iPr-DAD) $\mathrm{Re}(\mathrm{CO})_{3} \mathrm{OTf}(7)$ respectively show colorimetric response upon $\mathrm{NO}$ and $\mathrm{CN}^{-}$addition, which was added in the form of NO-donor $\mathrm{Z}-1-\mathrm{N}$ methyl-N-[6-(N-methylammoniohexyl)amino]diazen-1-ium-1,2-diolate

(MAHMANONOate) and in the form of NaCN. Addition of 10-100 $\mu \mathrm{M}$ of NO-donor into a $50 \mu \mathrm{M}$ solution of $\mathbf{8 , ~ 9 , ~ 1 0 , ~ a n d ~} \mathbf{1 1}$ in 50\% v/v acetone/phosphate saline (PBS) buffer gave hypsochromic shift from $478 \mathrm{~nm}$ to $408 \mathrm{~nm}$ upon NO addition in the case of complex 7, decreased the signal at $500 \mathrm{~nm}$ upon NO addition in the case of complex 8, increased the signal at $470 \mathrm{~nm}$ upon $\mathrm{CN}^{-}$addition in the case of complex $\mathbf{9}$, and hypsochromic shift from $500 \mathrm{~nm}$ to $348 \mathrm{~nm}$ upon $\mathrm{CN}^{-}$addition in the case of complex 10 . The ${ }^{1} \mathrm{H}-\mathrm{NMR}$ and ${ }^{19} \mathrm{~F}$-NMR spectra after reaction with $\mathrm{CN}^{-}$and NO showed characteristic resonances for non-coordinated triflate, THF, and $\mathrm{CH}_{3} \mathrm{CN}$, suggesting that the organometallic product formed upon $\mathrm{NO}$ and $\mathrm{CN}^{-}$release both these ligands upon reaction with the respective substrate. This project tries to explore the ability of rhenium complexes to bind to $\mathrm{NO}^{1}$ and this change in the coordination sphere may produce an optical change that may be quantifiable.

\subsection{INTRODUCTION}


Nitric oxide has several functions of particular importance for human health. ${ }^{2,3}$ It is now recognized as a key signaling molecule in the cardiovascular system and is also called endothelial derived relaxation factor (EDRF), ${ }^{4}$ as NO regulates blood pressure by relaxing blood capillaries, veins and arteries. Several transition metal nitrosyl complexes have been reported, ${ }^{5}$ including some $\mathrm{Re}(\mathrm{I})$ complexes. ${ }^{6}$ Therefore, late transition metal chemistry should be explored for NO radical binding.

The 1,4-Diazabutadienes (DAD or DAB) are ligands where intramolecular electron transfer can be studied. These intramolecular electron transfers lead to valence tautomerization (VT). 1,4-diazabutadienes have two redox centers, which create the possibility of a bistable electronic state. ${ }^{7}$ Coordinating transition metals to non-innocent redox ligands may generate bistable electronic complexes, as shown in Scheme 5.1.

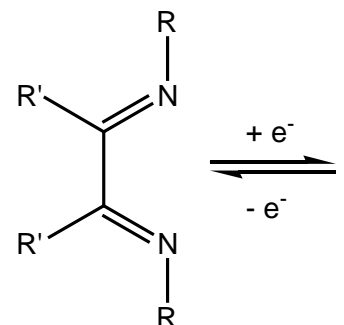

$\mathbf{L}^{\mathrm{R}} \mathbf{R}^{\prime}{ }_{2}$<smiles></smiles>

$M^{+n}\left[L^{R} R_{2}^{\prime}\right]$

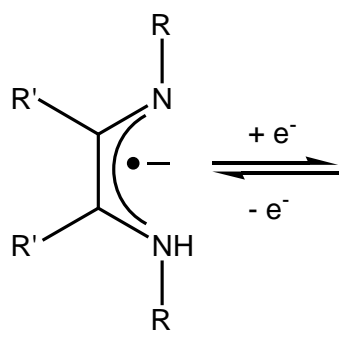

$L^{R} R_{2}^{\prime}$<smiles>[R][N]C([R])=C([R])N[R]</smiles>

$L^{R} R_{2}{ }^{2-}$

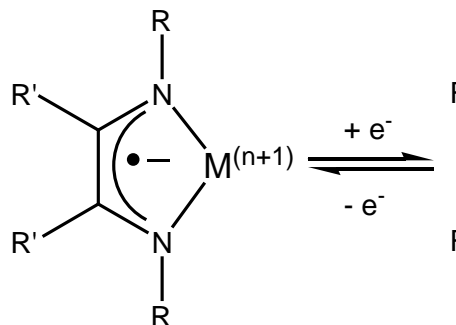

$M^{(n+1)}\left[L^{R} R_{2}^{\prime}\right]^{\cdot-}$<smiles>[R][R11]1c([R])c([R])c([R])n1[R]</smiles>

$M^{(n+2)}\left[L^{R} R_{2}{ }^{2-}\right]$

Scheme 5.1. Valence tautomerization (VT) produced by bistable electron state DAD. 
Another possibility of "distortion" is "back-bonding" from the metal d-orbitals to the empty LUMO $\pi^{*}$ of the diamine ligand. ${ }^{8} \quad$ There are complexes with osmium, ruthenium and rhodium; ${ }^{7}$ but rhenium chemistry with DAD has been barely explored.

The synthesis of metal-DAD complexes can be summarized as substitution from metal halides or metal carbonyls with the DAD ligand, or by chelating ligand substitution, or by Schiff-base condensation. ${ }^{9}$

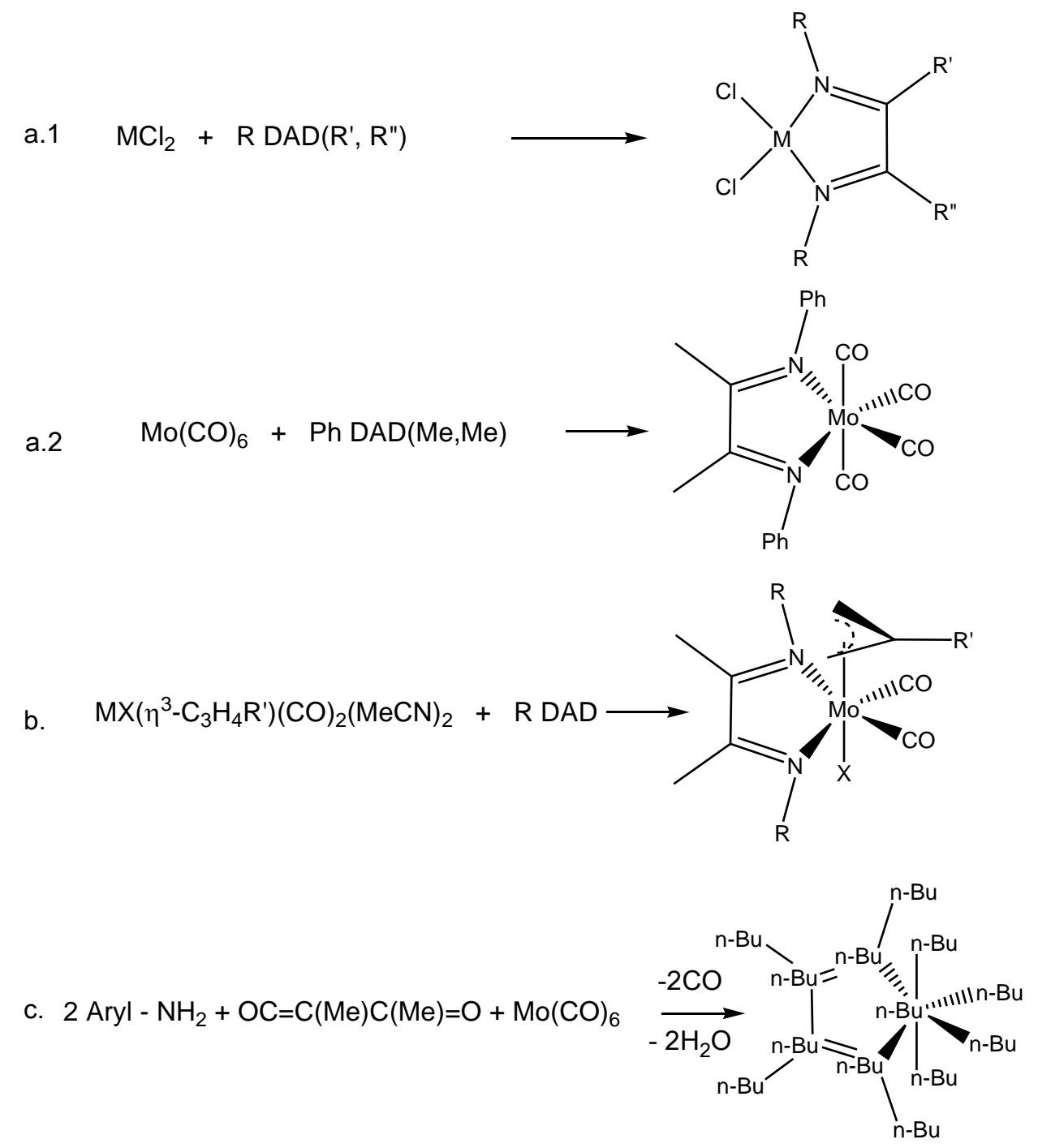

Scheme 5.2 Summary of synthesis of metal-DAD complexes: a. from metal halide or carbonyl; b. from chelating ligand substitution; c. from Schiff-base condensation. 
In prior work (Chapter 2- 4), it was shown that (phen) $\mathrm{Re}\left(\mathrm{PPh}_{3}\right)(\mathrm{CO})_{2} \mathrm{OSO}_{2} \mathrm{CF}_{3}$, (phen) $\mathrm{Re}(\mathrm{THF})(\mathrm{CO})_{2} \mathrm{OSO}_{2} \mathrm{CF}_{3}$, and (phen) $\mathrm{Re}\left(\mathrm{CH}_{3} \mathrm{CN}\right)(\mathrm{CO})_{2} \mathrm{OSO}_{2} \mathrm{CF}_{3}$ prepared in situ thermally or photochemically from (phen) $\mathrm{Re}(\mathrm{CO})_{3} \mathrm{OSO}_{2} \mathrm{CF}_{3}$, have the ability to react with NO under physiological conditions, producing a fluorescence response. These new DAD-Re-THF and DAD-Re- $\mathrm{CH}_{3} \mathrm{CN}$ analogs $\mathbf{8 ,} \mathbf{9}, 10$ and 11, which are introduced in this Chapter, show colorimetric responses to $\mathrm{NO}$ and $\mathrm{CN}^{-}$addition that can be ascribed to $\mathrm{NO}$ or $\mathrm{CN}^{-}$complexation by the Re center.

\subsection{EXPERIMENTAL PROCEDURES}

\subsubsection{Materials and Methods}

All materials (purchased from Aldrich Chemical Co., ACROS organics, Cayman Chemicals, or Alfa Aesar) were standard reagent grade and were used without further purification, unless otherwise mentioned. Toluene, used for the preparation of $\mathbf{3}$ and $\mathbf{5}$, was distilled and dried before use. PBS buffer $(0.002 \mathrm{M}$ in total phosphate, $\mathrm{pH}=7.2$, $[\mathrm{NaCl}]=0.04 \mathrm{M}$ ) was prepared from $\mathrm{K}_{2} \mathrm{HPO}_{4}, \mathrm{KH}_{2} \mathrm{PO}_{4}$, and $\mathrm{NaCl}$ (all from Fisher Scientific). Water, used for preparation of the buffers and experiments with Nitric oxide, was filtered water $\left(18 \mathrm{M} \Omega \cdot \mathrm{cm}^{-1}\right)$ produced from a Millipore ${ }^{\circledR}$ nanopure diamond lab water system (Barnstead Thermolyne Corporation, Dubuque, IA). Deuterated solvents were purchased from Cambridge Isotope Laboratories Ltd. Photochemical reactions were carried out using a Rayonet photochemical reactor (Southern New England Ultraviolet, model RPR-100), equipped with a cooling fan and 10 phosphor coated low-pressure mercury lamps $\left(\lambda_{\max }=300 \mathrm{~nm}\right.$, light intensity $=5.2 \pm 0.1 \times 106$ photon $\left./ \mathrm{sec} / \mathrm{cm}^{3}\right)$.

Fluorescence Spectra were recorded on a Jobin Yvon Horiba Fluoromax 3 
spectrometer. ${ }^{1} \mathrm{H}$ and ${ }^{13} \mathrm{C}$ NMR spectra were recorded on a 400 Bruker NMR spectrometer and were referenced, using the residual solvent resonances. All chemical shifts, $\delta$, are reported in ppm. FT-IR spectra were recorded with a Perkin Elmer Spectrum 100 FT-IR Spectrometer. UV-Visible spectra were recorded with a Cary 100 Spectrophotometer.

\subsubsection{Synthesis of $(\mathrm{Xy}-\mathrm{DAD}) \operatorname{Re}(\mathrm{CO})_{3} \mathrm{Cl}(4)$ :}

$100.0 \mathrm{mg}$ of $\mathrm{Re}(\mathrm{CO})_{5} \mathrm{Cl}(276 \mu \mathrm{mol})$ and $73.0 \mathrm{mg}$ of $\mathrm{Xy}-\mathrm{DAD}(276 \mu \mathrm{mol})$ were suspended in $25 \mathrm{~mL}$ of distilled anhydrous Toluene (final concentration of $11.0 \mathrm{mM}$ ), under $\mathrm{N}_{2}$ flow. The suspension was refluxed for 2 hours. The volatiles were evaporated to half volume. The solution changed from yellow to dark purple. The bright purple powder product was filtered and was dried in vacuo at $40{ }^{\circ} \mathrm{C} .117 .3 \mathrm{mg},(70 \%) .{ }^{1} \mathrm{H}-\mathrm{NMR}$ $\left(\mathrm{CDCl}_{3}\right): 2.29$ (s, 6H), 2.59 (s, 6H), $7.20\left(\mathrm{~m}, 6 \mathrm{H},{ }^{3} \mathrm{~J}_{\mathrm{H}-\mathrm{H}}=4.5 \mathrm{~Hz}\right), 8.75\left(\mathrm{~d}, 2 \mathrm{H},{ }^{3} \mathrm{~J}_{\mathrm{H}-\mathrm{H}}=6.7\right.$ Hz). ${ }^{19} \mathrm{~F}-\left\{{ }^{1} \mathrm{H}\right\}-\mathrm{NMR}\left(\mathrm{CDCl}_{3}\right):-78.3$ ppm. ${ }^{13} \mathrm{C}-\mathrm{NMR}\left(\mathrm{CDCl}_{3}\right): 18.8$ (s, 2C); 20.1 (s, 2C); 128.1 (s, 2C); 128.3 (s, 2C); 128.8 (s, 2C); 129.6 (s, 2C); 150.3 (s, 1C); 166.6 (s, 1C). FT-IR (cm ${ }^{-1}$ ): 2021 (vco, vs), 1931 (vco, vs), 1885 (vco, vs), 1637, 1499, 1468, 1382, 1265, 1185, 1098, 1029, 771. UV-Vis (in ethanol): $\lambda \max =440 \mathrm{~nm}-$ br $\left(\varepsilon=8075 \mathrm{~mol}^{-1} \mathrm{~cm}^{-1}\right)$. Anal. Calc. for $\mathrm{C}_{21} \mathrm{H}_{20} \mathrm{ClN}_{2} \mathrm{O}_{3} \mathrm{Re}$ : C 44.24\%, H 3.37\%, N 4.91\%. Found: C 43.96\%, H 3.37\%, N 4.89\%.

\subsubsection{Synthesis of $(\mathrm{Xy}-\mathrm{DAD}) \operatorname{Re}(\mathrm{CO})_{3} \mathrm{OSO}_{2} \mathrm{CF}_{3}(5)$ :}

$50.0 \mathrm{mg}$ of $(\mathrm{Xy}-\mathrm{DAD}) \mathrm{Re}(\mathrm{CO})_{3} \mathrm{Cl}$ (3) $(87.7 \mu \mathrm{mol})$ and $20 \mathrm{~mL}$ of dichloromethane (4.3 $\mu \mathrm{M}$ of concentration) was placed in a $50 \mathrm{~mL}$ round bottom flask under $\mathrm{N}_{2}$ flow. $0.15 \mathrm{~mL}$ of triflic acid was added dropwise. The solution was stirred for 2 hours at room temperature. The volatiles were evaporated to $1.0 \mathrm{~mL}$. The product was precipitated at 
room temperature by adding $4.0 \mathrm{~mL}$ of diethyl ether. The bright purple powder product was filtered and was dried in vacuo at $40{ }^{\circ} \mathrm{C} .59 .5 \mathrm{mg}$, (98\%). ${ }^{1} \mathrm{H}-\mathrm{NMR}\left(\mathrm{CDCl}_{3}\right): 2.36$ (s, 6H), 2.39 (s, 6H), $7.23\left(\mathrm{~m}, 6 \mathrm{H},{ }^{3} \mathrm{~J}-\mathrm{H}=4.5 \mathrm{~Hz}\right), 8.77$ (s, 2H). ${ }^{19} \mathrm{~F}-\left\{{ }^{1} \mathrm{H}\right\}-\mathrm{NMR}\left(\mathrm{CDCl}_{3}\right)$ : -78.2 ppm. ${ }^{13} \mathrm{C}-\mathrm{NMR}\left(\mathrm{CDCl}_{3}\right.$ ): 18.6 (s, 2C); 18.9 (s, 2C); 128.1 (s, 2C); 128.7 (s, 2C); 129.2 (s, 2C); 129.7 (s, 2C); 168.9.3 (s, 1C); 171.6 (s, 1C). FT-IR (cm $\left.{ }^{-1}\right): 2038$ (vco, vs), 1951 (vco, vs), 1909 (vco, vs), 1313, 1233, 1204, 1190, 1005, 773, 624. Anal. Calc. for $\mathrm{C}_{21} \mathrm{H}_{20} \mathrm{~F}_{3} \mathrm{~N}_{2} \mathrm{O}_{6} \mathrm{ReS}$ : C 38.59\%, H 3.09\%, N 4.09\%. Found: C 38.25\%, H 2.82\%, N $3.99 \%$.

\subsubsection{Synthesis of (i-Pr-DAD) $\operatorname{Re}(\mathrm{CO})_{3} \mathrm{Cl}(6)$ :}

$100.0 \mathrm{mg}$ of $\operatorname{Re}(\mathrm{CO}){ }_{5} \mathrm{Cl}(276 \mu \mathrm{mol})$ and $105.0 \mathrm{mg}$ of iPr-DAD $(276 \mu \mathrm{mol})$ were suspended in $25 \mathrm{~mL}$ of distilled, anhydrous Toluene (final concentration of $11.0 \mathrm{mM}$ ), under $\mathrm{N}_{2}$ flow. The suspension was refluxed for $2 \mathrm{~h}$. The volatiles were evaporated to half volume. The solution changed from yellow to dark purple. The bright purple powder product was filtered and was dried in vacuo at $40{ }^{\circ} \mathrm{C} .123 .0 \mathrm{mg}$, (55\%). ${ }^{1} \mathrm{H}-\mathrm{NMR}$ $\left(\mathrm{CDCl}_{3}\right): 1.16\left(\mathrm{dd}, 12 \mathrm{H},{ }^{3} \mathrm{~J}_{\mathrm{H}-\mathrm{H}}=14.5 \mathrm{~Hz},{ }^{3} \mathrm{~J}_{\mathrm{H}-\mathrm{H}}=6.8 \mathrm{~Hz}\right), 1.36\left(\mathrm{~d}, 12 \mathrm{H},{ }^{3} \mathrm{~J}_{\mathrm{H}-\mathrm{H}}=6.8 \mathrm{~Hz}\right)$, $2.80\left(\mathrm{~m}, 2 \mathrm{H},{ }^{3} \mathrm{~J}_{\mathrm{H}-\mathrm{H}}=6.7 \mathrm{~Hz}\right), 3.92\left(\mathrm{~m}, 2 \mathrm{H},{ }^{3} \mathrm{~J}_{\mathrm{H}-\mathrm{H}}=6.7 \mathrm{~Hz}\right), 7.72\left(\mathrm{~m}, 6 \mathrm{H},{ }^{3} \mathrm{~J}_{\mathrm{H}-\mathrm{H}}=4.5 \mathrm{~Hz}\right)$, 8.70 (s, 2H). ${ }^{19} \mathrm{~F}-\left\{{ }^{1} \mathrm{H}\right\}-\mathrm{NMR}\left(\mathrm{CDCl}_{3}\right):-78.3$ ppm. ${ }^{13} \mathrm{C}-\mathrm{NMR}\left(\mathrm{CDCl}_{3}\right): 22.8$ (s, 2C); 22.9 (s, 2C); 26.2 (s, 2C); 26.8 (s, 2C); 28.0 (s, 2C); 28.2 (s, 2C); 123.8 (s, 2C); 124.9 (s, 2C); 128.8 (s, 2C); 139.2 (s, 2C); 147.8 (s, 1C); 166.7 (s, 1C). FT-IR (cm): 2025 (vco, vs), 1934 (vco, vs), 1916 (vco, vs), 1501, 1464, 1441, 1362, 1355, 1321, 1170, 1102, 796, 752, 638. Anal. Calc. for $\mathrm{C}_{21} \mathrm{H}_{20} \mathrm{ClN}_{2} \mathrm{O}_{3}$ Re: C 51.05\%, H 5.31\%, N 4.10\%. Found: C 50.86\%, H 5.15\%, N 4.10\%.

\subsubsection{Synthesis of (i-Pr-DAD) Re(CO) $)_{3} \mathrm{OSO}_{2} \mathrm{CF}_{3}(7)$ :}


$50.0 \mathrm{mg}$ of (i-Pr-DAD) $\operatorname{Re}(\mathrm{CO}){ }_{3} \mathrm{Cl}(5)(87.7 \mu \mathrm{mol})$ and $20 \mathrm{~mL}$ of dichloromethane (4.3 $\mathrm{mM}$ of concentration) was placed in a $50 \mathrm{~mL}$ round bottom flask under $\mathrm{N}_{2}$ flow. $0.15 \mathrm{~mL}$ of triflic acid was added dropwise. The solution was stirred for 2 hours at room temperature. The volatiles were evaporated to $1.0 \mathrm{~mL}$. The product was precipitated at room temperature by adding $4.0 \mathrm{~mL}$ of diethyl ether. The bright purple powder product was filtered and was dried in vacuo at $40{ }^{\circ} \mathrm{C} .59 .5 \mathrm{mg}$, (98\%). ${ }^{1} \mathrm{H}-\mathrm{NMR}\left(\mathrm{CDCl}_{3}\right): 1.15$ (dd, $\left.12 \mathrm{H},{ }^{3} \mathrm{~J}_{\mathrm{H}-\mathrm{H}}=67.4 \mathrm{~Hz},{ }^{3} \mathrm{~J}_{\mathrm{H}-\mathrm{H}}=6.7 \mathrm{~Hz}\right), 1.38\left(\mathrm{~d}, 12 \mathrm{H},{ }^{3} \mathrm{~J}_{\mathrm{H}-\mathrm{H}}=18.3 \mathrm{~Hz},{ }^{3} \mathrm{~J}_{\mathrm{H}-\mathrm{H}}=6.7 \mathrm{~Hz}\right)$, $2.77\left(\mathrm{~m}, 2 \mathrm{H},{ }^{3} \mathrm{~J}_{\mathrm{H}-\mathrm{H}}=6.7 \mathrm{~Hz}\right), 3.16\left(\mathrm{~m}, 2 \mathrm{H},{ }^{3} \mathrm{~J}_{\mathrm{H}-\mathrm{H}}=6.7 \mathrm{~Hz}\right), 7.29\left(\mathrm{~m}, 6 \mathrm{H},{ }^{3} \mathrm{~J}_{\mathrm{H}-\mathrm{H}}=3.5 \mathrm{~Hz}\right)$, $7.37\left(\mathrm{~m}, \quad 6 \mathrm{H},{ }^{3} \mathrm{~J}_{\mathrm{H}-\mathrm{H}}=3.5 \mathrm{~Hz}\right), 8.75(\mathrm{~s}, \quad 2 \mathrm{H}) . \quad{ }^{19} \mathrm{~F}-\left\{{ }^{1} \mathrm{H}\right\}-\mathrm{NMR}\left(\mathrm{CDCl}_{3}\right):-78.2$ ppm. $\quad{ }^{13} \mathrm{C}-\mathrm{NMR}\left(\mathrm{CDCl}_{3}\right)$ ) 22.2 (s, 2C); 23.2 (s, 2C); 26.3 (s, 2C); 26.9 (s, 2C); 28.3 (s, 2C); 28.4 (s, 2C); 124.3 (s, 2C); 124.9 (s, 2C); 129.4 (s, 2C); 139.2 (s, 2C); 141.0 (s, 2C); 146.9 (s, 1C); 169.0 (s, 1C). FT-IR ( $\left.\mathrm{cm}^{-1}\right)$ : 2038 ( $\left.v_{\mathrm{co}}, \mathrm{vs}\right), 1953$ ( $\left.v_{\mathrm{co}}, \mathrm{vs}\right), 1922$ (vco, vs), 1510, 1325, 1316, 1235, 1202, 1192, 1001, 796, 750, 633.

\subsubsection{Photochemical substitution reactions:}

All the solutions were irradiated with a Rayonet photochemical reactor (Southern New England Ultraviolet, model RPR-100), equipped with a cooling fan and 10 phosphor coated low-pressure mercury lamps $\left(\lambda_{\max }=300 \mathrm{~nm}\right.$, light intensity $=5.2 \pm 0.1 \times 10^{6}$ photon/sec/ $\mathrm{cm}^{3}$ ). After 75 minutes of irradiation, the colors of the solutions changed to darker colors. Unfortunately, all of the organometallic complexes could not be isolated and it had to be prepared in situ.

\subsubsection{Synthesis of $(\mathrm{Xy}-\mathrm{DAD}) \mathrm{Re}(\mathrm{THF})(\mathrm{CO})_{2} \mathrm{OSO}_{2} \mathrm{CF}_{3}(8)$ :}

(Xy-DAD)Re(CO) ${ }_{3} \mathrm{OSO}_{2} \mathrm{CF}_{3}(4)(1 \mathrm{mg}, 1.46 \mu \mathrm{mol}) 1.00 \mathrm{~mL}$ of anhydrous THF (final concentration $1.46 \mathrm{mM}$ ) were placed in a NMR tube. The solution was irradiated 
for 75 minutes at room temperature. This solution was used without further treatment. ${ }^{1} \mathrm{H}-\mathrm{NMR}\left(\mathrm{CDCl}_{3}\right): 2.24(\mathrm{~s}, 6 \mathrm{H}), 2.36$ (s, 6H), $7.10\left(\mathrm{~m}, 6 \mathrm{H},{ }^{3} \mathrm{~J}_{\mathrm{H}-\mathrm{H}}=3.4 \mathrm{~Hz}\right)$, $7.12(\mathrm{~m}, 6 \mathrm{H}), 9.04(\mathrm{~s}, 2 \mathrm{H}) . \quad{ }^{19} \mathrm{~F}-\left\{{ }^{1} \mathrm{H}\right\}-\mathrm{NMR}\left(\mathrm{CDCl}_{3}\right):-78.1 \mathrm{ppm} . \quad$ FT-IR $\left(\mathrm{cm}^{-1}\right): 2036$ (vco, vs), 1945 (vco, vs), 1918 (vco, vs), 1720 (vco-o, vs), 1461, 1237, 1177, 1143, 1091, 1030, 994, 921, 775, 638, 614.

\subsubsection{Synthesis of $(\mathrm{Xy}-\mathrm{DAD}) \mathrm{Re}\left(\mathrm{CH}_{3} \mathrm{CN}\right)(\mathrm{CO})_{2} \mathrm{OSO}_{2} \mathrm{CF}_{3}(9)$ :}

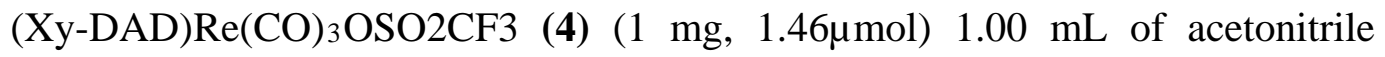
(final concentration $1.46 \mathrm{mM}$ ) were placed in a NMR tube. The solution was irradiated for 75 minutes at room temperature. This solution was used without further treatment. ${ }^{1} \mathrm{H}-\mathrm{NMR}\left(\mathrm{CDCl}_{3}\right): 2.32$ (s, 6H), 2.41 (s, 6H), $7.26(\mathrm{~m}, 6 \mathrm{H}), 7.26(\mathrm{~m}, 6 \mathrm{H})$, $8.86(\mathrm{~s}, 2 \mathrm{H}) . \quad{ }^{19} \mathrm{~F}-\left\{{ }^{1} \mathrm{H}\right\}-\mathrm{NMR}\left(\mathrm{CDCl}_{3}\right):-78.2 \mathrm{ppm} . \quad$ FT-IR $\left(\mathrm{cm}^{-1}\right): 2307\left(v_{\mathrm{cN}}, \mathrm{w}\right), 2040$ (vco, vs), 1939 (vco, vs-br), 1470, 1385, 1327, 1262, 1225, 1200, 1160, 1098, 1030, 775, 701, 638.

\subsubsection{Synthesis of (i-Pr-DAD) $\operatorname{Re}\left(\mathrm{CH}_{3} \mathrm{CN}\right)(\mathrm{CO})_{2} \mathrm{OSO}_{2} \mathrm{CF}_{3}(10)$ :}

(i-Pr-DAD)Re(CO) $)_{3} \mathrm{OSO} 2 \mathrm{CF} 3$ (6) $(1 \mathrm{mg}, 1.26 \mu \mathrm{mol}) 1.00 \mathrm{~mL}$ of anhydrous tetrahydrofurane (final concentration $1.46 \mathrm{mM}$ ) were placed in a NMR tube. The solution was irradiated for 75 minutes at room temperature. This solution was used without further treatment. ${ }^{1} \mathrm{H}-\mathrm{NMR}\left(\mathrm{CDCl}_{3}\right): 0.96\left(\mathrm{dd}, 12 \mathrm{H},{ }^{3} \mathrm{~J}_{\mathrm{H}-\mathrm{H}}=6.6 \mathrm{~Hz},\right), 1.15\left(\mathrm{dd}, 12 \mathrm{H},{ }^{3} \mathrm{~J}_{\mathrm{H}-}\right.$ $\mathrm{H}=6.5 \mathrm{~Hz}), 1.27\left(\mathrm{dd}, 12 \mathrm{H},{ }^{3} \mathrm{~J}_{\mathrm{H}-\mathrm{H}}=6.4 \mathrm{~Hz}\right), 1.28\left(\mathrm{~d}, 12 \mathrm{H},{ }^{3} \mathrm{~J}_{\mathrm{H}-\mathrm{H}}=4.5 \mathrm{~Hz}\right), 1.33(\mathrm{~m}$, $\left.2 \mathrm{H},{ }^{3} \mathrm{~J}_{\mathrm{H}-\mathrm{H}}=5.4 \mathrm{~Hz}\right), 3.13\left(\mathrm{~m}, 2 \mathrm{H},{ }^{3} \mathrm{~J}_{\mathrm{H}-\mathrm{H}}=6.6 \mathrm{~Hz}\right), 7.27(\mathrm{~m}, 3 \mathrm{H}), 7.68(\mathrm{~m}, 3 \mathrm{H}), 9.05(\mathrm{~s}$, 2H). ${ }^{19} \mathrm{~F}-\left\{{ }^{1} \mathrm{H}\right\}-\mathrm{NMR}\left(\mathrm{CDCl}_{3}\right):-78.1$ ppm. FT-IR (cm $\left.{ }^{-1}\right): 2037$ (vco, vs), 1947 (vco, vs), 1922 (vco, vs), 1722 (vco-o, vs), 1460, 1335, 1238, 1174, 1143, 1091, 1032, 989, 919, 802, 756, 637,614. 


\subsubsection{Synthesis of (i-Pr-DAD) $\operatorname{Re}\left(\mathrm{CH}_{3} \mathrm{CN}\right)(\mathrm{CO})_{2} \mathrm{OSO}_{2} \mathrm{CF}_{3}(11)$ :}

(i-Pr-DAD)Re(CO) ${ }_{3} \mathrm{OSO} 2 \mathrm{CF} 3$ (6) $(1 \mathrm{mg}, 1.26 \mu \mathrm{mol}) 1.00 \mathrm{~mL}$ of anhydrous tetrahydrofurane (final concentration $1.46 \mathrm{mM}$ ) were placed in a NMR tube. The solution was irradiated for 75 minutes at room temperature. This solution was used

without further treatment. ${ }^{1} \mathrm{H}-\mathrm{NMR}\left(\mathrm{CDCl}_{3}\right): 1.26\left(\mathrm{dd}, 12 \mathrm{H},{ }^{3} \mathrm{~J}_{\mathrm{H}-\mathrm{H}}=7.0 \mathrm{~Hz},{ }^{3} \mathrm{~J}_{\mathrm{H}-\mathrm{H}}=7.0\right.$ $\mathrm{Hz}), 1.41\left(\mathrm{~d}, 12 \mathrm{H},{ }^{3} \mathrm{~J}_{\mathrm{H}-\mathrm{H}}=9.8 \mathrm{~Hz},{ }^{3} \mathrm{~J}_{\mathrm{H}-\mathrm{H}}=6.9 \mathrm{~Hz}\right), 2.81\left(\mathrm{~m}, 2 \mathrm{H},{ }^{3} \mathrm{~J}_{\mathrm{H}-\mathrm{H}}=6.8 \mathrm{~Hz}\right), 3.07(\mathrm{~m}$, $\left.2 \mathrm{H},{ }^{3} \mathrm{~J}_{\mathrm{H}-\mathrm{H}}=6.8 \mathrm{~Hz}\right), 7.45(\mathrm{~m}, 4 \mathrm{H}), 7.48(\mathrm{~m}, 2 \mathrm{H}), 8.99(\mathrm{~s}, 2 \mathrm{H}) . \quad{ }^{19} \mathrm{~F}-\left\{{ }^{1} \mathrm{H}\right\}-\mathrm{NMR}\left(\mathrm{CDCl}_{3}\right)$ : -78.2 ppm FT-IR $\left(\mathrm{cm}^{-1}\right)$ : $2298\left(v_{\mathrm{CN}}, \mathrm{w}\right), 2041$ ( $\left.v_{\mathrm{co}}, \mathrm{vs}\right), 1942$ ( $\left.v_{\mathrm{CO}}, \mathrm{vs}\right)$, 1722, 1633, 1437, 1279, 1261, 1180, 1118, 1030, 749, 693, 637.

\subsubsection{Reactivity of $\alpha$-diimine complexes with NO-donor:}

The amount of Re-DAD (stock solution in THF or $\mathrm{CH}_{3} \mathrm{CN}$ ) complex solution needed for the final concentration was calculated assuming $4 \mathrm{~mL}$ of final $50 \mu \mathrm{M}$ solution. Up to $2.0 \mathrm{~mL}$ of acetone was added in order to obtain a solution $50 \% \mathrm{v} / \mathrm{v}$ of total organic solvent. Up to $2.0 \mathrm{~mL}$ of aqueous buffer was added in order to obtain $50 \% \mathrm{v} / \mathrm{v}$ aqueous solution total between Phosphate Buffer Saline $\mathrm{pH}=7.4$; and $4 \mathrm{mM}$ NO-donor stock solution was prepared in $0.04 \mathrm{M} \mathrm{NaOH}$; specific amounts were added to achieve a final NO-donor concentration going from 1 to $200 \mu \mathrm{M}$ range. No more than 10 minutes elapsed from the dilution of the $\mathrm{THF} / \mathrm{CH}_{3} \mathrm{CN}$ stock rhenium complex solution to the final addition of NO-donor. The solution was mixed thoroughly in a vortex and sonicated for 90 minutes at room temperature. At higher concentrations of NO-donor, the solutions became clear. The UV-Visible spectra of this set of solutions were recorded from $300 \mathrm{~nm}$ to $650 \mathrm{~nm}$.

\subsubsection{Reactivity of $\alpha$-diimine complexes with $\mathrm{NaCN}$ :}


The amount of Re-DAD (stock solution in THF or $\mathrm{CH}_{3} \mathrm{CN}$ ) complex solution needed for the final concentration was calculated assuming $4 \mathrm{~mL}$ of final $50 \mu \mathrm{M}$ solution. Up to $2.0 \mathrm{~mL}$ of acetone was added in order to obtain a solution $50 \% \mathrm{v} / \mathrm{v}$ of total organic solvent. Up to $2.0 \mathrm{~mL}$ of aqueous buffer was added in order to obtain $50 \% \mathrm{v} / \mathrm{v}$ aqueous solution total between Phosphate Buffer Saline $\mathrm{pH}=7.4$; and $4 \mathrm{mM}$ NaCN stock solution was prepared in DI water; specific amounts were added to achieve a final $\mathrm{NaCN}$ concentration going from 1 to $100 \mu \mathrm{M}$ range. No more than 10 minutes elapsed from the dilution of the THF/ $\mathrm{CH}_{3} \mathrm{CN}$ stock rhenium complex solution to the final addition of NOdonor. The solution was mixed thoroughly in a vortex and sonicated for 90 minutes at room temperature. At higher concentrations of NO-donor, the solutions became yellow. The UV-Visible spectra of this set of solutions were recorded from $300 \mathrm{~nm}$ to $650 \mathrm{~nm}$.

\subsubsection{Comparison studies with other ions:}

The solutions of $4 \mathrm{mM} \mathrm{NaNO}_{2}, \mathrm{NaNO}_{3}, \mathrm{NaClO}_{4}, \mathrm{NaOCl}, \mathrm{H}_{2} \mathrm{O}_{2}$, and $\mathrm{NaCN}$ were

prepared in ultrapure water. ${ }^{1} \mathrm{O}_{2}$ was prepared in situ, mixing same the amounts of $\mathrm{NaOCl}$ and $\mathrm{H}_{2} \mathrm{O}_{2}$. Stock solutions of (Xy-DAD) rhenium complexes were diluted in acetone for preparing $200 \mu \mathrm{M}$ solutions. $2.8 \mathrm{~mL}$ of $0.002 \mathrm{M}$ PBS pH 7.2 was added to 1 $\mathrm{mL}$ of the previous prepared Re solutions. $100 \mu \mathrm{L}$ of PBS was added to the first tube, this tube will be the standard (std). To the rest there were added $100 \mu \mathrm{L}$ of each of the competitive ions and/or species $\left(\mathrm{NO}_{2}^{-}, \mathrm{NO}_{3}^{-}, \mathrm{ClO}_{4}{ }^{-}, \mathrm{ClO}^{-}, \mathrm{H}_{2} \mathrm{O}_{2}, \mathrm{CN}^{-}\right.$, and $\left.{ }^{1} \mathrm{O}_{2}\right)$. After the solutions were allowed to react for $15 \mathrm{~min}$, a UV-Visible spectrum was measured. $100 \mu \mathrm{L}$ of NO-donor were added to each tube and all the solutions were allowed to react for $1 \mathrm{~h}$, a second UV spectrum was recorded.

\subsection{RESULTS}




\subsubsection{Synthesis of $(\mathrm{R}-\mathrm{DAD}) \operatorname{Re}(\mathrm{L})(\mathrm{CO})_{2} \mathrm{OSO}_{2} \mathrm{CF}_{3}(\mathrm{R}=\mathrm{Me}$ or $\mathrm{i}-\mathrm{Pr} ; \mathrm{L}=\mathrm{THF}$ or $\left.\mathrm{CH}_{3} \mathrm{CN}\right)$}

Complexes 8, 9, 10, and 11 were synthesized in situ by photochemical substitution of $\mathrm{CO}$ from (R-DAD) $\mathrm{Re}(\mathrm{CO})_{3} \mathrm{SO}_{3} \mathrm{CF}_{3}$. The products were characterized by ${ }^{1} \mathrm{H}$ and ${ }^{13} \mathrm{C}-\mathrm{NMR}$ and FT-IR.

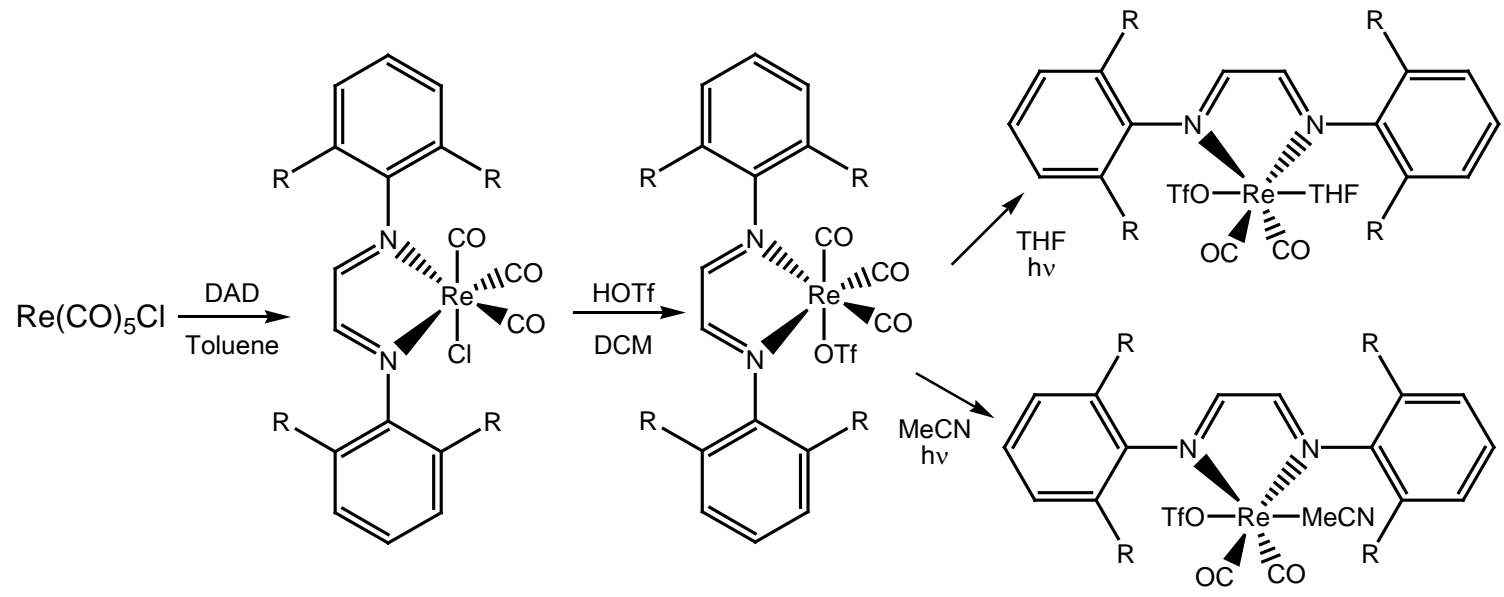

Scheme 5.3 Synthetic path for DAD-rhenium complexes

\subsubsection{Reactivity of $\alpha$-diimine complexes with NO-donor:}

In the case of Figure 5.1, which shows the spectrum UV - Visible of the interaction of (Xy-DAD) $\mathrm{Re}(\mathrm{THF})(\mathrm{CO})_{2} \mathrm{OSO}_{2} \mathrm{CF}_{3}$ (8) with NO-donor, in the form of MAHMA NONOate from $0-200 \mu \mathrm{M}$. It can be seen that the signal decreases at $478 \mathrm{~nm}$ and shifts hypsochromically, meanwhile the absorbance at $408 \mathrm{~nm}$ increases with an isosbestic point around $451 \mathrm{~nm}$. This color change can be observed by the naked eye. 


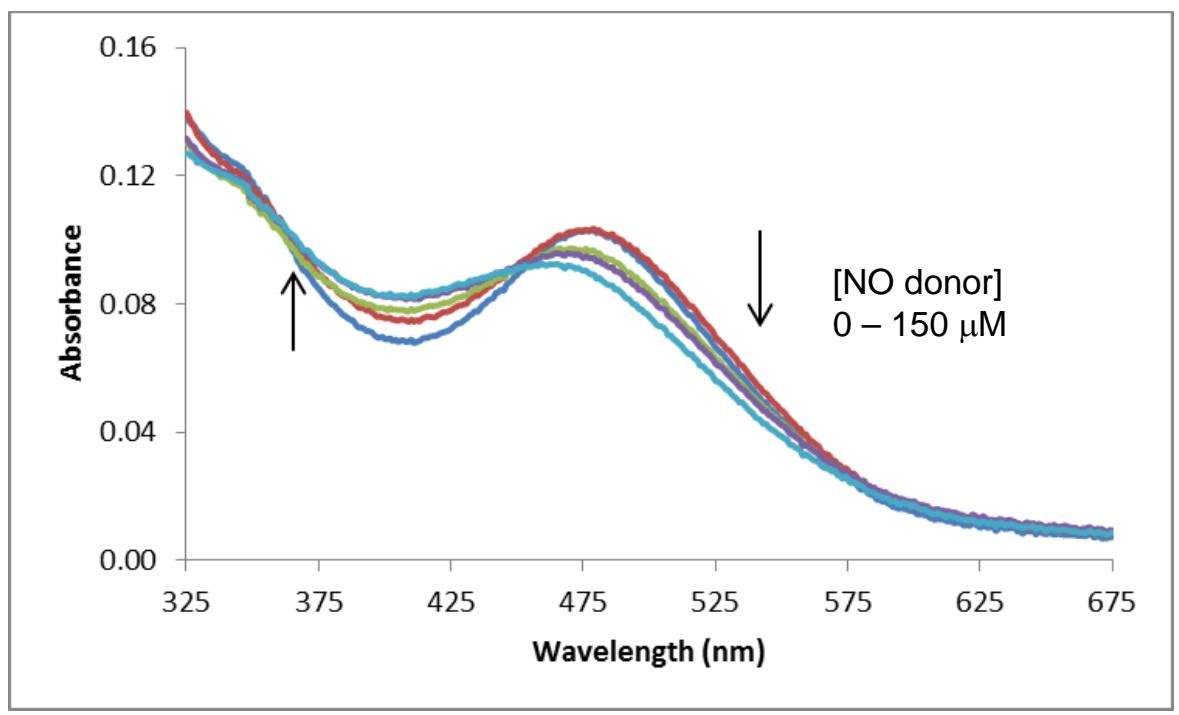

Figure 5.1 Interaction of (Xy-DAD) $\mathrm{Re}(\mathrm{CO})_{2}(\mathrm{THF}) \mathrm{OSO}_{2} \mathrm{CF}_{3}$ with NO-donor, acetone/PBS (1:1)

Complex 8 prepared photochemically on a $1 \mathrm{mg}$ scale (1.67 mM in THF) showed also significant colorimetric response to $\mathrm{NO}$ addition both at $478 \mathrm{~nm}$ (decrease) and at $408 \mathrm{~nm}$ (increase). The UV-Vis spectrum of 8 in THF/acetone/buffer 2/48/50 shows one absoption signal at $478 \mathrm{~nm}$. Figure 5.2 shows the plot of the local maxima and minima on the UV-Visible spectrum of $\mathbf{8}$ upon NO gradual addition, in the form of NO-donor MAHMA-NONOate $(0-200 \mu \mathrm{M})$ dissolved in $0.04 \mathrm{M} \mathrm{NaOH}$. The graph also shows the ratio of the absorbance at $408 \mathrm{~nm}$ vs the absorbance at $478 \mathrm{~nm}$. This plot shows a linear dependency at lower NO-donor concentrations. 


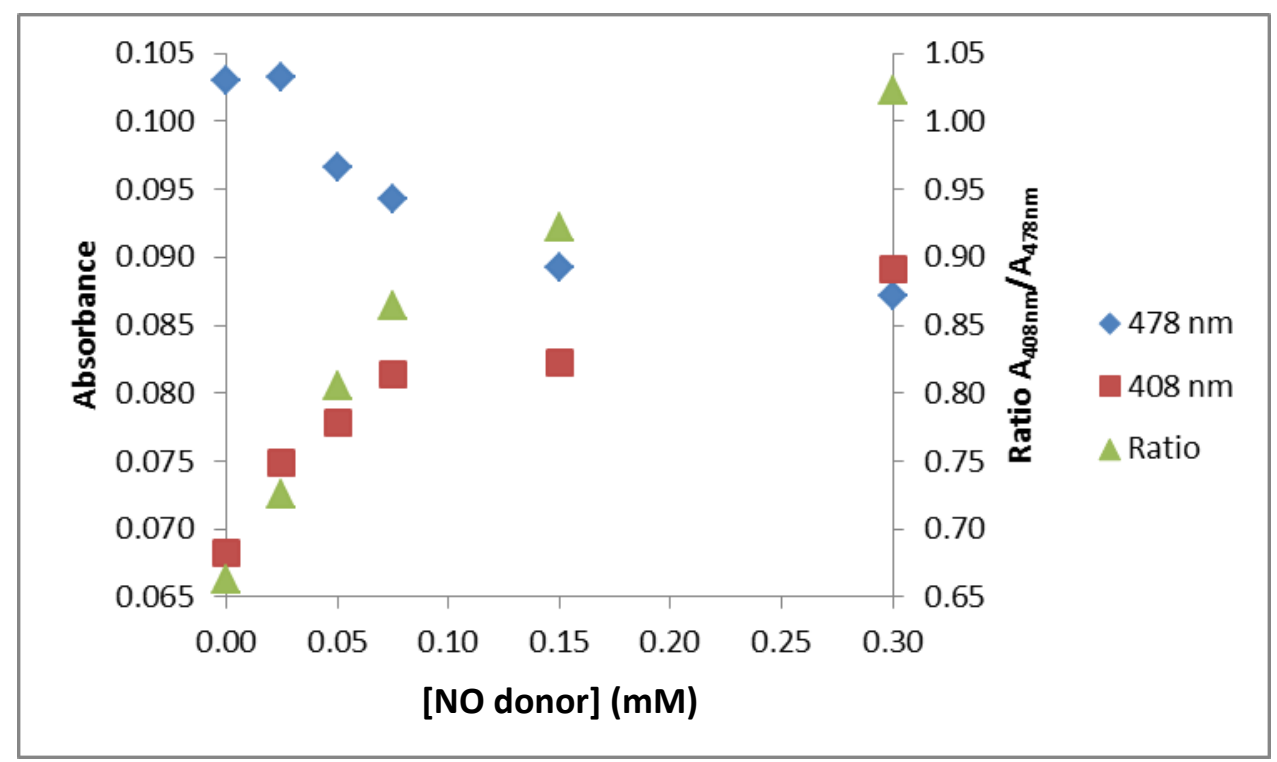

Figure 5.2 Relationship and ratio of the reaction of (Xy-DAD) $\mathrm{Re}(\mathrm{CO})_{2}(\mathrm{THF}) \mathrm{OSO}_{2} \mathrm{CF}_{3}$ with NO-donor, acetone/PBS (1:1)

In the case of Figure 5.3, which shows the spectrum UV - Visible spectrum of 8 in acetonitrile/acetone/buffer $2 / 48 / 50$ of the interaction of (XyDAD) $\mathrm{Re}\left(\mathrm{CH}_{3} \mathrm{CN}\right)(\mathrm{CO})_{2} \mathrm{OSO}_{2} \mathrm{CF}_{3}$ (9) with NO-donor, in the form of MAHMA NONOate in $0.04 \mathrm{M} \mathrm{NaOH}$, from $0-200 \mu \mathrm{M}$. It can be seen that the signal decreases at $496 \mathrm{~nm}$ and shifts hypsochromically. In the case of figure 5.3 Interaction of 9 with NOdonor, it is shown that the signal at $496 \mathrm{~nm}$ decreases consistently when the NO-donor concentration increases. Like the previous case, this color change can be observed by the naked eye. 


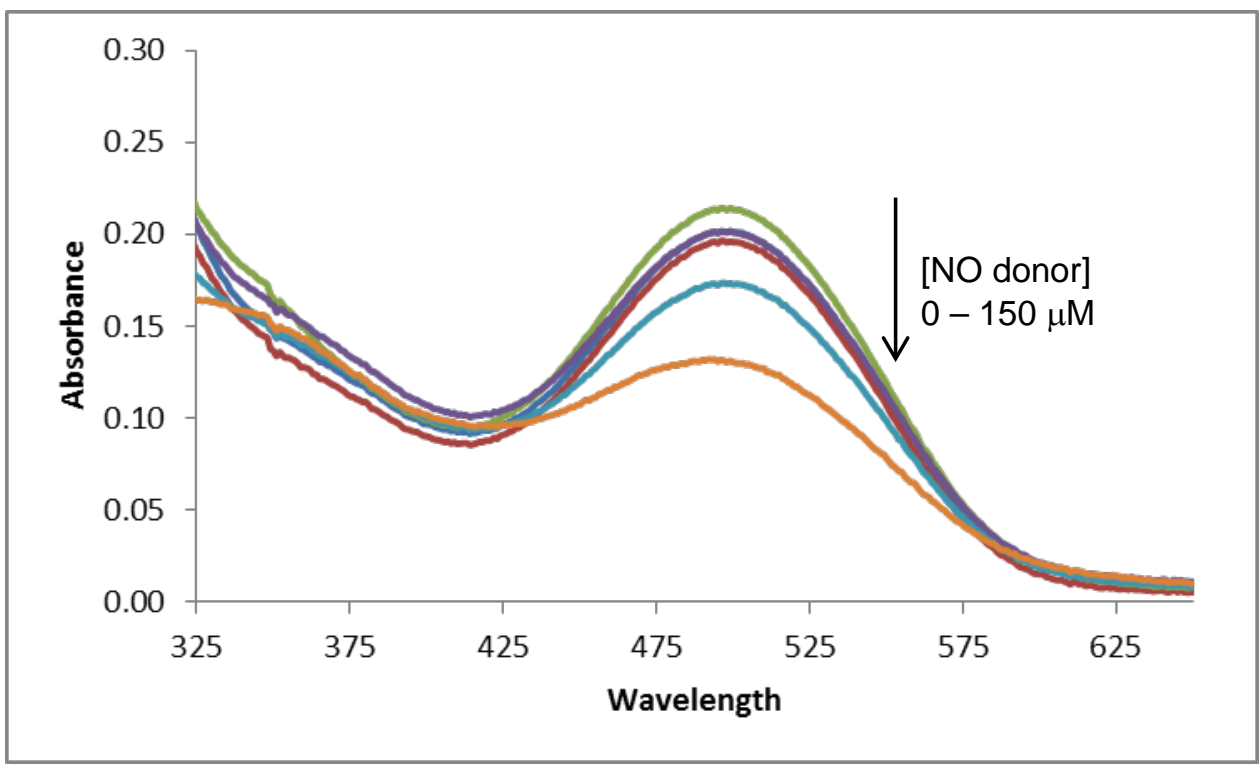

Figure 5.3 Interaction of (Xy-DAD) Re(CO) $)_{2}\left(\mathrm{CH}_{3} \mathrm{CN}\right) \mathrm{OSO}_{2} \mathrm{CF}_{3}$ with $\mathrm{NO}$-donor, Acetone/PBS (1:1)

The results of the interaction of NO-donor with (i-Pr$\mathrm{DAD}) \mathrm{Re}(\mathrm{CO})_{2}\left(\mathrm{CH}_{3} \mathrm{CN}\right) \mathrm{OSO}_{2} \mathrm{CF}_{3}$ and $(i-\mathrm{Pr}-\mathrm{DAD}) \mathrm{Re}(\mathrm{CO})_{2}(\mathrm{THF}) \mathrm{OSO}_{2} \mathrm{CF}_{3}$ were not clear because of the lower solubility of those complexes in mixed aqueous media. Even though the tendency was still observed, there was precipitation, which produced scattering and gave erratic absorbances.

\subsubsection{Reactivity of $\alpha$-diimine complexes with NaCN:}

In the case of Figure 5.4, which shows the spectrum UV - Visible spectrum of 8 in THF/acetone/buffer 2/48/50 of the interaction of (Xy-DAD)Re(THF)(CO) ${ }_{2} \mathrm{OSO}_{2} \mathrm{CF}_{3}$ (9) with $\mathrm{NaCN}$, from $0-100 \mu \mathrm{M}$. It can be seen that the signal decreases at $480 \mathrm{~nm}$ and shifts hypsochromically. In the case of figure 5.4 Interaction of $\mathbf{8}$ with $\mathrm{NaCN}$, it is shown that the signal at $480 \mathrm{~nm}$ increases consistently when the NO-donor concentration increases. Like the previous case, this color change can be observed by the naked eye. There is a little decrease of the signal at $344 \mathrm{~nm}$. 


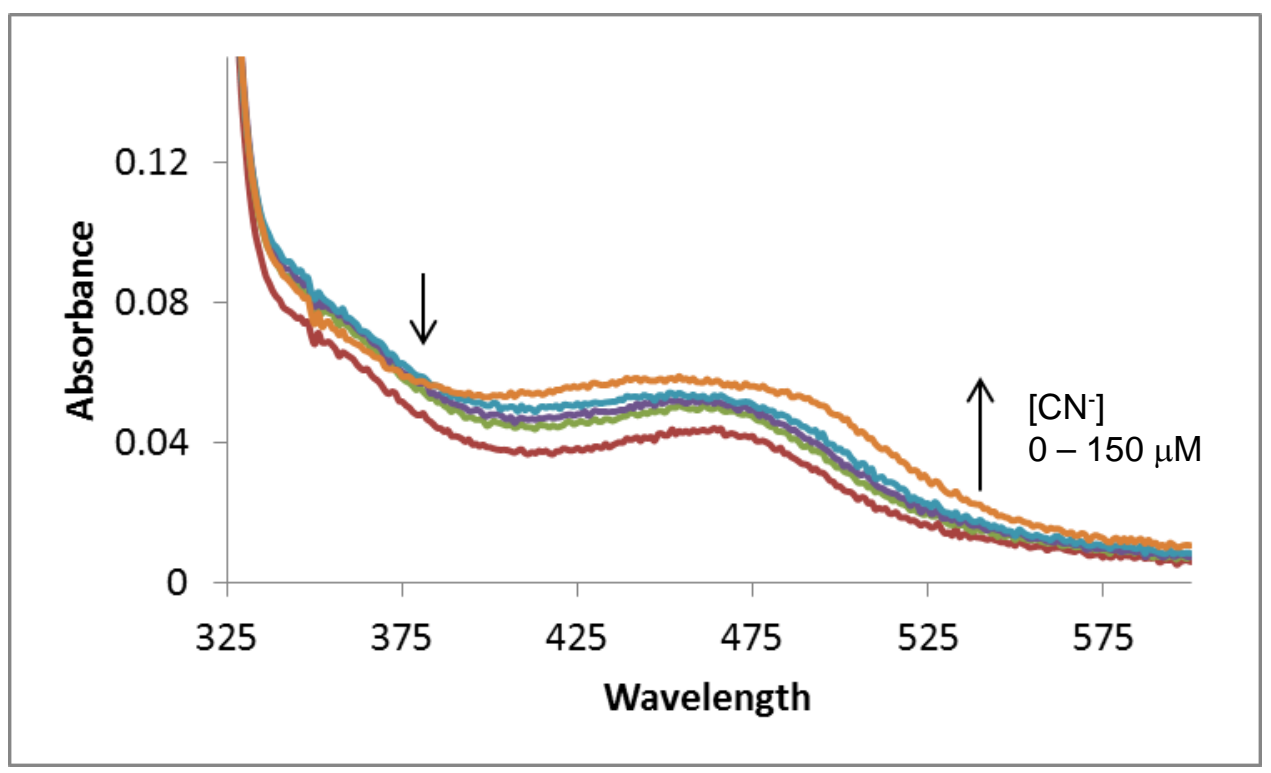

Figure 5.4 Interaction of (Xy-DAD) $\mathrm{Re}(\mathrm{CO})_{2}(\mathrm{THF}) \mathrm{OSO}_{2} \mathrm{CF}_{3}$ with $\mathrm{NaCN}$, acetone/PBS (1:1)

In the case of Figure 5.5, which shows the spectrum UV - Visible of the interaction of (Xy-DAD) $\mathrm{Re}\left(\mathrm{CH}_{3} \mathrm{CN}\right)(\mathrm{CO})_{2} \mathrm{OSO}_{2} \mathrm{CF}_{3}(\mathbf{9})$ with $\mathrm{NaCN}$ from $0-100 \mu \mathrm{M}$. It can be seen that the signal decreases at $500 \mathrm{~nm}$ and shifts hypsochromically, meanwhile the absorbance at $348 \mathrm{~nm}$ increases with an isosbestic point around $361 \mathrm{~nm}$. This color change can be observed by the naked eye. 


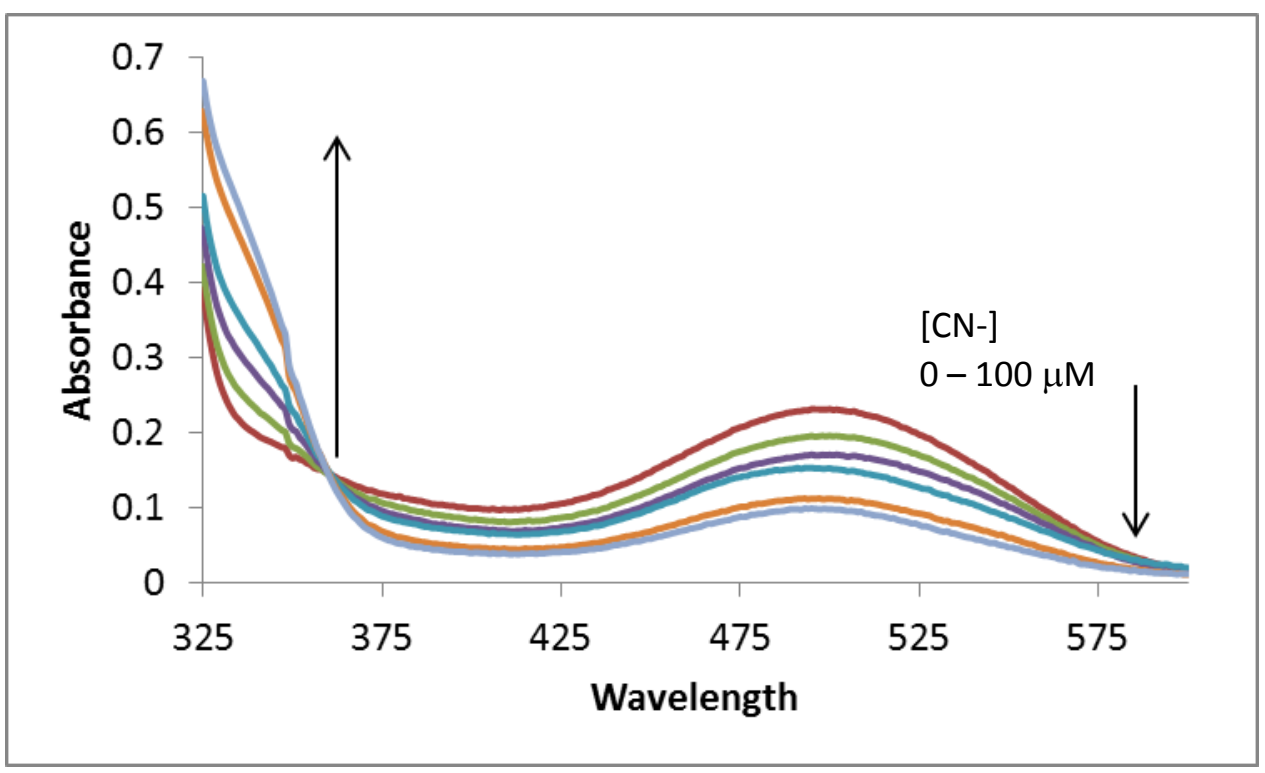

Figure 5.5 Interaction of $(\mathrm{Xy}-\mathrm{DAD}) \mathrm{Re}(\mathrm{CO})_{2}\left(\mathrm{CH}_{3} \mathrm{CN}\right) \mathrm{OSO}_{2} \mathrm{CF}_{3}$ with $\mathrm{NaCN}$, acetone/PBS (1:1)

The graph also shows the ratio of the absorbance at $500 \mathrm{~nm}$ vs the absorbance at $348 \mathrm{~nm}$. This plot shows a linear dependency at lower NO-donor concentrations. Figure 5.6 shows the plot of the local maxima and minima on the UV-Visible spectrum of 9 upon $\mathrm{CN}^{-}$gradual addition, in the form of $\mathrm{NaCN}(0-100 \mu \mathrm{M})$. The graph also shows the ratio of the absorbance at $348 \mathrm{~nm}$ vs the absorbance at $500 \mathrm{~nm}$. This plot shows a linear dependency at lower NO-donor concentrations. 


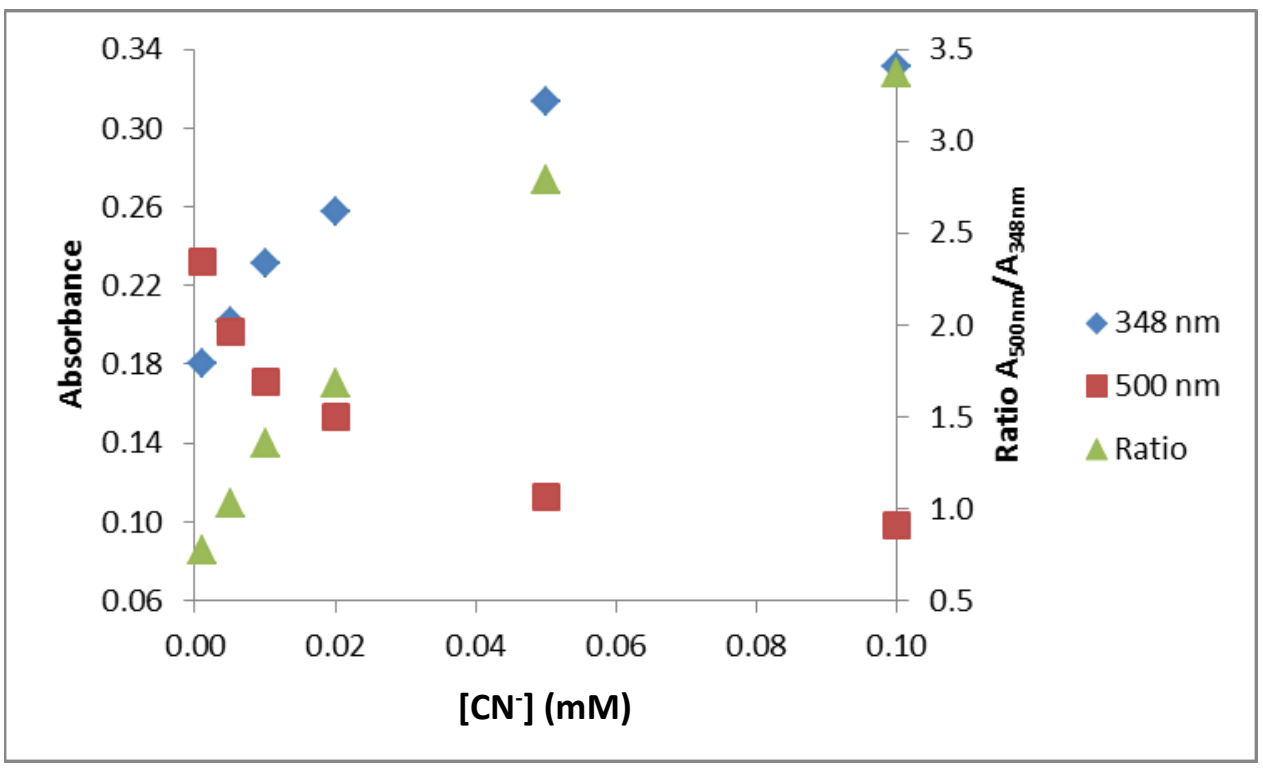

Figure 5.6 Relationship and ratio of the reaction of (Xy$\mathrm{DAD}) \mathrm{Re}(\mathrm{CO})_{2}\left(\mathrm{CH}_{3} \mathrm{CN}\right) \mathrm{OSO}_{2} \mathrm{CF}_{3}$ with $\mathrm{NaCN}$, acetone/PBS (1:1)

The results for the interaction of $\mathrm{NaCN}$ with (i-Pr$\mathrm{DAD}) \operatorname{Re}(\mathrm{CO})_{2}\left(\mathrm{CH}_{3} \mathrm{CN}\right) \mathrm{OSO}_{2} \mathrm{CF}_{3}$ and $(i-\mathrm{Pr}-\mathrm{DAD}) \mathrm{Re}(\mathrm{CO})_{2}(\mathrm{THF}) \mathrm{OSO}_{2} \mathrm{CF}_{3}$ were not clear because of the lower solubility of those complexes in mixed aqueous media. Even though the tendency was still observed, there was precipitation, which produced scattering and gave erratic absorbances.

\subsubsection{Comparison studies with other ions:}

A study was conducted in order to investigate the behavior of these dyes in the presence of competitive anions and/or oxidant species. Re(I) centers and DAD ligands are known by their redox properties. The selected anions and oxidant species were $\mathrm{NO}_{3}{ }^{-}$, $\mathrm{NO}_{2}^{-}, \mathrm{ClO}_{4}^{-}, \mathrm{ClO}^{-}, \mathrm{H}_{2} \mathrm{O}_{2}, \mathrm{CN}^{-}$and ${ }^{1} \mathrm{O}_{2}$ (this one was prepared in situ by reacting $\mathrm{H}_{2} \mathrm{O}_{2}$ with $\mathrm{ClO}^{-}$). Also, it was investigated whether the dyes still have activity towards NO after the reaction with these species. 
Figure 5.7 shows the UV - Vis maxima of (Xy-DAD) $\mathrm{Re}(\mathrm{THF})(\mathrm{CO})_{2} \mathrm{OSO}_{2} \mathrm{CF}_{3}$ (8) at $408 \mathrm{~nm}$, in the presence of strong oxidants the color (qualitative) and the absorbance at $500 \mathrm{~nm}$ (quantitative) decreases to almost zero. In the presence of weak oxidants the color was diluted, in the presence of $\mathrm{CN}^{-}$the solution turned yellow and the spectrum change considerably with a maximum of $348 \mathrm{~nm}$.

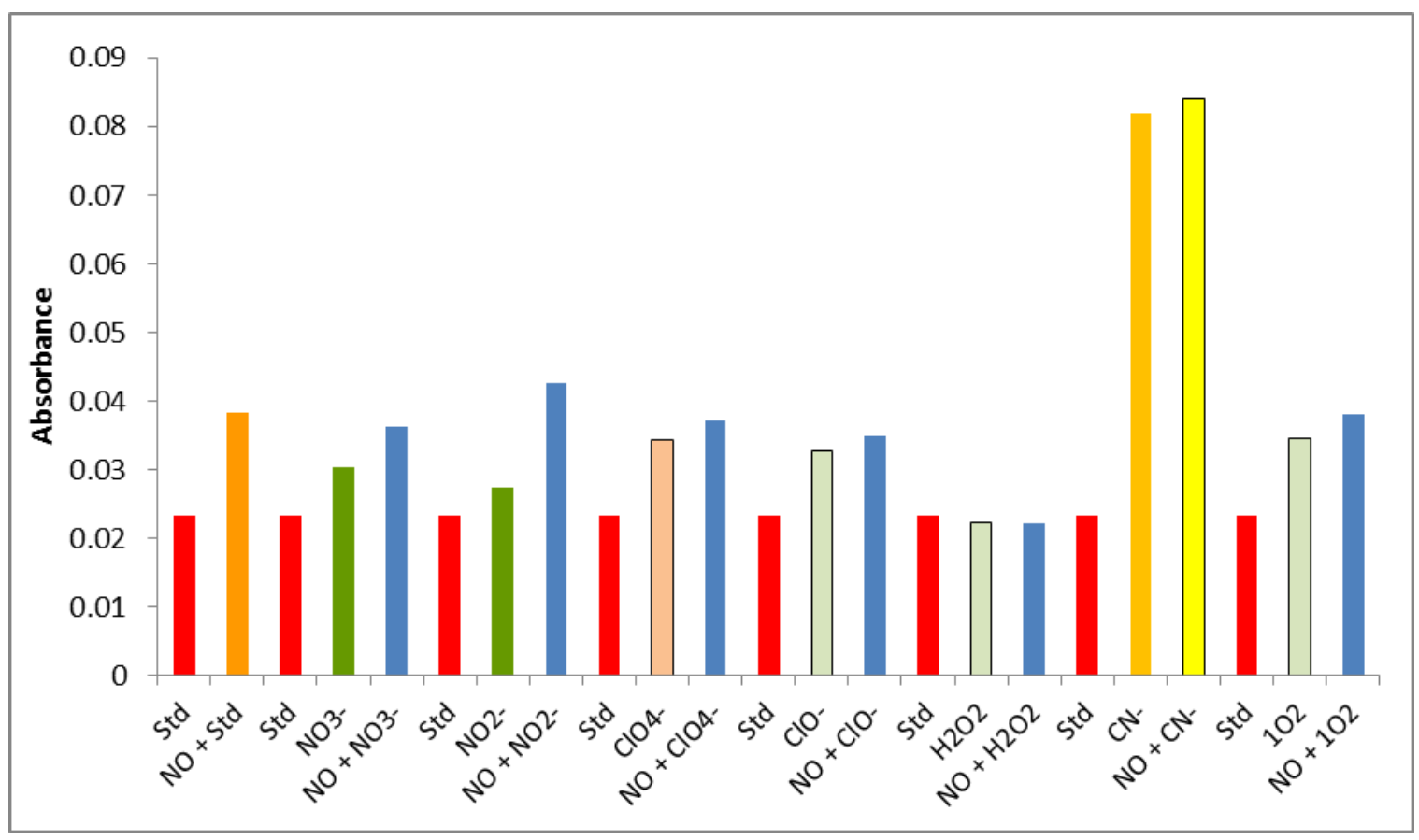

Figure 5.7 Comparison Studies among (Xy-DAD)Re(THF)(CO) ${ }_{2} \mathrm{OSO}_{2} \mathrm{CF}_{3}$ with NOdonor and Different Ions at $500 \mathrm{~nm}$

Figure 5.8 shows the UV - Vis maxima of (XyDAD) $\mathrm{Re}\left(\mathrm{CH}_{3} \mathrm{CN}\right)(\mathrm{CO})_{2} \mathrm{OSO}_{2} \mathrm{CF}_{3}(9)$ at $500 \mathrm{~nm}$, in the presence of strong oxidants the color (qualitative) and the absorbance at $500 \mathrm{~nm}$ (quantitative) decreases to almost zero. In the presence of weak oxidants the color was diluted, in the presence of $\mathrm{CN}^{-}$the solution turned yellow and the spectrum change considerably with a maximum of 348 $\mathrm{nm}$. In the case of $\mathrm{CN}^{-}$, there is not difference between the $\mathbf{8}$ and $\mathbf{9}$ spectra. There is possibly substitution of THF or $\mathrm{CH}_{3} \mathrm{CN}$ for $\mathrm{CN}^{-}$. 


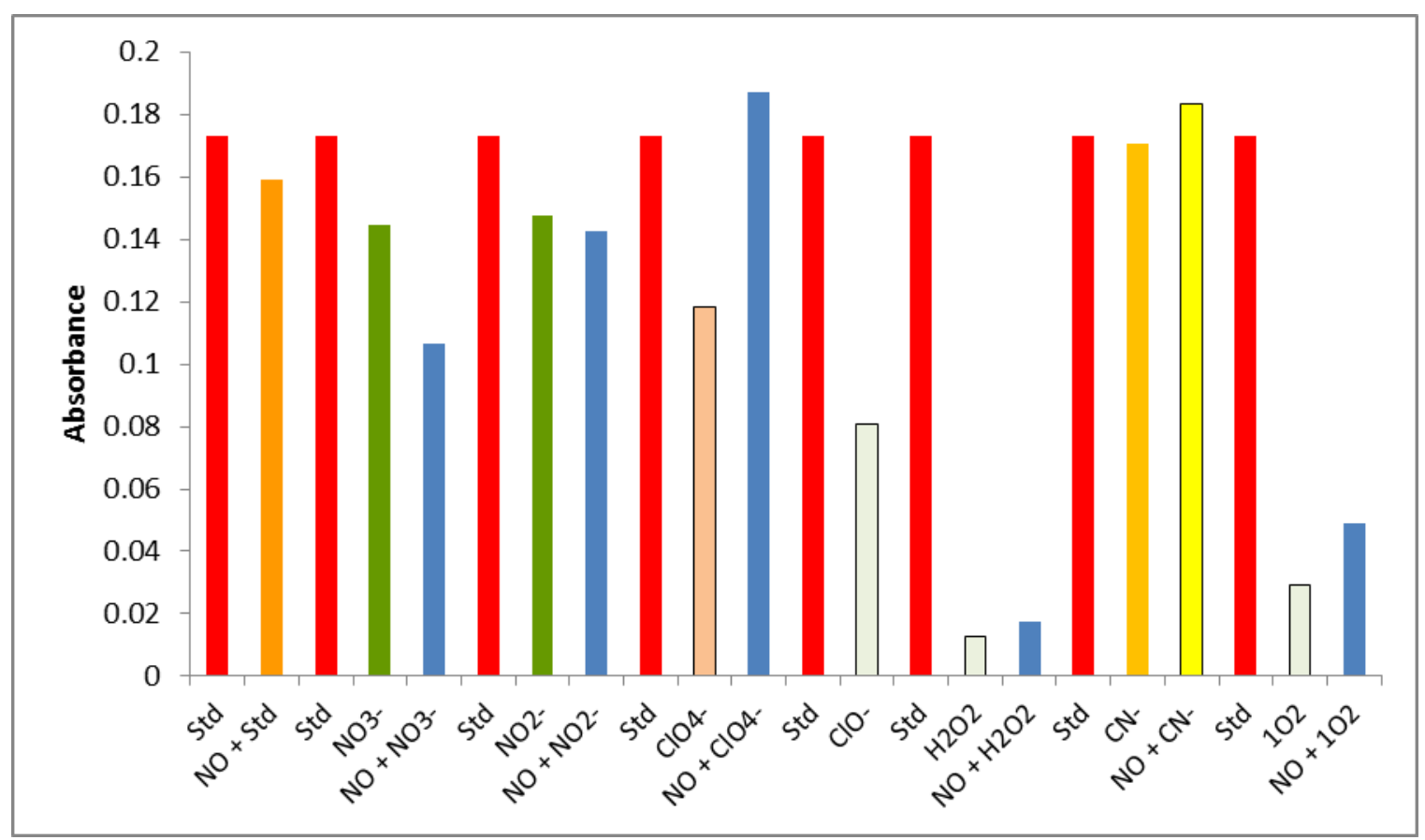

Figure 5.8 Comparison studies among (Xy-DAD) Re(CH3 $\mathrm{CN})(\mathrm{CO})_{2} \mathrm{OSO}_{2} \mathrm{CF}_{3}$ with NO-donor and Different Ions at $500 \mathrm{~nm}$

The results obtained by (Xy-DAD) $\mathrm{Re}\left(\mathrm{CH}_{3} \mathrm{CN}\right)(\mathrm{CO})_{2} \mathrm{OSO}_{2} \mathrm{CF}_{3}$ and (Xy-DAD)Re(THF)(CO) ${ }_{2} \mathrm{OSO}_{2} \mathrm{CF}_{3}$ in the presence of competitive ions can be summarized as follows: a) in the presence of $\mathrm{NO}_{2}{ }^{-}$, for both dyes, there were minor changes, and activity toward NO was not affected; b) in the presence of weak oxidants $\mathrm{NO}_{3}{ }^{-}$and $\mathrm{ClO}_{4}{ }^{-}$, the results were not conclusive; activity toward $\mathrm{NO}$ was partially lost because of the partial oxidation of $\operatorname{Re}(\mathrm{I})$ center; c) in the case of $\mathrm{CN}^{-}$, there was ligand substitution, and the reaction exhibited a change of color from red to yellow - $\mathrm{CN}^{-}$and NO are iso-electronics/iso-energetic and they compete for the same soft metal center; d) in the case of the strong oxidants $\mathrm{ClO}^{-}, \mathrm{H}_{2} \mathrm{O}_{2}$, and ${ }^{1} \mathrm{O}_{2}$, the color was lost completely, in addition to the activity toward NO, $\mathrm{d}^{6} \mathrm{Re}(\mathrm{I})$ possesses a ${ }^{3} \mathrm{MLCT}$ transition, which is responsible for generating the optical response (as describe in section 1.4.3.) toward NO. Oxidation, partial or total, of the metal center from $\operatorname{Re}(\mathrm{I})$ to $\operatorname{Re}(\mathrm{III})$ deactivates the 
organometallic dye because $\mathrm{d}^{4} \mathrm{Re}(\mathrm{III})$ does not possess ${ }^{3} \mathrm{MLCT}$ transition, which is required for the optical response.

\subsection{CONCLUSIONS}

Organometallic $\mathrm{d}^{6} \operatorname{Re}(\mathrm{I})$ complexes, with the DAD ligands, which can be used as NO and cyanide sensors. Re-DAD complexes are less soluble in mixed aqueous media than the phenanthroline analogs; they need twice the amount of acetone in the solvent

mixture. Xy-DAD complexes are more soluble than $i$-Pr-DAD ones. In the presence of oxidants, the Re complexes are deactivated by oxidation presumably because non- $\mathrm{d}^{6}$ Re centers do not possess the ${ }^{3}$ MLCT transition state needed for the optical response.

\subsection{REFERENCES}

1. Kavallieratos, K; Tsoukias, N. M.; Lozano-Lewis, L. I. "Organometallic dyes for NO detection and imaging". Issued Patent US 8,906,694 B2. Dec. 9, 2014.

2. Snyder, S. H.; Bredt, D. S. Sci Am. 1992, 266, 68-77.

3. (a) Tuteja, N.; Chandra, M.; Tuteja, R.; Misra, M. J.Biomed. Biotechnol., 2004, 4, 227-237; (b) Dredt, D. S. W.; Snyder, S. H. Annu. Rev. Biochem. 1994, 63, 175-195; (c) Wink, D. A.; Vodovotz, Y.; Laval, J.; Laval, F.; Dewhirst, M. W.; Mitchell, J. B. Carcinogenesis, 1998, 19, 711-721; (d) Calabrese, V.; Bates, T.E.; Stella, A. M. G. Neurochem. Res., 2000, 25, 1315-1341.

4. Kojima, H.; Nakatsubo, N.; Kikuchi, K.; Kawahara, S.; Kirino, Y.; Nagoshi, H.; Hirata, Y.; Nagano, T. Anal. Chem., 1998, 70, 2446-2453.

5. Hollis, T. K.; Odenkirk, W.; Robinson, N. P.; Whelan, J.; Bosnich, B. Tetrahedron, 1993, 49, 5415; (b) Odenkirk, W.; Rheingold, A. L.; Bosnich, B. J. Am. Chem. Soc., 1992, 114, 6392; (c) Failer, J. W.; Ma, Y.; Smart, C. J.; DiVerdi, M. J. J. Organomet. Chem. 1991, 420, 237; (d) Failer, J. W.; Ma, Y. J. Am. Chem. Soc. 1991, 113, 1579; (e) Honeychuck, R. V.; Bonnesen, P. V.; Farahi, J.; Hersh, W. H.; J. Org. Chem. 1987, 52, 5296; (f) Bonnesen, P. V.; Puckett, C. L.; Honeychuck, R. V.; Hersh, W. H. J. Am. Chem. Soc. 1989, 111, 6070.

6. (a)Veghini, D.; Nefedov, S. E.; Schmalle, H.; Berke, H. J. Organomet. Chem. 1996, 526, 117-134; (b) Machura, B. Coord. Chem. Rev. 2005, 249, 2277-2307 
7. Patra, S. C., Roy, A. S., Manivannan, V., Weyhermüller, T., Ghosh, P. Dalton Trans., 2014, 43, 13731.

8. Patra, S. C., Weyhermüller, T., Ghosh, P. Inorg. Chem., 2014, 53, 2427; (b) Roy, A. S., Tuononen, H. M., Rath, S. P., Ghosh, P. Inorg. Chem., 2007, 46, 5942.

9. Van Koten, G., Brieze, K. Advances in Organometallic Chemistry, 1982, 21, 152-234. 


\section{CONCLUSION}

As conclusion of this study, $\left(\mathrm{LN}_{2}\right) \mathrm{Re}(\mathrm{L})(\mathrm{CO})_{2} \mathrm{OTf}$ complexes $\left(\mathrm{LN}_{2}=\right.$ phen or DAD; $\quad \mathrm{L}=\mathrm{THF}, \mathrm{CH}_{3} \mathrm{CN}$, and $\mathrm{PPh}_{3}$ ) can be used as NO fluorescence sensors. The response is significantly stronger when the reaction with NO-donor is performed under anaerobic conditions indicating that NO is the real substrate instead of NO oxidation products. Controlling the $\mathrm{NaCl}$ concentration in the buffer allows converting these dyes into ratiometric response dyes. The spectroscopy data elucidate a mechanism involving the detachment of two labile ligands. The complex (phen) $\mathrm{Re}\left(\mathrm{PPh}_{3}\right)(\mathrm{CO})_{2} \mathrm{OTf}(\mathbf{1})$ has the most value as NO sensing fluorescent dye, because of it has the highest quantum yield of the Re-family group, and it is easy to manipulate because of it is isolable and it can be stored in powder form. This project can be extended in the future to other fluorescent ligands and Re complexes with higher quantum yields that can sense NO under physiological conditions. 


\section{APPENDIX 1. STUDY OF THE FLUORESCENT ORGANOMETALLIC COMPLEX (dppz)Re(L)(CO) $)_{2} \mathrm{OSO}_{2} \mathrm{CF}_{3}$ AS A NO SENSOR (L=THF, $\mathrm{CH}_{3} \mathrm{CN}$, and $\left.\mathrm{PPh}_{3}\right)$.}

\section{A1.1. ABSTRACT}

Dipyrido[3,2-a:2',3'-c]phenazine (dppz) is used as fluorophore for DNA intercalator and fluorescent dye. (dppz)Re(THF)(CO) ${ }_{2} \mathrm{OSO}_{2} \mathrm{CF}_{3} \quad$ (A2), (dppz) $\mathrm{Re}\left(\mathrm{PPh}_{3}\right)(\mathrm{CO})_{2} \mathrm{OSO}_{2} \mathrm{CF}_{3}(\mathrm{~A} 3)$, and (dppz) $\mathrm{Re}\left(\mathrm{PPh}_{3}\right)(\mathrm{CO})_{2} \mathrm{OSO}_{2} \mathrm{CF}_{3}$ (A4), which can be obtained photochemically from (dppz) $\mathrm{Re}(\mathrm{CO})_{3} \mathrm{OSO}_{2} \mathrm{CF}_{3}$ (A1) shows fluorescence quenching response upon NO addition, which was added in the form of NOdonor Z-1-N-mthyl-N-[6-(N-methylammoniohexyl)amino]diazen-1-ium-1,2-diolate (MAHMA-NONOate). Addition of $10-150 \mu \mathrm{M}$ of NO-donor into a $25 \mu \mathrm{M}$ solution of (dppz) $\mathrm{Re}\left(\mathrm{CH}_{3} \mathrm{CN}\right)(\mathrm{CO})_{2} \mathrm{OSO}_{2} \mathrm{CF}_{3}$ or $(\mathrm{dppz}) \mathrm{Re}\left(\mathrm{CH}_{3} \mathrm{CN}\right)(\mathrm{CO})_{2} \mathrm{OSO}_{2} \mathrm{CF}_{3}$ in $25 \% \mathrm{v} / \mathrm{v}$ acetone/phosphate saline (PBS) buffer gave fluorescence quenching at $458 \mathrm{~nm}$ and 586nm, upon excitation at $360 \mathrm{~nm}$ and $380 \mathrm{~nm}$. The ${ }^{1} \mathrm{H}-\mathrm{NMR}$ and ${ }^{19} \mathrm{~F}-\mathrm{NMR}$ spectra after reaction with $\mathrm{NO}$ showed characteristic resonances for non-coordinated triflate and the $\mathrm{L}$ ligand $\left(\mathrm{L}=\mathrm{THF}, \mathrm{PPh}_{3}\right.$ or $\left.\mathrm{CH}_{3} \mathrm{CN}\right)$, suggesting that the organometallic product formed upon NO releases both these ligands upon reaction with NO. This study expands our newly demonstrated approach for the design of fluorescent NO sensors based on NO coordination to Re; ${ }^{1} \mathbf{A 1}$ is an isolable complex, which is stable at room temperature and therefore can be used as starting material for NO sensing organometallic dyes.

\section{A1.2. INTRODUCTION}

Fluorescent materials are attracting considerable interest due to their potential sensory, biochemical, medical, and photoelectronic applications, in addition to other 
possible uses. ${ }^{2}$ One type of fluorescent material that have been attracting attention are Dipyrido[3,2-a:2',3'-c]phenazine $\mathrm{Ru}(\mathrm{II})$ complexes. They have been used as "light switches” when they bind DNA. ${ }^{3}$ Through NMR, it was proved that Ru(phen) $2(\mathrm{dppz})^{+2}$ intercalated double stranded DNA employing the dppz moiety. Ru(dppz) complexes were also used as electrochemical indicators for DNA double-helix's detection. ${ }^{4}$

In many metal polypyridyl complexes, the most important state is the metal-toligand charge-transfer (MLCT) state, which is important to defining photophysical properties. ${ }^{5}$ The Barton et al. study proved that dppz become emissive when the nitrogens are shielded from water; otherwise, these complexes are non-emissive. ${ }^{6}$

Many photophysical and theoretical studies have suggested that $\operatorname{Re}(\mathrm{I})-$ and $\mathrm{Ru}(\mathrm{II})$-dppz complexes exhibit two low-energy triplet metal to ligand charge-transfer (MLCT) states and one triplet ligand-centered state. ${ }^{7}$ The ${ }^{3}$ MLCT (phen) state is emissive because of environment stabilization, while the ${ }^{3}$ MLCT (phz) state is a dark state. ${ }^{8}$

dppz complexes are weakly emissive in $\mathrm{CH}_{2} \mathrm{Cl}_{2}$. For most complexes, their emission is short, decaying in less than $10 \mathrm{~ns}$ after the laser pulse. ${ }^{9}$ It was found that $\left[\operatorname{Re}(\mathrm{CO})_{3}(\mathrm{dppz}) \mathrm{Cl}\right]$ complexes possess MLCT states based on the phen portion of the dppz ligands, which does not occur in $\left[\operatorname{Re}(\mathrm{CO})_{3}(\mathrm{dppz})(\mathrm{py})\right] \mathrm{PF}_{6}$, which is $\mathrm{L}^{+1}$, in which the phenazine is responsible of the transition. Many of the molecular orbitals in these compounds show extensive delocalization across the dppz ligand, in particular for these cationic species, which have $\mathrm{L}^{+1}$ extended over the whole dppz system (Figure A1.1) destabilizing the orbital. 

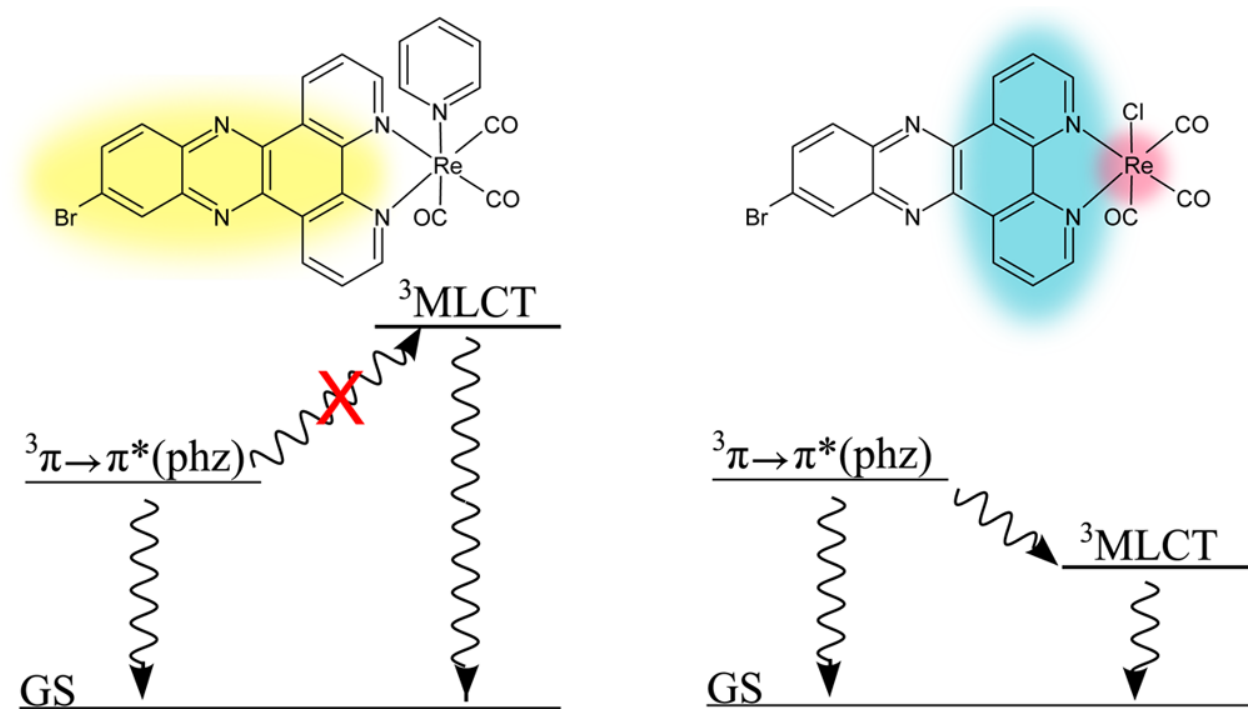

Figure A1.1 Energy diagram for (a) $\left[(\mathrm{dppz}) \mathrm{Re}(\mathrm{CO})_{3}(\mathrm{py})\right] \mathrm{PF}_{6}$ and (b) $(\mathrm{dppz}) \operatorname{Re}(\mathrm{CO})_{3} \mathrm{Cl}$

\section{A1.3. EXPERIMENTAL SECTION}

\section{A1.3.1. Materials and Methods}

$(\mathrm{dppz}) \mathrm{Re}(\mathrm{CO}){ }_{3} \mathrm{Cl}^{10}$ was prepared with a modification of the previously reported procedure for (phen)Re(CO) ${ }_{3} \mathrm{Cl}^{11}$ All other materials (purchased from Aldrich Chemical Co., ACROS organics, Cayman Chemicals, or Alfa Aesar) were standard reagent grade and were used without further purification, unless otherwise noted. PBS buffer (0.002 M in total phosphate, $\mathrm{pH}=7.2,[\mathrm{NaCl}]=0.04 \mathrm{M}$ ) was prepared from $\mathrm{K}_{2} \mathrm{HPO}_{4}, \mathrm{KH}_{2} \mathrm{PO}_{4}$, and $\mathrm{NaCl}$ (all from Fisher Scientific). Filtered water $\left(18 \mathrm{M} \Omega \cdot \mathrm{cm}^{-1}\right)$ produced from a Millipore ${ }^{\circledR}$ nanopure diamond lab water system (Barnstead Thermolyne Corporation, Dubuque, IA) was used for preparation of the buffers and experiments with NO. Deuterated solvents were purchased from Cambridge Isotope Laboratories Ltd. ${ }^{1} \mathrm{H}$ and ${ }^{13} \mathrm{C}$ NMR spectra were recorded on a 400 Bruker NMR spectrometer and were referenced, using the residual solvent resonances. All chemical shifts, $\delta$, are reported in 
ppm. FT-IR spectra were recorded with a Perkin Elmer Spectrum 100 FT-IR Spectrometer.

\section{A1.3.2. Synthesis of $(\mathrm{dppz}) \operatorname{Re}(\mathrm{CO}){ }_{3} \mathrm{OSO}_{2} \mathrm{CF}_{3}$ :}

The complex (dppz) $\mathrm{Re}(\mathrm{CO})_{3} \mathrm{OSO}_{2} \mathrm{CF}_{3}$ was prepared by a modification of the previously reported procedure for (phen) $\mathrm{Re}(\mathrm{CO})_{3} \mathrm{OSO}_{2} \mathrm{CF}_{3} .{ }^{12} \quad 150.0 \mathrm{mg}$ of (dppz)Re(CO) ${ }_{3} \mathrm{Cl}(255 \mu \mathrm{mol})$ and $10 \mathrm{~mL}$ of dichloromethane (final concentration: 25.5 $\mathrm{mM}$ ) were placed in a $50 \mathrm{~mL}$ round bottom flask under $\mathrm{N}_{2}$ flow. $\quad 250 \mu \mathrm{L}$ of $\mathrm{HOSO}_{2} \mathrm{CF}_{3}$ were added dropwise in a 30 minute period, after the solution was left stirring for $2 \mathrm{~h}$. The color changed during reflux from orange to red. $250 \mu \mathrm{L}$ of ethanol were added and the volatiles were evaporated to $1.0 \mathrm{~mL}$. The product was precipitated at room temperature by adding $4.0 \mathrm{~mL}$ of diethyl ether. The bright orange powder product was filtered and was dried in vacuo at $40{ }^{\circ} \mathrm{C} .168 .1 \mathrm{mg},(93 \%) .{ }^{1} \mathrm{H}-\mathrm{NMR}\left(\mathrm{CDCl}_{3}\right)$ : 8.09 (dd, $\left.2 \mathrm{H},{ }^{3} \mathrm{~J}_{\mathrm{H}-\mathrm{H}}=6.6 \mathrm{~Hz},{ }^{3} \mathrm{~J}_{\mathrm{H}-\mathrm{H}}=3.4 \mathrm{~Hz}\right), 8.10\left(\mathrm{dd}, 2 \mathrm{H},{ }^{3} \mathrm{~J}_{\mathrm{H}-\mathrm{H}}=8.2 \mathrm{~Hz},{ }^{3} \mathrm{~J}_{\mathrm{H}-\mathrm{H}}=5.2 \mathrm{~Hz}\right), 8.48$ $\left(\mathrm{dd}, 2 \mathrm{H},{ }^{3} \mathrm{~J}_{\mathrm{H}-\mathrm{H}}=6.6 \mathrm{~Hz},{ }^{3} \mathrm{~J}_{\mathrm{H}-\mathrm{H}}=3.4 \mathrm{~Hz}\right), 9.50\left(\mathrm{dd}, 2 \mathrm{H},{ }^{3} \mathrm{~J}_{\mathrm{H}-\mathrm{H}}=5.2 \mathrm{~Hz},{ }^{3} \mathrm{~J}_{\mathrm{H}-\mathrm{H}}=1.4 \mathrm{~Hz}\right)$, $9.96\left(\mathrm{dd}, 2 \mathrm{H},{ }^{3} \mathrm{~J}_{\mathrm{H}-\mathrm{H}}=8.2 \mathrm{~Hz},{ }^{3} \mathrm{~J}_{\mathrm{H}-\mathrm{H}}=1.4 \mathrm{~Hz}\right) .{ }^{19} \mathrm{~F}-\left\{{ }^{1} \mathrm{H}\right\}-\mathrm{NMR}\left(\mathrm{CDCl}_{3}\right):-78.1 \mathrm{ppm} . \quad \mathrm{FT}-$ IR (cm $\left.{ }^{-1}\right): 2015$ (vco, s), 1875 (vco, s), 1629, 1604, 1584, 1520, 1427, 1417, 1350, 1321, $1277,1255,1223,1145,1109,1058,1030,908,848,808,780,723,638$. UV-Vis (in ethanol): $\lambda_{\max }=360 \mathrm{~nm}$ - br $\left(\varepsilon=1840 \mathrm{~mol}^{-1} \mathrm{~cm}^{-1}\right), 380 \mathrm{~nm}-\left(\varepsilon=1840 \mathrm{~mol}^{-1} \mathrm{~cm}^{-1}\right)$.

\section{A1.3.3. Photochemical substitution:}

All the solutions were irradiated with a Rayonet photochemical reactor (Southern New England Ultraviolet, model RPR-100), equipped with a cooling fan and 10 phosphor coated low-pressure mercury lamps $\left(\lambda_{\max }=300 \mathrm{~nm}\right.$, light intensity $=5.2 \pm 0.1 \times 10^{6}$ 
photon/sec/ $\mathrm{cm}^{3}$ ). After $75 \mathrm{~min}$. of irradiation, the colors of the solutions changed to darker colors.

\section{A1.3.3.1. Synthesis of (dppz)Re(THF)(CO) ${ }_{2} \mathrm{OSO}_{2} \mathrm{CF}_{3}(\mathrm{~A} 2)$ :}

(dppz)Re(CO) ${ }_{3} \mathrm{OSO}_{2} \mathrm{CF}_{3}$ (A1) (1 mg, $\left.1.46 \mu \mathrm{mol}\right) 1.00 \mathrm{~mL}$ of anhydrous THF (final concentration $1.46 \mathrm{mM}$ ) were placed in a NMR tube. The solution was irradiated for $75 \mathrm{~min}$. at room temperature. This solution was used without further treatment. ${ }^{1} \mathrm{H}$ NMR $\left(\mathrm{CDCl}_{3}\right): 7.98\left(\mathrm{dd}, 2 \mathrm{H},{ }^{3} \mathrm{~J}_{\mathrm{H}-\mathrm{H}}=6.6 \mathrm{~Hz},{ }^{3} \mathrm{~J}_{\mathrm{H}-\mathrm{H}}=2.8 \mathrm{~Hz}\right), 8.12\left(\mathrm{dd}, 2 \mathrm{H},{ }^{3} \mathrm{~J}_{\mathrm{H}-\mathrm{H}}=8.2\right.$ $\left.\mathrm{Hz},{ }^{3} \mathrm{~J}_{\mathrm{H}-\mathrm{H}}=5.2 \mathrm{~Hz}\right), 8.48\left(\mathrm{dd}, 2 \mathrm{H},{ }^{3} \mathrm{~J}_{\mathrm{H}-\mathrm{H}}=6.6 \mathrm{~Hz},{ }^{3} \mathrm{~J}_{\mathrm{H}-\mathrm{H}}=3.4 \mathrm{~Hz}\right), 9.44\left(\mathrm{dd}, 2 \mathrm{H},{ }^{3} \mathrm{~J}_{\mathrm{H}-\mathrm{H}}=\right.$ $5.2 \mathrm{~Hz},{ }^{3} \mathrm{~J}_{\mathrm{H}-\mathrm{H}}=1.4 \mathrm{~Hz}$ ), $9.87\left(\mathrm{dd}, 2 \mathrm{H},{ }^{3} \mathrm{~J}_{\mathrm{H}-\mathrm{H}}=8.2 \mathrm{~Hz},{ }^{3} \mathrm{~J}_{\mathrm{H}-\mathrm{H}}=1.4 \mathrm{~Hz}\right) .{ }^{19} \mathrm{~F}-\left\{{ }^{1} \mathrm{H}\right\}-\mathrm{NMR}$ (CDCl $)$ : -78.4 ppm. FT-IR ( $\left.\mathrm{cm}^{-1}\right): 2032$ (vco, s), 1915 (vco, s), 1640, 1460, 1442, 1423, 1344, 1320, 1282, 1241, 1177, 1115, 1064, 1030, 908, 848, 808, 780, 723, 638.

\section{A1.3.3.2. Synthesis of $(\mathrm{dppz}) \mathrm{Re}\left(\mathrm{CH}_{3} \mathrm{CN}\right)(\mathrm{CO})_{2} \mathrm{OSO}_{2} \mathrm{CF}_{3}(\mathrm{~A} 3)$ :}

(dppz)Re(CO) ${ }_{3} \mathrm{OSO}_{2} \mathrm{CF}_{3}(\mathbf{A 1})(1 \mathrm{mg}, 1.46 \mu \mathrm{mol}) 1.00 \mathrm{~mL}$ of acetonitrile (final concentration $1.46 \mathrm{mM}$ ) were placed in a NMR tube. The solution was irradiated for 75 minutes at room temperature. This solution was used without further treatment. ${ }^{1} \mathrm{H}-$ NMR (CDCl $): 2.24(\mathrm{~s}, 3 \mathrm{H}), 8.04\left(\mathrm{~d}, 2 \mathrm{H},{ }^{3} \mathrm{~J}_{\mathrm{H}-\mathrm{H}}=6.6 \mathrm{~Hz},{ }^{3} \mathrm{~J}_{\mathrm{H}-\mathrm{H}}=3.4 \mathrm{~Hz}\right), 8.13(\mathrm{dd}$, $\left.2 \mathrm{H},{ }^{3} \mathrm{~J}_{\mathrm{H}-\mathrm{H}}=8.2 \mathrm{~Hz},{ }^{3} \mathrm{~J}_{\mathrm{H}-\mathrm{H}}=5.2 \mathrm{~Hz}\right), 8.46\left(\mathrm{dd}, 2 \mathrm{H},{ }^{3} \mathrm{~J}_{\mathrm{H}-\mathrm{H}}=6.6 \mathrm{~Hz},{ }^{3} \mathrm{~J}_{\mathrm{H}-\mathrm{H}}=3.4 \mathrm{~Hz}\right), 9.38(\mathrm{~d}$, $\left.2 \mathrm{H},{ }^{3} \mathrm{~J}_{\mathrm{H}-\mathrm{H}}=4.4 \mathrm{~Hz}\right), 10.01\left(\mathrm{dd}, 2 \mathrm{H},{ }^{3} \mathrm{~J}_{\mathrm{H}-\mathrm{H}}=7.3 \mathrm{~Hz}\right) .{ }^{19} \mathrm{~F}-\left\{{ }^{1} \mathrm{H}\right\}-\mathrm{NMR}\left(\mathrm{CDCl}_{3}\right):-78.3 \mathrm{ppm}$. FT-IR ( $\left.\mathrm{cm}^{-1}\right): 2322\left(v_{\mathrm{CN}}, \mathrm{w}\right), 2042\left(v_{\mathrm{co}}, \mathrm{vs}\right), 1953$ ( $\left.v_{\mathrm{CO}}, \mathrm{vs}\right), 1941$ ( $\left.v_{\mathrm{CO}}, \mathrm{vs}\right), 1924$ ( $v_{\mathrm{CO}}$ vs), 1642, 1496, 1422, 1358, 1283, 1252, 1226, 1163, 1031, 820, 775, 731, 639.

\section{A1.3.3.3. Synthesis of $(\mathrm{dppz}) \mathrm{Re}\left(\mathrm{PPh}_{3}\right)(\mathrm{CO})_{2} \mathrm{OSO}_{2} \mathrm{CF}_{3}(\mathrm{~A} 4)$ :}

(dppz)Re(CO) ${ }_{3} \mathrm{OSO}_{2} \mathrm{CF}_{3}(\mathbf{A 1})(1 \mathrm{mg}, 1.46 \mu \mathrm{mol}) 1.00 \mathrm{~mL}$ of acetonitrile (final concentration $1.46 \mathrm{mM}$ ) were placed in a NMR tube. The solution was irradiated for 75 
minutes at room temperature. This solution was used without further treatment. ${ }^{1} \mathrm{H}-$ NMR (CDCl3): ): 6.96 (t, 3H, $\left.{ }^{3} \mathrm{~J}_{\mathrm{H}-\mathrm{H}}=7.0 \mathrm{~Hz}\right), 7.39\left(\mathrm{dd}, 6 \mathrm{H},{ }^{3} \mathrm{~J}_{\mathrm{H}-\mathrm{H}}=7.6 \mathrm{~Hz},{ }^{3} \mathrm{~J}_{\mathrm{H}-\mathrm{H}}=2.0 \mathrm{~Hz}\right), 7.62$ $\left(\mathrm{dd}, 6 \mathrm{H},{ }^{3} \mathrm{~J}_{\mathrm{H}-\mathrm{H}}=12.5 \mathrm{~Hz},{ }^{3} \mathrm{~J}_{\mathrm{H}-\mathrm{H}}=7.2 \mathrm{~Hz}\right), 8.06\left(\mathrm{~d}, 2 \mathrm{H},{ }^{3} \mathrm{~J}_{\mathrm{H}-\mathrm{H}}=6.6 \mathrm{~Hz},{ }^{3} \mathrm{~J}_{\mathrm{H}-\mathrm{H}}=3.4 \mathrm{~Hz}\right), 8.09$ $\left(\mathrm{dd}, 2 \mathrm{H},{ }^{3} \mathrm{~J}_{\mathrm{H}-\mathrm{H}}=8.3 \mathrm{~Hz},{ }^{3} \mathrm{~J}_{\mathrm{H}-\mathrm{H}}=5.2 \mathrm{~Hz}\right), 8.49\left(\mathrm{dd}, 2 \mathrm{H},{ }^{3} \mathrm{~J}_{\mathrm{H}-\mathrm{H}}=6.6 \mathrm{~Hz},{ }^{3} \mathrm{~J}_{\mathrm{H}-\mathrm{H}}=3.4 \mathrm{~Hz}\right)$,

$9.56\left(\mathrm{dd}, 2 \mathrm{H},{ }^{3} \mathrm{~J}_{\mathrm{H}-\mathrm{H}}=5.2 \mathrm{~Hz},{ }^{3} \mathrm{~J}_{\mathrm{H}-\mathrm{H}}=1.8 \mathrm{~Hz}\right), 9.93\left(\mathrm{dd}, 2 \mathrm{H},{ }^{3} \mathrm{~J}_{\mathrm{H}-\mathrm{H}}=8.3 \mathrm{~Hz},{ }^{3} \mathrm{~J}_{\mathrm{H}-\mathrm{H}}=1.8\right.$ Hz). ${ }^{19} \mathrm{~F}-\left\{{ }^{1} \mathrm{H}\right\}-\mathrm{NMR}\left(\mathrm{CDCl}_{3}\right):-78.4$ ppm FT-IR $\left(\mathrm{cm}^{-1}\right): 2032$ (vco, s), 1932 (vco, s), 1914 (vco, s), 1648, 1460, 1439, 1366, 1260, 1178, 1120, 1052 (s-br), 897, 803, 780, 723, 696.

\section{A1.3.4. Fluorescence experiments with NO-donor}

In a typical experiment, a $100 \mu \mathrm{M}$ solution of (dppz) $\mathrm{Re}(\mathrm{THF})(\mathrm{CO})_{2} \mathrm{OSO}_{2} \mathrm{CF}_{3}$ complex was prepared from $1.67 \mathrm{mM}$ stock solution of (dppz)Re(THF)(CO) ${ }_{2} \mathrm{OSO}_{2} \mathrm{CF}_{3}$ in THF and acetone was added up to $1.000 \mathrm{~mL}$ of total solution of Re complex. $2.75 \mathrm{~mL}$ to $3.00 \mathrm{~mL}$ of aqueous PBS buffer ( $\mathrm{pH}=7.2$ ) was added. Then 0 to $250 \mu \mathrm{L}$ of $4 \mathrm{mM}$ stock solution of MAHMA-NONOate NO-donor, in $0.04 \mathrm{M} \mathrm{NaOH}$, were added in order to obtain $75 \% \mathrm{v} / \mathrm{v}$ aqueous/organic solution and a final NO-donor concentration from 0 to $200 \mu \mathrm{M}$ range, while the concentration of Re complex remained constant at $25 \mu \mathrm{M}$. No more than 10 minutes elapsed from preparation of the organic phase to the addition of the NO-donor. The solution was mixed thoroughly in a vortex and sonicated for 90 minutes at room temperature. The fluorescence of all solutions was measured with a $\lambda_{\text {exc }}=360$ $\mathrm{nm}$ and $380 \mathrm{~nm}$. The emission spectrum was recorded from $400 \mathrm{~nm}$ to $700 \mathrm{~nm}$.

\section{A1.3.5. UV-Visible spectroscopy experiments with NO-donor}


The (dppz) $\mathrm{Re}(\mathrm{THF})(\mathrm{CO})_{2} \mathrm{OSO}_{2} \mathrm{CF}_{3}$ (A2), (dppz) $\mathrm{Re}(\mathrm{THF})(\mathrm{CO})_{2} \mathrm{OSO}_{2} \mathrm{CF}_{3}$ (A3), and (dppz)Re(THF)(CO) ${ }_{2} \mathrm{OSO}_{2} \mathrm{CF}_{3}$ (A4) samples were prepared in the same way that in A1.3.4. The UV-Visible spectra of all solutions were recorded from $300 \mathrm{~nm}$ to $600 \mathrm{~nm}$.

\section{A1.3.6. $\quad{ }^{1} \mathrm{H}$-NMR and ${ }^{19} \mathrm{~F}-\mathrm{NMR}$ Studies of dppz complexes}

The performed procedure was similar to sections 2.3.5 and 3.3.4; the stock solutions of A2, A3 and A4 after reaction with NO-donor were extracted with dichloromethane. The organic fractions were dried with $\mathrm{Na}_{2} \mathrm{SO}_{4}$ and the volatiles were evaporated. The residue was redissolved with chloroform-d and NMR was performed.

\section{A1.4. RESULTS AND DISCUSSION}

\section{A1.4.1. Synthesis of (dppz) $\operatorname{Re}(\mathrm{L})(\mathrm{CO})_{2} \mathrm{OSO}_{2} \mathrm{CF}_{3}$}

Complexes A2, A3 and A4 were synthesized by photochemical substitution of CO from (dppz) $\mathrm{Re}(\mathrm{CO})_{3} \mathrm{SO}_{3} \mathrm{CF}_{3}(\mathbf{A 1})$. The products were characterized by ${ }^{1} \mathrm{H}$ and FT-IR. 


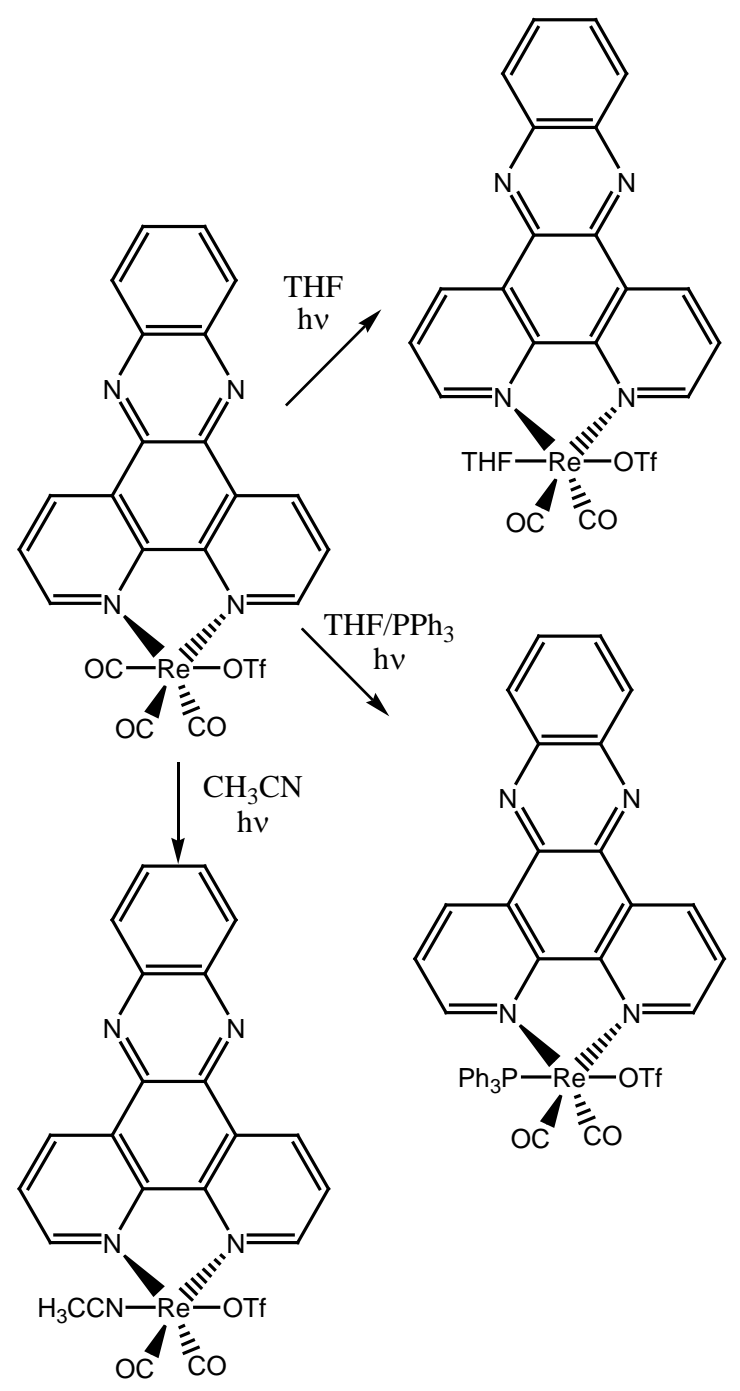

Scheme A1.1 Synthesis of (dppz)Re(L)(CO) ${ }_{2} \mathrm{OSO}_{2} \mathrm{CF}_{3}$

\section{A1.4.2. Fluorescence studies with addition of NO-donor}

The $\mathrm{PPh}_{3}$ and $\mathrm{CH}_{3} \mathrm{CN}$ complexes $\mathbf{A} 2$ and $\mathbf{A} 3$ show no fluorescence spectra. The Re-(dppz)(CO)2(THF)OTf complex A2 showed small fluorescence response to NO addition; UV-Vis spectrum exhibited two signals at $360 \mathrm{~nm}$ and at $380 \mathrm{~nm}$. Figure A1.1 shows the fluorescence spectrum of $\mathbf{A} 2$ at $\lambda_{\text {exc }}=360 \mathrm{~nm}$, upon $\mathrm{NO}$ gradual addition, in the form of NO-donor MAHMA-NONOate $(0-150 \mu \mathrm{M})$ dissolved in $0.04 \mathrm{M} \mathrm{NaOH}$. The emissions at $570 \mathrm{~nm}$ and $424 \mathrm{~nm}$ decrease slightly upon NO addition and the intensity 
changes show linearity with NO-donor concentration (Figure A1.2).

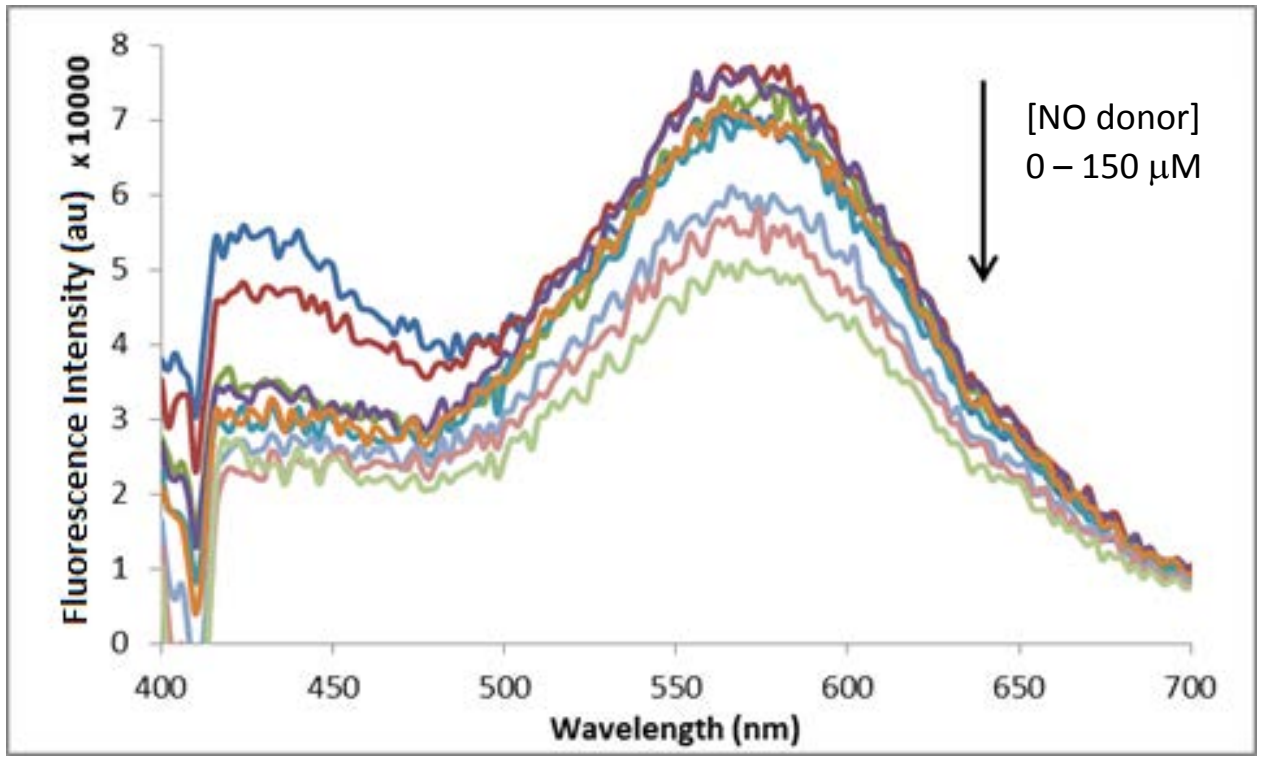

Figure A1.2 Fluorescence Response upon NO-donor addition (0-150 $\mu \mathrm{M})$ to $25 \mu \mathrm{M}$ of Re complex A1 in acetone/PBS aqueous buffer $25 / 75 \mathrm{v} / \mathrm{v},\left(\lambda_{\text {exc }}=360 \mathrm{~nm}\right)$

Figure A1.3 shows the fluorescence spectrum of $\mathbf{A} 2$ at $\lambda_{\mathrm{exc}}=380 \mathrm{~nm}$ upon NO gradual addition, in the form of NO-donor MAHMA-NONOate $(0-150 \mu \mathrm{M})$ dissolved in $0.04 \mathrm{M} \mathrm{NaOH}$. The emission responses were similar to those observed for $\lambda_{\text {exc }}=360$ nm. 


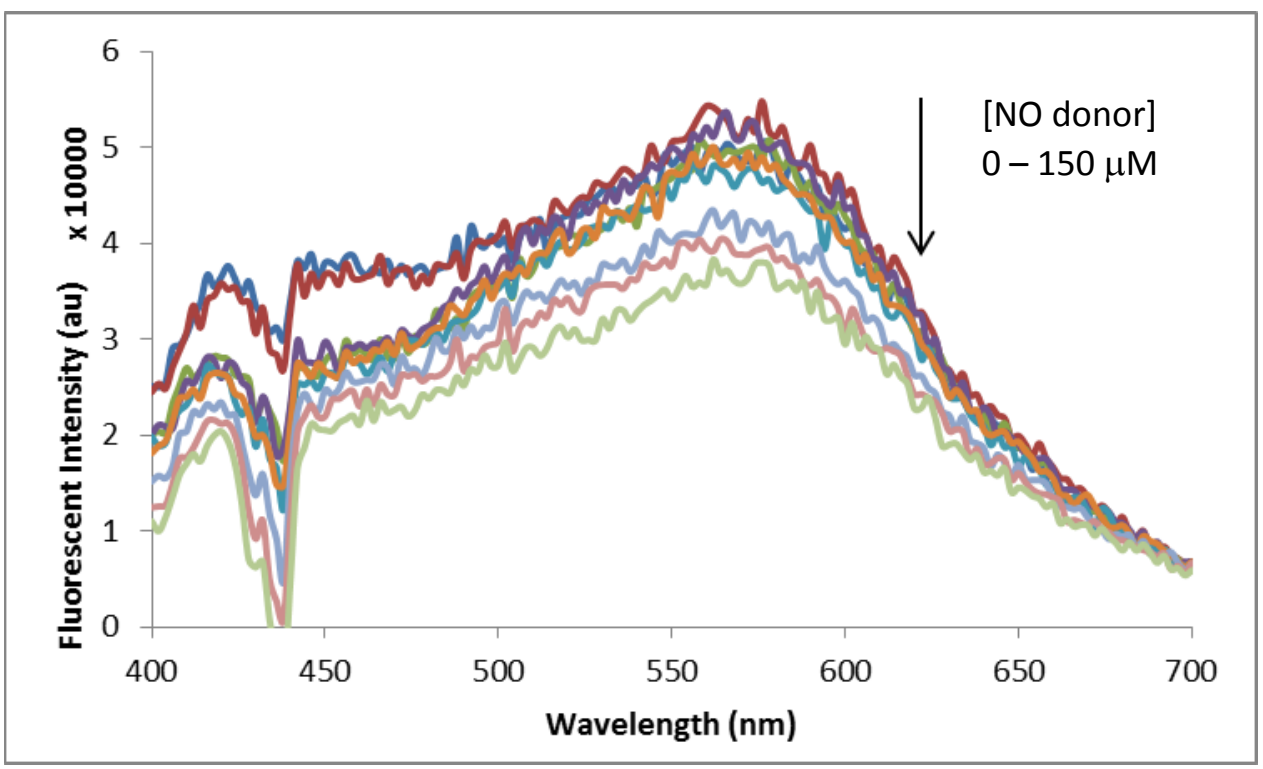

Figure A1.3 Fluorescence Response upon NO-donor addition (0-150 $\mu \mathrm{M})$ to $25 \mu \mathrm{M}$ of Re complex A1 in acetone/PBS aqueous buffer 25/75 v/v, $\left(\lambda_{\text {exc }}=380 \mathrm{~nm}\right)$

\section{A1.4.3. UV-Visible spectroscopy studies with addition of NO-donor}

The THF, $\mathrm{PPh}_{3}$ and $\mathrm{CH}_{3} \mathrm{CN}$ complexes (A2, A3 and A4, respectively) showed significant response upon NO addition under physiological conditions in UV-Vis spectra. Figure A1.4 shows the effects upon NO addition, in the form of NO-donor MAHMANONOate $(0-150 \mu \mathrm{M})$ dissolved in $0.04 \mathrm{M} \mathrm{NaOH}$ to the $\mathrm{CH}_{3} \mathrm{CN}$ complex A4. The absorption at $305 \mathrm{~nm}, 360 \mathrm{~nm}, 380 \mathrm{~nm}$ increased steadily upon NO addition and its intensity showed linear dependence with NO-donor concentration. 


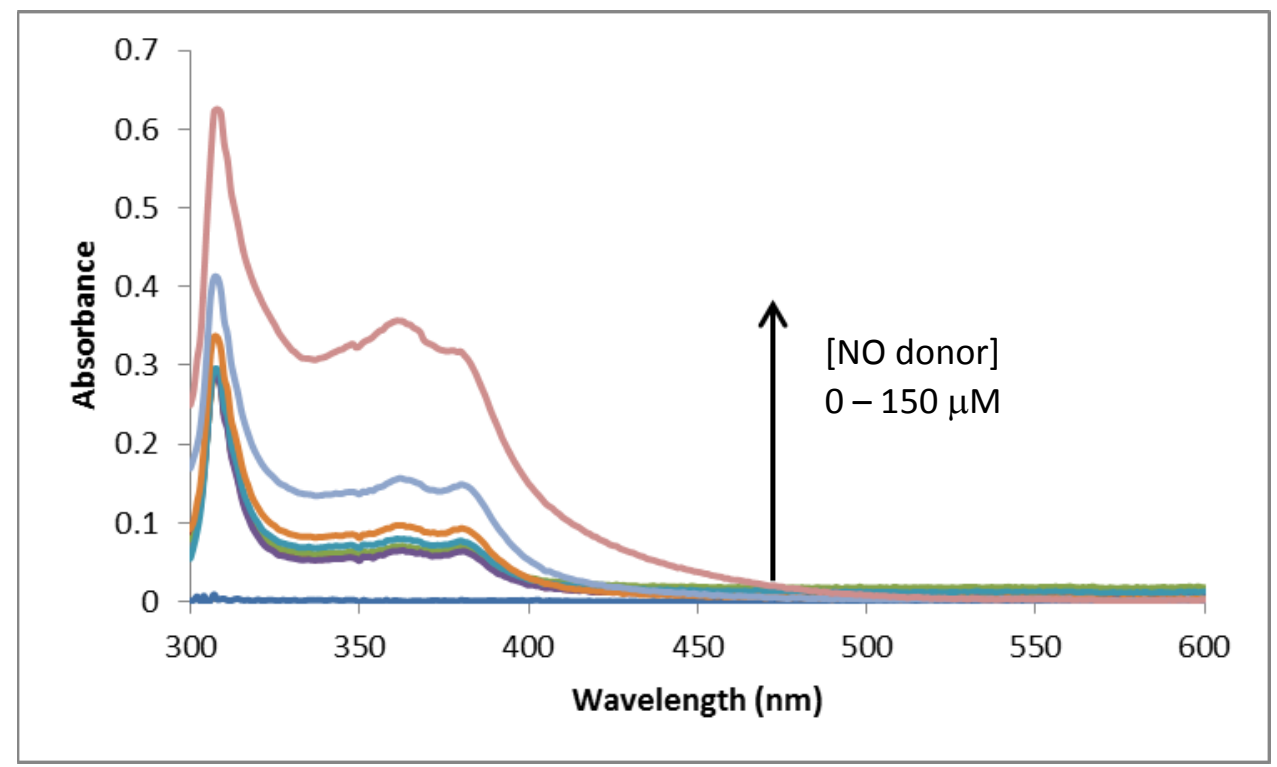

Figure A1.4. UV-Vis Spectra of A4 $(25 \mu \mathrm{M})$ after addition of different amounts of NO donor, in acetone/buffer 25:75.

Figure A1.5 shows the UV-Vis response of A4 with NO concentration. At low concentrations the change is not noticeable. After addition of $100 \mu \mathrm{M} N \mathrm{~N}$, the change in response became more noticeable.

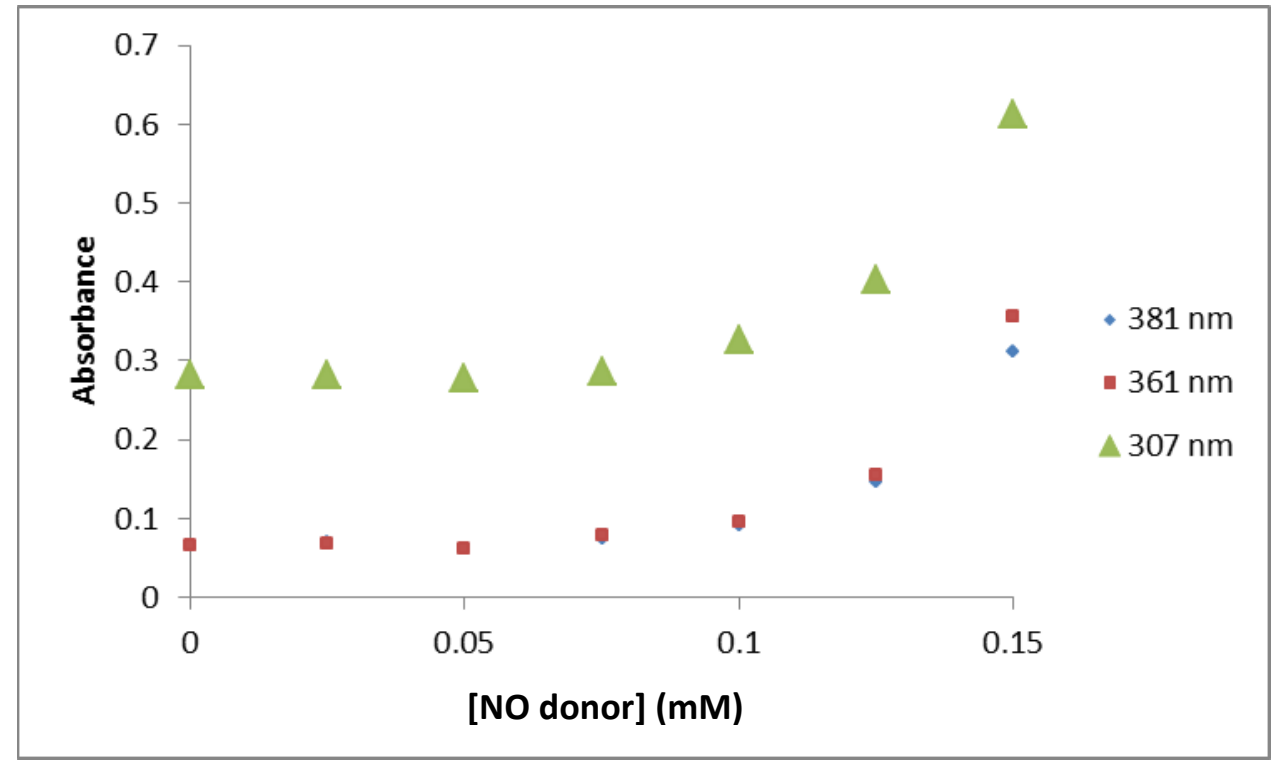

Figure A1.5 UV-Visible absorbance of A4 $25 \mu \mathrm{M} 6$ vs NO concentration, in acetone/buffer 25:75, 
Figures A1.5 and A1.6 show the UV-Vis spectral responses upon NO addition to THF and $\mathrm{PPh}_{3}$ dppz complexes A2 and A3. After $75 \mu \mathrm{mol}$ NO addition, there is a change in the slope of the linear response.

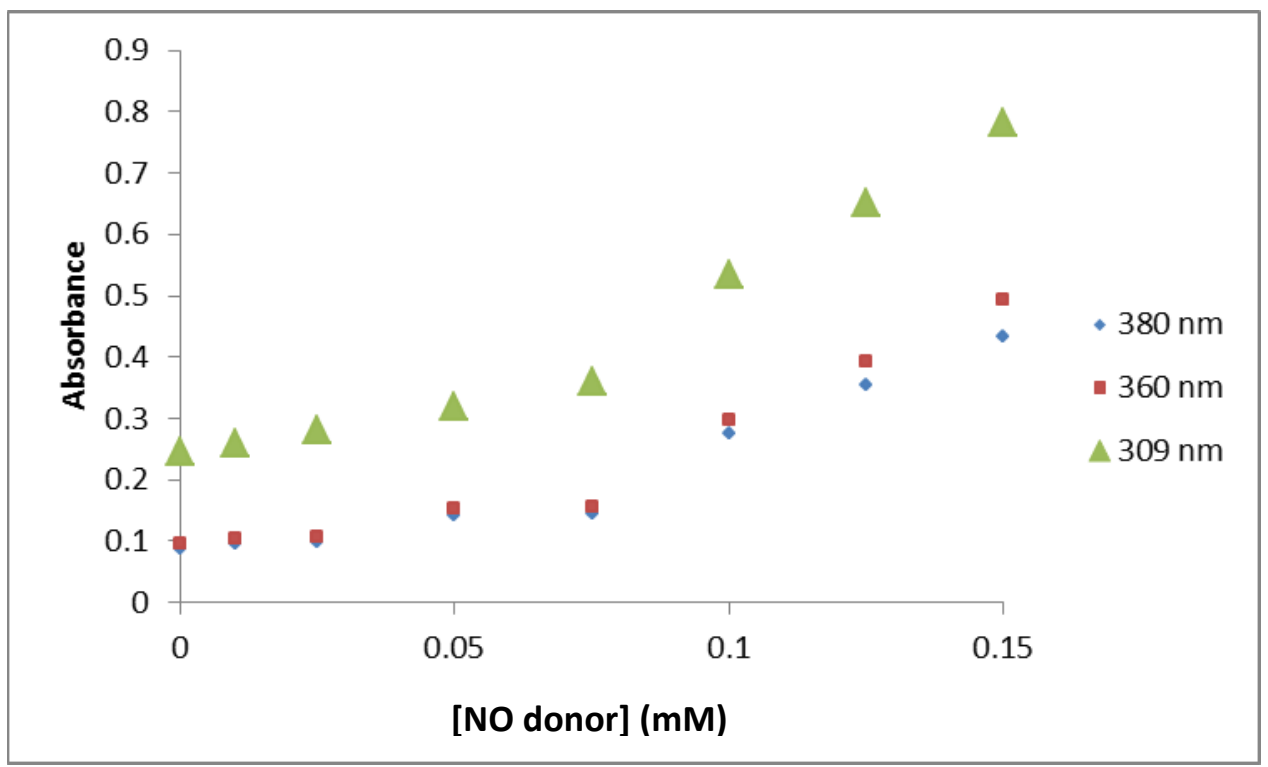

Figure A1.6 UV-Visible absorbance of A2 $25 \mu \mathrm{M} 6$ vs NO concentration, in acetone/buffer 25:75

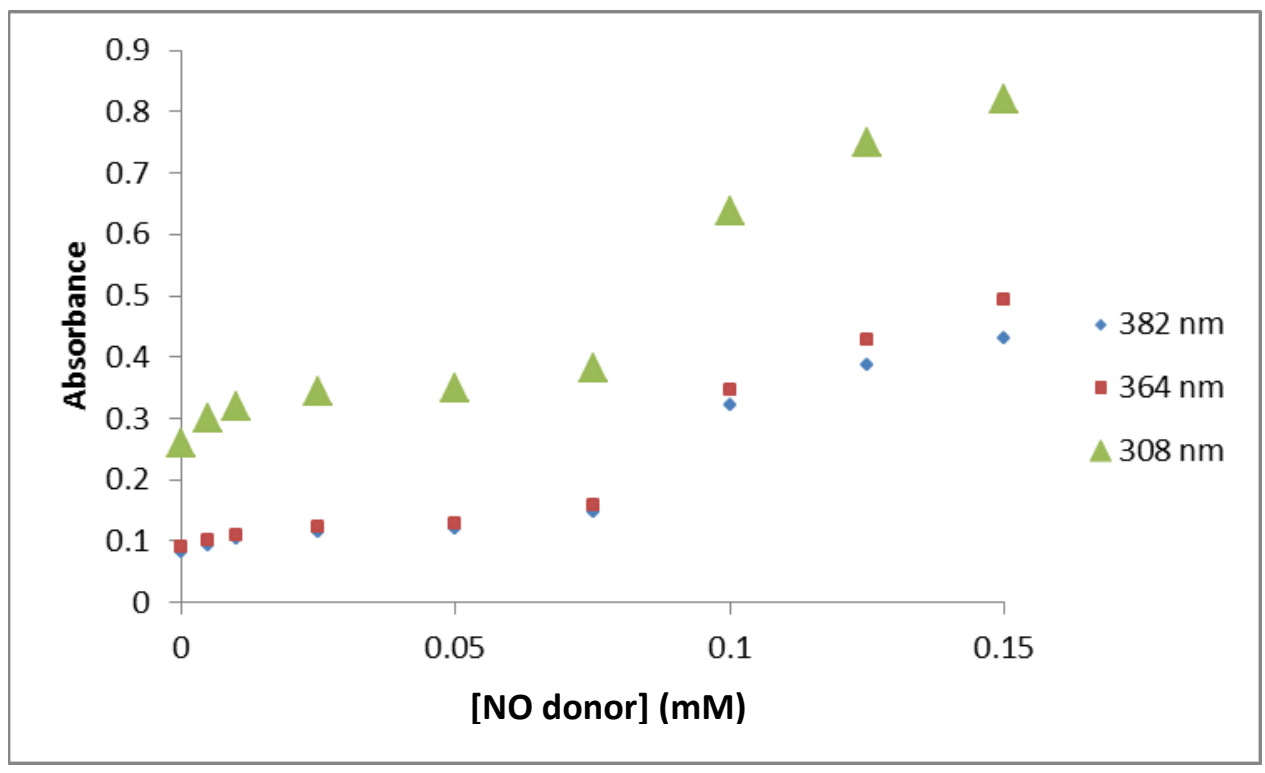

Figure A1.7 UV-Visible absorbance of A3 $25 \mu \mathrm{M} 6$ vs NO concentration, in acetone/buffer 25:75 


\section{A1.4.4. $\quad{ }^{1} \mathrm{H}-\mathrm{NMR}$ and ${ }^{19}$ F-NMR studies on dppz complexes}

Similar to the phenanthroline analogs, ${ }^{1} \mathrm{H}$-NMR showed, for all three NO-active dppz complexes (dppz)Re(L)(CO) 2 OTf (THF for A2, $\mathrm{PPh}_{3}$ for A3, and $\mathrm{CH}_{3} \mathrm{CN}$ A4), that after reaction with NO-donor, the chemical shift of "free - unbound" L - ligand plus a generic “dppz-Re” signals, which are similar for the three dppz-Re complexes. ${ }^{19} \mathrm{~F}-\mathrm{NMR}$ showed that after reaction with NO-donor there is not $\mathrm{CF}_{3} \mathrm{SO}_{3}{ }^{-}$signal (or other $\mathrm{F}$ - containing species). These results help to prove that triflate group $\left(\mathrm{CF}_{3} \mathrm{SO}_{3}{ }^{-}\right)$gets detached from the metal center, and it was discarded in the aqueous layer.

All dppz complexes exhibited only small, yet linear changes in response upon NO addition. Both the initial fluorescence intensities, and the responses of dppz complexes upon NO addition were noteably lower than for the phen complexes. Partial protonation of the basic $\mathrm{N}$ on the dppz framework can explain the lower initial fluorescence intensities in all complexes, because of the dppz ${ }^{3} \mathrm{MLCT}$ is dark; opposite to ${ }^{3} \mathrm{MLCT}$ of phen with is emissive due to environment stabilization. The lower response to NO addition is due to dppz complexes are slightly inert and Re-dppz are less prone to ligand substitution.

\section{A1.5. CONCLUSION}

The (dppz) $\mathrm{Re}(\mathrm{L})(\mathrm{CO})_{2} \mathrm{OSO}_{2} \mathrm{CF}_{3}$ complexes have been synthesized and characterized. These complexes react with NO under physiological conditions, producing a UV-Vis response. The THF complex A2 showed also small quenching in fluorescence, with linear response to NO addition. NMR Studies of all three NO-active complexes proved that after reation with NO triflate and L ligand get disassociated from the metal center. The similarities between phen, DAD, and dppz complexes, toward 
reaction with NO, help to elucidate the possible mechanisms that undergo these $\operatorname{Re}(\mathrm{I})$ complexes.

\section{A1.6. REFERENCES}

1. Kavalllieratos, K; Tsoukias, N. M.; Lozano-Lewis, L. I. "Organometallic dyes for NO detection and imaging". Issued Patent US 8,906,694 B2. Dec. 9, 2014.

2. (a) Choi, C.-S.; Kim, M.-K.; Jeon, K.-S.; Lee, K.-H. J. Lummi. 2004, 109, 121. (b) Choi, C.-S.; Jeon, K.-S.; Lee, K.-H. J. Photosci. 2004, 11, 71. (c) Gestwicki, J. E.; Kiessling, L. L. Nature, 2002, 415, 81. (d) Mason, W. T. Fluorescent and Luminescent Probes for Biological Activity, 2nd ed.; Academic Press: San Diego, 1999. (e) Soper, S. A.; Warner, I. M.; McGown, L. B. Anal. Chem. 1998, 70, 477R. (f) Minta, A.; Kao, J. P. Y.; Tsien, R. Y. J. Biol. Chem. 1989, 264, 8171.

3. Ling, L.-S.; He, Z.-K.; Zhang, P.-Y.; Zeng, Y.-E. J. Nat. Sci. 1999, 4, 482-486.

4. Li, C.; Liu, S.; Guo, L.-H.; Chen, D. Electrochem. Commun., 2005, 7, 23-28.

5. Kalyanasundaram, K. Photochemistry of Polypyridine and Porphyrin Complexes; Academic Press: San Diego, CA, 1992.

6. (a) Turro, C.; Bossmann, S. H.; Jenkins, Y.; Barton, J. K.; Turro, N. J. J. Am. Chem. Soc. 1995, 117, 9026; (b) Friedman, A. E.; Chambron, J. C.; Sauvage, P.; Turro, N. J.; Barton, J. K. J. Am. Chem. Soc. 1990, 112, 4960; FernándezMoreira, V.; Thorp-Greenwood, F. L.; Amoroso, A. J.; Cable, J.; Court, J. B.; Gray, V.; Hayes, A. J.; Jenkins, R. L.; Kariuki, B. M.; Lloyd, D.; Millet, C. O.; Williams, C. Ff.; Coogan, M. P. Org. Biomol. Chem. 2010, 8, 3888.

7. (a) Stoeffler, H. D.; Thornton, N. B.; Temkin, S. L.; Schanze, K. S. J. Am. Chem. Soc. 1995, 117, 7119-7128. (b) Schoonover, J. R.; Bates, W. D.; Meyer, T. J. Inorg. Chem. 1995, 34, 6421-6422. (c) Schoonover, J. R.; Strouse, G. F.; Dyer, R. B.; Bates, W. D.; Chen, P.; Meyer, T. J. Inorg. Chem. 1996, 35, 273-274.

8. Atsumi, M.; Gonzalez, L.; Daniel, C. J. Photochem. Photobiol., A., 2007, 190, 310-320

9. Van der Salm, H.; Fraser, M. G.; Horvath, R.; Cameron, S. A.;. Barnsley, J. E.; Sun, X. - Z.; George, M. W.; Gordon, K. C. Inorg. Chem. 2014, 53, 3126-3140.

10. Wrighton, M., Morse, D .L. J. Am. Chem. Soc., 1974, 96, 998-1003.

11. (dppz) $\operatorname{Re}(\mathrm{CO}){ }_{3} \mathrm{Cl}$ spectroscopic data. FT-IR (cm-1): 2019 (vCO, s), 1884 (vCO, s), 1629, 1603, 1519, 1493, 1428, 1416, 845, 830, 776, 730, 722, 643, 630. ${ }^{1} \mathrm{H}-$ NMR (CDCl 3$): 8.03\left(\mathrm{~d}, 2 \mathrm{H},{ }^{3} \mathrm{~J}_{\mathrm{H}-\mathrm{H}}=8.3 \mathrm{~Hz},{ }^{3} \mathrm{~J}_{\mathrm{H}-\mathrm{H}}=5.2 \mathrm{~Hz}\right), 8.07\left(\mathrm{dd}, 2 \mathrm{H},{ }^{3} \mathrm{~J}_{\mathrm{H}-\mathrm{H}}=\right.$ 
$6.6 \mathrm{~Hz},{ }^{3} \mathrm{~J}_{\mathrm{H}-\mathrm{H}}=3.4 \mathrm{~Hz}$ ), $8.46\left(\mathrm{dd}, 2 \mathrm{H},{ }^{3} \mathrm{~J}_{\mathrm{H}-\mathrm{H}}=6.6 \mathrm{~Hz},{ }^{3} \mathrm{~J}_{\mathrm{H}-\mathrm{H}}=3.5 \mathrm{~Hz}\right.$ ), 9.47 (dd, $\left.2 \mathrm{H},{ }^{3} \mathrm{~J}_{\mathrm{H}-\mathrm{H}}=5.2 \mathrm{~Hz},{ }^{3} \mathrm{~J}_{\mathrm{H}-\mathrm{H}}=1.5 \mathrm{~Hz}\right), 9.87\left(\mathrm{dd}, 2 \mathrm{H},{ }^{3} \mathrm{~J}_{\mathrm{H}-\mathrm{H}}=8.3 \mathrm{~Hz},{ }^{3} \mathrm{~J}_{\mathrm{H}-\mathrm{H}}=1.5 \mathrm{~Hz}\right)$. UV-Vis (in ethanol): $\lambda \max =360 \mathrm{~nm}-\operatorname{br}\left(\varepsilon=1840 \mathrm{~mol}^{-1} \mathrm{~cm}^{-1}\right), 380 \mathrm{~nm}-(\varepsilon=$ $\left.1840 \mathrm{~mol}^{-1} \mathrm{~cm}^{-1}\right)$.

12. (a) Geoffroy, G. L.; Wrighton, M. S. Organometallic Photochemistry; Academic Press: New York, 1979; (b) Lees, A. J. Chem. Rev. 1987, 87, 711-743. 


\section{APPENDIX 2. ESI-MS STUDY OF REACTIVITY OF Re-PHENANTHROLINE COMPLEXES WITH NO}

\section{A2.1. ABSTRACT}

The ESI-MS spectra of the phenanthroline complexes $\operatorname{Re}($ phen $)(\mathrm{CO})_{3} \mathrm{LX}$ $\left(\mathrm{L}=\mathrm{PPh}_{3}, \mathrm{CO}\right.$, THF, $\mathrm{X}=\mathrm{Cl}^{-}, \mathrm{OTf}^{-}$) were collected, after reaction with NO donor. Spectra of $\mathrm{Re}(\mathrm{phen})(\mathrm{CO})_{2} \mathrm{PPh}_{3} \mathrm{OTf}(\mathbf{1})$ and $\mathrm{Re}(\mathrm{phen})(\mathrm{CO})_{2}$ (THF)OTf (2) after reaction with NO showed presence of a peak at $\mathrm{m} / \mathrm{z}=339.4$ (for $\mathbf{1}$ ) and $\mathrm{m} / \mathrm{z}=391.2$ (for 2 ) corresponding to $\left[\operatorname{Re}(\mathrm{CO})_{2}(\mathrm{NO})_{2}\left(\mathrm{H}_{2} \mathrm{O}\right)_{2}\right]^{+}$(for $\left.\mathbf{1}\right)$ and $\left[\operatorname{Re}(\mathrm{CO})_{2}(\mathrm{NO})(\text { acetone })_{2}\right]^{+}$(for 2). The ESI-MS spectra of control reactions of $\mathrm{Re}($ phen $)(\mathrm{CO})_{3} \mathrm{Cl}$ and $\mathrm{Re}(\mathrm{phen})(\mathrm{CO})_{3} \mathrm{OTf}$ with $\mathrm{NO}$, show no peaks of any NO-metal containing species, as expected, since these species do not react with NO (See Chapter 2). Instead those spectra showed a peak at $\mathrm{m} / \mathrm{z}=492.2$, which corresponds to the species $\left[\operatorname{Re}(\text { phen })(\mathrm{CO})\left(\mathrm{H}_{2} \mathrm{O}\right)_{2} \text { (acetone) }\right]^{+}$. This peak was also observed along with other decomposition products for the spectra of $\mathbf{1}$ and $\mathbf{2}$ after NO reaction, yet in lower intensity than the peaks of the NO-containing products.

\section{A2.2. INTRODUCTION}

Due to its versatility, electrospray ionization mass spectrometry (ESI-MS) offers the development for identification of several organic and inorganic compounds. ${ }^{1}$ ESI-MS can detect and characterize single species in complex matrixes, which are not involved in equilibria or solvatation processes. This is very important for the characterization of metal-ligand systems. ESI-MS may indicate the stoichiometry of new complexes. ${ }^{2,3,4}$

The ESI-MS technique uses soft ionization, this characteristic decreases excessive fragmentation and parent compounds in solution can be detected with this technique. Charge and nature of the analyte is determinant for choosing the mode in which can be 
detected more efficiently. This technique offers two modes positive and negative, giving different patterns from the same molecule. ${ }^{5 a}$ Important structural information can be recovered directly from the $\mathrm{m} / \mathrm{z}$; in the case of structural conflicts, MS-MS spectra or isotopic pattern can be used for full identification. ${ }^{5 b}$ ESI-MS has been used to characterize cationic Re(I)-phenanthroline complexes. ${ }^{6}$

\section{A2.3. SAMPLE PREPARATION}

$$
\text { (phen)Re( }\left(\mathrm{PPh}_{3}\right)(\mathrm{CO})_{2} \mathrm{OSO}_{2} \mathrm{CF}_{3} \quad \text { (1), (phen) } \mathrm{Re}(\mathrm{THF})(\mathrm{CO})_{2} \mathrm{OSO}_{2} \mathrm{CF}_{3} \quad \text { (2), }
$$

(phen) $\mathrm{Re}(\mathrm{CO}){ }_{3} \mathrm{Cl}$, or (phen) $\mathrm{Re}(\mathrm{CO})_{3} \mathrm{OSO}_{2} \mathrm{CF}_{3}$ (1 mg of each one) were added to 0.05 $\mathrm{mL}$ of THF in four separate $10 \mathrm{~mL}$ vials. $1.6 \mathrm{~mL}$ of acetone was added. Then $2.4 \mathrm{~mL}$ of PBS aqueous buffer $(\mathrm{pH}=7.2)$ and $0.2 \mathrm{~mL}$ of stock NO-donor solution $5 \mathrm{mM}$ in $0.04 \mathrm{M}$ $\mathrm{NaOH}$ were added sequentially. The mixtures were vortexed for $30 \mathrm{sec}$ and then sonicated for $3 \mathrm{~h}$ at room temperature. Dichloromethane was added (3 x $10 \mathrm{~mL})$, and the organic layer was dried with anhydrous sodium sulfate. In order to remove uncomplexed phenanthroline products, the organic phases were washed with $1 \mathrm{M}$ hydrochloric acid, followed by $1 \mathrm{M}$ sodium bicarbonate, rinsed with deionized water, and dried with anhydrous sodium sulfate. The volatiles were evaporated; the solid residues were redissolved in acetonitrile. The samples were measured using ESI in positive mode. 

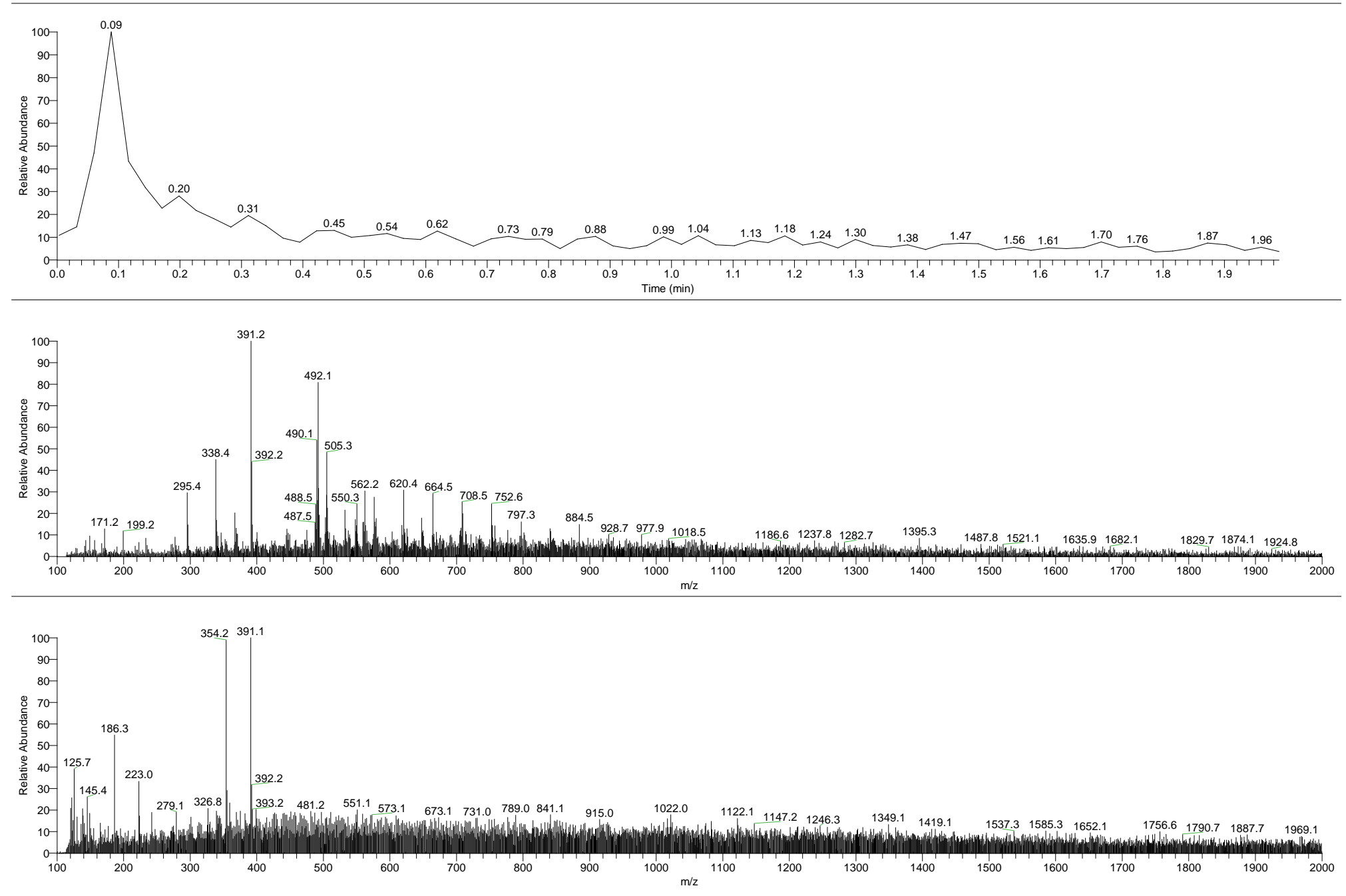

Figure A2.1 ESI-MS positive mode spectrum of (phen)Re(CO)2(THF)OTf 

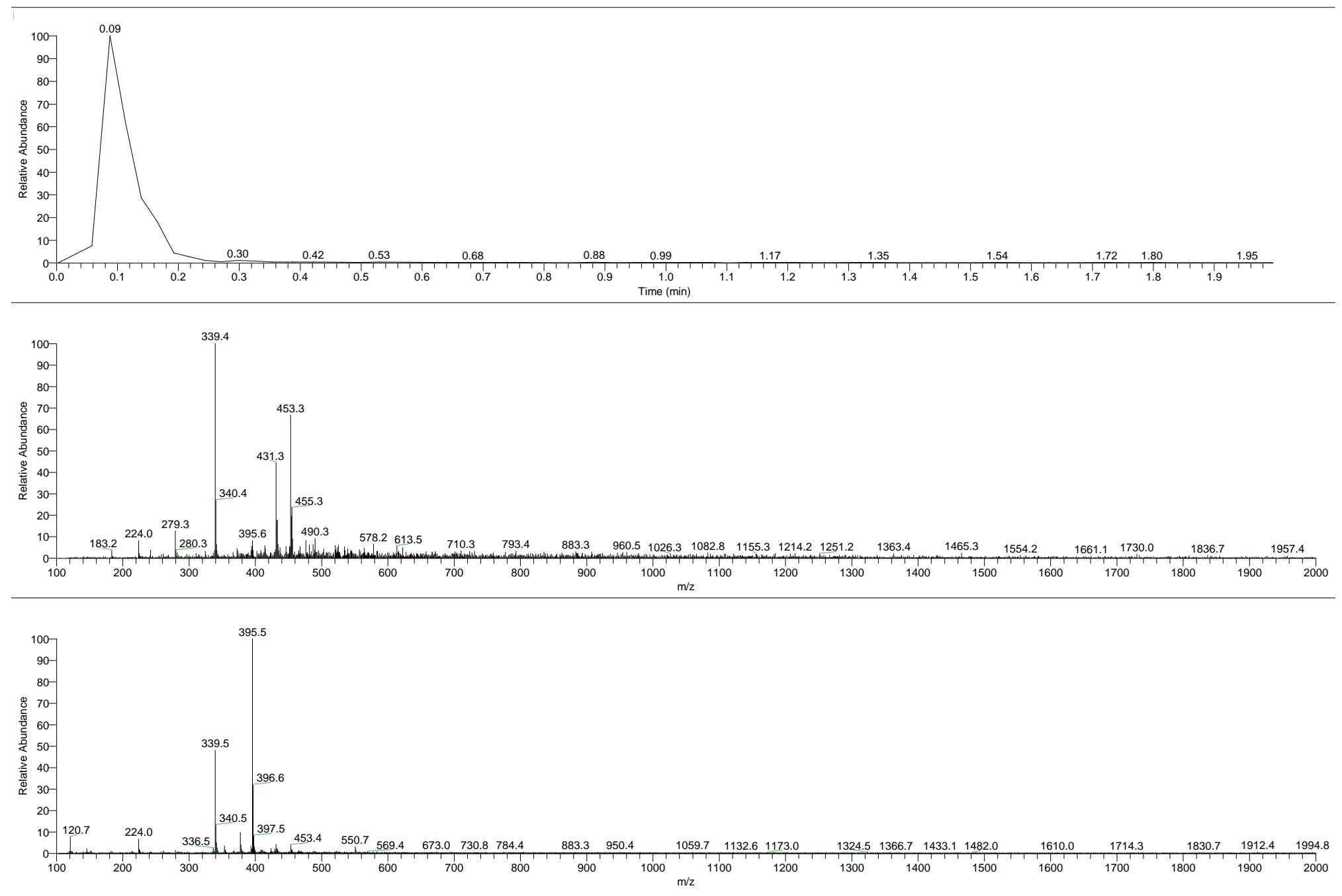

Figure A2.2 ESI-MS positive mode spectrum of (phen)Re(CO) $)_{2}\left(\mathrm{PPh}_{3}\right) \mathrm{OTf}$ 

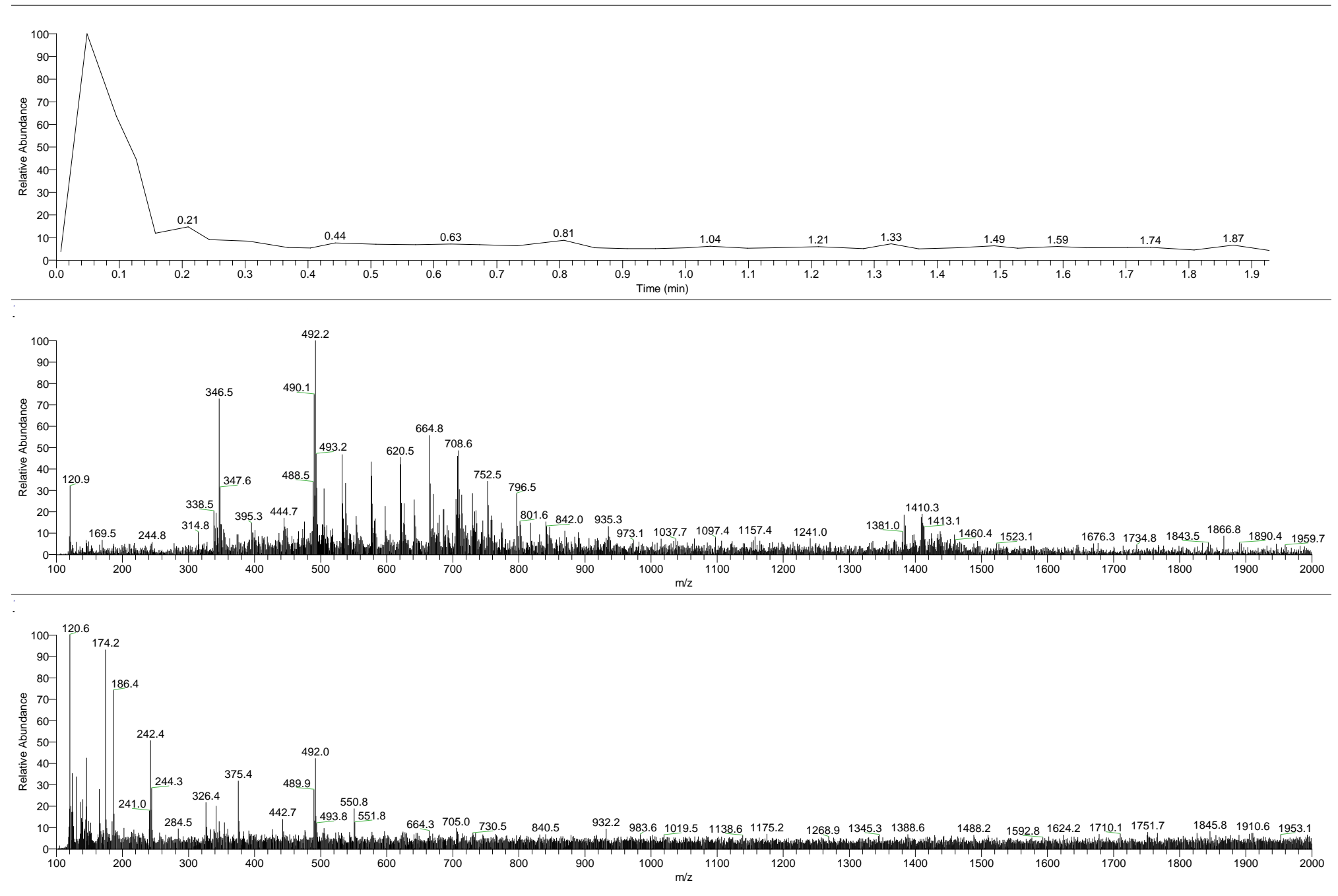

Figure A2.3 ESI-MS positive mode spectrum of (phen) $\operatorname{Re}(\mathrm{CO})_{3} \mathrm{Cl}$ 

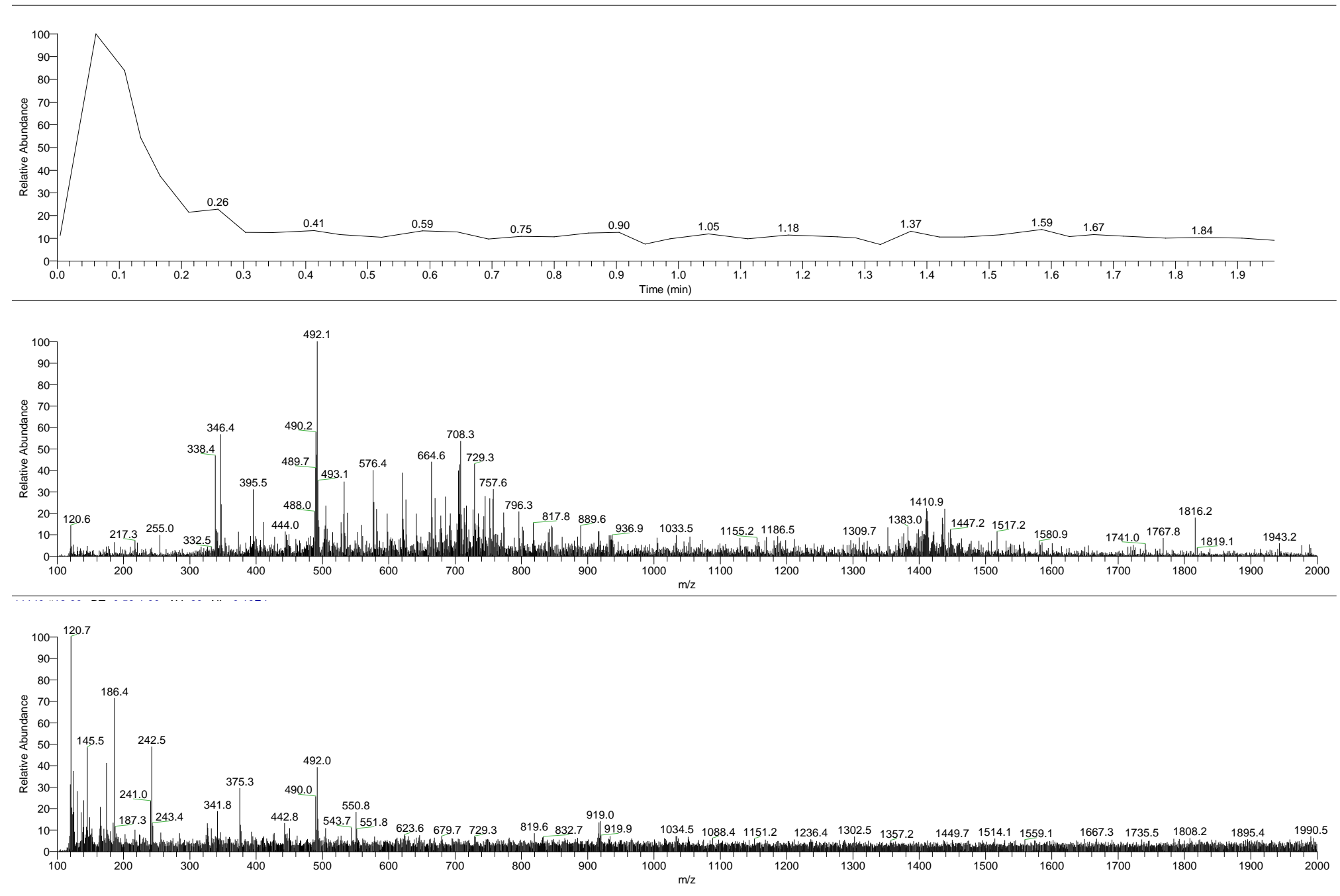

Figure A2.4 ESI-MS positive mode spectrum of (phen)Re(CO) ${ }_{3} \mathrm{OTf}$ 


\section{A2.4. RESULTS AND DISCUSSION}

Different structures can be assigned according to the given $\mathrm{m} / \mathrm{z}$ ratio. The spectra obtained for (phen)Re(THF)(CO) ${ }_{2} \mathrm{OTf}$ (figure A2.1) exhibited a major m/z peak at 391.2 corresponding to $\operatorname{Re}(\mathrm{NO})(\mathrm{CO})_{2}$ (acetone) $)_{2}$, in addition to several solvation products exhibited in Table A2.2.

Table A2.1 List of most important ESI-MS positive mode signals of (phen)Re(THF)(CO) 2 OTf upon reaction with NO-donor.

\begin{tabular}{|c|c|c|c|}
\hline $\mathbf{m} / \mathbf{z}$ ratio & $\begin{array}{c}\text { Relative } \\
\text { abundance }\end{array}$ & Possible Structure \\
\hline 186.3 & 55 & $\mathrm{Re}$ \\
\hline 338.4 & 50 & & \\
\hline 354.2 & 95 & &
\end{tabular}

The spectra obtained for (phen) $\operatorname{Re}\left(\mathrm{PPh}_{3}\right)(\mathrm{CO})_{2} \mathrm{OTf}$ (figure A2.2) exhibited a major m/z peak at 339.4 corresponding to $\mathrm{Re}(\mathrm{NO})_{2}(\mathrm{CO})_{2}\left(\mathrm{H}_{2} \mathrm{O}\right)_{2}$, in addition to several solvation products exhibited in Table A2.3 


\section{Table A2.2}

List of most important ESI-MS positive mode signals of (phen) $\mathrm{Re}\left(\mathrm{PPh}_{3}\right)(\mathrm{CO})_{2} \mathrm{OTf}$ upon reaction with NO-donor.

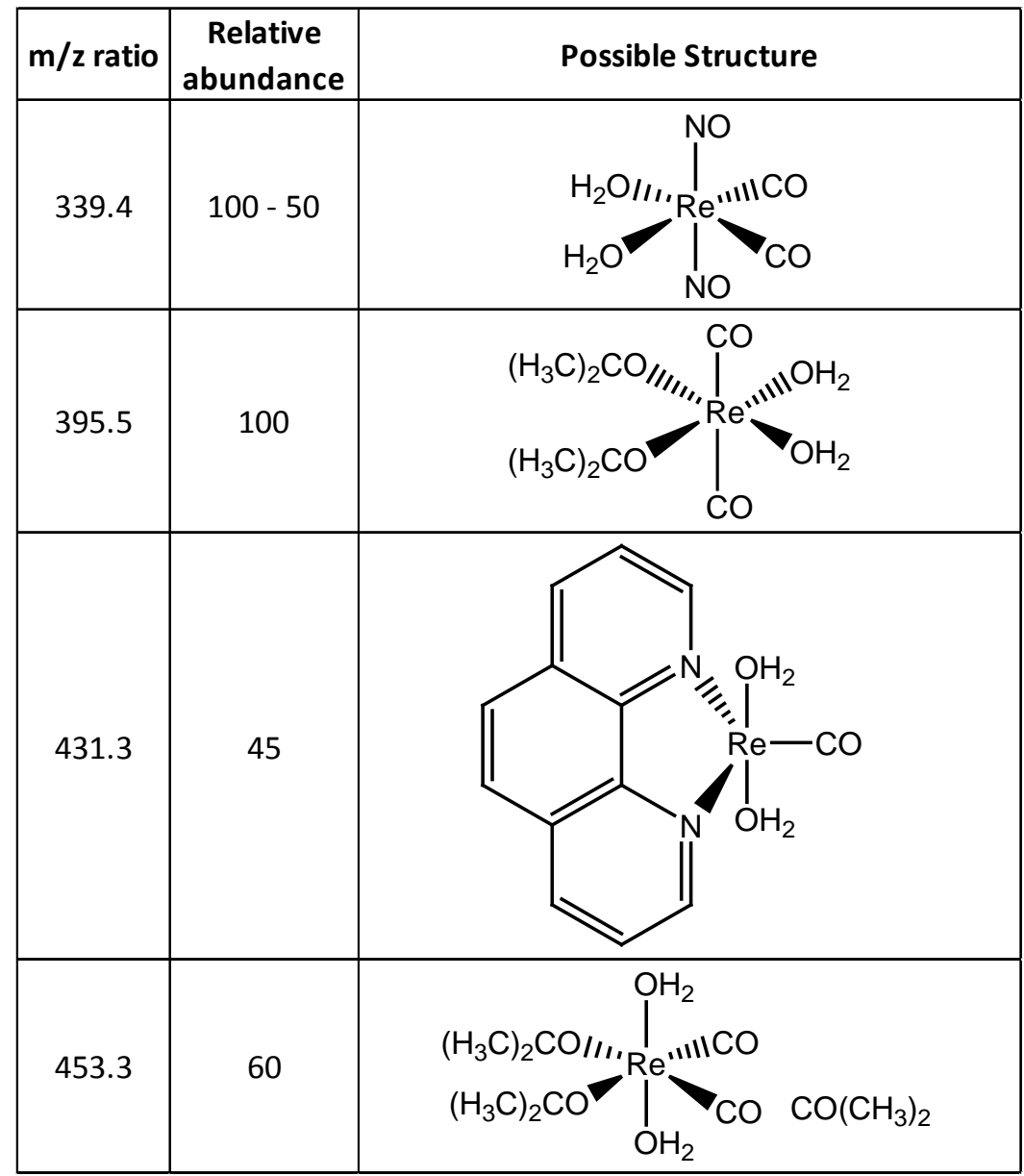

(phen) $\mathrm{Re}(\mathrm{CO})_{3} \mathrm{Cl}$ and (phen) $\mathrm{Re}(\mathrm{CO})_{3} \mathrm{OTf}$ were used as controls. The mass spectra of these complexes showed no Re -NO containing products (Tables A2.3 and A2.4). 
Table A2.3 List of most important ESI-MS positive mode signals of (phen) $\operatorname{Re}(\mathrm{CO})_{3} \mathrm{Cl}$ upon reaction with NO-donor.

\begin{tabular}{|c|c|c|}
\hline $\mathrm{m} / \mathbf{z}$ ratio & $\begin{array}{c}\text { Relative } \\
\text { abundance }\end{array}$ & Possible Structure \\
\hline 186.4 & 72 & $\mathrm{Re}$ \\
\hline 242.4 & 50 & $\mathrm{Re}(\mathrm{OH})_{3}$ \\
\hline 346.5 & 70 & \\
\hline 492.2 & $100-45$ & \\
\hline
\end{tabular}

Table A2.4 List of most prominent ESI-MS positive mode signals of (phen)Re(CO) ${ }_{3} \mathrm{OTf}$ upon reaction with NO-donor.

\begin{tabular}{|c|c|c|}
\hline $\mathrm{m} / \mathbf{z}$ ratio & $\begin{array}{c}\text { Relative } \\
\text { abundance }\end{array}$ & Possible Structure \\
\hline 186.4 & 70 & $\mathrm{Re}$ \\
\hline 242.4 & 50 & $\mathrm{Re}(\mathrm{OH})_{3}$ \\
\hline 338.4 & 48 & \\
\hline 346.4 & 56 & \\
\hline & & \\
\hline
\end{tabular}


The mechanism of action of these phen-Re(I) fluorescent dyes is complex: Two different paths can be identified by spectroscopic techniques: a. Reaction with NO, by elimination of two ligands; b. Deactivation of the initial complex by solvation (substitution with acetone, acetonitrile or water).

\section{A2.5. CONCLUSION}

ESI-MS m/z ratios can be useful to predict possible products after the reaction

with NO-donor. NO-active complexes (phen)Re( $\left(\mathrm{PPh}_{3}\right)(\mathrm{CO})_{2} \mathrm{OTf} \quad(\mathbf{1})$ and (phen)Re(THF)(CO) 2 OTf (2) exhibited $\mathrm{m} / \mathrm{z}$ ratio signals that can be ascribed as NO-Re containing species. Control complexes (phen) $\mathrm{Re}(\mathrm{CO})_{3} \mathrm{OTf}$ and (phen) $\mathrm{Re}(\mathrm{CO})_{3} \mathrm{Cl}$ only exhibited solvation byproduct, without NO in them. Other important fact is that all these formed complexes have net charge of +1 ; charged complexes cannot pass through the phospholipidic bilayer cell membrane.

\section{A2.6. REFERENCES}

1. (a) Yamashita M.; Fenn J. B. J. Phys. Chem., 1984, 88, 4451-4459; (b) J. Phys. Chem. 1984, 88, 4671-4675.

2. Kebarle P.; Tang L. Anal. Chem., 1993, 65, 972A-986A.

3. (a) Colton, R.; Harrison, K. L.; Mah, Y. A.; Traeger, J. C. Inorg. Chim. Acta, 1995, 231, 65-71; (b) Inorg. Chim. Acta, 1995, 231, 87-93.

4. (a) Gatlin, C. L.; Turecek, F. Electrospray Ion Mass Spectrom, 1997, 527-570; (b) Henderson W.; Nicholson B. K.; McCaffrey L. J. Polyhedron, 1998, 17, 42914313.

5. (a) Rosenberg, E. Journal of Chromatography A. 2003, 1000, 841. (b) Di Marco, V. B.; Bombi, G. G. Mass Spectrometry Reviews, 2006, 25, 347- 379

6. Choi, A. W. T.; Poon, C. S.; Liu, H. W.; Cheng, H. K.; Lo, K. New J. Chem., 2013, 37, 1711-1719. 
VITA

\section{LISSETTE I. LOZANO-LEWIS}

Bellavista, Callao, Peru

$1993-1999$

B.S., Chemistry (1999)

Pontificia Universidad Catolica del Peru

San Miguel, Lima, Peru

$1997-2003$

$2003-2004$

$2005-2006$

$2005-2007$

2007-2008

$2009-2010$

$2010-2015$

$2010-2015$

2013

Teaching Assistant

Pontificia Universidad Catolica del Peru

San Miguel, Lima, Peru

Analytical Chemist

DIGESA (General Direction of

Environmental Health)

Lima, Peru

M.Sc., Chemistry

University of North Carolina at Charlotte

Charlotte, North Carolina

Research/Teaching Assistant

University of North Carolina at Charlotte

Charlotte, North Carolina

A\&L Southern Agricultural Laboratories

Pompano Beach, Florida

Research/Teaching Assistant

Florida Atlantic University

Boca Raton, Florida

Doctoral Candidate

Florida International University

Miami, Florida

Research/Teaching Assistant

Florida International University

Miami Florida

First place Graduate Student Poster 15th BCI Symposium.

Miami, Florida 


\section{PUBLICATIONS/PATENTS AND PRESENTATIONS}

Kavalllieratos*, K.; Tsoukias, N. M.; Lozano-Lewis, L. I. "Organometallic dyes for NO detection and imaging". US Patent: 8,906,694 B2. Granted:Dec. 9, 2014 (conversion of provisional application above).

Lozano-Lewis, L. I., John-Rajkumar, S. V., Islas, S. A., Pike, R. D., Rabinovich, D. Bis(thioether)silanes: Synthesis and characterization of tin (IV) complexes with new dithioether ligands. Main group Chemistry. 2007, 6 (3-4), 133-142.

Lozano-Lewis, L. I.; Tsoukias, N. M.; Kavallieratos, K. Metal complexes of phenanthroline and analogs as versatile frameworks for Nitric Oxide sensing and imaging. 243rd ACS National Meeting \& Exposition, San Diego, CA, United States, March 25-29, 2012 (2012), INOR-758 (Poster)

Reynaldo, Y.; Dela Cruz, J. J.; Lozano-Lewis, L. I.; Zhang, Y.; Marder, S. R.; Kavallieratos, K. $\mathrm{Pb}(\mathrm{II})$ extraction and optical sensing by dinitro-phenylenediamine derivatives and phenazine analogs. 243rd ACS National Meeting \& Exposition, San Diego, CA, United States, March 25-29, 2012 (2012), CHED-821 (Poster)

Lozano-Lewis, L. I.; Tsoukias, N. M.; Kavallieratos, K. Metal complexes of phenanthroline and analogs as versatile frameworks for Nitric Oxide sensing and imaging.15th Biomedical and Comparative Immunology Symposium, March 7-8, 2013. Miami, Florida. Awarded best Graduate Poster.

Lozano-Lewis, L. I.; Madrasi, K.; Tsoukias, N. M.; Kavallieratos, K. Re-phenanthroline complexes as ratiometric fluorescent dyes for NO sensing. 245th ACS National Meeting \& Exposition, New Orleans, LA, United States, April 7-11, 2013 (2013), INOR-262 (Poster).

Lozano-Lewis, L. I.; Madrasi, K.; Tsoukias, N. M.; Kavallieratos, K. Ratiometric detection of nitric oxide by fluorescent organometallic Re-phenanthroline complexes. 247th ACS National Meeting \& Exposition, Dallas, TX, United States, March 16-20, 2014 (2014), INOR-866 (Oral).

Venegas, B.; Chiu, Y.; Rodriguez, N.; Lozano-Lewis, L. I.; Kavallieratos, K. Cd(II) optical sensing by a thioamide ligand via ion-exchange extraction. 247th ACS National Meeting \& Exposition, Dallas, TX, United States, March 16-20, 2014 (2014), CHED-768 (Poster). 